\title{
Combining data science and medical imaging
}

Citation for published version (APA):

Coroller, T. P. (2017). Combining data science and medical imaging: Advancing cancer precision medicine with radiomics. [Doctoral Thesis, Maastricht University]. Datawyse / Universitaire Pers Maastricht. https://doi.org/10.26481/dis.20171214tc

Document status and date:

Published: 01/01/2017

DOI:

$10.26481 /$ dis.20171214tc

Document Version:

Publisher's PDF, also known as Version of record

\section{Please check the document version of this publication:}

- A submitted manuscript is the version of the article upon submission and before peer-review. There can be important differences between the submitted version and the official published version of record.

People interested in the research are advised to contact the author for the final version of the publication, or visit the DOI to the publisher's website.

- The final author version and the galley proof are versions of the publication after peer review.

- The final published version features the final layout of the paper including the volume, issue and page numbers.

Link to publication

\footnotetext{
General rights rights.

- You may freely distribute the URL identifying the publication in the public portal. please follow below link for the End User Agreement:

www.umlib.nl/taverne-license

Take down policy

If you believe that this document breaches copyright please contact us at:

repository@maastrichtuniversity.nl

providing details and we will investigate your claim.
}

Copyright and moral rights for the publications made accessible in the public portal are retained by the authors and/or other copyright owners and it is a condition of accessing publications that users recognise and abide by the legal requirements associated with these

- Users may download and print one copy of any publication from the public portal for the purpose of private study or research.

- You may not further distribute the material or use it for any profit-making activity or commercial gain

If the publication is distributed under the terms of Article $25 \mathrm{fa}$ of the Dutch Copyright Act, indicated by the "Taverne" license above, 


\section{Combining Data Science and Medical Imaging:}

Advancing Cancer Precision Medicine with Radiomics

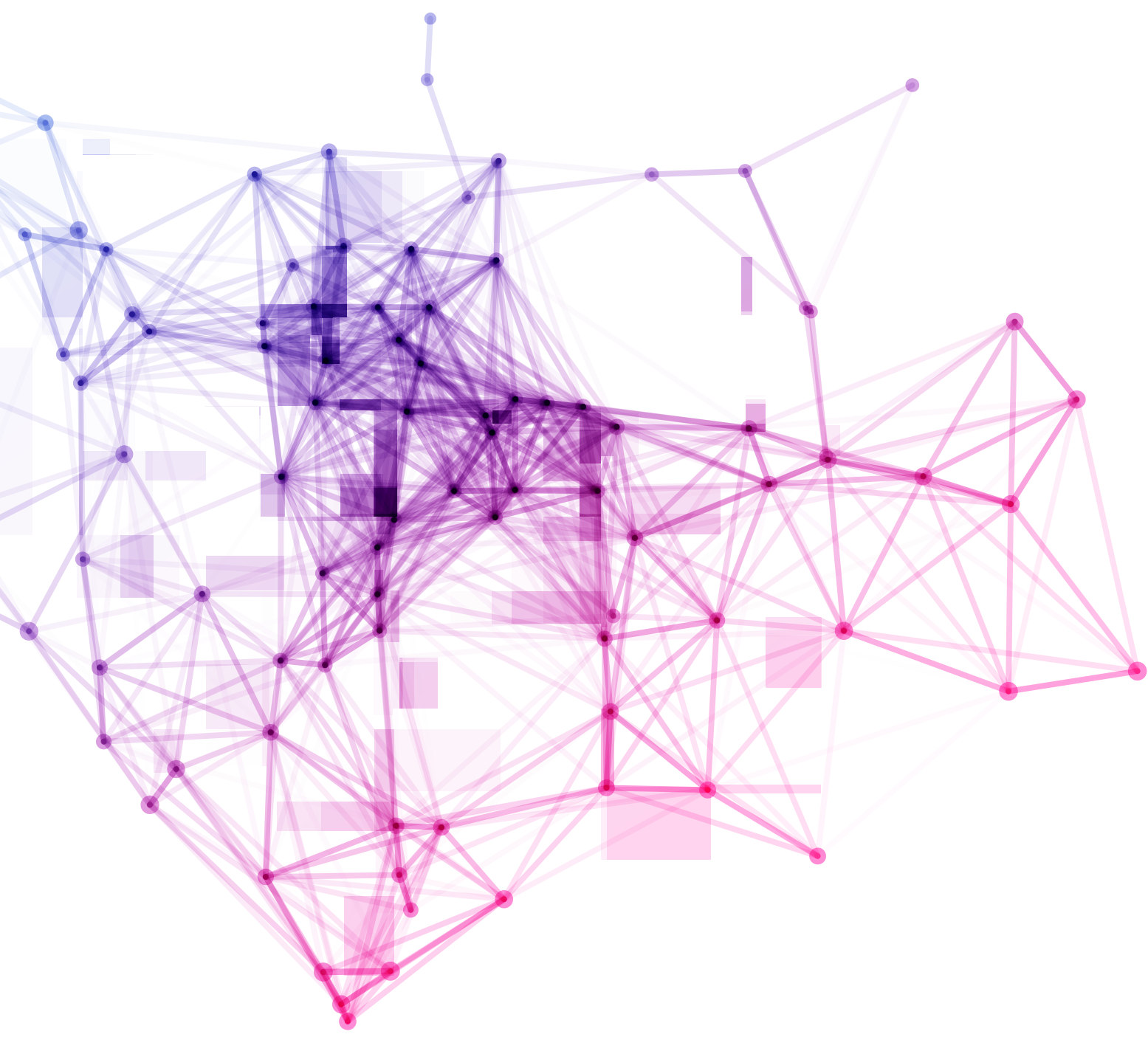

Thibaud Coroller 
(C) copyright Thibaud Coroller, Maastricht 2017

Printing: Datawyse | Universitaire Pers Maastricht

ISBN 9789461597755 


\title{
Combining Data Science and Medical Imaging:
}

\section{Advancing Cancer Precision Medicine with Radiomics}

By

\author{
Thibaud P. Coroller \\ to obtain the degree of doctor \\ at Maastricht University, \\ on the authority of the Rector Magnificus Prof. dr. Rianne M. Letschert, \\ in accordance with the decision of the Board of Deans, \\ to be defended in public on \\ Thursday \\ December $14^{\text {th }}, 2017$ at $10: 00$ hours
}




\section{Promoter}

Prof. Dr. Phillipe Lambin

\section{Co-promoter}

Dr. Ir. Hugo J. W. L. Aerts, Harvard University

\section{Assessment committee}

Prof. Dr. Dirk de Ruysscher, Chairman

Prof. Dr. Johan Bussink, Radbound UMC Nijmegen

Prof. Dr. Andrea Dekker

Prof. Dr. Peeters Ralf

Prof. Dr. Wiero Niessen, Erasmus MC Rotterdam 


\section{Table of Content}

Introduction

Chapter 1 General introduction and outline of the thesis

Part 1 Prediction of Lung Tumor Response to Chemoradiation

Chapter 2 Radiomic phenotype features predict pathological response in Non-Small Cell Lung Cancer

(T Coroller et al. Radiotherapy and Oncology. 2016)

Chapter 3 Radiomic-based Pathological Response Prediction from

Primary Tumours and Lymph Nodes in NSCLC

(T Coroller et al. Journal of Thoracic Oncology. 2017)

Part 2 Lung Tumor Evolution After Radiotherapy Treatment

Chapter 4 CT-based radiomic signature predicts distant metastasis in lung adenocarcinoma

(T Coroller*, P Grossmann* et al. Radiotherapy and Oncology. 2015)

Chapter 5 CT-based radiomic analysis of stereotactic body radiation therapy patients with lung cancer

(E Huynh*, T Coroller* et al. Radiotherapy and Oncology. 2016)

Chapter 6 Associations of radiomic data extracted from static and respiratorygated CT scans with disease recurrence in lung cancer patients treated with SBRT

(E Huynh, T Coroller et al. PLoS ONE. 2017)

Part 3 Tumor Intrinsic Nature Assessment

Chapter 7 Radiographic Prediction of Meningioma Grade by Semantic and

Radiomic features

(T Coroller*, WL Bi* et al. PLoS ONE. 2017)

Chapter 8 Somatic mutations drive distinct imaging phenotypes in lung cancer

(E Rios Velazquez*, C Parmar*, Y Liu*, T Coroller et al. Cancer Research. 2017) 
Chapter 9 Associations between somatic mutations and metabolic imaging phenotypes in non-small cell lung cancer

(SF Yip*, J Kim*, T Coroller et al. Journal of Nuclear Medicine. 2016.)

General Discussion and Future Perspectives

Chapter 10 Conclusion and Perspective

Summary

Contribution Details

Acknowledgement 189

Curriculum Vitae

List of Publications 193

Societal Impact and Valorizations 195 
Introduction 

Chapter

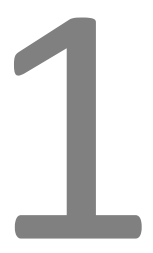

General introduction and outline of the thesis 



\section{INTRODUCTION}

This thesis aims to give insights into the use of radiomics (quantitative imaging features mathematically driven) in cancer management. Radiomics could potentially extract information from existing (and future) medical images and thus become a cost-efficient tool for precision medicine. In this introduction, we will provide an overview of important concepts, then describe the thesis outline.

\section{Medical images}

Medical imaging is the technique and process of creating visual representations of the interior of a body for clinical analysis and medical intervention, as well as visual representation of the function of some organs or tissues. Medical imaging seeks to reveal internal structures hidden by the skin and bones, as well as to diagnose and treat diseases. Medical imaging also establishes a database of normal anatomy and psychology to make it possible to identify abnormalities. Medical image main modalities include computed tomography (CT), magnetic resonance image (MRI) and positron emission tomography (PET). They can be grouped between three major groups:

- Anatomical images: focus on details of the body regardless to their function. Give precise atlas of the organs. Commonly used modalities are CT and MRI.

- Functional images: focus on function of the body (e.g. brain activity). Commonly used modality is MRI.

- Molecular images: focus on a radiotracer that is bonded to specific task (e.g. digestion, tumor metabolism) and quantify a natural process. Commonly used modality is PET.

Each modality has benefits and cons. Therefore, it is not uncommon fusing the information from each type to give at the same time (e.g. PET-CT images). 


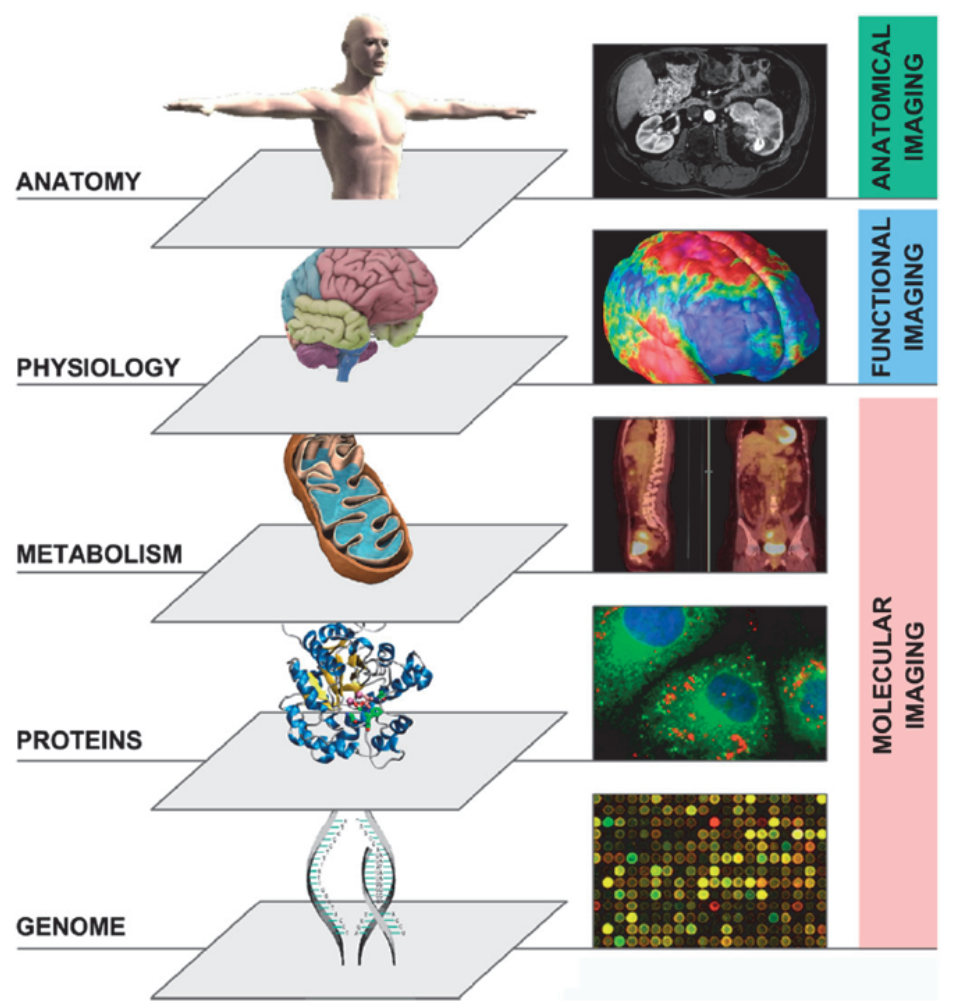

Figure 1. Multilevel imaging: anatomical, functional, and molecular imaging. (Lambin, P. et al. Radiomics: Extracting more information from medical images using advanced feature analysis. European Journal of Cancer 2012)

\section{Cancer}

Cancer is a group of diseases involving abnormal cell growth with the potential to invade or spread to other parts of the body (metastasis). Not all tumors are cancerous; benign tumors do not spread to other parts of the body (e.g. meningioma). Possible signs and symptoms include a lump, abnormal bleeding, prolonged cough, unexplained weight loss and a change in bowel movements. While these symptoms may indicate cancer, they may have other causes. Many cancers can be prevented by healthy habits (no smoking or drinking, maintaining a healthy weight, good diet, vaccinations against certain infectious diseases and avoiding too much sunlight exposure). The chance of survival strongly depends on the type of cancer, the extent of disease at diagnostic and the treatment taken. 


\section{Cancer treatment}

There are many solutions to treat cancer ${ }^{1}$ that could be implemented alone or combined. These include surgery (removing cancer bulk and/or infiltrated area), chemotherapy (chemicals that kill fast-growing cells such as cancer cells) or radiotherapy (high energy beams that destroy genetic material). Each method has its own risks and benefits. Choosing between them will be mostly weighted on clinical information (patient age, pre-existing conditions, and comorbidities) and disease status (tumor stage, prior treatments). A given patient can be given all three treatment types to maximize the chance of response; however, it will likely add risks thus impacts quality of life. Therefore, providing clinicians the ability to gather critical information at an earlier time point could help tailor an efficient treatment for cancer patients that maximizes outcome. As part of the radiation oncology department, our work has been mostly focused on radiotherapy (RT) patients and their outcomes. For lung cancer RT, patients often undergo several weeks of treatment with a daily dose of a couple grays (ionozing dose units). On the other hand, stereotactic radiosurgery (SRS) is a high-dose hypo-fractionated scheme, where the patient will have very limited days of treatment (only 3 to 5 ) with much higher dose to the tumor (e.g 18 grays). This option is used for limited tumor stages and for patient with comorbidities that prevent them from having surgery (such as advanced age or diabetes).

\section{Precision medicine}

Until now, most medical treatments have been designed for the "average patient" and should fit most of the people. As a result of this "one-size-fits-all" approach, treatments can be very successful for some patients but not for others. Precision medicine ${ }^{2-4}$, on the other hand, is an innovative approach that takes into account individual differences in genes, environments, and lifestyles (Figure 1). This approach can also be extended to the disease itself. In the case of solid tumor cancer, the tumor phenotype (traits) could give a lot of information about its nature and evolution. Precision medicine gives medical professionals the resources they need to tailor specific treatments of the illnesses we encounter, further develops our scientific and medical research, and keeps our patients healthier.

With over 1.6 million new cases and 500,000 deaths in 2016, cancer is the most common type of disease across genders in the United States ${ }^{5}$. Better predictors of prognosis that can guide more accurate decision-making in treatment could have tremendous impact on many patients as well as the healthcare system. Better treatment decisions will help reduce physical, emotional, and financial burden on patients; and on a larger scale, it will reduce the overall cost on the healthcare system through avoiding unnecessary surgeries, complications, and medications. With the overall cost for cancer care 
in the US estimated at $\$ 87.7$ billion (2014 figure) $)^{6}$, and lung cancer being a significant contributor to the burden, any improvement in treatment and outcome could help reduce healthcare cost significantly. Against this backdrop, radiomics ${ }^{7}$ is an important innovative new field that leverages collaborations across many areas to advance our knowledge in cancer prevention, treatment, and cure.

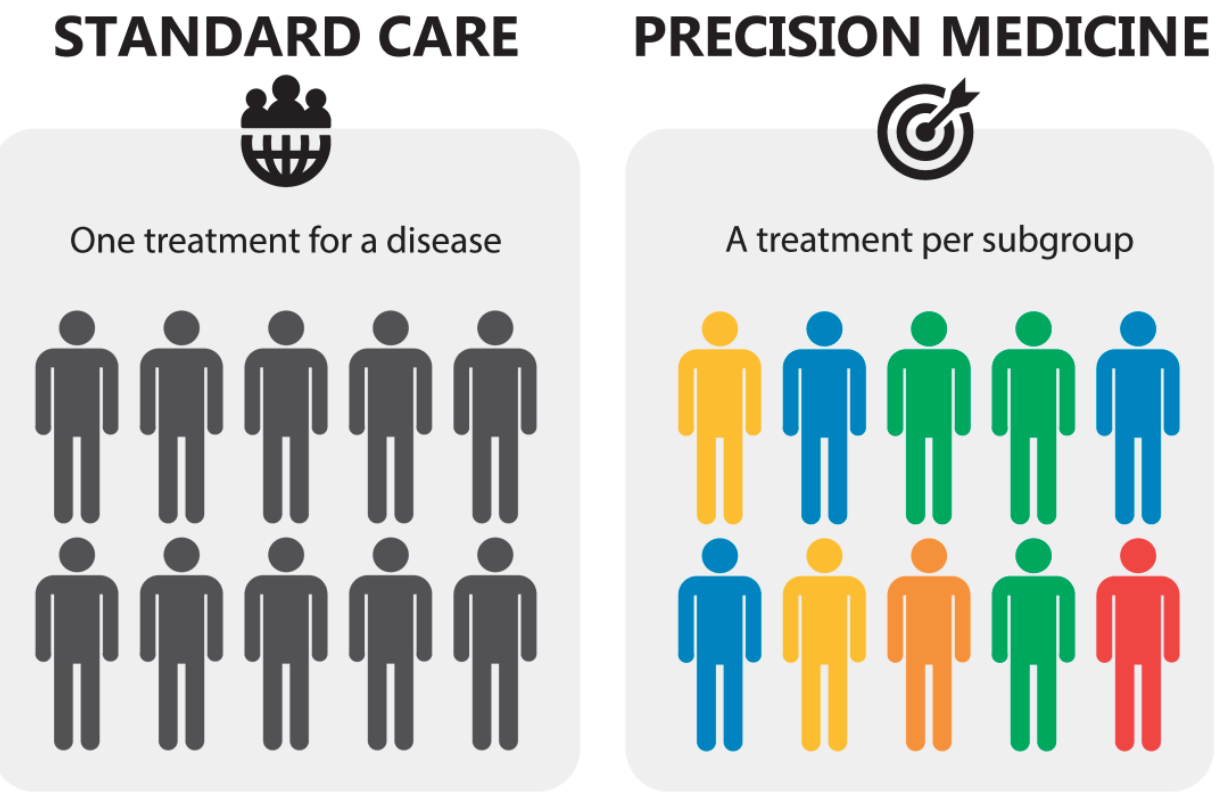

Figure 2. Illustration of standard versus precision medicine. In a standard approach, a group of patients for a given disease will go under a similar treatment (e.g. surgery). In the case of precision medicine, subgroups of patients can be created based on other information such as radiomics. Thus, each subgroup will undergo tailored treatment to optimized its effect.

\section{Radiomics}

The number of medical images and the ability to store them is increasing over the years. New technologies have enabled immense storage and powerful computational cloud that securely connect institutions and scientists from across the world. In the near future, these advances will accelerate our progress in precision medicine by giving access to research to larger number of resources. Radiomics ${ }^{7-10}$, an emerging field where many others intersect- medical physics, biostatistics, and computational biology, finds its significant application in recent cancer research. Radiomics can extract large amount of data from medical images, uncovering advanced features that characterize tumours non-invasively through data analysis. Those features can be extracted from any image type such as computed tomography (CT), positron emission tomography (PET), or mag- 
netic resonance imaging $(\mathrm{MRI})^{11}$. These features can robustly create a unique phenotypic atlas for each tumor (Figure 2).

Associating clinical information to this atlas has enabled the identification of new, reproducible, image-based biomarkers, which have been used to predict tumor response to a specific treatment and understand tumor evolution or its intrinsic biology. Such tools will enable precision medicine in cancer treatment at an earlier time, providing crucial information to guide clinician decisions.

\section{RADIOMICS}

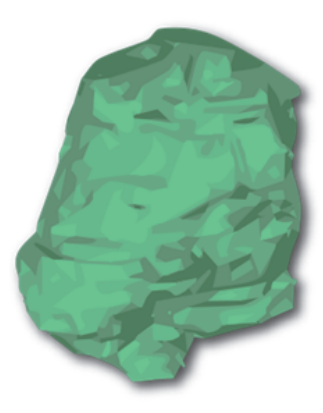

SHAPE FEATURES
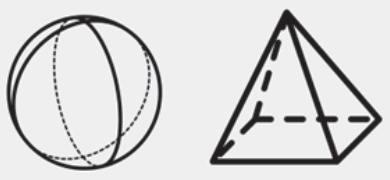

STATISTICS FEATURES
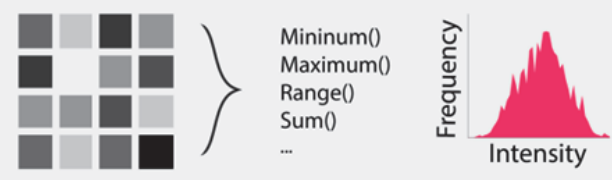

TEXTURAL FEATURES
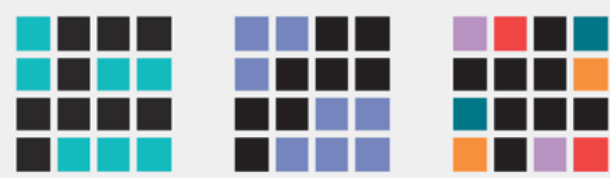

Figure 3. Description of radiomic features that can be extracted from solid tumors. Radiomics commonly include: morphologic features: measure shape and volume of the tumor; Statistics features: quantify simple information of image and Textural features: sensitive to complex pattern in the images, 2D/3D motif of voxel intensity

\section{THESIS OBJECTIVE}

This thesis investigates three main areas where radiomics can be implemented in the clinic:

I. To quantify the likelihood of lung cancer tumor's response to treatment (no response or complete response).

II. To identify lung cancer patient at risk of disease evolution (e.g. distant metastasis, local recurrence) post treatment 
III. To highlight the link between tumor's intrinsic nature and its phenotype, therefore exploring the potential use of quantitative imaging as non-invasive biopsy.

\section{THESIS OUTLINE}

The work of this thesis is presented in three parts, highlighting crucial steps where radiomics can improve clinical practices. Each step is synthetized in Figure 3 and described below.

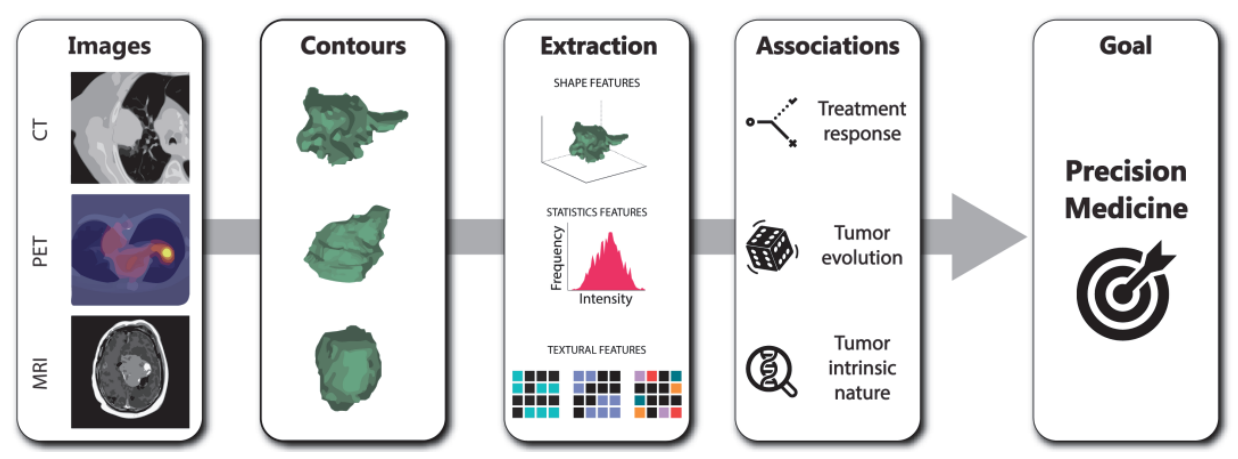

Figure 4. Introduction figure to the concept of radiomics and its potential use in precision medicine through creating a minable database from pre-treatment images (courtesy of Dr. Elizabeth Huynh).

To begin, in Chapter 1, a general introduction of the work presented in this thesis is given. We also introduce the concept of precision medicine and radiomics.

\section{PART 1: PREDICTION OF LUNG TUMOR RESPONSE TO CHEMORADIATION}

This part of the thesis describes the potential of extracting advanced quantitative features from medical images for tumor response prediction. In this section, we ask whether there is more information in medical imaging than what is currently used (currently only volume and diameter as reported). Specifically, we proposed a methodology for high-throughput extraction of quantitative imaging parameters from pre-treatment planning CT for patients treated with trimodality (chemoradiation followed by surgery).

In Chapter 2, in a retrospective study of 127 NSCLC patients treated with trimodality, we investigated the link between tumour traits and its response to treatment ${ }^{12}$. Using pathological confirmation from the surgery as the ground truth, allowing us to classify patients into complete or non-responders. 
We enhanced the investigation in Chapter 3 , by adding radiomics information extracted from involved lymph nodes with 85 patients from our institution ${ }^{13}$. The purpose of this study was to compare information from two critical disease sites in term of response prediction.

\section{PART 2: LUNG TUMOR EVOLUTION AFTER RADIOTHERAPY TREATMENT}

This second part of the thesis focuses on lung tumor evolution and outcomes prediction. We investigated two homogenous first line radiotherapy cohorts, one conventional radiotherapy (RT) and one stereotactic (SBRT), all treated at our institution.

In Chapter 4, we are building a radiomics signature to predict distant metastasis (DM) and overall survival (OS) in a cohort of 182 patients treated with $\mathrm{RT}^{14}$. A patient that will develop DM would have a much lower survival compared to a patient that does not. Therefore, distinguishing prior treatment of such patients could allow doctors to have a closer follow-up and/or add chemotherapy along with RT.

In Chapter 5, we investigated the prognosis (DM and/or local recurrence) of patient treated with SBRT using free breathing (FB) images from 105 patients ${ }^{15}$.

We elevated the analysis in Chapter 6, comparing radiomics extracted from FB versus average intensity projection (AIP) images in 90 patients treated with SBRT ${ }^{16}$. The purpose was to assess whether more information was available from "snapshot" images (FB) versus long exposure pose (AIP).

\section{PART 3: TUMOR INTRINSIC NATURE ASSESSMENT}

In the last part of the thesis, we investigate on the biological association between tumour phenotype and genetic information. The hypothesis is that a given tumor with its genetic burden (e.g. somatic mutation or tumor grade) would present unique traits and characteristics. Therefore, we could use medical images to replace or complement biopsy. This could be valuable especially for location where biopsy is risky due to motion (lung) or critical tissue (brain).

In Chapter 7, we found a radiographic prediction tool for meningioma tumor aggressiveness (high grade) prior to surgery using 175 T1 post contract MRI images of meningioma patients that underwent surgery and had pathological assessment.

Chapter 8 quantifies the link between somatic mutations (EGFR and KRAS) and radiomic features extracted from 763 lung adenocarcinoma cases with CT images from four different cohorts. This large scale and multicentre study gave additional insight of the link between phenotype and genome. 
Chapter 9 also investigates somatic mutations but with radiomic features extracted from positron emission tomography (PET) images in 348 lung cancer patients ${ }^{17}$.

\section{GENERAL DISCUSSION AND FUTURE PERSPECTIVES}

Chapter 10 provides a general discussion of the results presented in this thesis and its future outlook and perspectives. 


\section{REFERENCES}

1. Cancer Treatments Description. National Cancer Institute Available at: https://www.cancer.gov/aboutcancer/treatment.

2. The Precision Medicine Initiative. The White House Available at: https://obamawhitehouse.archives.gov/ precision-medicine.

3. Collins, F. S. \& Varmus, H. A new initiative on precision medicine. N. Engl. J. Med. 372, 793-795 (2015).

4. Lambin, P. et al. Predicting outcomes in radiation oncology--multifactorial decision support systems. Nat. Rev. Clin. Oncol. 10, 27-40 (2013).

5. Siegel, R. L., Miller, K. D. \& Jemal, A. Cancer statistics, 2016: Cancer Statistics, 2016. CA. Cancer J. Clin. 66, 7-30 (2016).

6. American Cancer Society. Economic Impact of Cancer. Available at: https://www.cancer.org/cancer/ cancer-basics/economic-impact-of-cancer.html.

7. Lambin, P. et al. Radiomics: Extracting more information from medical images using advanced feature analysis. Eur. J. Cancer 48, 441-446 (2012).

8. Aerts, H. J. W. L. et al. Decoding tumour phenotype by noninvasive imaging using a quantitative radiomics approach. Nat. Commun. 5, (2014).

9. Gillies, R. S. et al. Metabolic response at repeat PET/CT predicts pathological response to neoadjuvant chemotherapy in oesophageal cancer. Eur. Radiol. 22, 2035-2043 (2012).

10. Hatt, M., Tixier, F., Visvikis, D. \& Cheze Le Rest, C. Radiomics in PET/CT: more than meets the eye? J. Nucl. Med. (2016). doi:10.2967/jnumed.116.184655

11. Aerts, H. J. W. L. The Potential of Radiomic-Based Phenotyping in Precision Medicine: A Review. JAMA Oncol. (2016). doi:10.1001/jamaoncol.2016.2631

12. Coroller, T. P. et al. Radiomic phenotype features predict pathological response in non-small cell lung cancer. Radiother. Oncol. (2016). doi:10.1016/j.radonc.2016.04.004

13. Coroller, T. P. et al. Radiomic-Based Pathological Response Prediction from Primary Tumors and Lymph Nodes in NSCLC. J. Thorac. Oncol. 12, 467-476 (2017).

14. Coroller, T. P. et al. CT-based radiomic signature predicts distant metastasis in lung adenocarcinoma. Radiother. Oncol. 114, 345-350 (2015).

15. Huynh, E. et al. CT-based radiomic analysis of stereotactic body radiation therapy patients with lung cancer. Radiother. Oncol. 120, 258-266 (2016).

16. Huynh, E. et al. Associations of Radiomic Data Extracted from Static and Respiratory-Gated CT Scans with Disease Recurrence in Lung Cancer Patients Treated with SBRT. PloS One 12, e0169172 (2017).

17. Yip, S. S. et al. Associations between somatic mutations and metabolic imaging phenotypes in non-small cell lung cancer. J. Nucl. Med. (2016). doi:10.2967/jnumed.116.181826 



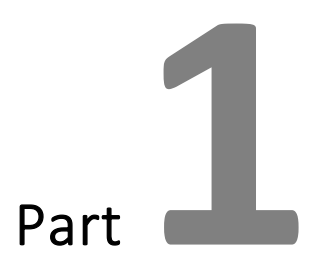

\section{Prediction of Lung Tumor Response to \\ Chemoradiation}





\section{Chapter}

\section{Radiomic phenotype features predict pathological response in Non-Small Cell Lung Cancer}

Published in: Radiotherapy and Oncology (2016)

Radiomic phenotype features predict pathological response in Non-Small Cell Lung Cancer Thibaud Coroller, Vishesh Agrawal, Vivek Narayan, Ying Hou, Patrick Grossmann, Stephanie Lee, Raymond Mak*, Hugo Aerts* 


\section{ABSTRACT}

Background and purpose: Radiomics can quantify tumor phenotype characteristics noninvasively by applying advanced imaging feature algorithms. In this study we assessed if pre-treatment radiomics data are able to predict pathological response after neoadjuvant chemoradiation in patients with locally advanced non-small cell lung cancer (NSCLC).

Material and Method: 127 NSCLC patients were included in this study. Fifteen radiomic features selected based on stability and variance were evaluated for its power to predict pathological response. Predictive power was evaluated using area under the curve $(A \cup C)$. Conventional imaging features (tumor volume and diameter) were used for comparison.

Results: Seven features were predictive for pathologic gross residual disease (AUC $>0.6$, $p$-value $<0.05$ ), and one for pathologic complete response ( $A \cup C=0.63, p$-value $<0.05$ ). No conventional imaging features were predictive (range $A \cup C=0.51$ to 0.59 , $p$-value>0.05). Tumors that did not respond well to neoadjuvant chemoradiation were more likely to present rounder shape (spherical disproportionality, $A \cup C=0.63, p$-value $<0.05$ ) and heterogeneous texture (LoG 5 mm 3D - GLCM entropy, $A U C=0.61$, p-value $<0.05$ ).

Conclusion: We identified predictive radiomic features for pathological response, although no conventional features were significantly predictive. This study demonstrates that radiomics can provide valuable clinical information, and performed better than conventional imaging features. 


\section{INTRODUCTION}

Radiomics is an emerging field of quantitative imaging that aims to describe tumors non-invasively using a large set of advanced imaging features ${ }^{1-3}$. These features can robustly create a unique phenotypic atlas for each tumor ${ }^{4-6}$. Associating clinical information to this atlas has enabled the identification of new, reproducible, image-based biomarkers which has been prognostic for clinical outcomes including overall survival ${ }^{7-9}$ and distant metastasis ${ }^{10}$. Association were found with lung cancer patients of histology and stage ${ }^{11}$ as well.

Lung cancer is the leading cause of cancer deaths worldwide ${ }^{12}$. Stage IIIA non-small cell lung cancer (NSCLC) can be treated using trimodality therapy that includes neoadjuvant chemoradiation followed by surgery according to NCCN guidelines ${ }^{13}$. However, trimodality therapy is controversial, given the observed lack of survival benefit in adding surgery compared to definitive chemoradiation alone, ${ }^{14,15}$ which underscores the importance of identifying patients who respond completely to chemoradiation and do not require additional invasive local therapy.

Pathological response is a direct measure of tumor response to neoadjuvant chemoradiation assessed at time of surgery. It has the potential to be used as a surrogate endpoint ${ }^{16}$ for survival/local control and has been shown to be prognostic for survival in early ${ }^{17}$ and advanced ${ }^{18}$ stages for NSCLC patients. Predicting pathological response at an early time point would allow modification of the treatment regimen (e.g. adding surgery versus intensifying chemoradiation) based on how the tumor is likely to respond and this adaptive approach could improve patient outcomes.

Currently, tumor response is clinically assessed using RECIST ${ }^{19}$, which classifies changes in the sum of tumor and lymph nodes diameters on CT images before and after therapy. However, the radiographic response to chemoradiation for NSCLC tumors may be slow 20 , which may limit the utility of RECIST in predicting pathological response at the end of the neoadjuvant chemoradiation shortly before surgery, and hence allow very little margins for clinicians to adapt treatment regimen consequently.

In this study we investigated the power of pre-treatment CT-based radiomic features to predict pathological response after neoadjuvant chemoradiation. We compared these results to conventional volumetric features such as tumor volume and diameter. 


\section{METHOD}

\section{Patient selection}

Patients with stage II-III NSCLC treated at Dana-Farber Cancer Institute between 2001 and 2013 who were treated with neoadjuvant radiotherapy and chemotherapy (chemoradiation) prior to surgical resection were included in this study. Patients with distant metastasis at presentation or delay in surgery greater than 120 days after the completion of chemoradiation were excluded. For all patients, CT imaging at the initiation of chemoradiation and prior to surgical resection were available. No exclusion based on histology was applied. A subset of patients received adjuvant therapy and was also included in this analysis. Finally, a total of 127 patients were included for this study.

\section{Follow-up and endpoints}

The main endpoint for this study was pathological response assessed at time of surgery. The amount of residual tumor was classified based on surgical pathology reports as pathologic complete response ( $\mathrm{PCR}$ ), microscopic residual disease (MRD) or gross residual disease (GRD). Percent residual tumor in the pathological sample was not available for this study. Three other clinical endpoints were included for this study including overall survival (OS), distant metastasis (DM) and in-field local recurrence (LR). The time associated to the endpoint was defined from treatment start date to date of first event. The last date of follow-up was used for patients with no events.

Follow-up chest CT scans with contrast (unless the patient had a contraindication to contrast, e.g. renal dysfunction or allergy) were performed every three to six months after treatment for patients at our institution based on US national guidelines ${ }^{13}$ to assess tumor progression.

\section{CT Acquisition and Segmentation}

Planning CTs were acquired according to scanning protocol at our institution using GE "lightspeed" CT scanner (GE Medical System, Milwaukee, WI, USA). Tumor segmentation was performed on radiation therapy planning CTs using Eclipse (Varian Medical System, Palo Alto, CA, USA). The primary tumor site was retrospectively contoured guided by existing treatment planning contours. Using both soft tissues and lung windows, air, vessels, normal tissue or surrounding organs were subsequently excluded from the contours (Figure 1.A). All contours were done by (T.P.C., V.A., Y.H.), and then all individually verified by an expert radiation oncologist (R.H.M.). 


\section{Features Extraction and Selection}

Radiomic features describing tumor phenotype were extracted $(m=1603)$ from the primary tumor site with an in-house Matlab 2013 (The Mathworks Inc., Natick, Massachusetts, United States) toolbox and the software 3D Slicer 4.4.0 ${ }^{21}$ (Figure 1.B). Average voxel spacing was $(0.9 \mathrm{~mm} \times 0.9 \mathrm{~mm} \times 3 \mathrm{~mm})$ respectively for $(x, y, z)$ and was resampled $3 \times 3 \times 3 \mathrm{~mm}^{3}$ prior to feature extraction to have standardized voxel spacing across the cohort. A bin width of 25 Hounsfield units (HU) was used for textural features. All features are described in the supplement of a previous study ${ }^{10}$.

Fifteen Radiomic features were selected based on stability and variance for this study (features selection is described in Supplement I). Additionally, we defined three conventional, pre-treatment, clinically utilized, volumetric features for comparison to advanced phenotypic features prior chemoradiation. These features consisted of tumor volume, 2D axial maximal diameter and 3D maximal diameter. 2D axial maximal diameter corresponds to the greatest diameter in the axial plane. 3D maximal diameter refers to the greatest diameter in any direction. All volume and diameter measurements were obtained from the primary tumor and did not include the sum diameters or volumes of involved lymph nodes.

A)

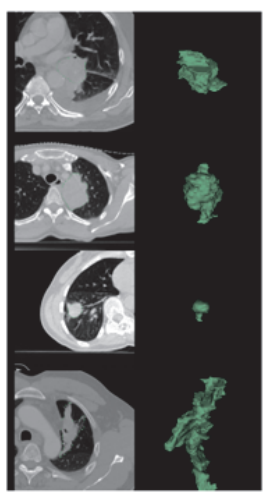

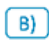

Resampled
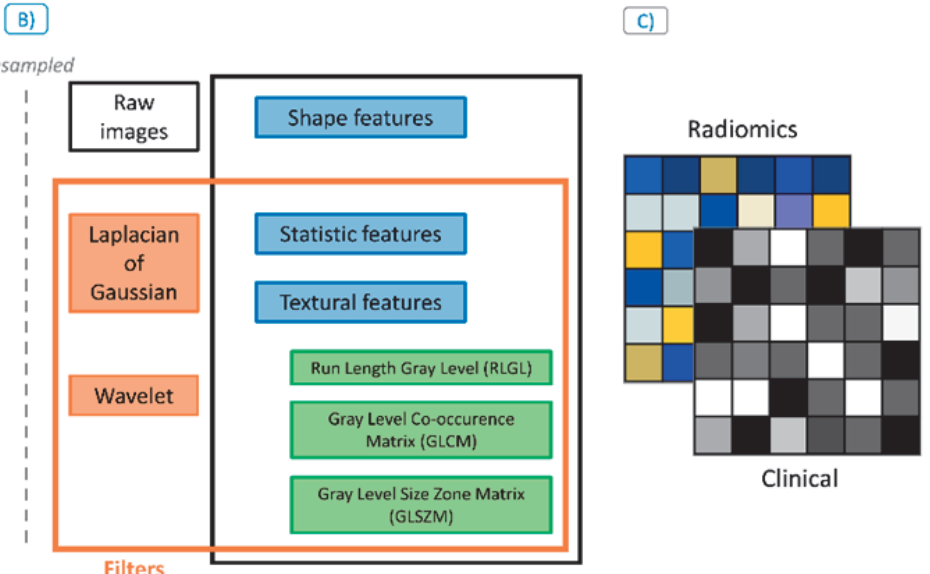

Figure 1. Radiomic analysis workflow description: A) Lung primary tumor site was manually contoured from treatment planning images (shown in green on CT image on the left and the 3D mask on the right). B) All images were subsequently resampled and from those contours the radiomic features describing tumor phenotype were extracted using three feature groups: Shape, Statistic and Textural features, with and without Wavelet and Laplacian of Gaussian filtering. C) Finally, association between radiomic features and the clinical outcomes were investigated for image-based biomarkers discovery. 


\section{Statistical Analysis}

All statistical analyses were done on $\mathrm{R}$ software ${ }^{22}$ version 3.1.3. Predictive performance of these remaining features were assessed using the "survcomp" package ${ }^{23,24}$ version 1.16 from Bioconductor ${ }^{25}$. We computed receiver operating characteristic (ROC) area under the curve (AUC) for binary outcomes. Predictive power was reported as proportional or disproportionate to the risk of experiencing the response as the feature value is increasing.

Difference for clinical categories was assessed using chi-square or two-sided Wilcoxontest respectively for categorical or continuous variables. Noether test was used to consider AUC significance from 0.5 (random). Survival and disease-free probability curve were computed using Kaplan-Meier analysis. A three year estimate was reported for the analysis. Log-rank test was used to assess difference in probability curves between pathological response groups. A p-value below 0.05 was considered as significant. Features with an AUC above 0.60 and a p-value below 0.05 were considered predictive.

Multivariate models were made using logistic regression for pathological response using the same subgroup for the univariate analysis to compare their performance. Three models were created with 1) Conventional (volume and axial/3D diameters), 2) Radiomics (predictive features for GRD) and 3) Combined (Conventional + radiomics) features.

We compare model performance with the validation AUC using the cross validation (CV). The cohort was split using $80 \%$ of the cohort for training and $20 \%$ for validation and using a 100 of iterations for the CV. Patients were randomized using a conservative random split using the "caret" package ${ }^{26}$. Difference between the CV models performance was done using a permutation test. The outcome labels were randomly resampled ( $k=1000$ times) and a new CV was computed for each random label combination. One-sided Wilcoxon test was computed for each random label models, the $W_{k}$ statistic extracted and compared to $W_{0}$ (true label) to assess if a model performance was significantly greater than another.

\section{RESULTS}

127 patients with NSCLC were included in this study. The median age was 60.5 years old (range 32.7 to 77.6 years old), with a majority of women (53.5\%) and Caucasian (92.1\%). Tumor histology was predominantly adenocarcinoma (56.6\%) and AJCC ${ }^{27}$ stage IIIA (75.6\%). The median follow-up was 41.8 months (range 2.7-117.2). The distribution of pathological response was 27 (21.3\%), 33 (26.0\%) and 67 (52.7\%) respectively for complete response, microscopic and gross residual disease.

All treatment information can be found in the Table 1. Comparison between pathological complete response ( $p C R$ ) versus microscopic (MRD) and gross (GRD) residual dis- 
ease, showed no significant differences between treatment ( $p$-value $=0.50$, Chi-square test) and radiation dose ( $p$-value=0.32). Significant differences were found between overall stages ( $p$-value=0.02) and histology ( $p$-value<0.001), likely driven by the fact that the distribution is skewed for histology and overall stage.

Table 1. Patient and treatment characteristics. Median (range) is reported for continuous and counts (percentage) for categorical variables. Statistical difference between complete pathological responders vs. noncomplete responders was computed using Chi-Square or Wilcoxon-test respectively for categorical and continuous variables.

\begin{tabular}{|c|c|c|c|c|}
\hline Variable / Group & $\begin{array}{l}\text { Median (Range) / } \\
\text { Count (\%) }\end{array}$ & $\mathrm{pCR}(\mathrm{n}=27)$ & MRD \& GRD $(n=100)$ & p-value \\
\hline Age [years] & $60.5(32.7-77.6)$ & $61.5(32.7-75.2)$ & $60.4(33.1-77.6)$ & 0.93 \\
\hline \multicolumn{5}{|l|}{ Performance Status } \\
\hline 0 & $60(47.2 \%)$ & $10(37.0 \%)$ & $50(50 \%)$ & \multirow[t]{3}{*}{0.33} \\
\hline 1 & $59(46.5 \%)$ & $14(51.9 \%)$ & $45(45 \%)$ & \\
\hline $2-3$ & $8(6.3 \%)$ & $3(11.1 \%)$ & $5(5 \%)$ & \\
\hline \multicolumn{5}{|l|}{ Gender } \\
\hline Female & $68(53.5 \%)$ & $11(40.7 \%)$ & $57(57 \%)$ & \multirow[t]{2}{*}{0.19} \\
\hline Male & $59(46.5 \%)$ & $16(59.2 \%)$ & $43(43 \%)$ & \\
\hline \multicolumn{5}{|l|}{ Ethnicity } \\
\hline White & 117 (92.1\%) & $25(92.6 \%)$ & $92(92 \%)$ & \multirow[t]{4}{*}{0.61} \\
\hline Black & $5(3.9 \%)$ & $1(3.7 \%)$ & $4(4 \%)$ & \\
\hline Hispanic & $3(2.4 \%)$ & $0(0 \%)$ & $3(3 \%)$ & \\
\hline Asian & $2(1.6 \%)$ & $1(3.7 \%)$ & $1(1 \%)$ & \\
\hline \multicolumn{5}{|l|}{ Histology } \\
\hline Adenocarcinoma & $72(56.6 \%)$ & $5(18.5 \%)$ & $67(67 \%)$ & \multirow[t]{4}{*}{$<0.001$} \\
\hline Squamous cell Carcinoma & $32(25.3 \%)$ & $14(51.8 \%)$ & $18(18 \%)$ & \\
\hline NSCLC & $18(14.2 \%)$ & $6(14.8 \%)$ & $12(12 \%)$ & \\
\hline Others $^{1}$ & $5(3.9 \%)$ & $2(7.4 \%)$ & $3(3 \%)$ & \\
\hline \multicolumn{5}{|l|}{ Overall stages } \\
\hline$\| \mathrm{A}$ & $2(1.5 \%)$ & $1(3.7 \%)$ & $1(1 \%)$ & \multirow[t]{4}{*}{0.02} \\
\hline IIB & $8(6.3 \%)$ & $5(18.5 \%)$ & $3(3 \%)$ & \\
\hline IIIA & $96(75.6 \%)$ & $18(66.7 \%)$ & $78(78 \%)$ & \\
\hline IIIB & $21(16.5 \%)$ & $3(11.1 \%)$ & $18(18 \%)$ & \\
\hline \multicolumn{5}{|l|}{ Treatment sequence } \\
\hline Concurrent & $107(84.3 \%)$ & $24(88.8 \%)$ & $83(83 \%)$ & \multirow[t]{3}{*}{0.49} \\
\hline Induction & $18(11.0 \%)$ & $2(7.4 \%)$ & $12(12 \%)$ & \\
\hline Others $^{2}$ & $2(1.7 \%)$ & $1(3.8 \%)$ & $1(1 \%)$ & \\
\hline Radiation Dose [Gy] & $54(45-70)$ & $54(46-70)$ & $54(45-70)$ & 0.32 \\
\hline \multicolumn{5}{|l|}{ Pathological response } \\
\hline $\mathrm{pCR}$ & $27(21.3 \%)$ & $27(100 \%)$ & $0(0 \%)$ & \multirow[t]{3}{*}{-} \\
\hline MRD & $33(26.0 \%)$ & $0(0 \%)$ & $33(33 \%)$ & \\
\hline GRD & $67(52.7 \%)$ & $0(0 \%)$ & $67(67 \%)$ & \\
\hline
\end{tabular}

Label: $\mathrm{pCR}=$ pathologic complete response, $\mathrm{MRD}=$ microscopic residual disease, $\mathrm{GRD}=$ gross residual disease. 1 Large Cell neuroendocrine carcinoma, Mixed NSCLC and SCLC, Adenoid cystic carcinoma, Sarcomatoid. 2 RT Only, Sequential. 
Relationship between clinical outcomes and pathological response subgroups was investigated (Table 2 ). The median (range) for overall survival, distant metastasis and local recurrence was respectively 41.8 (2.7-117.2), 10.8 (2.5-73.5) and 14 (4.7-66.5) months. No significant difference was observed for survival between pathological response ( $p$ value $=0.86$, Log-rank test). However, $p C R$ patients had significantly higher probabilities at three years for distant metastasis-free $(79 \%, p$-value $=0.036)$ and local recurrencefree $(93 \%$, p-value=0.013). Kaplan-Meier curves can be found in Figure S3 and concordance index for radiomics features in Figure S4 in Supplement II.

Table 2. Three years estimate from Kaplan-Meier survival curve for each pathological response subgroup. Difference between groups was assessed with Log-Rank test.

\begin{tabular}{lllll}
\hline Three years estimate probability & pCR $(n=27)$ & MRD $(n=33)$ & GRD $(n=67)$ & -value \\
\hline Overall Survival & $72 \%$ & $53 \%$ & $52 \%$ & 0.86 \\
Distant Metastasis Free & $79 \%$ & $59 \%$ & $50 \%$ & 0.036 \\
Local Recurrence Free & $94 \%$ & $75 \%$ & $62 \%$ & 0.013 \\
\hline
\end{tabular}

Label: $\mathrm{pCR}=$ pathologic complete response, $\mathrm{MRD}=$ microscopic residual disease, $\mathrm{GRD}=$ gross residual disease.

Table 3. Description of radiomic features with associated feature group and filter.

\begin{tabular}{|c|c|c|c|}
\hline Selected Radiomic feature & $\begin{array}{l}\text { Radiomic } \\
\text { group }\end{array}$ & $\begin{array}{l}\text { Filter } \\
\text { associated }\end{array}$ & Description \\
\hline Sphere Disproportionality & Shape & None & $\begin{array}{l}\text { Ratio between tumor area and a sphere with the same } \\
\text { volume as the tumor }\end{array}$ \\
\hline Root Mean Square & Statistics & Wavelet HLL & Root mean square of the voxels intensity value \\
\hline Range & Statistics & $\begin{array}{l}\text { Wavelet LLH } \\
\text { Wavelet LHH }\end{array}$ & The range of voxels intensity values \\
\hline Energy & Statistics & Wavelet HLL & Describe the energy of the image \\
\hline Mean & Statistics & Wavelet HLL & The mean voxel intensity value \\
\hline Kurtosis & Statistics & $\begin{array}{l}\text { LoG 3D - } \\
5 \mathrm{~mm}\end{array}$ & $\begin{array}{l}\text { Describe the shape of a probability distribution of the } \\
\text { voxel intensity histogram }\end{array}$ \\
\hline Skewness & Statistics & $\begin{array}{l}\text { LoG 3D - } \\
4 \mathrm{~mm}\end{array}$ & $\begin{array}{l}\text { Describe the shape of a probability distribution of the } \\
\text { voxel intensity histogram }\end{array}$ \\
\hline Correlation & GLCM & Wavelet LHH & Correlation of the GLCM matrix \\
\hline Entropy & GLCM & $\begin{array}{l}\text { LoG 3D - } \\
5 \mathrm{~mm}\end{array}$ & $\begin{array}{l}\text { Complexity of the GLCM matrix (sensitive to the number } \\
\text { of unique voxel patterns in the tumor) }\end{array}$ \\
\hline Homogeneity 2 & GLCM & $\begin{array}{l}\text { LoG 2D - } \\
4 \mathrm{~mm}\end{array}$ & $\begin{array}{l}\text { Homogeneity of voxels patterns (similar patterns across } \\
\text { the whole tumor) }\end{array}$ \\
\hline Cluster Prominence & GLCM & $\begin{array}{l}\text { LoG 3D - } \\
3 \mathrm{~mm}\end{array}$ & $\begin{array}{l}\text { Sensitive to flat zone (area of connecting voxel with } \\
\text { same value) }\end{array}$ \\
\hline $\begin{array}{l}\text { Low Intensity Large Area } \\
\text { Emphasis }\end{array}$ & GLSZM & Wavelet LHH & $\begin{array}{l}\text { Sensitive to flat zone with low intensity voxel (e.g. } \\
\text { necrotic area) }\end{array}$ \\
\hline Large Area Emphasis & GLSZM & $\begin{array}{l}\text { LoG 3D - } \\
5 \mathrm{~mm}\end{array}$ & Sensitive to flat zone \\
\hline $\begin{array}{l}\text { High Intensity Large Area } \\
\text { Emphasis }\end{array}$ & GLSZM & $\begin{array}{l}\text { LoG 3D - } \\
5 \mathrm{~mm}\end{array}$ & $\begin{array}{l}\text { Sensitive to flat zone with high intensity voxel (e.g. } \\
\text { calcifications, blood vessels) }\end{array}$ \\
\hline
\end{tabular}

Label: GLCM = Gray-level Co-occurrence Matrix, GLSZM = Gray-Level Size Zone Matrix, LoG=Laplacian of Gaussian, L=Low, H=High. 
Fifteen features were selected based on stability and variance (see Supplement I) and were evaluated for performance to predict clinical outcomes. Those features included one shape, seven statistics and seven textural features incorporated four gray-level cooccurrence matrix (GLCM) sensitive to voxel patterns and three gray-level size zone matrix (GLSZM) sensitive to flat zone (area of connecting voxel with same value) are described in Table 3.

Pathological response was our primary clinical endpoint. We first determined if radiomic features could identify tumors likely to respond poorly (GRD) vs. tumors likely to respond well ( $\mathrm{pCR}$ and $\mathrm{MRD}$ ) to the chemoradiation (Figure 2.A). The fifteen selected advanced imaging features had an AUC of 0.53 to 0.66 for GRD (described in Table S2 in Supplement II). Seven features were significantly predictive (range AUC 0.61-0.66, pvalue $<0.05$ ) for GRD. From those seven predictive features, two were risk proportionate as the feature value increase (GLCM entropy and stats root mean square) and five disproportionate (mean and skewness in voxel intensity histogram, spherical disproportionality and two GLSZM large area emphasis) to experience GRD. No conventional volumetric imaging features were significant from random or above at the threshold of AUC > 0.6 (range 0.57 to 0.59 , p-value $>0.05$ ) and all were disproportionate to the risk of GRD.

We then investigated the predictive power for identifying pathologic complete response $(\mathrm{pCR})$ vs. non-complete response (MRD and GRD). The AUC range of radiomic features (Figure 2.B) was 0.52-0.63 and 0.51-0.55 for conventional features (described in Table S3 in Supplement II). The best performing radiomic feature, Wavelet HLL mean, was also the only significantly predictive feature $(A \cup C=0.63, p$-value $=0.01$, Noether test $)$ and was risk proportional. No conventional imaging features were predictive for pCR (range 0.51 to 0.55 , all $p$-value $>0.05$ ).

Multivariate models were created for each set of features (Figure 3), including conventional ( 3 features), radiomics ( 7 predictive features for GRD) and the resulting combined set (10 features). The median AUC values of the cross validation were 0.57, 0.65 and 0.65 for GRD and $0.60,0.61$ and 0.68 for $\mathrm{pCR}$ respectively for conventional, radiomics and combined models. The combined and radiomics model for GRD performed significantly better on the cross validation compare to clinical features alone. For $p C R$, no significant difference was found between radiomics and conventional model performance. However, the combined model significantly outperformed both radiomics and conventional. 

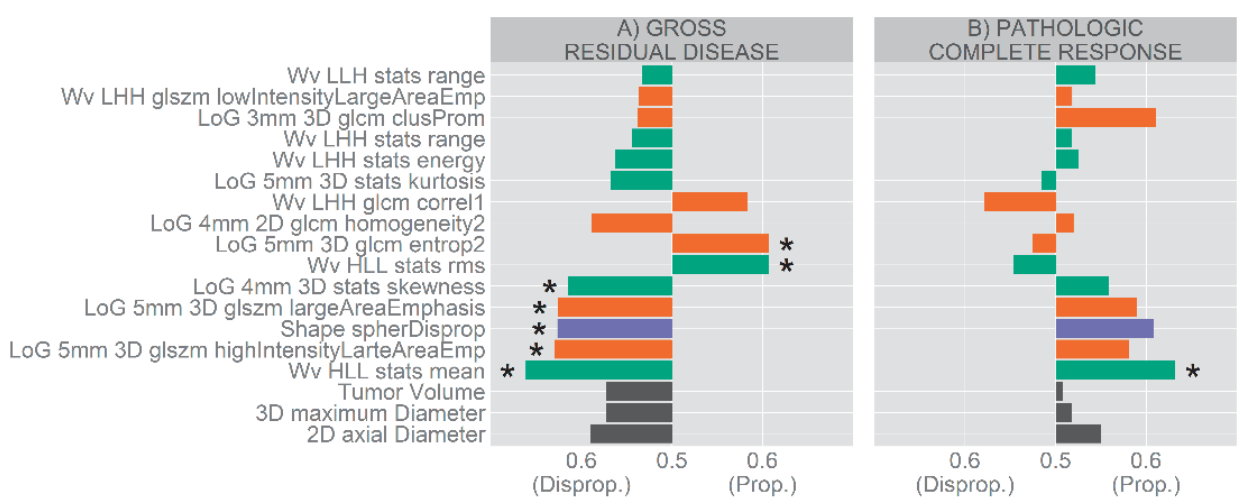

$\mathrm{AUC}$

\section{Statistics $\square$ Texture $\square$ Shape $\square$ Conventional}

Figure 2. AUC of Radiomic features and conventional volumetric imaging features for A) poor responders (gross residual disease) vs. good responders (pathologic complete response and microscopic residual disease) and B) pathological complete responders vs. non-complete responders (microscopic and gross residual disease). Predicting power was reported as proportional or disproportionate to the risk of experiencing the response as the feature value is increasing. Features reported with a "*" are significant from random (Noether test, $p$-value $<0.05)$. Legend colors indicates feature group.
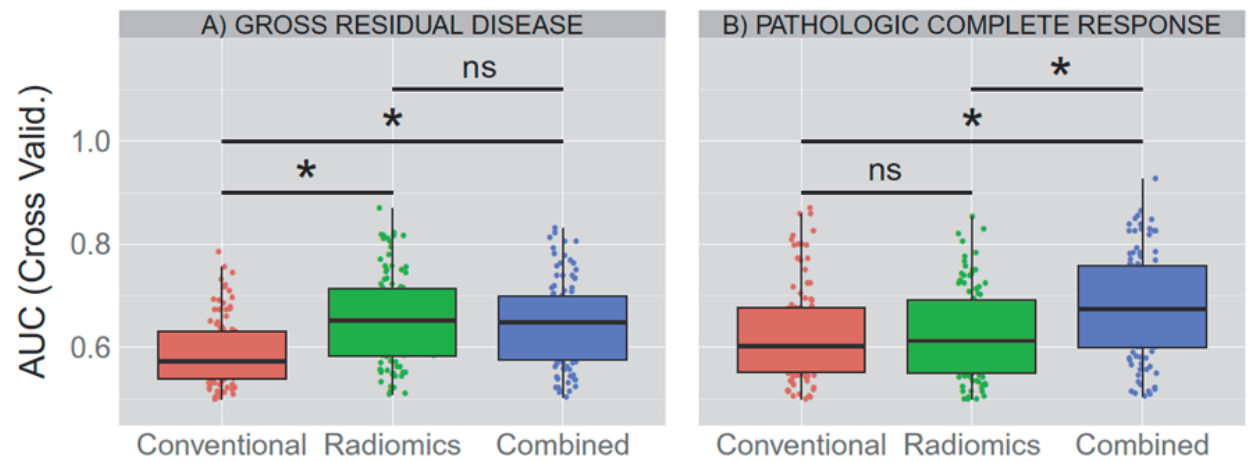

Figure 3. Comparison of multivariate models for A) Gross residual disease and B) complete pathological response. AUC from the validation is reported from the Cross-Validation (100 iterations, $80 \%$ training and 20\% validation) for each model. Comparison between models were done using a permutation test. $A$ "*” is reported if the model performance is significantly greater than the other, else "ns" for non-significant.

\section{DISCUSSION}

Radiomics ${ }^{1}$ is an emerging field of quantitative imaging that aims to extract phenotypic tumor information from clinical imaging data. In this study we demonstrate the predictive power of radiomic phenotypic features for pathological response in patients with NSCLC. Pathological response is a standard endpoint, assessed at time of surgery for a direct measure of neoadjuvant chemotherapy effect. Pathologic response was signifi- 
cantly associated with clinical outcomes in our study (distant metastasis and local recurrence) showing the importance of predicting pathological outcome. Using data mining techniques, we selected fifteen radiomics features based on stability and variance. From those features, we identify seven features predictive of pathological gross residual disease (GRD) and one feature predictive of pathological complete responders ( $p C R$ ). In contrast, no pre-treatment conventional clinically utilized features (volume or diameter) were predictive for pathological response. Lastly, the radiomics model also performed significantly better on a cross validation than the conventional model for GRD. A combined model using conventional and radiomics outperformed for $\mathrm{pCR}$.

The seven significant predictive features for GRD enabled us to represent phenotypic characteristics of a lung tumor that is less likely to respond to neoadjuvant chemoradiation. Spherical disproportionality (a measure of the similarity between the tumor and a sphere with an equivalent volume) of the primary tumor site was associated with pathological response (AUC above 0.6 for both GRD and $\mathrm{PCR}$ ). Remarkably, more complex tumors are likely to be associated with $\mathrm{PCR}$ while more spherical tumors are more likely to be associated with GRD. In comparison, conventional radiographic such as volume and diameter were not predictive for pathological response (neither PCR nor GRD), and may not capture sufficient shape information as advanced shape radiomic features. It is noteworthy that larger tumor dimension (both volume and diameter) appeared to be associated with pCR. Tumor with large flat zones (area of connecting voxels of similar intensity) were associated to PCR whereas tumor with rich complex patterns (heterogeneities) were associated to GRD. Finally based on the common significant predictor for both pCR and GRD (Wavelet HLL - mean) indicated that tumor with overall lower voxel intensity (darker) were associated with GRD after Wavelet HLL filtering. Multivariate performances demonstrated that radiomics information added complementary information for pCR (significantly greater performance for the combined model) and outperformed conventional features alone for GRD.

To our knowledge this is the first study investigating pathological response to neoadjuvant chemoradiation using advanced quantitative imaging features from CT images. A previous study from Ravanelli et al. ${ }^{28}$ investigated response of first line of chemotherapy (with no concurrent radiation therapy or surgery) for patients with advanced NSCLC using two textural features (uniformity and grey-level) from contrast enhanced CT images. They found an AUC of 0.74 for their multivariate model (Leave one out cross validation). Conventional features (tumor volume and diameters) have been identified as a prognostic factor in prior studies ${ }^{29-31}$. However patients in those reports did not receive surgical resection as part of their therapy as did patients in our study. Conventional features may thus be more relevant as a prognostic factor in the absence of surgical intervention. Additionally, we did not find any significant association between radiomics and clinical outcomes, supporting the idea that surgical resection of the tumor undermines image-based features prediction for clinical outcomes. We believe that the un- 
derlying tumor phenotype may be more relevant for identifying pathologic response than either volume or diameter, although these may remain valuable for assessing response to therapy. Other studies ${ }^{32,33}$ investigated the association of conventional features (tumor volume) but demonstrated a lack of correlation between measured tumor volume change and pathologic response in NSCLC, supporting the fact that none of our conventional features were significant.

Limitations of this study include the cohort size $(n=127)$. Due to a large number of available radiomic features and concern for multiple testing, dimension reduction was used with restrictive criteria to perform drastic selection, therefore excluding potential predictive features for any clinical outcomes. However despite this very restrictive datadriven approach selection (about 1\% remaining after), we were able to find predictive features. Additionally, the incidence of $p C R(n=27)$ was much lower than GRD $(n=60)$, potentially explaining the differences in the predictive power for pathological complete response. Since the study period was conducted over 12 years, we recognize that there is heterogeneity in the treatments delivered to the patient cohort. However, we did not find any association between radiation dose, cycles of chemotherapy or other treatment characteristics and pathologic response. Thus, we believe the results presented remain valid despite treatment heterogeneity. A major limitation of radiomics is the lack of standardization in image acquisition that without access to raw images is difficult to control and hence standardize patient cohorts. Yet, despite the noise introduced by variation in acquisition protocols, radiomic models have shown consistent and reproducible association with outcomes in multiple independent datasets ${ }^{7,9-11}$. Furthermore, we have previously demonstrated the reproducibility ${ }^{7}$ of the radiomics features using the RIDER ${ }^{34}$ test / retest dataset and we resampled of imaging using a common pixel spacing $(3 \times 3 \times 3 \mathrm{~mm} 3)$ to limit variability across patients. Finally, we acknowledge the limitations of the clinical applicability of this study. This study was initiated to find potential patterns in tumor phenotype that could predict pathological response prior to the start of therapy. Although several features were significantly predictive for pathological endpoints, we were unable to identify subgroups associated with OS, LRR or DR using radiomic features. Despite the need for further validation sets, this study provides a basis for additional research (e.g using PET-CT features) that could improve performance. We believe such radiomics based analyses can be used as a complementary method of patient stratification for NSCLC prior to the initiation of therapy as is currently being investigated in other disease sites such as breast ${ }^{35,36}$, colorectal cancer ${ }^{37}$, and glioblastoma $^{38}$.

In conclusion, we identified CT-based radiomic features predictive for pathological response in patient with locally advanced NSCLC who previously received trimodality therapy. This study demonstrates that radiomics can provide additional phenotypic information that may reflect underlying tumor sensitivity to chemoradiation. Predicting pathological response prior to initiation of neoadjuvant chemoradiation has significant 
potential clinical applications such as developing adaptive therapy based on pretreatment tumor phenotype. These radiomic features which can be captured from clinically-available imaging modalities performed better than conventionally reported metrics.

\section{ACKNOWLEDGMENTS}

Authors acknowledge financial support from the National Institute of Health (NIH-USA U24CA194354, and NIH-USA U01CA190234). This project was partially funded by the Kaye Scholar Award and the Brigham and Women's Hospital Department of Radiation Oncology Clinical Translational Grant. 


\section{REFERENCES}

1. Lambin P, Rios-Velazquez E, Leijenaar R, Carvalho S, van Stiphout RGPM, Granton P, Zegers CML, Gillies R, Boellard R, Dekker A, Aerts HJWL. Radiomics: Extracting more information from medical images using advanced feature analysis. European Journal of Cancer. 2012;48(4):441-446.

2. Kumar V, Gu Y, Basu S, Berglund A, Eschrich SA, Schabath MB, Forster K, Aerts HJWL, Dekker A, Fenstermacher D, Goldgof DB, Hall LO, Lambin P, Balagurunathan Y, Gatenby RA, et al. Radiomics: the process and the challenges. Magnetic Resonance Imaging. 2012;30(9):1234-1248.

3. Gillies RJ, Kinahan PE, Hricak H. Radiomics: Images Are More than Pictures, They Are Data. Radiology. 2015:151169.

4. Parmar C, Rios Velazquez E, Leijenaar R, Jermoumi M, Carvalho S, Mak RH, Mitra S, Shankar BU, Kikinis R, Haibe-Kains B, Lambin P, Aerts HJWL. Robust Radiomics Feature Quantification Using Semiautomatic Volumetric Segmentation. Woloschak GE, ed. PLOS ONE. 2014;9(7):e102107.

5. Rios Velazquez E, Aerts HJWL, Gu Y, Goldgof DB, De Ruysscher D, Dekker A, Korn R, Gillies RJ, Lambin P. A semiautomatic CT-based ensemble segmentation of lung tumors: Comparison with oncologists' delineations and with the surgical specimen. Radiotherapy and Oncology. 2012;105(2):167-173.

6. Leijenaar RTH, Carvalho S, Velazquez ER, van Elmpt WJC, Parmar C, Hoekstra OS, Hoekstra CJ, Boellaard R, Dekker ALAJ, Gillies RJ, Aerts HJWL, Lambin P. Stability of FDG-PET Radiomics features: An integrated analysis of test-retest and inter-observer variability. Acta Oncologica. 2013;52(7):1391-1397.

7. Aerts HJWL, Velazquez ER, Leijenaar RTH, Parmar C, Grossmann P, Cavalho S, Bussink J, Monshouwer R, Haibe-Kains B, Rietveld D, Hoebers F, Rietbergen MM, Leemans CR, Dekker A, Quackenbush J, et al. Decoding tumour phenotype by noninvasive imaging using a quantitative radiomics approach. Nature Communications. 2014;5.

8. Parmar C, Grossmann P, Bussink J, Lambin P, Aerts HJWL. Machine Learning methods for Quantitative Radiomic Biomarkers. Scientific Reports. 2015;5:13087.

9. Leijenaar RT, Carvalho S, Hoebers FJ, Aerts HJ, van Elmpt WJ, Huang SH, Chan B, Waldron JN, O'sullivan $B$, Lambin P. External validation of a prognostic CT-based radiomic signature in oropharyngeal squamous cell carcinoma. Acta Oncologica. 2015; (ahead-of-print):1-7.

10. Coroller TP, Grossmann P, Hou Y, Rios Velazquez E, Leijenaar RTH, Hermann G, Lambin P, Haibe-Kains B, Mak RH, Aerts HJWL. CT-based radiomic signature predicts distant metastasis in lung adenocarcinoma. Radiotherapy and Oncology. 2015;114(3):345-350.

11. Parmar C, Leijenaar RTH, Grossmann P, Rios Velazquez E, Bussink J, Rietveld D, Rietbergen MM, HaibeKains B, Lambin P, Aerts HJWL. Radiomic feature clusters and Prognostic Signatures specific for Lung and Head \&amp; Neck cancer. Scientific Reports. 2015;5:11044.

12. Siegel R, Ma J, Zou Z, Jemal A. Cancer statistics, 2014: Cancer Statistics, 2014. CA: A Cancer Journal for Clinicians. 2014;64(1):9-29.

13. National Comprehensive Cancer Network. NCCN guidelines: Non-Small Cell Lung Cancer (Version 7.2015). 2015.

14. Albain KS, Swann RS, Rusch VW, Turrisi AT, Shepherd FA, Smith C, Chen Y, Livingston RB, Feins RH, Gandara DR, Fry WA, Darling G, Johnson DH, Green MR, Miller RC, et al. Radiotherapy plus chemotherapy with or without surgical resection for stage III non-small-cell lung cancer: a phase III randomised controlled trial. The Lancet. 2009;374(9687):379-386.

15. van Meerbeeck JP, Kramer GWPM, Van Schil PEY, Legrand C, Smit EF, Schramel F, Tjan-Heijnen VC, Biesma B, Debruyne C, van Zandwijk N, Splinter TAW, Giaccone G. Randomized Controlled Trial of Resection Versus Radiotherapy After Induction Chemotherapy in Stage IIIA-N2 Non-Small-Cell Lung Cancer. JNCl Journal of the National Cancer Institute. 2007;99(6):442-450.

16. Hellmann MD, Chaft JE, William WN, Rusch V, Pisters KM, Kalhor N, Pataer A, Travis WD, Swisher SG, Kris $M G$, others. Pathological response after neoadjuvant chemotherapy in resectable non-small-cell lung cancers: proposal for the use of major pathological response as a surrogate endpoint. The Lancet Oncology. 2014;15(1):e42-e50. 
17. Mouillet G, Monnet E, Milleron B, Puyraveau M, Quoix E, David P, Ducoloné A, Molinier O, Zalcman G, Depierre A, Westeel V, Intergroupe Francophone de Cancérologie Thoracique (IFCT). Pathologic complete response to preoperative chemotherapy predicts cure in early-stage non-small-cell lung cancer: combined analysis of two IFCT randomized trials. Journal of Thoracic Oncology: Official Publication of the International Association for the Study of Lung Cancer. 2012;7(5):841-849.

18. Isobe K, Hata Y, Sakaguchi S, Sato F, Takahashi S, Sato K, Sano G, Sugino K, Sakamoto S, Takai Y, Mitsuda A, Terahara A, Shibuya K, Takagi K, Homma S. Pathological response and prognosis of stage III non-small cell lung cancer patients treated with induction chemoradiation. Asia-Pacific Journal of Clinical Oncology. 2012;8(3):260-266.

19. Eisenhauer EA, Therasse P, Bogaerts J, Schwartz LH, Sargent D, Ford R, Dancey J, Arbuck S, Gwyther S, Mooney M, Rubinstein L, Shankar L, Dodd L, Kaplan R, Lacombe D, et al. New response evaluation criteria in solid tumours: Revised RECIST guideline (version 1.1). European Journal of Cancer. 2009;45(2):228247.

20. Werner-Wasik M, Xiao Y, Pequignot E, Curran WJ, Hauck W. Assessment of lung cancer response after nonoperative therapy: tumor diameter, bidimensional product, and volume. A serial CT scan-based study. International Journal of Radiation Oncology* Biology* Physics. 2001;51(1):56-61.

21. Pieper S, Halle M, Kikinis R. 3D Slicer. In: IEEE International Symposium on Biomedical Imaging: Nano to Macro, 2004.; 2004:632-635 Vol. 1.

22. Anon. $R$ Core Team (2013). R: A language and environment for statistical computing. $R$ Foundation for Statistical Computing, Vienna, Austria. ISBN 3-900051-07-0, URL http://www.R-project.org/.

23. Haibe-Kains B, Desmedt C, Sotiriou C, Bontempi G. A comparative study of survival models for breast cancer prognostication based on microarray data: does a single gene beat them all? Bioinformatics. 2008;24(19):2200-2208.

24. Schröder MS, Culhane AC, Quackenbush J, Haibe-Kains B. survcomp: an R/Bioconductor package for performance assessment and comparison of survival models. Bioinformatics. 2011;27(22):3206-3208.

25. Gentleman RC, Carey VJ, Bates DM, Bolstad B, Dettling M, Dudoit S, Ellis B, Gautier L, Ge Y, Gentry J, Hornik K, Hothorn T, Huber W, lacus S, Irizarry R, et al. Bioconductor: open software development for computational biology and bioinformatics. Genome Biology. 2004;5(10):R80.

26. Kuhn M. Building predictive models in $\mathrm{R}$ using the caret package. Journal of Statistical Software. 2008;28(5):1-26.

27. Anon. American Joint Committee on Cancer. AJCC Cancer Staging Manual, 7th ed. New York: Springer 2010.

28. Ravanelli M, Farina D, Morassi M, Roca E, Cavalleri G, Tassi G, Maroldi R. Texture analysis of advanced non-small cell lung cancer (NSCLC) on contrast-enhanced computed tomography: prediction of the response to the first-line chemotherapy. European Radiology. 2013;23(12):3450-3455.

29. Bradley JD, leumwananonthachai N, Purdy JA, Wasserman TH, Lockett MA, Graham MV, Perez CA. Gross tumor volume, critical prognostic factor in patients treated with three-dimensional conformal radiation therapy for non-small-cell lung carcinoma. International Journal of Radiation Oncology* Biology* Physics. 2002;52(1):49-57.

30. Alexander BM, Othus M, Caglar HB, Allen AM. Tumor Volume Is a Prognostic Factor in Non-Small-Cell Lung Cancer Treated With Chemoradiotherapy. International Journal of Radiation Oncology*Biology*Physics. 2011;79(5):1381-1387.

31. Stinchcombe TE, Morris DE, Moore DT, Bechtel JH, Halle JS, Mears A, Deschesne K, Rosenman JG, Socinski MA. Post-chemotherapy gross tumor volume is predictive of survival in patients with stage III nonsmall cell lung cancer treated with combined modality therapy. Lung Cancer (Amsterdam, Netherlands). 2006;52(1):67-74.

32. Cerfolio RJ, Bryant AS, Winokur TS, Ohja B, Bartolucci AA. Repeat FDG-PET After Neoadjuvant Therapy is a Predictor of Pathologic Response in Patients With Non-Small Cell Lung Cancer. The Annals of Thoracic Surgery. 2004;78(6):1903-1909. 
33. Poettgen C, Theegarten D, Eberhardt W, Levegruen S, Gauler T, Krbek T, Stamatis G, Teschler H, Kuehl H, Bockisch A, Stuschke M. Correlation of PET/CT Findings and Histopathology after Neoadjuvant Therapy in Non-Small Cell Lung Cancer. Oncology. 2007;73(5-6):316-323.

34. Zhao B, James LP, Moskowitz CS, Guo P, Ginsberg MS, Lefkowitz RA, Qin Y, Riely GJ, Kris MG, Schwartz LH. Evaluating Variability in Tumor Measurements from Same-day Repeat CT Scans of Patients with NonSmall Cell Lung Cancer 1. Radiology. 2009;252(1):263-272.

35. Pickles MD, Lowry M, Gibbs P. Pretreatment Prognostic Value of Dynamic Contrast-Enhanced Magnetic Resonance Imaging Vascular, Texture, Shape, and Size Parameters Compared With Traditional Survival Indicators Obtained From Locally Advanced Breast Cancer Patients: Investigative Radiology. 2016;51(3):177-185.

36. Fox MJ, Gibbs P, Pickles MD. Minkowski functionals: An MRI texture analysis tool for determination of the aggressiveness of breast cancer. Journal of magnetic resonance imaging: JMRI. 2015.

37. Lubner MG, Stabo N, Lubner SJ, Del Rio AM, Song C, Halberg RB, Pickhardt PJ. CT textural analysis of hepatic metastatic colorectal cancer: pre-treatment tumor heterogeneity correlates with pathology and clinical outcomes. Abdominal Imaging. 2015;40(7):2331-2337.

38. Lee J, Jain R, Khalil K, Griffith B, Bosca R, Rao G, Rao A. Texture Feature Ratios from Relative CBV Maps of Perfusion MRI Are Associated with Patient Survival in Glioblastoma. AJNR. American journal of neuroradiology. 2016;37(1):37-43. 


\section{Chapter}

\section{Radiomic-based Pathological Response Prediction from Primary Tumours and Lymph Nodes in NSCLC}

Published in: Journal of Thoracic Oncology (2016)

Radiomic-based Pathological Response Prediction from Primary Tumors and Lymph Nodes in NSCLC Thibaud Coroller, Vishesh Agrawal, Elizabeth Huynh, Vivek Narayan, Stephanie Lee, Raymond Mak*, Hugo Aerts* 


\section{ABSTRACT}

Introduction: Non-invasive biomarkers that capture the total tumor burden could provide important complementary information for precision medicine to aid clinical decision-making. We investigated the value of radiomic data, extracted from pre-treatment computed tomography (CT) images of the primary tumor and lymph nodes, in predicting pathological response following neoadjuvant chemoradiation prior to surgery.

Methods: Eighty-five patients with resectable locally-advanced (stage II-III) non-small cell lung cancer (NSCLC) (median age: 60.3 years; 65\% female) treated from 2003-2013 were included in this IRB-approved study. Radiomics analysis was performed on 85 primary tumors and 178 lymph nodes to discriminate between pathological complete response $(p C R)$ or gross residual disease (GRD). Twenty non-redundant and stable features (10 from each site) were evaluated using the area under the curve (AUC) (all pvalues were corrected for multiple hypothesis testing). Classification performance of each feature set was evaluated using random forest and nested cross validation.

Results: Three radiomic features (describing primary tumor sphericity and lymph node homogeneity) were significantly predictive of $\mathrm{PCR}$ with similar performances (all $A \cup C=0.67, p$-value<0.05). Those two features (quantifying lymph node homogeneity) were predictive of GRD (AUC range: $0.72-0.75$, p-value<0.05) and performed significantly better than the primary features ( $A \cup C=0.62$ ). Multivariate analysis showed that for $\mathrm{PCR}$, the radiomics feature set alone had the best performing classification (median $A \cup C=0.68$ ). Furthermore, for GRD classification, combining radiomic and clinical data significantly outperformed all other feature sets (median AUC=0.73).

Conclusion: Lymph node phenotypic information was significantly predictive for pathological response and showed higher classification performance than radiomic features obtained from the primary tumor. 


\section{INTRODUCTION}

For non-small cell lung cancer (NSCLC) patients treated with trimodality therapy (chemoradiation followed by surgery), pathological response is a direct measure of therapeutic response that is assessed at the time of surgery. Pathological response can be used as a surrogate marker to aid clinical decision-making. However, the benefits of adding surgery to chemoradiation for stage IIIA NSCLC remains unclear ${ }^{1,2}$. Therefore, non-invasive early predictors of pathological response are needed to identify patients who would most likely benefit from continuing chemoradiation versus proceeding to surgery.

Increasingly, image-based biomarkers have been investigated for use in clinical decisionmaking ${ }^{3}$. In the field of quantitative imaging, radiomics ${ }^{4,5}$ is an emerging discipline that aims to non-invasively characterize the tumor phenotype using a robust highthroughput extraction of descriptive features from medical images ${ }^{6,7}$. Ultimately, these features have been used to develop imaging-based biomarkers to provide complementary information to clinical and genomic data for association with clinical outcomes. Most of these image-based biomarkers have been developed solely based on analyses of patient primary tumors ${ }^{8-25}$.

In locally-advanced NSCLC, tumors have a propensity to spread from the primary site to lymph nodes. These involved lymph nodes could have distinct phenotypic characteristics related to biological processes affecting disease spread and thus, treatment response. Given that knowledge, there has also been investigation into the textural features of tumors outside of the primary site. CT textural features were recently shown to be correlated with malignant and benign lymph nodes ${ }^{26,27}$. However, to date there have been very few quantitative analyses of NSCLC lymph node features correlated with clinical outcomes. A study from Li et al. ${ }^{28}$ that used conventional positron emission tomography (PET) features (standard uptake values and volume) demonstrated that adding lymph node information to the primary tumor features increased their predictive performance for overall disease relapse in NSCLC. Analysis of phenotypic characteristics of lymph nodes may be particularly relevant for NSCLC, as lymph node involvement in locally advanced NSCLC is an important predictor of patient treatment outcome 29-31.

Here, we investigated the value of radiomic data, extracted from pre-treatment computed tomography (CT) images of the primary tumor and lymph nodes, in predicting pathological response following neoadjuvant chemoradiation prior to surgery. This study has important implications for the development of non-invasive biomarkers that will capture the total tumor burden and provide important complementary information for precision medicine to aid clinical decision-making. 


\section{METHOD}

\section{Patient selection}

One hundred and seventy-seven patients with stage II-III NSCLC treated at our institution between 2003 and 2013 with neoadjuvant chemotherapy and radiotherapy (chemoradiation) prior to surgical resection were included in this Institutional Review Board (IRB)-approved study. This cohort included a subset of patients who received adjuvant chemotherapy. No exclusion based on histology was applied.

Patients without surgical pathology reports were excluded, leaving 167 patients. Eight patients were excluded for a delay in surgery greater than 120 days after the completion of chemoradiation. An additional 32 patients were excluded due to a lack of CT imaging or original treatment contours. Clinically involved lymph nodes prior to neoadjuvant therapy were identified by fludeoxyglucose positron emission tomography (FDGPET) $(n=82)$ or cervical mediastinoscopy $(n=71)$. Finally, 42 patients without clinically involved lymph nodes or nodes that were indistinguishable from the primary tumor were excluded, resulting in a total of 85 patients that were included in this study.

\section{Follow-up and endpoints}

Pathological response was assessed at the time of surgery. The amount of residual tumor was classified based on surgical pathology reports as pathologic complete response $(p C R)$, microscopic residual disease (MRD), or gross residual disease (GRD). The percent of residual tumor in the pathological sample was not available for this study due to limited pathologic reports available at the time of analysis. Follow-up chest CT scans with contrast (unless the patient had a contraindication to contrast, e.g. renal dysfunction) were performed every three to six months after treatment based on US national guidelines $^{32}$ to assess tumor progression.

Three other clinical endpoints included in this study were overall survival (OS), distant metastasis (DM) and in-field (within the planning target volume (PTV) of the primary tumor and/or nodal sites) local recurrence (LRR). Local control was considered as freedom from LRR. The time associated with the endpoint was defined from the treatment start date to the date of the first event (or last date of follow-up for patients with no events).

\section{CT acquisition and segmentation}

CTs were acquired according to standardized scanning protocols at our institution using a GE "Lightspeed" CT scanner (GE Medical System, Milwaukee, WI, USA) for treatment planning. Tumor segmentation was performed on radiation therapy planning CTs using 
Eclipse software (Varian Medical System, Palo Alto, CA, USA). The primary tumor site and lymph nodes were contoured using both soft tissue and lung windows by the treating radiation oncologists. Air, vessels, normal tissue or surrounding organs were subsequently excluded from these contours, and then individually verified by an expert radiation oncologist. If a patient presented with more than one clinically positive nodal station, the union of all the stations was analyzed.

\section{Feature extraction and selection}

Phenotypic radiomic features were extracted from both primary tumors and lymph nodes (Figure 1.A) with an in-house Matlab 2013 (The Mathworks Inc., Natick, Massachusetts, United States) toolbox and 3D Slicer 4.4.0 ${ }^{33}$. Voxels were resampled $\left(3 \times 3 \times 3 \mathrm{~mm}^{3}\right)$ prior to feature extraction to standardize the voxel spacing across the cohort. Additionally, a voxel intensity bin width of 25 Hounsfield units (HU) was used for textural features to increase their sensitivity relative to the raw image. A description of the radiomic features can be found in the supplement material from a previous study ${ }^{8}$.

Features were selected based on stability and variance, resulting in twenty (10 from the primary site and 10 from the lymph nodes). The feature selection method can be found in Supplement I. The selected features, which showed no redundancy (Figure S4), are individually described in Table 1.

The performances of radiomic features were then compared against conventional and clinical variables. The two conventional features included in the study were the primary tumor maximum axial diameter and the total primary tumor and lymph node(s) volume. The nine clinical variables included were age, gender, race, performance status, overall stage, $\mathrm{T}$-stage, $\mathrm{N}$-stage, radiation dose and chemotherapy sequence.

\section{Statistical analysis}

All statistical analyses were performed in R software ${ }^{34}$ version 3.2.4. A "one vs all" multiclass resolution was used to create two groups of pathological response (Figure 1.B): A) patients likely to respond well to neoadjuvant chemoradiation ( $p C R$ vs. non-PCR (MRD and GRD)) and B) patients likely to respond poorly to neoadjuvant chemoradiation (GRD vs. non-GRD ( $p C R$ and MRD)).

The statistical difference between clinical variables for $\mathrm{pCR}$ vs. non-pCR was assessed using a Chi-square or two-sided Wilcoxon test for categorical or continuous variables, respectively. Survival estimates were calculated using the Kaplan-Meier method and controlled using a log-rank test. The three-year survival or event-free probabilities are reported for each pathological response subgroup ( $\mathrm{CCR}, \mathrm{MRD}$ or GRD). The predictive performance of the radiomic features was assessed using the "survcomp" package ${ }^{35}$. 
The area under the receiver operating characteristic curve (AUC) was computed and reported for each feature as directly or inversely proportional to the risk of experiencing the event (e.g. GRD) as the feature value increased. AUCs from primary tumor and lymph node features are denoted as $A \cup C_{\text {Prim }}$ and $A \cup C_{L N}$, respectively. Noether's test was used to compute the $p$-value to evaluate the significance of the AUC from random $(A \cup C=0.5)$. P-values were adjusted for multiple hypothesis testing using the false discovery rate (FDR) method ${ }^{36}$. Features with an AUC above 0.60 and FDR corrected $p$ value below 0.05 were considered predictive.
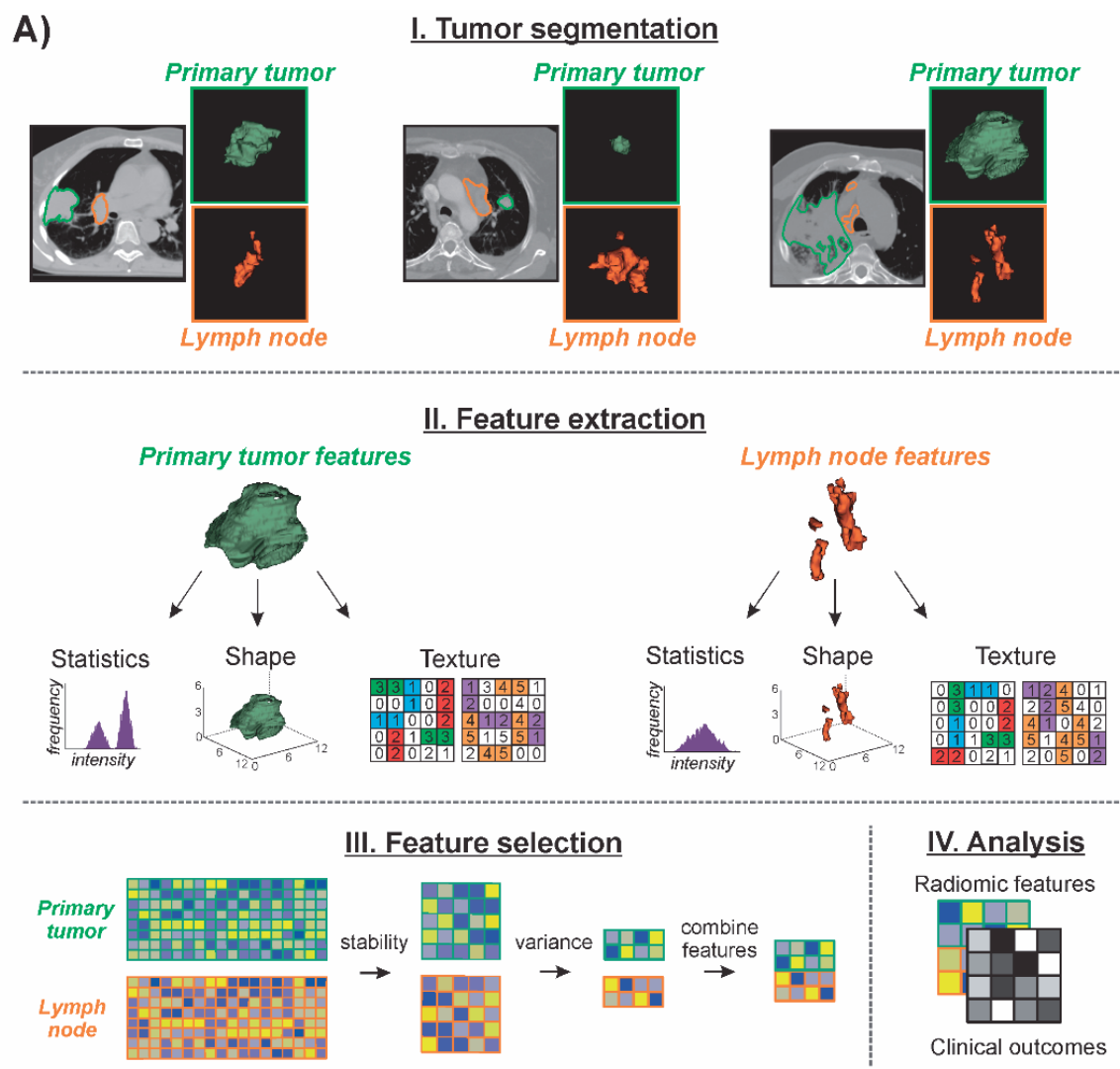

B)

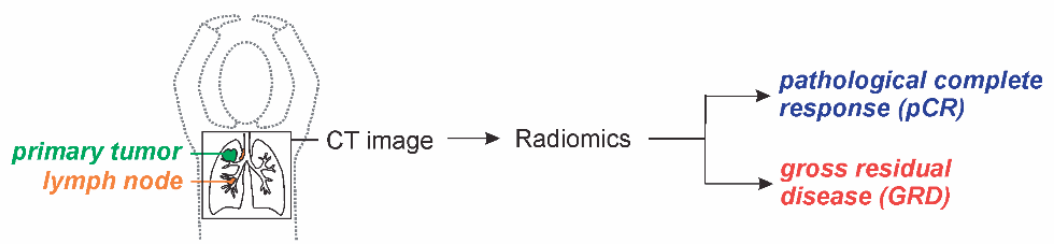

Figure 3. A) Schematic of the radiomics quantification workflow demonstrating feature extraction from the lung primary tumor site (in green) and lymph nodes (in orange) from pre-treatment CT images. B) Radiomics could enable precision medicine by classifying patients prior to therapy based on how they will respond to chemoradiation. 
Table 1. Description of the selected radiomic features with their associated feature group and filter.

\begin{tabular}{|c|c|c|c|c|}
\hline Radiomic feature & Site & $\begin{array}{l}\text { Radiomic } \\
\text { group }\end{array}$ & Associated filter & Description \\
\hline Sphere Sphericity & $P$ & Shape & none & $\begin{array}{l}\text { How close the tumor is to a sphere with } \\
\text { similar volume }\end{array}$ \\
\hline Mean & LN & Stats & Wavelet LLL & Mean voxel intensity value \\
\hline Total Energy & $P$ & Stats & Wavelet LHH & Energy of the image \\
\hline Root mean square & $\mathrm{P}$ & Stats & Wavelet LHH & $\begin{array}{l}\text { Root mean square of the voxel intensity } \\
\text { values }\end{array}$ \\
\hline Variance & LN & Stats & Wavelet $\mathrm{HHH}$ & Variance of the voxel intensity histogram \\
\hline Maximum Probability & $\mathrm{P} / \mathrm{LN}$ & GLCM & None & $\begin{array}{l}\text { Most frequent voxel intensity pattern in the } \\
\text { tumor }\end{array}$ \\
\hline Entropy & $P$ & GLCM & 3D LoG $5 \mathrm{~mm}$ & $\begin{array}{l}\text { Complexity of the GLCM matrix (sensitive to } \\
\text { the number of unique voxel patterns in the } \\
\text { tumor) }\end{array}$ \\
\hline Cluster Prominence & LN & GLCM & None & $\begin{array}{l}\text { Sensitive to flat zones (areas of connecting } \\
\text { voxels with the same value) }\end{array}$ \\
\hline Correlation & LN & GLCM & $\begin{array}{l}\text { Wavelet LHL } \\
/ \text { LHL }\end{array}$ & $\begin{array}{l}\text { Correlation of the GLCM matrix (smooth } \\
\text { gradients in the patterns) }\end{array}$ \\
\hline $\begin{array}{l}\text { Informational measure } \\
\text { of correlation }\end{array}$ & $P$ & GLCM & $\begin{array}{l}\text { Wavelet LLL } \\
\text { / 3D LoG } 5 \mathrm{~mm}\end{array}$ & $\begin{array}{l}\text { Generalization of the classical correlation } \\
\text { coefficient of a normal distribution }\end{array}$ \\
\hline Homogeneity & $P$ & GLCM & 3D LoG $5 \mathrm{~mm}$ & $\begin{array}{l}\text { Sensitive to similar patterns of voxels across } \\
\text { the whole tumor }\end{array}$ \\
\hline $\begin{array}{l}\text { Large Area Emphasis } \\
(\mathrm{LAE})\end{array}$ & $\mathrm{P}$ & GLSZM & 3D LoG 5 mm & $\begin{array}{l}\text { Sensitive to large flat zones (areas of } \\
\text { connecting voxels with the same value) }\end{array}$ \\
\hline $\begin{array}{l}\text { Low Intensity Large } \\
\text { Area Emphasis (LILAE) }\end{array}$ & $\mathrm{P}$ & GLSZM & Wavelet HLH & $\begin{array}{l}\text { Sensitive to flat zones with low intensity } \\
\text { voxels (e.g. areas of necrosis) }\end{array}$ \\
\hline $\begin{array}{l}\text { High intensity Large } \\
\text { Area Emphasis (HILRE) }\end{array}$ & LN & GLSZM & $\begin{array}{l}\text { 3D LoG } 5 \text { mm / } \\
\text { Wavelet LHH }\end{array}$ & $\begin{array}{l}\text { Sensitive to flat zones with high intensity } \\
\text { voxels }\end{array}$ \\
\hline $\begin{array}{l}\text { Gray Level Non } \\
\text { Uniformity (GLN) }\end{array}$ & LN & GLCM & 3D LoG $5 \mathrm{~mm}$ & $\begin{array}{l}\text { Measures the similarity of gray level values } \\
\text { throughout the image. A smaller GLN is } \\
\text { expected if the gray level values are similar } \\
\text { throughout the image }\end{array}$ \\
\hline Zone percentage (ZP) & LN & GLSZM & 2D LoG $4 \mathrm{~mm}$ & $\begin{array}{l}\text { Sum of unique discrete flat zone intensity } \\
\text { values divided by the number of voxels (the } \\
\text { lower the value, the more homogeneous) }\end{array}$ \\
\hline
\end{tabular}

Label: $P=$ Primary, $L N=$ Lymph Nodes, $L O G=$ Laplacian of Gaussian, GLCM = Gray-level Co-occurrence Matrix, GLSZM = Gray-Level Size Zone Matrix, RLGL = Run Length Gray-Level, L = Low, $\mathrm{H}=$ High.

Classifications were made using the random forest method from the "randomForest" package ${ }^{37}$. We compared the performances of the models using nested cross validation (CV) and returned the AUC (any values below 0.5 were flipped). The cohort was split with 70\% used for model tuning and training, and the remaining 30\% for validation in 100 iterations. Patients were randomized using a conservative random split using the "caret" package ${ }^{38}$. Models were generated from the clinical, conventional, radiomics or combined (radiomics + clinical) dataset.

Significant differences between the performances of the CV models were evaluated using a permutation test. Only a non-exhaustive pairwise list of four hypotheses was 
investigated 1) Combined $>$ radiomics, 2) Combined $>$ clinical, 3) Radiomics $>$ clinical and 4) Radiomics > conventional. Outcome labels were randomly resampled ( $k=100$ permutations) and a new CV was computed for each random label combination. A onesided Wilcoxon test was computed for each random label model, the $W_{k}$ statistic was extracted and compared to the $W_{0}$ (true label) to assess if a model's performance was significantly greater than the other. P-values were defined as:

$$
p=\frac{1}{n_{\text {permut }}+1} \sum_{k=1}^{n_{\text {permut }}}\left\{\begin{array}{l}
\text { if } W_{k}>W_{0}=1 \\
\text { if } W_{k}<W_{0}=0
\end{array}\right.
$$

\section{RESULTS}

\section{Clinical characteristics}

Eighty-five patients with primary tumor and lymph node involvement were included in this study (Table 2). A total number of 85 primary sites and 178 lymph nodes were contoured. The patient cohort was $65 \%$ female, exhibited predominantly adenocarcinoma histology (62\%), and had a median age of 60 years (range 33-76). The median number of clinically involved lymph node stations was 2 (range 1-8) and the majority of patients presented with stage III disease (82.4\% IIIA and $15.3 \% \mathrm{IIIB}$ ) at the time of diagnosis. The sequence of chemotherapy administration with radiotherapy included 74 concurrent and 10 induction followed by concurrent chemotherapy. The median radiation dose was 54 Gy (range: 45-70 Gy).

The pathological response of patients included 13 (15.3\%) complete responders (pCR), 24 (28.2\%) with microscopic disease (MRD) and 48 (56.5\%) with gross residual disease (GRD). No significant associations were found between patient, tumor or treatment variables with pathological response (Table 2 ) except for mean lymph node station volume ( $p$-value $=0.01$, Wilcoxon test). However, total lymph node stations volume was not significantly different between pathological response ( $p$-value $=0.22$ ). In particular, the association between complete pathological response and pre-operative treatment sequencing $(p$-value $=0.83$, Chi-square test) or radiation dose $(p$-value $=0.14)$ was not significant. 
Table 2. Patient and treatment characteristics. Median (range) is reported for continuous variables and counts (percentage) for categorical variables.

\begin{tabular}{|c|c|c|c|c|}
\hline Variable / Group & Total $(n=85)$ & $\mathrm{pCR}(\mathrm{n}=13)$ & MRD / GRD (n=72) & $p$-value \\
\hline Age [years] & $60.3(32.7-75.9)$ & $61.5(32.7-75.2)$ & 59.76 (33.1-75.9) & 0.96 \\
\hline \multicolumn{5}{|l|}{ Performance Status } \\
\hline 0 & $37(43.5 \%)$ & $5(38.5 \%)$ & $32(44.4 \%)$ & \multirow[t]{2}{*}{0.92} \\
\hline $1-2$ & $48(56.5 \%)$ & $8(61.5 \%)$ & $40(55.6 \%)$ & \\
\hline \multicolumn{5}{|l|}{ Gender } \\
\hline Female & $55(65 \%)$ & $7(53.8 \%)$ & $48(66.6 \%)$ & \multirow[t]{2}{*}{0.56} \\
\hline Male & $30(35 \%)$ & $6(46.2 \%)$ & $24(33.4 \%)$ & \\
\hline \multicolumn{5}{|l|}{ Ethnicity } \\
\hline White & $78(91.8 \%)$ & $13(100 \%)$ & $65(90.3 \%)$ & \multirow[t]{4}{*}{0.72} \\
\hline Black & $3(3.5 \%)$ & $0(0 \%)$ & $3(4.2 \%)$ & \\
\hline Hispanic & $3(3.5 \%)$ & $0(0 \%)$ & $3(4.2 \%)$ & \\
\hline Asian & $1(1.2 \%)$ & $0(0 \%)$ & $1(1.3 \%)$ & \\
\hline \multicolumn{5}{|l|}{ Histology } \\
\hline Adenocarcinoma & $53(62.4 \%)$ & $4(30.7 \%)$ & $49(68.1 \%)$ & \multirow[t]{4}{*}{0.05} \\
\hline Squamous cell Carcinoma & $20(23.5 \%)$ & $6(46.2 \%)$ & $14(19.4 \%)$ & \\
\hline NOS (large cell) & $10(11.8 \%)$ & $3(32.1 \%)$ & $7(9.7 \%)$ & \\
\hline Others $^{1}$ & $2(2.3 \%)$ & $0(0 \%)$ & $2(2.8 \%)$ & \\
\hline Number of Lymph node stations & $2(1-8)$ & $2(1-4)$ & $3(1-8)$ & 0.06 \\
\hline $\begin{array}{l}\text { Total Lymph nodes stations } \\
\text { volume }[\mathrm{cc}]\end{array}$ & $10.02(0.54-65.58)$ & $10.10(2.72-65.58)$ & $9.34(0.54-56.92)$ & 0.22 \\
\hline $\begin{array}{l}\text { Mean Lymph nodes stations } \\
\text { volume }[\mathrm{cc}]\end{array}$ & $4.20(0.30-48.30)$ & $6.50(2.70-48.30)$ & $3.95(0.30-16.60)$ & 0.01 \\
\hline \multicolumn{5}{|l|}{ Overall stage } \\
\hline$\| A$ & $2(2.4 \%)$ & $1(7.7 \%)$ & $1(1.4 \%)$ & \multirow[t]{4}{*}{0.11} \\
\hline $\mathrm{IIB}$ & $0(0 \%)$ & $0(0 \%)$ & $0(0 \%)$ & \\
\hline IIIA & $70(82.3 \%)$ & $12(92.3 \%)$ & $58(80.6 \%)$ & \\
\hline IIIB & $13(15.3 \%)$ & $0(0 \%)$ & $13(18 \%)$ & \\
\hline \multicolumn{5}{|c|}{ Pre-operative chemotherapy sequence } \\
\hline Concurrent & $74(87.1 \%)$ & $12(92.3 \%)$ & $62(86.1 \%)$ & \multirow[t]{3}{*}{0.83} \\
\hline Concurrent + Induction & $10(11.8 \%)$ & $1(7.7 \%)$ & $9(12.5 \%)$ & \\
\hline None (RT only) & $1(1.1 \%)$ & $0(0 \%)$ & $1(1.4 \%)$ & \\
\hline Radiation Dose [Gy] & $54(45-70)$ & $54(54-70)$ & $54(45-70)$ & 0.14 \\
\hline
\end{tabular}

Label: $\mathrm{pCR}=$ pathologic complete response, $\mathrm{MRD}=$ microscopic residual disease, $\mathrm{GRD}=$ gross residual disease, NOS $=$ not otherwise specified. ${ }^{1}$ Adenosquamous carcinoma, Adenoid cystic carcinoma

\section{Survival analysis}

The median follow-up time was 40.2 months (range: 2.7 to 117 months). The association between pathological response and outcomes was assessed using the Kaplan-Meier method and the three-year event-free probabilities are reported in Table 3. Although 
the p-values were not significant, there was a trend (Figure S5 in Supplement II) in the association between pathological response and freedom from distant metastasis (DM), where better pathologic response corresponded to a greater 3-year estimate of freedom from DM ( $\mathrm{pCR}=92 \%, \mathrm{MRD}=52 \%$ and $\mathrm{GRD}=44 \%$, Log-rank test, $\mathrm{p}$-value $=0.051$ ). A similar trend was observed between pathological response and local control, but was still not statistically significant ( $\mathrm{PCR}=91 \%, \mathrm{MRD}=65 \%$ and $\mathrm{GRD}=55 \%$, $\mathrm{p}$-value $=0.35$ ). Grouping the pathological responses into pCR vs. non-pCR (MRD and GRD) identified a statistically significant association for freedom from DM ( $p C R=92 \%$, non-pCR $=43 \%, p-$ value $=0.017$ ). Radiomic features were not predictive of any of the clinical outcomes in this dataset (Figure S6).

Table 3. Three-year estimates from the Kaplan-Meier survival curves for each pathological response subgroup. The global differences between groups were assessed with the Log-Rank test.

\begin{tabular}{lllll}
\hline Three-year estimate probability & $\mathrm{pCR}(\mathrm{n}=13)$ & $\mathrm{MRD}(\mathrm{n}=24)$ & $\mathrm{GRD}(\mathrm{n}=48)$ & $\mathrm{p}$-value \\
\hline Overall Survival & $82 \%$ & $48 \%$ & $58 \%$ & 0.17 \\
Freedom from Distant Metastasis & $92 \%$ & $52 \%$ & $44 \%$ & 0.051 \\
Local control & $91 \%$ & $65 \%$ & $55 \%$ & 0.35 \\
\hline
\end{tabular}

Label: $\mathrm{PCR}=$ pathologic complete response, $\mathrm{MRD}=$ microscopic residual disease, $\mathrm{GRD}=$ gross residual disease.

\section{Radiomics analysis of pathological response}

First, we investigated the predictive power of the radiomic features for patients likely to respond well to neoadjuvant chemoradiation ( $p C R$ vs. non-pCR). The area under the receiver operating characteristics curves (AUCS) for all radiomic features ranged from 0.51 to 0.75 . Two lymph node features were predictive of $p C R$ (range: 0.72 to $0.75, p$ value $=0.01$ ) (Figure 2.A). The best radiomics feature extracted from the lymph nodes $\left(A \cup C_{L N}=0.75\right)$ was significantly higher ( $p$-value $=0.03$, permutation test) than the best from primary site $\left(A \cup C_{\text {prim }}=0.61\right)$. The two predictive lymph node features were high intensity large area emphasis (HILAE) and zone percentage (ZP), and were both derived from the same underlying texture group, Gray Level Size Zone Matrix (GLSZM). None of the conventional features were predictive for pCR. Specifically, the total primary tumor and lymph node volume $(A \cup C=0.58)$ and primary tumor axial diameter $(A \cup C=0.56)$ were not significantly more predictive than random ( $p$-values $=0.47$ ).

Secondly, we investigated patients likely to respond poorly to neoadjuvant chemoradiation (GRD vs. non-GRD). The radiomic feature AUCs ranged from 0.50 to 0.67 (Figure 2.B). Three features were predictive for GRD (all three AUCs $=0.67$, $p$-values $=0.02$ ), including lymph node features $Z P$ and HILAE, which were also predictive for $p C R$. The third feature was sphericity of the primary tumor. The AUCs for the conventional features were moderately better for GRD (range: 0.60 to 0.61 ) than $\mathrm{pCR}$; however none of the conventional features were significantly more predictive than random ( $p$-values $=$ 0.09). All results can be found in detail in Table S1 and Table S2 (Supplement II). Addi- 
tionally, consistent results were found in subset analysis with patients with pathologically confirmed lymph node involvement via cervical mediastinoscopy ( $n=71$, Figure 57 ) or overall stage III only ( $\mathrm{n}=83$, Figures $\mathrm{S} 8$ ).

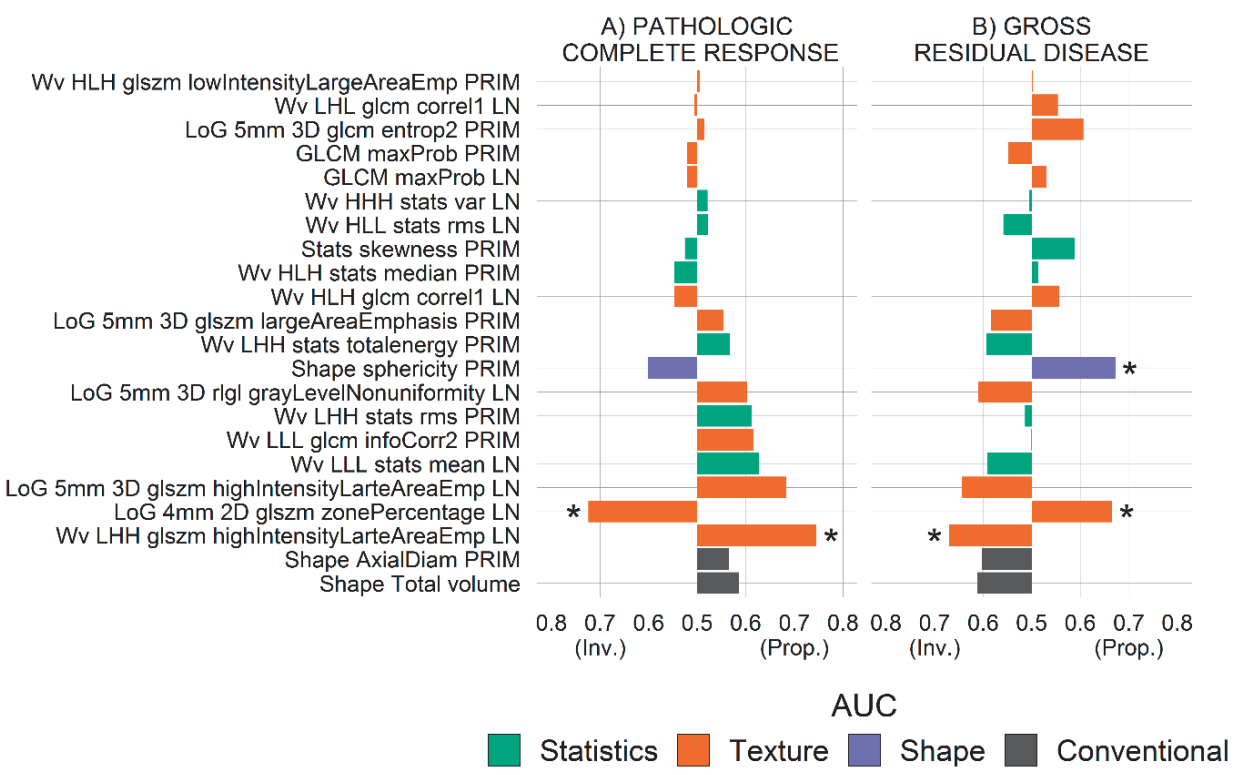

Figure 4. AUCs of selected radiomic and conventional imaging features for $A$ ) pathological complete response ( $p C R$ vs. non-pCR) and B) gross residual disease (GRD vs. non-GRD). Selected features are derived from the primary tumor (PRIM) or lymph node(s) (LN). The predictive power was reported as directly (Prop.) or inverseIy (Inv.) proportional to the risk of experiencing the event as the feature value is increased. Features reported with a "*" are significant from a random guess (Noether's test, p-value $\leq 0.05$, FDR corrected). Legend colors indicate feature groups.

HILAE is sensitive to large flat zones (connecting voxels of similar intensity) and is associated with the filter Wavelet LHH, which highlights fine and coarse textures. Higher HILAE values represent a more homogenous texture. Similarly, ZP is sensitive to the ratio between the flat zone total intensity value and the total number of voxels in the flat zone. ZP is associated with the filter LoG $4 \mathrm{~mm} \mathrm{2D}$, which highlights coarse textures. Lower ZP values represent fewer discrete intensity levels accessed in the region of interest, and thus a more homogeneous tumor. For $\mathrm{pCR}$, HILAE was directly proportional to risk and ZP was inversely proportional to risk, indicating an association between homogeneous lymph node texture and $\mathrm{pCR}$. The risk was symmetrical to $\mathrm{PCR}$ for these two features for GRD, with more heterogeneous lymph nodes associated with GRD. Moreover, a rounder primary tumor (quantified by its sphericity) was also predictive of GRD and directly proportional to the probability of GRD. It is noteworthy that two out of the three unique predictive features were extracted from the lymph nodes. Conventional features were all inversely proportional for GRD, indicating a trend that smaller tumors could be associated with poor response to neoadjuvant chemoradiation. However, 
none of the conventional features were significant ( $p$-values were 0.09 and 0.047 for pCR and GRD, respectively).

\section{Models classification performance}

To evaluate the performance of our models in classifying patients according to their pathological response, we used the random forest method. For pCR classification (Figure 3.A), the median AUC values were 0.62 for conventional, 0.64 for clinical, 0.68 for radiomics and 0.65 for combined (radiomics and clinical). The radiomics models performed significantly better than conventional models ( $p$-value $=0.05$, permutation test). No other significant differences were found between the performances of the other models ( $p$-values ranged from 0.24 to 0.92 ).

For GRD classification (Figure 3.B), the median AUC values were 0.60 for conventional, 0.69 for clinical, 0.71 for radiomics, and 0.73 for combined. The combined model had the highest median performance and performed significantly better than the radiomics $(p$-value $=0.01)$ and clinical $(p$-value $=0.05)$ models. The radiomics models alone performed better than the conventional models ( $p$-value $=0.03$ ) but not better than the clinical models ( $p$-value $=0.19$ ).

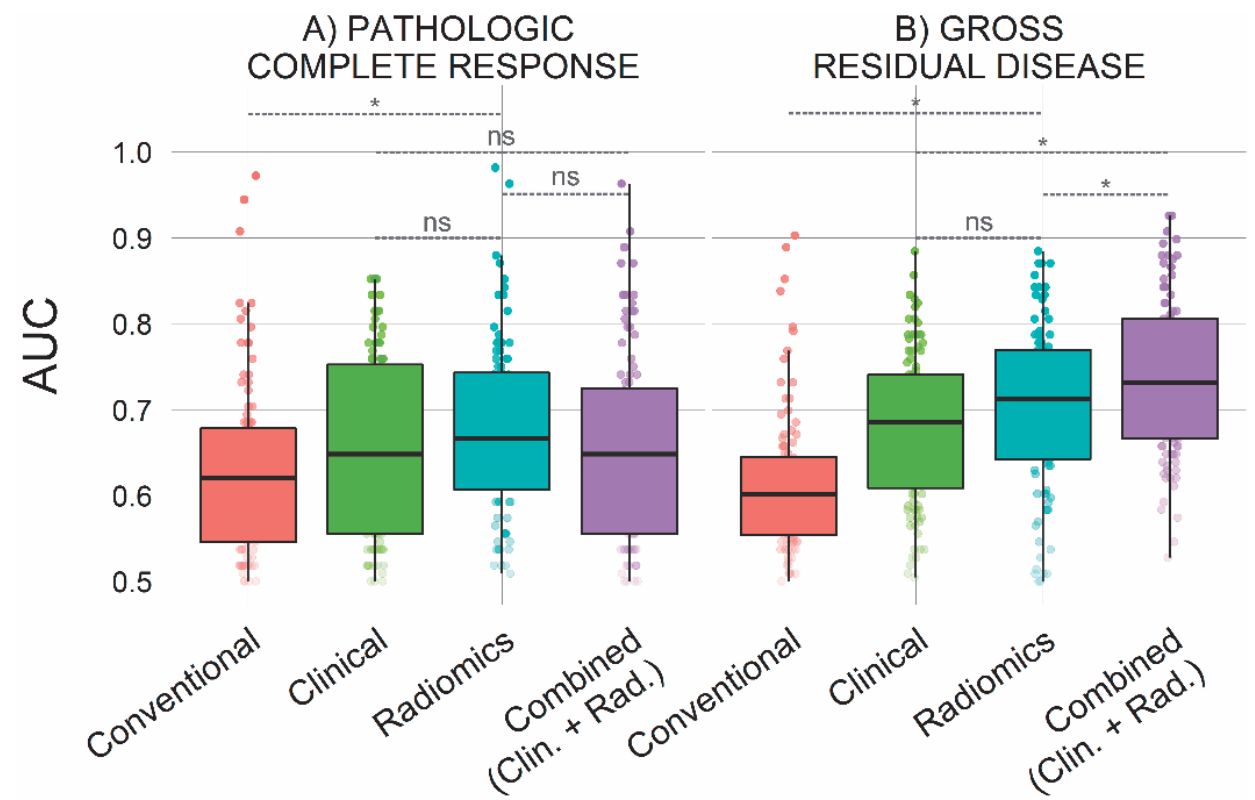

Figure 3 The performances of random forest classification of models for A) pathological complete response $(p C R)$ and $B$ ) gross residual disease (GRD). AUCs are reported from nested cross validation analysis. "*" indicates significant difference between the feature models (permutation test, $p$-value $\leq 0.05$ ) 


\section{DISCUSSION}

Radiomics ${ }^{4,5}$ is a rising field of quantitative imaging that aspires to capture tumor phenotype characteristics non-invasively using advanced imaging features. Radiomics may have a critical role in precision medicine, as it can predict clinical endpoints in patients prior to treatment ${ }^{8,10}$. Precision medicine is particularly important for stage III nonsmall cell lung cancer (NSCLC) patients, where it is known that multiple forms of local therapy (radiation or surgery) is not beneficial for all patients. However, currently, there is a lack of methods to predict how each patient will respond prior to treatment. Therefore, in this study, we sought to apply radiomics for prediction of pathological response using radiomic features extracted from pre-treatment images of primary tumors and lymph nodes in NSCLC patients. Furthermore, this is the first study, to our knowledge, that investigates the prediction of tumor response using lymph node imaging features.

Using data mining techniques independent from the outcomes and multiple testing correction, we found two radiomic features that were predictive for pathological complete responders ( $p C R$ ) and three for gross residual disease (GRD). In comparison, clinically used tumor measurements (diameter and volume) were poor predictors of pathological response. This potentially reveals the value of radiomics in predicting tumor susceptibility to chemoradiation. The radiomic features indicated that the sphericity of the primary tumor and homogeneity of the lymph nodes were predictive of pathological response. In addition, lymph node homogeneity has previously been shown to be associated with lymph node status (benign vs. malignant) in a study by Bayanati et al. ${ }^{26}$. Our results complement this study by demonstrating that homogeneous texture, even within a known malignancy, is further associated with better pathological response. These results show that the lymph node phenotype may contain valuable information for predicting clinical endpoints.

To validate these results, we generated classification models using a random forest method based on each feature set. We found that a radiomics model performed significantly better than all other models in predicting PCR and a model that combines clinical and radiomics data was the best at predicting GRD. In contrast, models built from conventional imaging features performed poorly, demonstrating that the size of the lymph nodes and primary tumor was not associated with pathological response. This data suggests that pre-treatment conventional imaging assessments or stratification by size or overall stage may not be sufficiently predictive of therapeutic response. Moreover, there is more useful quantitative data to be extracted from medical images than what is currently used clinically.

Despite a large number of studies investigating the relationship between tumor phenotype and clinical outcomes ${ }^{8-18}$, few studies have investigated the potential benefit of adding lymph node information ${ }^{26-28}$. Such lymph node information may be particularly 
relevant for patients with locally advanced NSCLC. Multiple clinical trials have shown that pathologic lymph node clearance is a strong predictor of outcome. Albain et al. ${ }^{1}$ demonstrated in a phase II clinical trial that patients with lymph node clearance had an overall survival of 34.4 months compared to 19 months for patients with residual lymph node disease at the time of surgery. Similarly, Suntharalingam et al. ${ }^{31}$ showed a 2-year overall survival rate of $75 \%$ for patients with mediastinal lymph node clearance versus $52 \%$ for patients with residual lymph node disease. Given that this study demonstrates an association between quantitative imaging features and pathological response, such features may aid the identification of patients who are more likely to have an incomplete local and regional tumor response.

Limitations of this study include the small sample size. Only patients with both lymph node (178) and primary tumor (85) contours available, and corresponding surgical pathology reports were included, which greatly limited the number of patients in this study. Our study nevertheless includes a larger cohort than previous lymph node studies $^{26-28}$ (our study had 85 patients, while other studies had 25 to 46 patients). An additional limitation for image analysis is the lack of standardization in image acquisition that, without access to raw images, is difficult to control. Yet, despite noise introduced by variations in acquisition protocols, radiomic models have shown consistent and reproducible associations with outcomes in multiple independent datasets ${ }^{8-11}$. Additionally, we resampled the images using a common pixel spacing to limit variability across patients and selected stable features using a test/retest assessment from the RIDER ${ }^{39}$ dataset. Finally, our cohort included small number of clinical overall stage II or nonpathological confirmed lymph nodes. We showed in the supplementary information that our findings were consistent for each subset.

This study demonstrates that the lymph node phenotype can present important information that reflects the underlying patient sensitivity to chemoradiation with a significantly superior power than that offered by the primary tumor site alone. Therefore, radiomic features captured from clinically-available imaging modalities offer predictive power superior to conventionally reported metrics. Predicting tumor response prior to initiation of neoadjuvant chemoradiation has potentially significant clinical implications for precision medicine, such as developing adaptive therapy plans based on pretreatment phenotypes.

\section{ACKNOWLEDGMENTS}

We acknowledge financial support from the National Cancer Institute (NIH). 


\section{REFERENCES}

1. Albain KS, Swann RS, Rusch VW, Turrisi AT, Shepherd FA, Smith C, Chen Y, Livingston RB, Feins RH, Gandara DR, Fry WA, Darling G, Johnson DH, Green MR, Miller RC, et al. Radiotherapy plus chemotherapy with or without surgical resection for stage III non-small-cell lung cancer: a phase III randomised controlled trial. The Lancet. 2009;374(9687):379-386.

2. van Meerbeeck JP, Kramer GWPM, Van Schil PEY, Legrand C, Smit EF, Schramel F, Tjan-Heijnen VC, Biesma B, Debruyne C, van Zandwijk N, Splinter TAW, Giaccone G. Randomized Controlled Trial of Resection Versus Radiotherapy After Induction Chemotherapy in Stage IIIA-N2 Non-Small-Cell Lung Cancer. JNCl Journal of the National Cancer Institute. 2007;99(6):442-450.

3. Lambin P, van Stiphout RGPM, Starmans MHW, Rios-Velazquez E, Nalbantov G, Aerts HJWL, Roelofs E, van Elmpt W, Boutros PC, Granone P, Valentini V, Begg AC, De Ruysscher D, Dekker A. Predicting outcomes in radiation oncology--multifactorial decision support systems. Nature reviews. Clinical oncology. 2013;10(1):27-40.

4. Lambin P, Rios-Velazquez E, Leijenaar R, Carvalho S, van Stiphout RGPM, Granton P, Zegers CML, Gillies R, Boellard R, Dekker A, Aerts HJWL. Radiomics: Extracting more information from medical images using advanced feature analysis. European Journal of Cancer. 2012;48(4):441-446.

5. Gillies RJ, Kinahan PE, Hricak H. Radiomics: Images Are More than Pictures, They Are Data. Radiology. 2015:151169.

6. Parmar C, Rios Velazquez E, Leijenaar R, Jermoumi M, Carvalho S, Mak RH, Mitra S, Shankar BU, Kikinis R, Haibe-Kains B, Lambin P, Aerts HJWL. Robust Radiomics Feature Quantification Using Semiautomatic Volumetric Segmentation. Woloschak GE, ed. PLOS ONE. 2014;9(7):e102107.

7. Rios Velazquez E, Aerts HJWL, Gu Y, Goldgof DB, De Ruysscher D, Dekker A, Korn R, Gillies RJ, Lambin P. A semiautomatic CT-based ensemble segmentation of lung tumors: Comparison with oncologists' delineations and with the surgical specimen. Radiotherapy and Oncology. 2012;105(2):167-173.

8. Coroller TP, Grossmann P, Hou Y, Rios Velazquez E, Leijenaar RTH, Hermann G, Lambin P, Haibe-Kains B, Mak RH, Aerts HJWL. CT-based radiomic signature predicts distant metastasis in lung adenocarcinoma. Radiotherapy and Oncology. 2015;114(3):345-350.

9. Parmar C, Leijenaar RTH, Grossmann P, Rios Velazquez E, Bussink J, Rietveld D, Rietbergen MM, HaibeKains B, Lambin P, Aerts HJWL. Radiomic feature clusters and Prognostic Signatures specific for Lung and Head \&amp; Neck cancer. Scientific Reports. 2015;5:11044.

10. Aerts HJWL, Velazquez ER, Leijenaar RTH, Parmar C, Grossmann P, Cavalho S, Bussink J, Monshouwer R, Haibe-Kains B, Rietveld D, Hoebers F, Rietbergen MM, Leemans CR, Dekker A, Quackenbush J, et al. Decoding tumour phenotype by noninvasive imaging using a quantitative radiomics approach. Nature Communications. 2014;5.

11. Leijenaar RT, Carvalho S, Hoebers FJ, Aerts HJ, van Elmpt WJ, Huang SH, Chan B, Waldron JN, O'sullivan $\mathrm{B}$, Lambin P. External validation of a prognostic CT-based radiomic signature in oropharyngeal squamous cell carcinoma. Acta Oncologica. 2015;(ahead-of-print):1-7.

12. Parmar C, Grossmann P, Bussink J, Lambin P, Aerts HJWL. Machine Learning methods for Quantitative Radiomic Biomarkers. Scientific Reports. 2015;5:13087.

13. Fried DV, Tucker SL, Zhou S, Liao Z, Mawlawi O, Ibbott G, Court LE. Prognostic Value and Reproducibility of Pretreatment CT Texture Features in Stage III Non-Small Cell Lung Cancer. International Journal of Radiation Oncology*Biology*Physics. 2014.

14. Davnall F, Yip CSP, Ljungqvist G, Selmi M, Ng F, Sanghera B, Ganeshan B, Miles KA, Cook GJ, Goh V. Assessment of tumor heterogeneity: an emerging imaging tool for clinical practice? Insights into Imaging. 2012;3(6):573-589.

15. Cunliffe A, Armato SG, Castillo R, Pham N, Guerrero T, Al-Hallaq HA. Lung Texture in Serial Thoracic Computed Tomography Scans: Correlation of Radiomics-based Features With Radiation Therapy Dose and Radiation Pneumonitis Development. International Journal of Radiation Oncology*Biology*Physics. 2015. 
16. Chae H-D, Park CM, Park SJ, Lee SM, Kim KG, Goo JM. Computerized Texture Analysis of Persistent PartSolid Ground-Glass Nodules: Differentiation of Preinvasive Lesions from Invasive Pulmonary Adenocarcinomas. Radiology. 2014:132187.

17. Parmar C, Grossmann P, Rietveld D, Rietbergen MM, Lambin P, Aerts HJWL. Radiomic Machine-Learning Classifiers for Prognostic Biomarkers of Head and Neck Cancer. Frontiers in Oncology. 2015;5.

18. Yip SSF, Coroller TP, Sanford NN, Huynh E, Mamon H, Aerts HJWL, Berbeco RI. Use of registration-based contour propagation in texture analysis for esophageal cancer pathologic response prediction. Physics in Medicine and Biology. 2016;61(2):906-922.

19. Yip SS, Coroller TP, Sanford NN, Mamon H, Aerts HJ, Berbeco RI. Relationship between the temporal changes in positron-emission-tomography-imaging-based textural features and pathologic response and survival in esophageal cancer patients. Frontiers in Oncology. 2016;6:72.

20. Win T, Miles KA, Janes SM, Ganeshan B, Shastry M, Endozo R, Meagher M, Shortman RI, Wan S, Kayani I, Ell PJ, Groves AM. Tumor Heterogeneity and Permeability as Measured on the CT Component of PET/CT Predict Survival in Patients with Non-Small Cell Lung Cancer. Clinical Cancer Research. 2013;19(13):3591-3599.

21. Coroller TP, Agrawal V, Narayan V, Hou Y, Grossmann P, Lee SW, Mak RH, Aerts HJWL. Radiomic phenotype features predict pathological response in non-small cell lung cancer. Radiotherapy and Oncology. 2016.

22. Huynh E, Coroller TP, Narayan V, Agrawal V, Hou Y, Romano J, Franco I, Mak RH, Aerts HJWL. CT-based radiomic analysis of stereotactic body radiation therapy patients with lung cancer. Radiotherapy and Oncology. 2016.

23. Yip SS, Kim J, Coroller T, Parmar C, Rios Velazquez E, Huynh E, Mak R, Aerts HJ. Associations between somatic mutations and metabolic imaging phenotypes in non-small cell lung cancer. Journal of Nuclear Medicine. 2016.

24. Huang Y, Liu Z, He L, Chen X, Pan D, Ma Z, Liang C, Tian J, Liang C. Radiomics Signature: A Potential Biomarker for the Prediction of Disease-Free Survival in Early-Stage (I or II) Non-Small Cell Lung Cancer. Radiology. 2016:152234.

25. Desseroit M-C, Tixier F, Weber WA, Siegel BA, Cheze Le Rest C, Visvikis D, Hatt M. Reliability of PET/CT shape and heterogeneity features in functional and morphological components of Non-Small Cell Lung Cancer tumors: a repeatability analysis in a prospective multi-center cohort. Journal of Nuclear Medicine: Official Publication, Society of Nuclear Medicine. 2016.

26. Bayanati H, E. Thornhill R, Souza CA, Sethi-Virmani V, Gupta A, Maziak D, Amjadi K, Dennie C. Quantitative $C T$ texture and shape analysis: Can it differentiate benign and malignant mediastinal lymph nodes in patients with primary lung cancer? European Radiology. 2015;25(2):480-487.

27. Andersen MB, Harders SW, Ganeshan B, Thygesen J, Torp Madsen HH, Rasmussen F. CT texture analysis can help differentiate between malignant and benign lymph nodes in the mediastinum in patients suspected for lung cancer. Acta Radiologica. 2015.

28. Li H, Becker N, Raman S, Chan TCY, Bissonnette J-P. The value of nodal information in predicting lung cancer relapse using 4DPET/4DCT. Medical Physics. 2015;42(8):4727-4733.

29. Betticher DC. Mediastinal Lymph Node Clearance After Docetaxel-Cisplatin Neoadjuvant Chemotherapy Is Prognostic of Survival in Patients With Stage IIIA pN2 Non-Small-Cell Lung Cancer: A Multicenter Phase II Trial. Journal of Clinical Oncology. 2003;21(9):1752-1759.

30. Jaklitsch MT, Herndon JE, DeCamp MM, Richards WG, Kumar P, Krasna MJ, Green MR, Sugarbaker DJ. Nodal downstaging predicts survival following induction chemotherapy for stage IIIA (N2) non-small cell lung cancer in CALGB protocol \#8935. Journal of Surgical Oncology. 2006;94(7):599-606.

31. Suntharalingam M, Paulus R, Edelman MJ, Krasna M, Burrows W, Gore E, Wilson LD, Choy H. Radiation therapy oncology group protocol 02-29: a phase II trial of neoadjuvant therapy with concurrent chemotherapy and full-dose radiation therapy followed by surgical resection and consolidative therapy for locally advanced non-small cell carcinoma of the lung. International Journal of Radiation Oncology, Biology, Physics. 2012;84(2):456-463. 
32. National Comprehensive Cancer Network. NCCN guidelines: Non-Small Cell Lung Cancer (Version 7.2015). 2015.

33. Pieper S, Halle M, Kikinis R. 3D Slicer. In: IEEE International Symposium on Biomedical Imaging: Nano to Macro, 2004.; 2004:632-635 Vol. 1.

34. Anon. $R$ Core Team (2013). R: A language and environment for statistical computing. $R$ Foundation for Statistical Computing, Vienna, Austria. ISBN 3-900051-07-0, URL http://www.R-project.org/.

35. Schröder MS, Culhane AC, Quackenbush J, Haibe-Kains B. survcomp: an R/Bioconductor package for performance assessment and comparison of survival models. Bioinformatics. 2011;27(22):3206-3208.

36. Benjamini Y, Hochberg Y. Controlling the False Discovery Rate: A Practical and Powerful Approach to Multiple Testing. Journal of the Royal Statistical Society. Series B (Methodological). 1995;57(1):289-300.

37. Liaw A, Wiener M. Classification and Regression by randomForest. R News. 2002;2(3):18-22.

38. Kuhn M. Building predictive models in $R$ using the caret package. Journal of Statistical Software. 2008;28(5):1-26.

39. Zhao B, James LP, Moskowitz CS, Guo P, Ginsberg MS, Lefkowitz RA, Qin Y, Riely GJ, Kris MG, Schwartz LH. Evaluating Variability in Tumor Measurements from Same-day Repeat CT Scans of Patients with NonSmall Cell Lung Cancer 1. Radiology. 2009;252(1):263-272. 



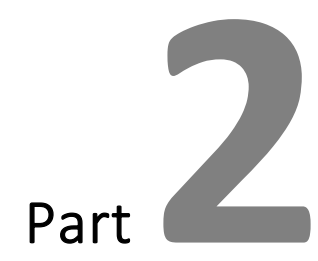

\section{Lung Tumor Evolution After Radiotherapy Treatment}





\section{Chapter}

\section{CT-based radiomic signature predicts distant metastasis in lung adenocarcinoma}

Published in: Radiotherapy and Oncology (2015)

CT-based radiomic signature predicts distant metastasis in lung adenocarcinoma.

Thibaud Coroller*, Patrick Grossmann*, Ying Hou, Emmanuel Rios Velazquez, Ralph Leijenaar., Gretchen Hermann, Philippe Lambin, Benjamin Haibe-Kains, Raymond Mak*, Hugo Aerts* 


\section{ABSTRACT}

Background and Purpose: Radiomics provides opportunities to quantify the tumor phenotype non-invasively by applying a large number of quantitative imaging features. This study evaluates computed-tomography (CT) radiomic features for their capability to predict distant metastasis (DM) for lung adenocarcinoma patients.

Material and Methods: We included two datasets: 98 patients for discovery and 84 for validation. The phenotype of the primary tumor was quantified on pre-treatment CTscans using 635 radiomic features. Univariate and multivariate analysis was performed to evaluate radiomics performance using the concordance index $(\mathrm{Cl})$.

Results: Thirty-five radiomic features were found to be prognostic $(\mathrm{Cl}>0.60, \mathrm{FDR}<5 \%)$ for DM and twelve for survival. It is noteworthy that tumor volume was only moderately prognostic for $\mathrm{DM}\left(\mathrm{Cl}=0.55, \mathrm{p}\right.$-value $\left.=2.77 \times 10^{-5}\right)$ in the discovery cohort. A radiomicsignature had strong power for predicting DM in the independent validation dataset $\left(\mathrm{Cl}=0.61, \mathrm{p}\right.$-value $\left.=1.79 \times 10^{-17}\right)$. Adding this radiomic-signature to a clinical model resulted in a significant improvement of predicting $D M$ in the validation dataset ( $p$ value $=1.56 \times 10^{-11}$ ).

Conclusions: Although only basic metrics are routinely quantified, this study shows that radiomic features capturing detailed information of the tumor phenotype can be used as a prognostic biomarker for clinically-relevant factors such as DM. Moreover, the radiomic-signature provided additional information to clinical data. 


\section{INTRODUCTION}

Lung cancer is the most deadly cancer worldwide for both men and women ${ }^{1}$. Non-small cell lung cancer (NSCLC) is the most common type of lung cancer (85-90\% of all lung cancers) and adenocarcinoma is the most common subtype (about $40 \%$ of all lung cancers) of NSCLC. Patients with locally advanced (stage II-III) lung adenocarcinomas are typically treated with combined modality therapy including chemotherapy with local therapy including radiation therapy and/or surgery, but overall survival remains low due to a high risk of local recurrence and distant metastasis (DM) after treatment. Despite the use of concurrent chemotherapy with local therapy, the incidence of DM after combined modality therapy is as high as $30-40 \%$ in prospective trials ${ }^{2-4}$. However, large randomized trials studying consolidation chemotherapy after concurrent chemotherapy and radiation therapy have not shown improvement in overall survival with additional chemotherapy ${ }^{5,6}$ likely because there was no selection of patients at the highest risk of DM. Therefore, developing better biomarkers to predict patients at highest risk for DM may help identify sub-groups who benefit from intensification of systemic therapy and is crucial for improving outcomes.

Due to recent technological advances in medical imaging it is possible to capture tumor phenotypic characteristics non-invasively. The most widely used imaging modality is Computed-Tomography (CT), which can quantify tissue density. In lung cancer, CT imaging is routinely used for patient management, including diagnosis, radiation treatment planning and surveillance.

Tumour phenotypic differences (e.g. shapes irregularity, infiltration, heterogeneity or necrosis) can be quantified in CT images using radiomic features. Radiomics ${ }^{7-9}$ aims to provide a comprehensive quantification of the tumor phenotype by analyzing robustly 10-12 a large set of quantitative data characterization algorithms. Biomarkers based on quantitative features have demonstrated strong prognostic performance across a range of cancer types and investigators have reported that these features are associated with clinical outcomes and underlying genomic patterns ${ }^{13-26}$. Radiomics has significant clinical potential, as it can be applied to routinely acquired medical imaging data at low costs.

In this manuscript we present a radiomic analysis to identify biomarkers of DM in patients treated with chemoradiation (chemoRT) for locally advanced lung adenocarcinoma. In a discovery dataset, we extracted 635 radiomics features to identify the optimal features for predicting metastasis. Only a limited number of features with high performance for predicting DM were tested in the independent validation dataset. We evaluated the ability of radiomic features to predict DM or overall survival, and how these features compare with basic metrics (e.g. volume, diameter) as prognostic factors ${ }^{27-30}$. 


\section{MATERIALS AND METHODS}

Patient characteristics. This study is an Institutional Review Board-approved analysis of CT for treatment simulation from North-American NSCLC patients receiving chemoRT at our institution from 2001 to 2013. We limited the patient population to pathologicallyconfirmed lung adenocarcinoma with locally advanced disease (overall stage II-III) ${ }^{30}$. Patients with surgery or chemotherapy before the scheduled radiation therapy planning CT date were excluded from the study. Patients treated before July 2009 were included in the discovery Dataset1 ( $n=98)$, and after July 2009 in an independent validation Dataset2 $(n=84)$. In total 182 patients were included in our analysis.

Clinical endpoints. Patients were followed up every three to six months after treatment, and surveillance chest CT scans with contrast (unless patient's contraindication, e.g. allergy or renal dysfunction) were performed to assess treatment response or tumor progression based on US national guidelines (NCCN). The primary endpoint of this study was distant metastasis (DM), which was defined as progression of disease to other organs as assessed in surveillance scans, and time to DM was defined as time from start of radiation to date of DM or censoring (date of last scan). Overall survival was analyzed as a secondary endpoint, and was defined as the time between the start of radiation treatment and last day of follow up or date of death.

Clinical variables. The conventional clinical prognostic factors (CPFs) used for this study included tumor grade (1-Well differentiated, 2-Moderately differentiated, 3-Poorly differentiated and 4-Not available), Eastern Cooperative Oncology Group (ECOG) performance status (PS) ${ }^{31}$, TNM stage per the American Joint Committee on Cancer (AJCC) staging system $\left(7^{\text {th }} \text { edition }\right)^{30} ;$ CT-based measurements commonly utilized in the clinic (e.g. tumor volume and maximal tumor diameter measured on single axial slice ), and treatment characteristics. Sub-group analyses of clinical variables were performed (e.g. overall stage II vs IIIA vs IIIB) and can be found in Table S1 (Supplement II.1).

CT acquisition and segmentation. Planning CT was performed according to standard clinical scanning protocols at our institution with a GE "LightSpeed" CT scanner (GE Medical System, Milwaukee, WI, USA). The most common pixel spacing was $(0.93 \mathrm{~mm}$, $0.93 \mathrm{~mm}, 2.5 \mathrm{~mm}$ ) for CT. The primary lung tumor was delineated manually on Eclipse (Varian Medical System, Palo Alto, CA, USA). It was first contoured in the abdomen window to identify the boundaries with the chest wall or other soft tissues, then in the lung window to capture the maximum extent in the lung parenchyma. All contours were reviewed by an experienced radiation oncologist (R.H.M).

Radiomic features extraction. Radiomic features have the capacity to capture tumor phenotype differences by examining a large set of quantitative features (Figure 1). The feature extraction was performed in MATLAB 2013b (Mathworks, Natick, MA, USA) using an in-house developed toolbox running on the Computational Environment for 
Radiotherapy Research (CERR) ${ }^{32}$. DICOMs files (CT images + tumor contours) were imported into CERR to extract the radiomic features. The radiomic features set included is described in detail in the Supplement I.
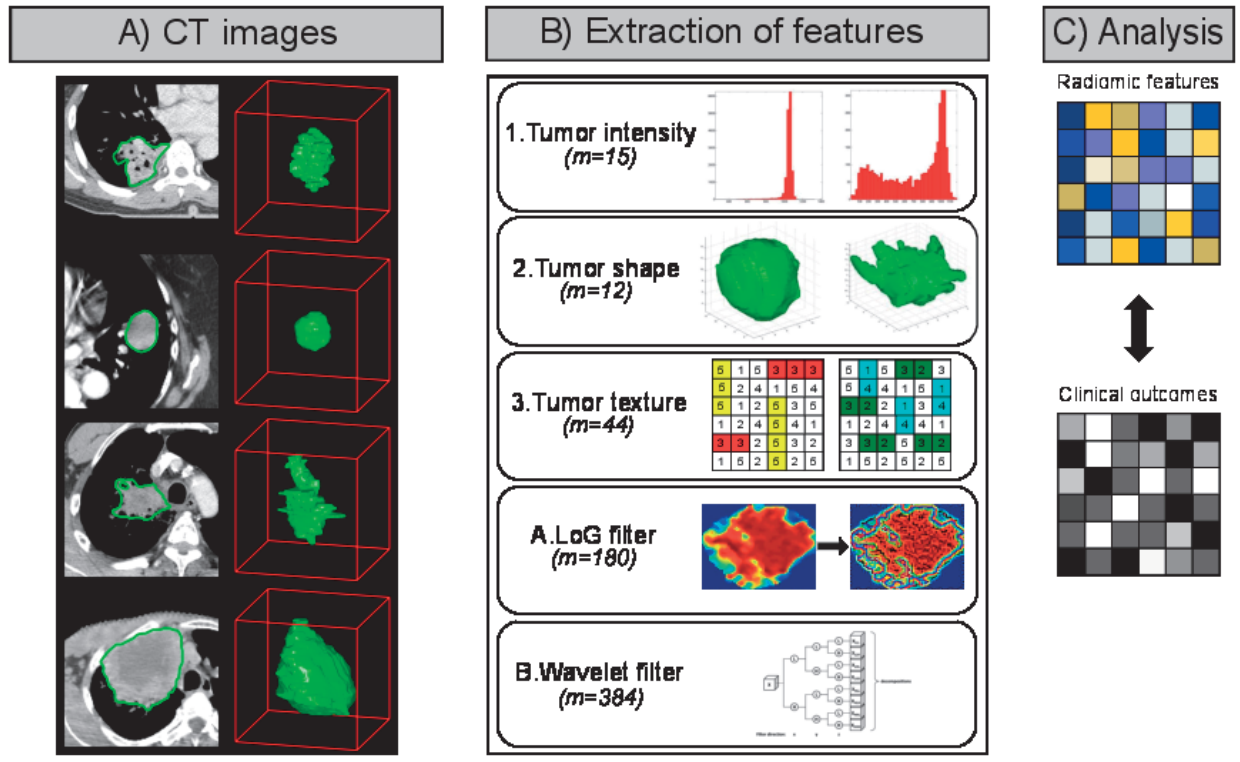

Figure 5. A) Differences between lung primary tumors with a same histology are apparent on CT images (3D model on the right and CT contours on the left). CT images of primary tumors contain critical information that can be used to predict outcomes or assess the RT treatment response. B) To quantify this information, a large set of features $(m=635)$ is used to capture the tumor phenotype. It includes 1 | intensity, 2 | shape and 3 | texture based features. Also, A| Laplacian of Gaussian (LoG) and B| Wavelet filtered features were investigated. C) The final step is to link radiomic information to clinical data.

Feature selection. Feature selection for the radiomic signature was performed with the minimum redundancy maximum relevance (mRMR) algorithm implemented in the mRMRe ${ }^{33}$ package version 2.0.4 in R. The mRMR algorithm is an entropy based feature selection method, which starts by calculating the mutual information (MI) between a set of features and an outcome variable. MRMR ranks the input features by maximizing the $\mathrm{MI}$ with respect to outcome and minimizing the average $\mathrm{Ml}$ of higher ranked features. Here, survival objects as implemented in R with "Survcomp" package ${ }^{34}$ were used as outcome to select complementary features with respect to DM or survival.

Among available clinical covariates, those with $p<0.1$ on univariate analysis of DM using a Log-Rank test were included into a multivariate clinical prognostic model. 


\section{Data analysis}

Univariate and multivariate analyses were performed for this study. All analysis were performed on Dataset1, leaving Dataset2 as an independent validation cohort for evaluating the radiomic signature.

Statistical analysis was conducted using the survcomp ${ }^{34}$ package version 1.12 and rme$\mathrm{ta}^{35}$ package version 2.16 in Bioconductor ${ }^{36}$. Prognostic performances were evaluated by the concordance index ${ }^{37}(\mathrm{Cl})$, which is the probability that among two randomly drawn samples, the sample with the higher risk value has also the higher chance of experiencing an event (e.g. death or development of DM). Cls were either directly computed for continuous variables or on the predictions of a univariate Cox model with clinical categorical variables. Kaplan-Meier and Log-Rank statistics were used to analyze the univariate discrimination of survival and DM groups by imaging features and clinical covariates. To build the multivariate radiomic signature for DM, Cox regression models were trained on Dataset1 for selected prognostic variables and the predictions by these models were validated on Dataset2. Features were incrementally added to the model according to the relevance rank calculated by $\mathrm{mRMR}^{33}$. Intermediate models were tested by repeated random sub-sampling cross validation with 1,000 iterations on Dataset1. Once the mean $\mathrm{Cl}$ of the growing model dropped, the corresponding feature set was retained selected as the final model. Only this selected model was and validated on Dataset2. Significance of Cls was assessed by bootstrapping subsamples of size 100 with 100 repetitions for $A$ ) true survival data and $B$ ) random permutations of survival data, and comparing the empirical distributions of A) and B) by an one-sided Wilcoxon signed rank test. The same procedure was used to assess if a $\mathrm{Cl}$ was higher than another $\mathrm{Cl}$. To correct for multiple comparisons, we additionally adjusted P-values by the falsediscovery-rate (FDR) procedure according to Benjamini and Hochberg ${ }^{38}$. All statistical analysis was performed using the R software ${ }^{39}$ version 3.0.2.

\section{RESULTS}

The majority of all patients were female (62.6\%) and the median age at start of treatment was 64 years (range: 35-93 years). The median follow-up time was 23.7 months (range: 1.8-119.2 months) and the median survival time was 24.7 months (range: 1.8119.2 months). The median time to distant metastasis (DM) was 13.4 months (range: 0.3-117.5 months). Patient characteristics, clinical outcomes are shown in Table 1.

Time to DM was similar between Dataset1 and Dataset2 ( $p$-value $<0.36$ ), as for the numbers of DM ( $p$-value $<0.45)$. However, survival ( $p$-value $<0.005$ ) and follow-up times ( $p$-value $<0.007)$ were significantly different in Dataset1. 
Table 2: Patient characteristics and outcomes are reported for each datasets.

\begin{tabular}{|c|c|c|c|c|}
\hline & $\begin{array}{l}\text { Overall dataset } \\
(n=182)\end{array}$ & Dataset $1(n=98)$ & Dataset $2(n=84)$ & P-value \\
\hline & Median (range) & Median (range) & Median (range) & \\
\hline Age [years] & $64(35-93)$ & $62(41-86)$ & $65(35-93)$ & 0.63 \\
\hline Gender $[\mathrm{F} / \mathrm{M}]$ & $\begin{array}{l}114(62.6 \%) / \\
68(37.4 \%)\end{array}$ & $\begin{array}{l}66(67.3 \%) / \\
32(32.7 \%)\end{array}$ & $\begin{array}{l}48(57.1 \%) / \\
36(42.9 \%)\end{array}$ & 0.29 \\
\hline Overall stage [IIA/IIB/IIIA/IIIB] & $6 / 3 / 101 / 72$ & $2 / 1 / 55 / 40$ & $4 / 2 / 46 / 32$ & 0.65 \\
\hline T-stage [T1a/T1b/T2a/T2b/T3/T4] & $19 / 23 / 50 / 19 / 39 / 32$ & $14 / 10 / 30 / 10 / 17 / 17$ & $5 / 13 / 20 / 9 / 22 / 15$ & 0.26 \\
\hline $\mathrm{N}$-stage [N0/N1/N2/N3] & $13 / 17 / 97 / 55$ & $5 / 9 / 53 / 31$ & $8 / 8 / 44 / 24$ & 0.70 \\
\hline Performance status [0/1/2/3] & $81 / 91 / 8 / 2$ & $36 / 57 / 5 / 0$ & $45 / 34 / 3 / 2$ & 0.04 \\
\hline Tumor grade $[1 / 2 / 3 / X]$ & $4 / 28 / 92 / 58$ & $3 / 11 / 47 / 37$ & $1 / 17 / 45 / 21$ & 0.12 \\
\hline Follow-up [months] & $23.7(1.8-119.2)$ & $28.9(1.8-119.2)$ & $19.5(3.1-54.9)$ & 0.007 \\
\hline Survival [months] & $24.7(1.8-119.2)$ & $29.7(1.8-119.2)$ & $21.4(3.4-54.9)$ & 0.005 \\
\hline Time to distant metastasis [months] & $13.4(0.3-117.5)$ & $13.6(0.3-117.5)$ & $13.3(0.7-49.6)$ & 0.36 \\
\hline Distant metastasis [No/Yes] & $\begin{array}{l}69(37.9 \%) / \\
113(62.1 \%)\end{array}$ & $\begin{array}{l}34(34.7 \%) / \\
64(65.3 \%)\end{array}$ & $\begin{array}{l}3541.7 \%) / \\
49(58.3 \%)\end{array}$ & 0.45 \\
\hline $\begin{array}{l}\text { Radiation dose delivered } \\
\leq 54 / \leq 60 / \leq 66 />66 \text { [Gray] }\end{array}$ & $\begin{array}{l}60(32.97 \%) / \\
30(16.48 \%) / \\
70(38.45 \%) / \\
22(12.1 \%)\end{array}$ & $\begin{array}{l}28(28.57 \%) / \\
17(17.35 \%) / \\
33(33.67 \%) / \\
20(20.41 \%)\end{array}$ & $\begin{array}{l}32(38.10 \%) / \\
13(15.48 \%) / \\
37(44.04 \%) / \\
2(2.38 \%)\end{array}$ & 0.002 \\
\hline $\begin{array}{l}\text { Chemotherapy sequence } \\
\text { [concurrent/adjuvant/induction] }\end{array}$ & $175 / 79 / 28$ & $95 / 38 / 22$ & $80 / 41 / 6$ & 0.024 \\
\hline
\end{tabular}

For categorical variables, actual numbers are reported for each category (format A/B/C). Statistical comparison between dataset 1 and 2 was computed using Chi Square (categorical variables) or Wilcoxon rank sum test (continuous variables).

We investigated the association of radiomics data with DM and overall survival. In Figure 2 the association of the imaging features with DM and survival in the discovery Dataset1 is shown. Of the complete radiomic features set $(m=635)$, a total of $520(81.88 \%)$ and 582 (91.65\%) features were significant from random (FDR < 5\%) for DM and survival, respectively. A total of 445 radiomic features were significant for both DM and survival. A high linear relationship was observed $\left(R^{2}=0.92\right.$, $p$-value $\left.<10^{-243}\right)$, for the features significant for both DM and survival. It is noteworthy that LoG features had the highest performance compared to the other features groups. 


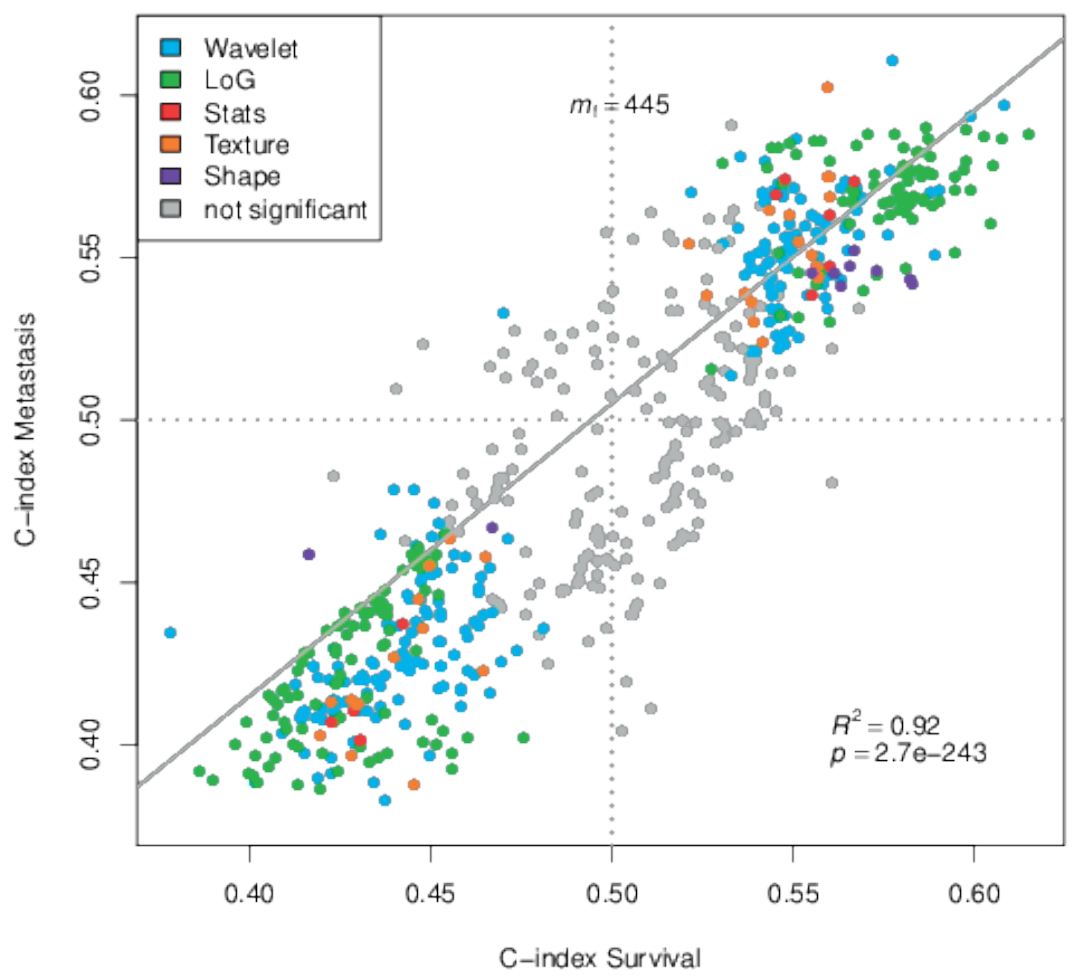

Figure 2. Univariate performances of prognostic features for Distant Metastasis (DM) and survival. Each point refers to the $\mathrm{Cl}$ of a feature evaluating the power of feature to predict metastasis, respectively, survival. Colors refer to the type of feature. Features whose $\mathrm{Cl}$ estimation was not significant (FDR $<5 \%$ ) for both DM and survival are shown in grey. Overall, 445 of these pairs of $\mathrm{Cls}$ are considered to be significant estimates. Linear regression for all significant pairs of Cls yielded an R-squared value of 0.92 (F-test, p-value 2.5e-243).

Among all features, thirty-five radiomics features were strongly prognostic $(\mathrm{Cl}>0.60$ and FDR < 5\%) for DM (Table S2 in the Supplement II.5). Twelve features were found prognostic for survival. Specific details on statistic values of these features can be found in Table S3 in Supplement II.5. Between these two top performing feature sets there were four common prognostic features for both DM and survival. All of them were LoG based features (3 entropy and 1 standard deviation).

We compared the top 15 features that had the highest Cls (Top15), with tumor volume and diameter (equivalent to basic metrics). The Top15 radiomic features had notably higher Cls compared to tumor volume and diameter (Figure 3.A).

We also investigated the association of CPFs with DM in our data set. Three clinical parameters appeared to be significant univariate prognostic factors: Overall Stage $\left(\mathrm{Cl}=0.63\right.$, $\mathrm{p}$-value $\left.<6.78 \times 10^{-14}\right)$, Gender $\left(\mathrm{Cl}=0.63, \mathrm{p}\right.$-value $\left.<2.35 \times 10^{-11}\right)$ and tumor grade $\left(\mathrm{Cl}=0.61, \mathrm{p}\right.$-value $\left.<2.35 \times 10^{-11}\right)$. Clinical parameters, ranked by their $\mathrm{Cl}$ are displayed in 
Figure 3.B. Overall stage and gender yielded a higher $\mathrm{Cl}$ than the radiomic features, although their $95 \%$ confidence interval is wider compared to the radiomic features.

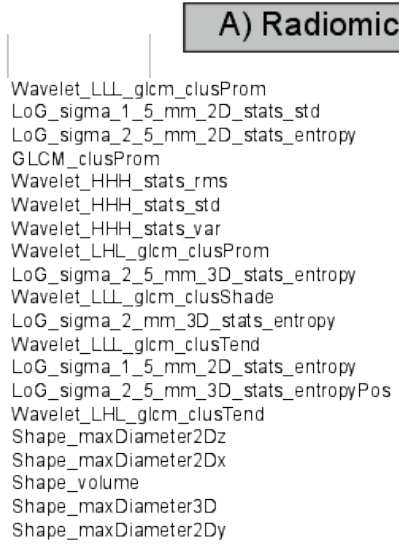

\section{A) Radiomic features}

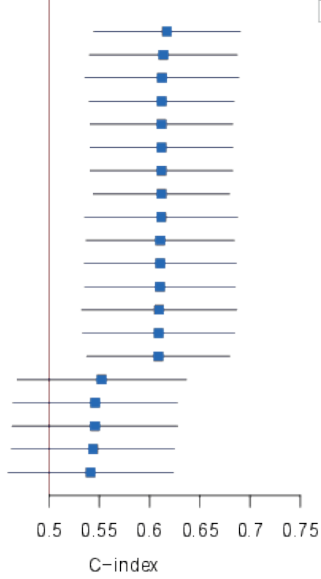

Figure 3. A) Forest plot of the 15 best performing radiomic features for Distant Metastasis on univariate analysis (Dataset1, $n=98$ ). Radiomics equivalent of basic metrics (diameter and volume) were added for comparison. B) Forest plot of the clinical factors. The absolute C-indices and their $95 \%$ confidence interval are shown.

An mRMR based feature selection on all features on Dataset1 $(n=98)$ was performed to reduce redundancy and select a potential set of complementary and prognostic features. From this new ranking, the 15 highest mRMR-ranked features were kept after feature selection to build the radiomic signature. A multivariate Cox regression model to predict DM was developed. Features were iteratively added in order of high to low mRMR rank on Dataset1, and Dataset2 was used for independent validation. The combination that yielded the maximum $\mathrm{Cl}$ on the discovery Dataset1 before dropping was defined as the optimal radiomic signature for predicting DM. This signature consists of three features: 1) Wavelet HHL - Skewness, 2) Gray-Level Co-occurrence Matrix - Cluster shade, and 3) LoG 5mm 2D - Skewness. Cluster shade is a textural feature sensitive to tumor heterogeneities. Skewness is a first-order feature that measures the asymmetry of the histogram from the mean, which here is associated with two different filters LoG and Wavelet.

As a final step, we compared the radiomic signature to a clinical Cox regression model containing covariates that significantly discriminated between patients with and without DM in Dataset1 in univariate analysis. The final model contained overall stage and tumor grade. This clinical model showed moderate prognostic power when applied to Dataset2 with coefficients trained on Dataset1 $\left(\mathrm{Cl}=0.57\right.$, $\mathrm{p}$-value $\left.<1.03 \times 10^{-7}\right)$. Combining the clinical and radiomic signature (trained on Dataset1) showed a significantly ( $p$-value $\left.<1.56 \times 10^{-11}\right)$ higher association with DM when applied to Dataset2 $(\mathrm{Cl}=0.60, \mathrm{p}$-value $<$ 
$\left.3.57 \times 10^{-16}\right)$, compared to the clinical model. A median split of the patient prediction scores from applying the combined model on Dataset2 yielded a significant difference $(p$-value $=0.049)$ for metastasis-free probability estimates (Figure 4$)$.

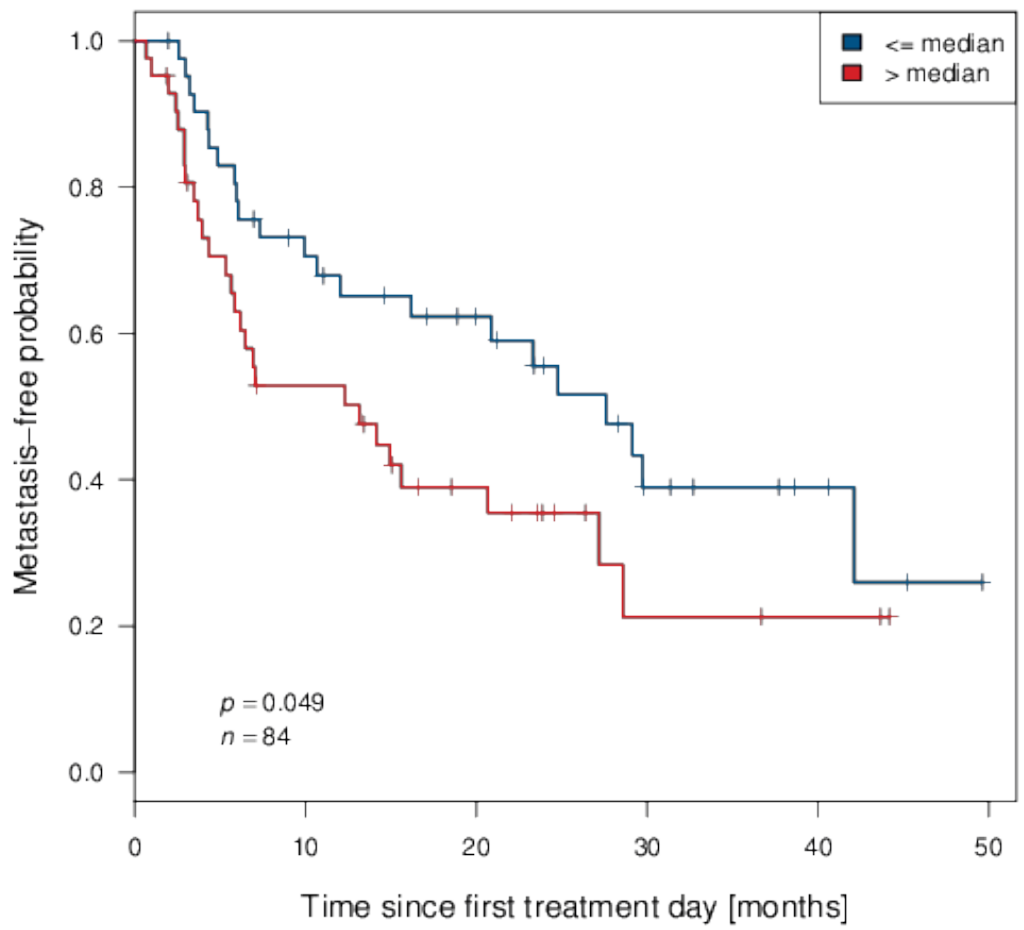

Figure 4. Kaplan Meier curves according to the combined model predicting score to predict metastasis-free probability in an independent dataset. A significant survival difference appears between patients with a high or low risk of Distant Metastasis (Dataset2, $n=84$, Log-Rank test, $p$-value $<0.049$ ).

\section{DISCUSSION}

Medical imaging gives valuable information for diagnostic, treatment planning or surveillance of cancer patients. Routinely, basic metrics are extracted from these images to utilize as a prognostic factor ${ }^{27-30}$, or to assess treatment response. However, there is much more tumor phenotypic information captured in these images. Radiomics are able to quantify tumor phenotypical differences from medical images by using a large set of imaging features that can be linked to clinical factors of the tumors. In this study we extracted 635 radiomic features from a total of 182 lung cancer patients treated with chemoRT to assess the ability of radiomic features as a prognostic biomarker for distant metastasis (DM), and we validated a radiomic-based signature on an independent validation dataset. Since DM remains a major cause of mortality in $30-40 \%$ of patients with locally advanced lung adenocarcinoma, early identification of patients at highest risk of 
developing DM would allow clinicians to adapt treatment such as incorporating consolidation chemotherapy to improve outcomes. Moreover, the theoretical benefit of consolidation chemotherapy has not been shown in large randomized studies to date. It is likely because there was no selection of patients at the highest risk of distant metastases (i.e. patients who were at low risk of distant metastases were included in these trials and would not need additional treatment). Future trial design to demonstrate benefits of consolidation chemotherapy will likely require stratification to identify those at highest risk of distant metastases and may benefit most from additional treatment.

We observed strong individual correlations between clinical outcomes and quantitative imaging features. A large number of features were significant from random to predict DM (91\%) and survival (82\%) in univariate analysis after correction for multiple testing. Moreover, a high linear correlation was found among those 445 features that were significant factors of both DM and survival $\left(R^{2}=0.92, \mathrm{p}\right.$-value $\left.<10^{-243}\right)$. This high linear correlation is expected as there is a high correlation between DM and survival (DM greatly impact patient survival, see Table S4). Only a small number of features, 35 for DM and for 12 survival, were prognostic, as defined by $\mathrm{Cl}>0.6$ and $\mathrm{FDR}<5 \%$.

Although we tested a large number of features, to minimize any risk of over-fitting or bias, we performed a robust validation approach: all analysis steps, mRMR feature selection, and model fitting were performed on Dataset1 $(n=98)$ and the results validated on an independent validation Dataset2 $(n=84)$. With this approach we found a multivariate radiomic DM signature consisting of three features that yielded a high prognostic performance for DM in Dataset1 $(\mathrm{Cl}=0.61)$. Combining the radiomic signature to a clinical predictors showed significant improvement ( $p$-value $\left.<1.56 \times 10^{-11}\right)$, compared to the clinical predictors alone.

A recent study from Fried et $a .^{22}$ investigated DM prediction for NSCLC patients. They found a significant model DM (P-value $=0.005)$ using both texture features and CPFs. The model used consisted of eight parameters (two CPFs and six textures). In another study, Ganeshan et al. ${ }^{15}$ applied textural analysis to find univariate prognostic factors for survival. They focused on two imaging features (uniformity, associated with two LoG filter). In our analysis, these features were significant from random but lowly ranked by their $\mathrm{Cl}$ value $\left(184^{\text {th }}\right.$ and $146^{\text {th }} \mathrm{Cl}$-ranked features in Dataset1). However, major differences in studies design and implementation made it difficult to compare them objectively. Fields et $a{ }^{22}$ used leave-one out cross validation to validate their model instead of an independent validation dataset. Ganeshan et $a . .^{15}$ only used one CT image slice (presenting the largest cross section) to calculate their features when we used the whole primary tumor. Finally, both these studies have a smaller patient cohort, $n=54$ [7] and $n=91^{22}$, and had mixed histology patients. Our analysis calculated the features from the complete 3D tumor volume, contained only a single histology of NSCLC (adenocarcinoma), and is based on larger cohorts ( $n=182$ ) with an independent validation dataset for the radiomic signature. 
A complementary point of the study was to compare basic metrics ${ }^{27-29}$ to radiomic features as prognostic factors for DM. The first observation made was that ShapeMaximum diameter (in every direction $\mathrm{x} / \mathrm{y} / \mathrm{z}$ ) is a better univariate prognostic factors than the maximal tumor diameter on an axial slice reported by a radiologist. The advantage of the radiomic shape features is that they can be automatically acquired, reproducible $\mathrm{e}^{10-12}$, and take into account the whole tumor volume, whereas clinically assessed tumor diameters are manually drawn on a CT slice and are therefore limited to one dimension of the tumor. Furthermore, shape or size-based features were not in the top ranked features in our study. Total tumor volume, has been associated with survival in stage I-III NSCLC patients treated with radiation therapy in a study from Etiz et al. ${ }^{28}$, and a prior study from our institution by Alexander et $a .^{29}$ also demonstrated an association between primary tumor volume and overal survival, but not risk of distant metastasis. In our study, volume was ranked only the $405^{\text {th }}(\mathrm{Cl}=0.55)$ and $224^{\text {th }}(\mathrm{Cl}=0.56)$ best univariate prognostic factor for DM and survival respectively in Dataset1. Thus, while basic metrics such as size and volume have historically been used as used in the clinical setting because such data is easily acquired, radiomic shape and size measurements can provide stronger prognostic factors.

A short-coming of our study is the variability in CT acquisition and reconstruction parameters. Our dataset includes patients from 2001 to 2013. During this time period, the standard of care for CT acquisition has evolved, differences appeared between our cohorts for some factors (Table1). However, despite this variability in the imaging data (evolution of hardware, progress in informatics), radiomics was able to detect a strong signal to predict DM despite a temporal split. Additionally, clinical outcomes are provided by one center, which makes it hard to evaluate the generalizability of outcomes to other institutions. However, in comparison to a recent study ${ }^{20}$ investigating clinical outcomes from another center, patient characteristics or outcomes were comparable. Future work would therefore involve studying the DM signature in other histologies and in independent validation sets from other institutions, assessing its generalizability to all NSCLC.

In conclusion, this study demonstrated strong association between radiomic features and DM for patients with locally advanced adenocarcinoma; and presented an independently validated radiomics signature for DM. This signature would allow early identification of patients with locally advanced lung adenocarcinoma at risk of developing DM, allowing clinicians to individualize treatment (such as intensification of chemotherapy) to reduce the risk of DM and improve survival.

\section{ACKNOWLEDGMENTS}

We acknowledge financial support from the National Cancer Institute (NIH). 


\section{REFERENCES}

1. Siegel R, Ma J, Zou Z, Jemal A. Cancer statistics, 2014: Cancer Statistics, 2014. CA: A Cancer Journal for Clinicians. 2014;64(1):9-29.

2. Albain KS, Swann RS, Rusch VW, Turrisi AT, Shepherd FA, Smith C, Chen Y, Livingston RB, Feins RH, Gandara DR, Fry WA, Darling G, Johnson DH, Green MR, Miller RC, et al. Radiotherapy plus chemotherapy with or without surgical resection for stage III non-small-cell lung cancer: a phase III randomised controlled trial. Lancet. 2009;374(9687):379-386.

3. Curran WJ, Paulus R, Langer CJ, Komaki R, Lee JS, Hauser S, Movsas B, Wasserman T, Rosenthal SA, Gore E, Machtay M, Sause W, Cox JD. Sequential vs. concurrent chemoradiation for stage III non-small cell lung cancer: randomized phase III trial RTOG 9410. Journal of the National Cancer Institute. 2011;103(19):1452-1460.

4. Bradley JD, Paulus R, Komaki R, Masters GA, Forster K, Schild SE, Bogart J, Garces YI, Narayan S, Kavadi V, Nedzi LA, Michalski JM, Johnson D, MacRae RM, Curran WJ, et al. A randomized phase III comparison of standard-dose (60 Gy) versus high-dose (74 Gy) conformal chemoradiotherapy with or without cetuximab for stage III non-small cell lung cancer: Results on radiation dose in RTOG 0617. ASCO Meeting Abstracts. 2013;31(15_suppl):7501.

5. Hanna N, Neubauer M, Yiannoutsos C, McGarry R, Arseneau J, Ansari R, Reynolds C, Govindan R, Melnyk A, Fisher W, Richards D, Bruetman D, Anderson T, Chowhan N, Nattam S, et al. Phase III Study of Cisplatin, Etoposide, and Concurrent Chest Radiation With or Without Consolidation Docetaxel in Patients With Inoperable Stage III Non-Small-Cell Lung Cancer: The Hoosier Oncology Group and U.S. Oncology. Journal of Clinical Oncology. 2008;26(35):5755-5760.

6. Kelly K, Chansky K, Gaspar LE, Albain KS, Jett J, Ung YC, Lau DHM, Crowley JJ, Gandara DR. Phase III trial of maintenance gefitinib or placebo after concurrent chemoradiotherapy and docetaxel consolidation in inoperable stage III non-small-cell lung cancer: SWOG S0023. Journal of Clinical Oncology: Official Journal of the American Society of Clinical Oncology. 2008;26(15):2450-2456.

7. Lambin P, Rios-Velazquez E, Leijenaar R, Carvalho S, van Stiphout RGPM, Granton P, Zegers CML, Gillies R, Boellard R, Dekker A, Aerts HJWL. Radiomics: Extracting more information from medical images using advanced feature analysis. European Journal of Cancer. 2012;48(4):441-446.

8. Kumar V, Gu Y, Basu S, Berglund A, Eschrich SA, Schabath MB, Forster K, Aerts HJWL, Dekker A, Fenstermacher D, Goldgof DB, Hall LO, Lambin P, Balagurunathan Y, Gatenby RA, et al. Radiomics: the process and the challenges. Magnetic Resonance Imaging. 2012;30(9):1234-1248.

9. Lambin P, van Stiphout RGPM, Starmans MHW, Rios-Velazquez E, Nalbantov G, Aerts HJWL, Roelofs E, van Elmpt W, Boutros PC, Granone P, Valentini V, Begg AC, De Ruysscher D, Dekker A. Predicting outcomes in radiation oncology--multifactorial decision support systems. Nature reviews. Clinical oncology. 2013;10(1):27-40.

10. Rios Velazquez E, Aerts HJWL, Gu Y, Goldgof DB, De Ruysscher D, Dekker A, Korn R, Gillies RJ, Lambin P. A semiautomatic CT-based ensemble segmentation of lung tumors: Comparison with oncologists' delineations and with the surgical specimen. Radiotherapy and Oncology. 2012;105(2):167-173.

11. Parmar C, Rios Velazquez E, Leijenaar R, Jermoumi M, Carvalho S, Mak RH, Mitra S, Shankar BU, Kikinis R, Haibe-Kains B, Lambin P, Aerts HJWL. Robust Radiomics Feature Quantification Using Semiautomatic Volumetric Segmentation. Woloschak GE, ed. PLOS ONE. 2014;9(7):e102107.

12. Leijenaar RTH, Carvalho S, Velazquez ER, van Elmpt WJC, Parmar C, Hoekstra OS, Hoekstra CJ, Boellaard R, Dekker ALAJ, Gillies RJ, Aerts HJWL, Lambin P. Stability of FDG-PET Radiomics features: An integrated analysis of test-retest and inter-observer variability. Acta Oncologica. 2013;52(7):1391-1397.

13. Ganeshan B. Non_Small Cell Lung Cancer: Histopathologic Correlates for Texture Parameters at CT.

14. Davnall F, Yip CSP, Ljungqvist G, Selmi M, Ng F, Sanghera B, Ganeshan B, Miles KA, Cook GJ, Goh V. Assessment of tumor heterogeneity: an emerging imaging tool for clinical practice? Insights into Imaging. 2012;3(6):573-589. 
15. Ganeshan B, Panayiotou E, Burnand K, Dizdarevic S, Miles K. Tumour heterogeneity in non-small cell lung carcinoma assessed by CT texture analysis: a potential marker of survival. European Radiology. 2012;22(4):796-802.

16. Ganeshan B, Abaleke S, Young RCD, Chatwin CR, Miles KA. Texture analysis of non-small cell lung cancer on unenhanced computed tomography: initial evidence for a relationship with tumour glucose metabolism and stage. Cancer Imaging. 2010;10(1):137-143.

17. He X, Sahiner B, Gallas BD, Chen W, Petrick N. Computerized characterization of lung nodule subtlety using thoracic CT images. Physics in Medicine and Biology. 2014;59(4):897-910.

18. Skogen K, Ganeshan B, Good C, Critchley G, Miles K. Measurements of heterogeneity in gliomas on computed tomography relationship to tumour grade. Journal of Neuro-Oncology. 2013;111(2):213-219.

19. Ravanelli M, Farina D, Morassi M, Roca E, Cavalleri G, Tassi G, Maroldi R. Texture analysis of advanced non-small cell lung cancer (NSCLC) on contrast-enhanced computed tomography: prediction of the response to the first-line chemotherapy. European Radiology. 2013;23(12):3450-3455.

20. Aerts HJWL, Velazquez ER, Leijenaar RTH, Parmar C, Grossmann P, Cavalho S, Bussink J, Monshouwer R, Haibe-Kains B, Rietveld D, Hoebers F, Rietbergen MM, Leemans CR, Dekker A, Quackenbush J, et al. Decoding tumour phenotype by noninvasive imaging using a quantitative radiomics approach. Nature Communications. 2014;5.

21. Chae H-D, Park CM, Park SJ, Lee SM, Kim KG, Goo JM. Computerized Texture Analysis of Persistent PartSolid Ground-Glass Nodules: Differentiation of Preinvasive Lesions from Invasive Pulmonary Adenocarcinomas. Radiology. 2014:132187.

22. Fried DV, Tucker SL, Zhou S, Liao Z, Mawlawi O, Ibbott G, Court LE. Prognostic Value and Reproducibility of Pretreatment CT Texture Features in Stage III Non-Small Cell Lung Cancer. International Journal of Radiation Oncology*Biology*Physics. 2014.

23. Vaidya M, Creach KM, Frye J, Dehdashti F, Bradley JD, El Naqa I. Combined PET/CT image characteristics for radiotherapy tumor response in lung cancer. Radiotherapy and Oncology. 2012;102(2):239-245.

24. Aerts HJWL, Bussink J, Oyen WJG, van Elmpt W, Folgering AM, Emans D, Velders M, Lambin P, De Ruysscher D. Identification of residual metabolic-active areas within NSCLC tumours using a preradiotherapy FDG-PET-CT scan: A prospective validation. Lung Cancer. 2012;75(1):73-76.

25. van Elmpt W, Das M, Hüllner M, Sharifi H, Zegers CML, Reymen B, Lambin P, Wildberger JE, Troost EGC, Veit-Haibach $P$, De Ruysscher D. Characterization of tumor heterogeneity using dynamic contrast enhanced CT and FDG-PET in non-small cell lung cancer. Radiotherapy and Oncology. 2013;109(1):65-70.

26. Balagurunathan Y, Gu Y, Wang H, Kumar V, Grove O, Hawkins S, Kim J, Goldgof DB, Hall LO, Gatenby RA, Gillies RJ. Reproducibility and Prognosis of Quantitative Features Extracted from CT Images. Translational Oncology. 2014;7(1):72-87.

27. Ball DL, Fisher RJ, Burmeister BH, Poulsen MG, Graham PH, Penniment MG, Vinod SK, Krawitz HE, Joseph DJ, Wheeler GC, McClure BE. The complex relationship between lung tumor volume and survival in patients with non-small cell lung cancer treated by definitive radiotherapy: A prospective, observational prognostic factor study of the Trans-Tasman Radiation Oncology Group (TROG 99.05). Radiotherapy and Oncology. 2013;106(3):305-311.

28. Etiz D, Marks LB, Zhou S-M, Bentel GC, Clough R, Hernando ML, Lind PA. Influence of tumor volume on survival in patients irradiated for non-small-cell lung cancer. International Journal of Radiation Oncology* Biology* Physics. 2002;53(4):835-846.

29. Alexander BM, Othus M, Caglar HB, Allen AM. Tumor Volume Is a Prognostic Factor in Non-Small-Cell Lung Cancer Treated With Chemoradiotherapy. International Journal of Radiation Oncology • Biology • Physics. 2011;79(5):1381-1387.

30. Mirsadraee $\mathrm{S}$. The 7th lung cancer TNM classification and staging system: Review of the changes and implications. World Journal of Radiology. 2012;4(4):0.

31. Oken MM, Creech RH, Tormey DC, Horton J, Davis TE, McFadden ET, Carbone PP. Toxicity and response criteria of the Eastern Cooperative Oncology Group. American Journal of Clinical Oncology. 1982;5(6):649-655. 
32. Deasy JO, Blanco Al, Clark VH. CERR: a computational environment for radiotherapy research. Medical physics. 2003;30(5):979-985.

33. De Jay N, Papillon-Cavanagh S, Olsen C, El-Hachem N, Bontempi G, Haibe-Kains B. mRMRe: an R package for parallelized mRMR ensemble feature selection. Bioinformatics. 2013;29(18):2365-2368.

34. Schröder MS, Culhane AC, Quackenbush J, Haibe-Kains B. survcomp: an R/Bioconductor package for performance assessment and comparison of survival models. Bioinformatics. 2011;27(22):3206-3208.

35. Lumley T. rmeta.; 2012.

36. Gentleman RC, Carey VJ, Bates DM, Bolstad B, Dettling M, Dudoit S, Ellis B, Gautier L, Ge Y, Gentry J, Hornik K, Hothorn T, Huber W, lacus S, Irizarry R, et al. Bioconductor: open software development for computational biology and bioinformatics. Genome Biology. 2004;5(10):R80.

37. Harrell FE, Califf RM, Pryor DB, Lee KL, Rosati RA. Evaluating the yield of medical tests. JAMA: the journal of the American Medical Association. 1982;247(18):2543-2546.

38. Benjamini Y, Hochberg Y. Controlling the False Discovery Rate: A Practical and Powerful Approach to Multiple Testing. Journal of the Royal Statistical Society. Series B (Methodological). 1995;57(1):289-300.

39. Anon. $R$ Core Team (2013). R: A language and environment for statistical computing. $R$ Foundation for Statistical Computing, Vienna, Austria. ISBN 3-900051-07-0, URL http://www.R-project.org/. 



\section{Chapter}

\section{CT-based radiomic analysis of stereotactic body radiation therapy patients with lung cancer}

Published in: Radiotherapy and Oncology (2016)

CT-based radiomic analysis of stereotactic body radiation therapy patients with lung cancer Huynh Elzabeth*, Thibaud P. Coroller*, Narayan Vivek, Hou Ying, Romano John, Franco Idalid, Raymond H. Mak*, Hugo J.W.L. Aerts* 


\section{ABSTRACT}

Background: Radiomics uses a large number of quantitative imaging features that describe tumor phenotype to develop imaging biomarkers for clinical outcomes. Radiomic analysis of pre-treatment computed-tomography (CT) scans was investigated to identify imaging predictors of clinical outcomes in early stage non-small cell lung cancer (NSCLC) patients treated with stereotactic body radiation therapy (SBRT).

Materials and methods: CT images of 113 stage I-II NSCLC patients treated with SBRT were analysed. Twelve radiomic features were selected based on stability and variance. The association of features with clinical outcomes and their prognostic value (using the concordance index $(\mathrm{Cl})$ ) was evaluated. Radiomic features were compared with conventional imaging metrics (tumor volume or diameter) and clinical parameters.

Results: Overall survival was associated with two conventional features (volume and diameter) and two radiomic features (LoG 3D run low gray level short run emphasis and stats median). One radiomic (Wavelet LLH stats range) feature was significantly prognostic for distant metastasis $(\mathrm{Cl}=0.67$, q-value $<0.1)$, while none of the conventional and clinical parameters were. Three conventional and four radiomic features were prognostic for survival.

Conclusion: This exploratory analysis demonstrates that radiomic features have potential to be prognostic for some outcomes that conventional imaging metrics cannot predict in SBRT patients. 


\section{INTRODUCTION}

Heterogeneous disease within and among patients demands a need for an individualized approach to cancer treatment. Precision medicine aims to design treatment plans tailored to the specific disease profile of the patient to improve outcomes. However, a major challenge for individualized treatment is the inability to accurately predict how a patient's disease will behave and respond to particular therapies prior to treatment [1].

Treatment plans for cancer patients involve one or several treatment modalities involving surgery, chemotherapy and/or radiation therapy (RT). For early stage non-small cell lung cancer (NSCLC) patients, the primary treatment is surgery; however, due to underlying comorbidities, medically inoperable patients are treated with hypofractionated RT, known as stereotactic body radiation therapy (SBRT), as the standard of care $[2,3]$. Compared to conventional RT, SBRT administers higher radiation doses over a hypofractionated scheme (e.g. 2 Gy/fraction over 30 fractions for conventional RT vs. 12-18 Gy/fraction over 3-5 fractions for SBRT). SBRT has demonstrated excellent local control, overall survival (OS) and cancer-specific survival (CSS). These promising treatment outcomes have motivated investigations comparing the efficacy of SBRT to surgery, as a potential alternative treatment for surgical candidates [4]. However, despite the successes of SBRT, some patients still develop distant metastases (DM) (13-23\%) and local recurrence (4-14\%) [5-11]. While early stage patients with larger tumors (i.e. stage IBIIA) who undergo surgical resection may receive adjuvant chemotherapy [12], patients with medically inoperable disease often have co-morbidities that limit their ability to tolerate systemic therapy. Therefore, systemic therapy is not a feasible global strategy for all SBRT patients and there is a need for a non-invasive patient stratification approach to identify those who are at highest risk of recurrence after SBRT. Identification of these patients prior to treatment would allow augmentation of their therapeutic approach with addition of systemic therapy and/or radiation dose intensification to reduce disease relapse rates and increase OS [13].

A novel method to classify patients could be based on tumor phenotype derived from medical imaging. Radiomics offers a non-invasive approach to precision medicine by extracting a large number of advanced quantitative features from medical images to assess the tumor phenotype $[1,14,15]$. It then involves comprehensive analyses of these features with clinical outcomes as potential prognostic indicators using robust and reproducible methodology [16-18] (Figure 1). Radiomics generates a unique imaging atlas of the tumor that is a quantification of the tumor phenotype and could provide superior prognostic power over current clinical imaging metrics (e.g. tumor diameter as a predictor of response). Radiomic features have been associated with tumor characteristics, such as genotype and protein expression [19-21], and have been prognostic of clinical outcomes [22-26]. 
Computed tomography (CT)-based radiomics has immense potential for developing imaging biomarkers for NSCLC patients treated with SBRT since it is the most widely used imaging modality in RT for treatment planning, guidance and follow-up. While quantitative CT imaging has been well reported for lung cancer diagnosis and management [27], there are a limited number of studies on predicting outcomes in lung cancer patients undergoing SBRT [28-32]. Analysis of baseline Hounsfield Units (HU) and changes in $\mathrm{HU}$ or textural features after SBRT have been investigated as prognostic indicators for radiation-induced lung damage [28-30, 32] and recurrence [31]. These studies have been limited in their reproducibility and prognostic power prior to SBRT, which would be important for optimizing individualized treatment plans to improve prognosis and/or prevent recurrent disease.

The aim of the current study is to apply an exploratory CT-based radiomics analysis to investigate imaging biomarkers of clinical outcomes in SBRT patients from pretreatment images. This approach could have a large impact for precision medicine, as radiomic biomarkers are non-invasive and can be applied to imaging data that are already acquired in clinical settings.

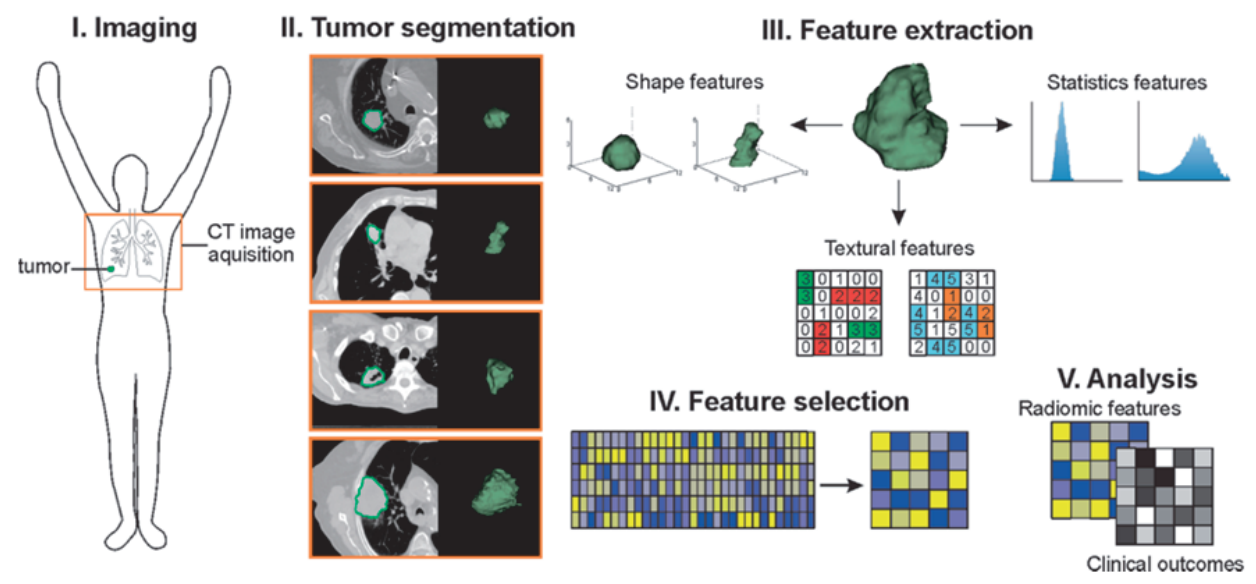

Figure 1. Schematic of the radiomics workflow. I) Computed tomography (CT) images of the lung are acquired of the patient. II) Primary tumors are segmented. III) Radiomics features are extracted from the whole tumor volume. IV) The high-dimensional feature set is reduced to a low-dimensional feature set solely based on stability and variance using a data-driven approach. V) The radiomic features and clinical outcomes are analysed to determine their prognostic value. 


\section{METHOD}

\section{Patient selection}

This study was Institutional Review Board (IRB) approved for analysis of non-small cell lung cancer (NSCLC) patients who underwent stereotactic body radiation therapy (SBRT) treatment at our institution between 2009 and 2014. This was a retrospective study and therefore, IRB approval was obtained for waiver of consent. The patient population was limited to patients with early stage NSCLC (overall stage I-II, NO). Patients that did not have a free breathing computed tomography $(C T)$ scan on file $(n=10)$, had greater than a 1-week duration between CT image acquisition and the start of treatment $(n=2)$, had multiple SBRT treatments and/or multiple tumor lesions $(n=17)$, or received induction chemotherapy $(n=2)$ were excluded from this study. In addition, patients who fulfilled any of the following criteria were also excluded: had metastases to the lung from other sites of primary disease $(n=30)$, locally recurrent disease $(n=5)$, had small cell lung cancer $(n=1)$ or atypical carcinoid $(n=1)$ histology, or were overall stage III or IV $(n=1)$. None of the patients received additional chemotherapy after SBRT. A total of 113 patients were included in the analysis and their characteristics can be found in Table 1.

SBRT treatment and clinical endpoints

All patients were treated with SBRT according to institutional standards. SBRT was restricted to peripheral tumors defined in Radiation Therapy and Oncology Group (RTOG) 0236 [9] and abdominal compression was used if tumor motion was greater than $1 \mathrm{~cm}$. Treatment planning was performed on 4D CT where the internal target volume was defined, and a planning target volume (PTV) with a $5 \mathrm{~mm}$ margin with no clinical target volume margin was created. For tumors close to the chest wall, patients received a dose of 10 to $12 \mathrm{~Gy} \times 5$ fractions, and 12 to $14 \mathrm{~Gy} \times 4$ fractions or $18 \mathrm{~Gy} \times 3$ fractions for all other tumors. One patient was unable to complete the full course of treatment due to death and only received 1 fraction of $18 \mathrm{~Gy}$ (delivered biologically effective dose of 50.4 Gy). Exac Trac, cone-beam CT and portal imaging using a linear accelerator were used for daily setup and image-guided treatment.

Follow-up chest CT scans with contrast (unless the patient had a contraindication to contrast, e.g. renal dysfunction or allergy) were performed every three to six months after treatment based on United States national guidelines [12] to assess tumor progression. Distant metastasis (DM) and locoregional recurrence (LRR) were evaluated. The spread of disease to sites outside of the lungs (e.g. brain) was considered DM. LRR was defined as any local, lobar, and/or regional (nodal) recurrence. Recurrence within the PTV was classified as local recurrence, whereas recurrence occurring in the same lobe as the primary tumor but outside of the SBRT treatment field was considered lobar recurrence, and regional recurrence was defined as hilar, mediastinal and supraclavicular lymph node recurrence (Figure 2). 

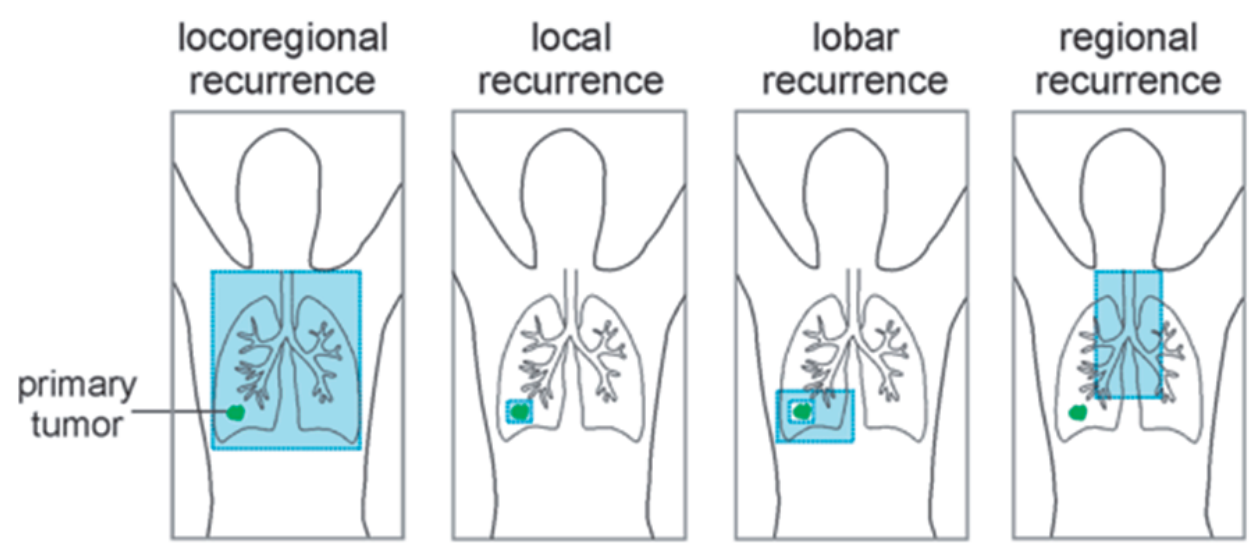

Figure 2. Schematic of sub-sites of recurrence. Locoregional recurrence considers all recurrence in the lungs, local recurrence refers to recurrence within the SBRT field, lobar recurrence refers to recurrence occurring in the same lobe as the primary but outside the SBRT field, and regional recurrence refers to recurrence in the lymph nodes in the lungs. The blue box highlights the region of interest for the indicated pattern of recurrence.

\section{CT image acquisition and tumor segmentation}

Free breathing CT images were acquired on a GE LightSpeed RT16 CT scanner (GE Medical Systems, Milwaukee, WI, USA) according to standard clinical scanning protocols. The most common imaging slice thickness and pixel spacing was $2.5 \mathrm{~mm}$ and $1.27 \mathrm{~mm}$ by $1.27 \mathrm{~mm}$, respectively. The primary tumor site was contoured on Eclipse software (Varian Medical Systems, Palo Alto, CA, USA). Tumors were manually contoured by E.H., V.A., and Y.H, and then individually verified by an expert radiation oncologist (R.H.M.).

\section{Radiomic feature extraction}

A set of 1605 radiomic features describing the tumor phenotype was extracted from the tumors in the free breathing CT images using an in-house Matlab 2013 (The Mathworks Inc., Natick, MA, USA) toolbox and 3D Slicer 4.4 .0 software [33]. A bin width of 25 Hounsfield units (HU) for textural features was used to increase sensitivity relative to the raw image. This discretization step reduces image noise and normalizes intensities across all patients, which allowed for a direct comparison of all calculated textural features. All CT voxels were resampled to $1 \times 1 \times 1 \mathrm{~mm}^{3}$ using a bicubic interpolation function prior to feature extraction to standardize the voxel spacing across the cohort. These features were organized into three categories including shape, statistics and textural features. A description of the radiomics features can be found in the supplementary material from previous studies $[22,23]$. Our feature set contained more features than Coroller et al. [22] as a result of adding textural features that had been calculated using Laplacian of Gaussian (LoG) filters. 
In order to compare the prognostic value of the radiomics features, commonly used clinical CT metrics ("conventional features") for tumor characteristics were also included in the study. These conventional features included tumor volume, maximum axial diameter (taken as the maximum diameter measured in a single 2D axial imaging slice) and maximum 3D diameter. The maximum axial diameter and maximum 3D diameter measurements were computed based on the 3D segmentations. The maximum 3D diameter was measured as the largest pairwise Euclidean distance, between voxels on the surface of the tumor volume. The maximum axial diameter is taken as the maximum 2D tumor diameter measured on an axial slice. Therefore, the diameters were measured such that the measured segment lay entirely within the solid tumor. The conventional features were not included in the feature dimension reduction process for the radiomic features. The prognostic value of the radiomic features to predict clinical outcomes were also compared against the prognostic value of clinical parameters, including age, gender, performance status and overall stage.

\section{Radiomic feature dimension reduction}

A two-step feature dimension reduction method was used for the radiomic features. First, stable features were selected using the test-retest Reference Image Database to Evaluate Therapy Response (RIDER) dataset [34]. The RIDER dataset consists of a series of CT images from 31 NSCLC patients obtained approximately 15 min apart in a similar position. This dataset was only used to select features that were stable through the test-retest assessment with an intraclass correlation coefficient (ICC) greater than 0.8 (using the "irr" package [35]), reducing the number of features to 855 stable features.

Second, Principal Component Analysis (PCA) and factor analysis was applied on our dataset using the "FactoMineR" package [36] to further reduce the resulting highdimensional dataset to a low-dimensional dataset while retaining most of the variation contained within the data and reducing redundancy. PCA creates a new principal component space using scores that describe the single value decomposition of the features. Scores that retained $95 \%$ of the variability from the stable features were selected. Furthermore, the features that correlated by at least $99 \%$ to the PCA scores were then selected. This feature dimension reduction process resulted in 12 radiomics features.

\section{Data analysis}

All statistical analyses were performed in R software version 3.2.2 [37]. Univariate analysis was conducted using the "survcomp" package [38, 39] version 1.16 from Bioconductor [40]. We analyzed the association between features and each outcome by comparing the feature distribution at the median time of event (24 months for OS and 12 months for LRR and DM). Patients that were censored or did not have an event before 
the considered time point were not considered in the assessment of the differences in the feature distribution. The number of patients excluded from the association analysis was 14 patients for DM, 25 patients for LRR, 26 patients for OS and 9 patients for CSS. A two-sided Wilcoxon rank-sum test was used to assess the difference in the features between patients with or without an event. Multiple testing correction was applied by the false discovery rate (FDR) procedure introduced by Benjamini and Hochberg [41, 42], where a q-value less than 0.1 was considered statistically significant. Boxplots are reported in Supplementary Figures 1-4. Estimates of survival and event-free probabilities were determined by the Kaplan-Meier method at the median time to event using the "survival" package [43].

The prognostic value of the imaging features was evaluated by calculating the concordance index $(\mathrm{Cl})$ using the "survcomp" package in $\mathrm{R}[38,39]$. The $\mathrm{Cl}$ was calculated from the feature values or clinical parameters and clinical outcome, incorporating the full time data for each outcome while accounting for censoring of the data [44, 45]. The $\mathrm{Cl}$ is a generalization of the area under the receiver operating characteristic curve (AUC) (with the incorporation of time) and a measure of the probability that between two randomly drawn samples, the sample with the higher value (e.g. of an imaging feature) will have a higher likelihood of the event (e.g. DM). A Cl greater than 0.5 indicates direct proportionality between the feature value and clinical outcome. Furthermore, a $\mathrm{Cl}$ less than 0.5 indicates inverse proportionality (the lower the value, the higher the likelihood of the event). The Noether's test was used to compute the $p$-value to determine the significance of the $\mathrm{Cl}$ from random $(\mathrm{Cl}=0.5)$. Multiple testing correction was applied by the false discovery rate (FDR) procedure introduced by Benjamini and Hochberg [41, 42], where a q-value less than 0.1 was considered statistically significant.

Multivariate models were generated using stratified cross validation by evaluating 100 iterations of each type of model (clinical, conventional, radiomic, etc.) using a partition in the dataset of $80 \%$ training and $20 \%$ validation. We used a conservative partition in the dataset such that the training and validation datasets contained roughly the same proportion of events. The reduced feature set determined by PCA was applied in each training dataset and a set of 3 features were selected from the respective training dataset only to avoid bias, based on the highest univariate performances to create each model, and evaluated on the validation dataset. The reduced feature set determined by PCA was applied in each training dataset and a set of 3 features were selected only from the respective training dataset to avoid bias, based on the highest univariate performances to create each model. The radiomics model generated from the training dataset was evaluated on the validation dataset. Three features were selected for the radiomics signature because choosing 3 features would generate a similar signature for each iteration and capture the best performing features, without the addition of excessive noise. Furthermore, based on the univariate performance of the overall cohort, the top 3 
performing features had $\mathrm{Cl}$ greater than 0.6.The $\mathrm{p}$-values for these models were determined using a permutation test with 1000 iterations.

\section{RESULTS}

A total of 113 early stage non-small cell lung cancer (NSCLC) patients treated with stereotactic body radiation therapy (SBRT) were included in the analysis with a median age of 74 (range: 47-89) at the initiation of treatment. The patient population was nearly equally divided by gender with $50.4 \%$ female and $49.6 \%$ male. All patients received SBRT with a median biologically effective dose of 151.2 Gy (range: 50.4-151.2 Gy). None of the patients received chemotherapy. The median follow-up time was 20.8 months (range: 0.0-47.8 months) and the median follow-up time of survivors was 25.2 months (range: $3.3-47.8$ months). $20.4 \%$ of patients developed distant metastasis (DM) and $21.2 \%$ developed locoregional recurrence (LRR). The median time to DM and LRR was 10.0 months (range: $2.0-37.7$ months) and 8.8 months (2.0-26.4 months), respectively. Overall survival (OS) was $52.2 \%$ with a median survival time of 22.5 months (range: 0.03-47.8). The 2-year estimates for DM, LRR and OS were $74.0 \%, 70.9 \%$ and $61.8 \%$, respectively. Patient characteristics and clinical outcomes are shown in Table 1.

Table 1.Patient, tumor, and treatment characteristics and clinical outcomes

\begin{tabular}{|c|c|c|}
\hline & & $\begin{array}{l}\text { Total ( } n=113 \text { patients) } \\
\text { median (range) or number }(\%)\end{array}$ \\
\hline \multirow{12}{*}{$\begin{array}{l}\text { Patient } \\
\text { characteristics }\end{array}$} & Age & $74(47-89)$ \\
\hline & Gender & \\
\hline & Female/ Male & $57 / 56$ (50.4/ 49.6) \\
\hline & Ethnicity & \\
\hline & African-American & $8(7.1)$ \\
\hline & Asian & $2(1.8)$ \\
\hline & Caucasian & $103(91.1)$ \\
\hline & Smoking & \\
\hline & Never/ Current/ Former & $3 / 27 / 83$ (2.7/ 23.9/ 73.4) \\
\hline & Pack-years & $50(0.4-180.0)$ \\
\hline & Performance status & \\
\hline & $0 / 1 / 2 / 3$ & $17 / 51 / 39 / 6$ (15.0/ 45.1/ 34.5/5.3) \\
\hline \multirow{10}{*}{$\begin{array}{l}\text { Tumor } \\
\text { characteristics }\end{array}$} & Overall stage & \\
\hline & $|A /| B /|| A$ & 95/ 17/ 1 (84.1/ 15.0/ 0.9) \\
\hline & T stage & \\
\hline & $\mathrm{T} 1 \mathrm{a} / \mathrm{T} 1 \mathrm{~b} / \mathrm{T} 2 \mathrm{a} / \mathrm{T} 2 \mathrm{~b}$ & $67 / 27 / 18 / 1$ (59.3/ 23.9/ 15.9/ 0.9) \\
\hline & Histology & \\
\hline & Adenocarcinoma & $48(42.5)$ \\
\hline & Adenosquamous carcinoma & $1(0.9)$ \\
\hline & Squamous cell carcinoma & $27(23.9)$ \\
\hline & Undifferentiated NSCLC & $17(15.0)$ \\
\hline & No pathology & $20(17.7)$ \\
\hline
\end{tabular}




\begin{tabular}{|c|c|c|}
\hline & & $\begin{array}{l}\text { Total ( } \mathrm{n}=113 \text { patients) } \\
\text { median (range) or number (\%) }\end{array}$ \\
\hline \multirow[t]{5}{*}{$\begin{array}{l}\text { Treatment } \\
\text { characteristics }\end{array}$} & $\begin{array}{l}\text { SBRT technique } \\
\text { 3D Conformal / VMAT }\end{array}$ & $85 / 28(75.2 / 24.8)$ \\
\hline & Prescribed radiation dose (Gy) & $54(18-60)$ \\
\hline & Radiation dose per fraction (Gy) & $18(10-18)$ \\
\hline & $\begin{array}{l}\text { Number of radiation fractions } \\
1 / 3 / 4 / 5\end{array}$ & $1 / 67 / 2 / 43(0.9 / 59.3 / 1.8 / 38.0)$ \\
\hline & Delivered biologically effective dose (Gy) & $151.2(50.4-151.2)$ \\
\hline \multirow[t]{7}{*}{ Clinical outcomes } & Follow-up time (months) & $20.8(0.0-47.8)$ \\
\hline & Follow-up time of survivors (months) & $25.2(3.3-47.8)$ \\
\hline & $\begin{array}{l}\text { Distant metastasis (DM) } \\
\text { No/ Yes } \\
\text { Time to event (months) } \\
\text { Estimate of freedom from DM at } 2 \text { years }\end{array}$ & $\begin{array}{l}90 / 23(79.6 / 20.4) \\
10.0(2.0-37.7) \\
74.0 \%\end{array}$ \\
\hline & $\begin{array}{l}\text { Locoregional recurrence (LRR) } \\
\text { No/ Yes } \\
\text { Time to event (months) } \\
\text { Estimate of freedom from LRR at } 2 \text { years }\end{array}$ & $\begin{array}{l}89 / 24(78.8 / 21.2) \\
8.8(2.0-26.4) \\
70.9 \%\end{array}$ \\
\hline & $\begin{array}{l}\text { Site of recurrence } \\
\text { Regional/ Lobar/ Local }\end{array}$ & $17 / 13 / 9$ \\
\hline & $\begin{array}{l}\text { Survival } \\
\text { No/ Yes } \\
\text { Time to event (months) } \\
\text { Estimate of survival at } 2 \text { years }\end{array}$ & $\begin{array}{l}54 / 59(47.8 / 52.2) \\
22.5(0.03-47.8) \\
61.8 \%\end{array}$ \\
\hline & $\begin{array}{l}\text { Cause of death } \\
\text { Cancer/ Other causes/ Unknown cause }\end{array}$ & $16 / 20 / 18$ \\
\hline
\end{tabular}

The full radiomic feature set was reduced to twelve radiomic features that were selected based on feature stability, preserving the variance in the data and minimizing redundancy among the features. A total of 15 imaging features ( 3 conventional features and 12 radiomic features) and 4 clinical parameters (age, gender, performance status, overall stage) were included in our analysis. The association between imaging features and clinical parameters with the main clinical outcomes (DM, LRR, OS, cancer-specific survival (CSS)) were evaluated at the median time of the event (Figure 3). None of the imaging features or clinical parameters were significantly associated with DM, LRR or CSS. Four imaging features were associated with OS including two conventional features (describing tumor diameter and volume), one textural feature (describing characteristics of tumor heterogeneity) and one statistics features (describing image intensity median). One clinical parameter, performance status, was significantly associated with OS. The features and their corresponding q-values can be found in Supplementary Table 1. 


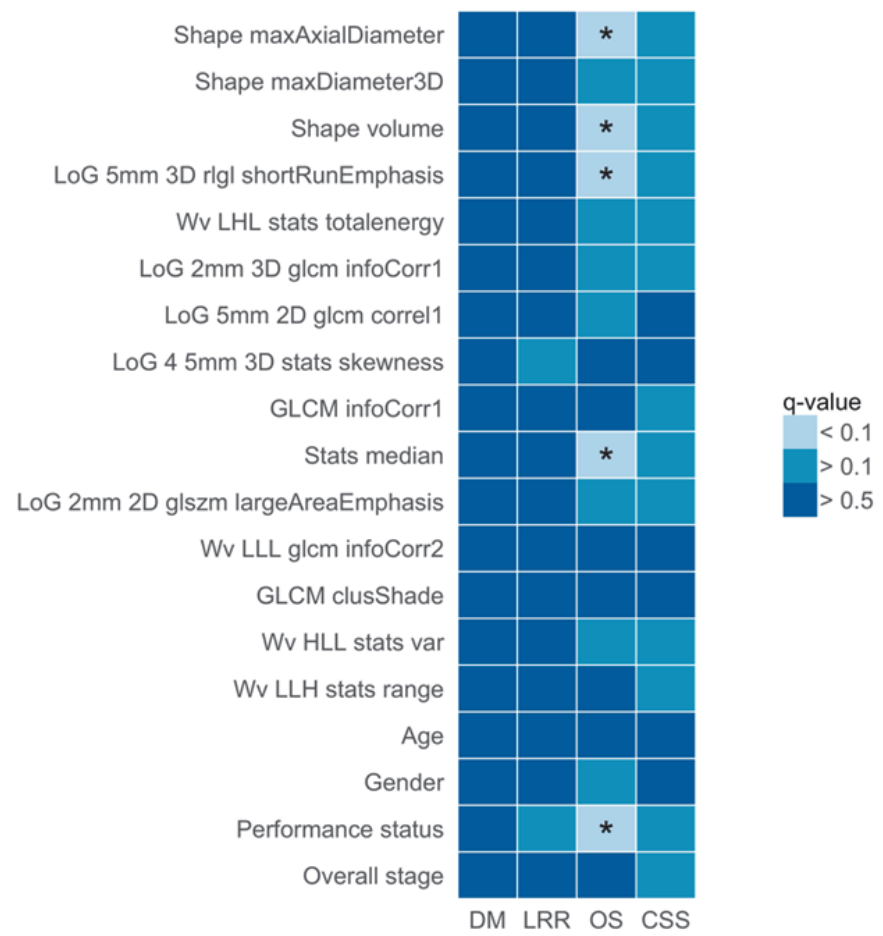

Figure 3. Heatmap of the association between imaging features and clinical parameters, and clinical outcomes. Imaging features (3 conventional features and 12 radiomic features) were evaluated for distant metastasis (DM), locoregional recurrence (LRR), overall survival (OS) and cancer-specific survival (CSS) with the corresponding q-value indicated (Wilcoxon rank-sum test, FDR corrected). The considered time point for DM, LRR and OS was the median time of event (12 months, 12 months, 24 months and 24 months, respectively). $* \mathrm{q}<0.1$.

The prognostic power of the imaging features was determined using the concordance index $(\mathrm{Cl})$ for each clinical outcome and evaluating its significance from random. Conventional and radiomic features for DM and LRR are shown in Figure 4. A statisticsbased radiomic feature was prognostic for DM and none of the features were significant for LRR. Notably, none of the conventional features had significant prognostic value (Supplementary Table 2). The prognostic radiomic feature for DM described the range of voxel intensities (Wavelet LLH stats range, $\mathrm{Cl}=0.67$, q-value $=0.067$ ). Sub-sites of LRR were also evaluated, in which none of the conventional features were prognostic. However, five radiomic features were prognostic of local recurrence (two statistics features and three textural features) and three radiomic features (one statistics feature and two textural features) for lobar recurrence. None of the imaging features (conventional or radiomic) were prognostic for regional recurrence (Supplementary Figure 5 and Supplementary Table 3). 


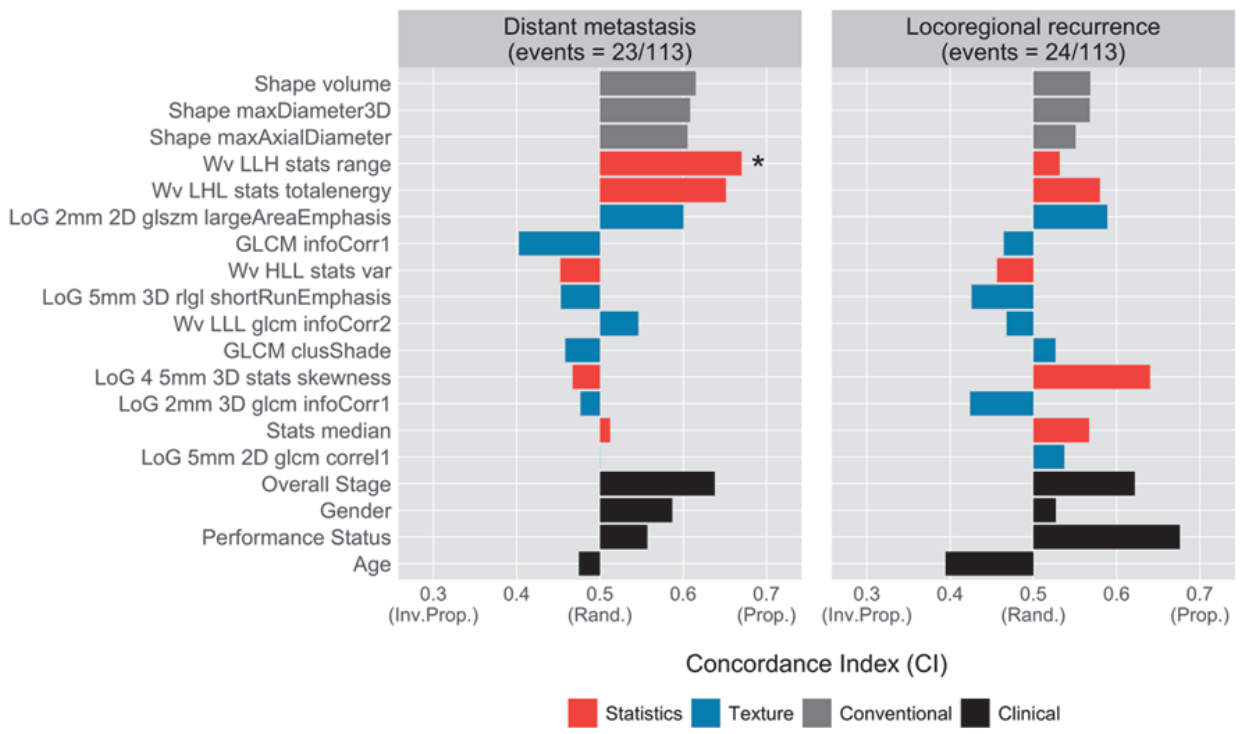

Figure 4. Prognostic values of imaging features for distant metastasis (DM) and locoregional recurrence (LRR) in SBRT NSCLC patients. Radiomic features are shown in red (statistics features) and blue (textural features), clinical parameters are shown in black and conventional features are shown in grey. The concordance index $(\mathrm{Cl})$ is plotted for each feature. "Prop." indicates direct proportionality and "Inv. Prop." denotes inverse proportionality. *q-value $<0.1$ (Noether test).

For OS, the Cls of 7 imaging features (3 conventional, 4 radiomic) were significant from random (Figure 5). The majority of features had Cls less than 0.5 , which indicated that the feature values were inversely proportional to the probability of survival. The $\mathrm{Cls}$ of the conventional features ranged from 0.35-0.37 (q-value $=0.0024-0.0036$ ). The best performing radiomic feature for OS (stats median) had a $\mathrm{Cl}$ of 0.33 (q-value $=0.0016$ ). When the patient population was reduced to evaluate CSS, the number of prognostic features was reduced to 4 ( 1 conventional, 3 radiomic) (Figure 5). The four prognostic imaging features for CSS were common with OS. The values of these features can be found in Supplementary Table 4. The prognostic values of the clinical parameters were also assessed. None of the clinical parameters were prognostic for DM or LRR with $\mathrm{Cl}$ ranging from 0.48-0.64 (q-value $=0.72-0.81$ ) and 0.39-0.68 (q-value $=0.29-0.80$ ), respectively (Supplementary Table 5 ). Performance status was significantly prognostic for local recurrence $(\mathrm{Cl}=0.82$, q-value $=0.00033)$ (Supplementary Table 6). Age $(\mathrm{Cl}=$ 0.35 , q-value $=0.076)$ and performance status $(\mathrm{Cl}=0.76$, q-value $=0.0090)$ were significantly prognostic for lobar recurrence. None of the clinical parameters were prognostic for regional recurrence with $\mathrm{Cls}$ ranging from 0.40-0.62 (q-value $=0.80$ ). $\mathrm{Age}(\mathrm{Cl}=0.34$, $q$-value $=0.0011)$ and performance status $(\mathrm{Cl}=0.33$, q-value $=0.0052)$ were significantly prognostic for OS and age was prognostic for $\operatorname{CSS}(\mathrm{Cl}=0.37$, q-value $=0.028)$ (Supplementary Table 6). 


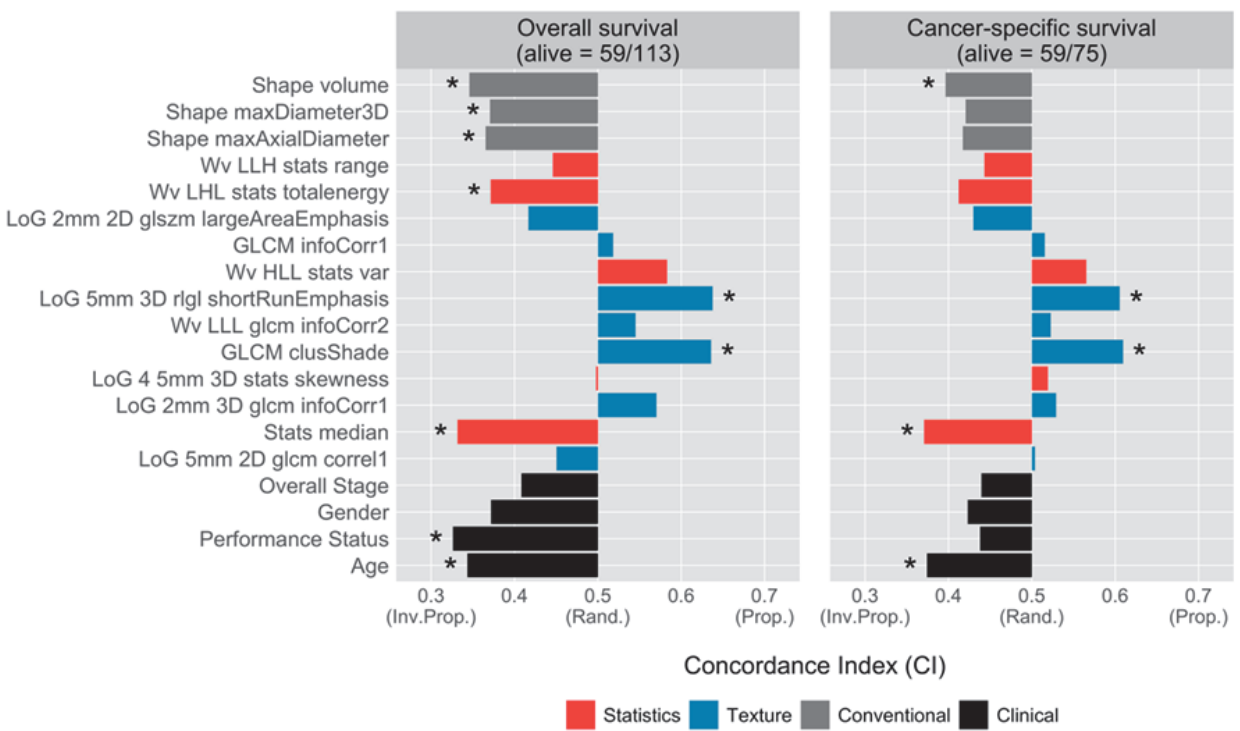

Figure 5. Prognostic values of imaging features for survival in SBRT NSCLC patients. The clinical outcomes evaluated were overall survival (left) and cancer-specific survival (right). Radiomic features are shown in red (statistics features) and blue (textural features), clinical parameters are shown in black and conventional features are shown in grey. ${ }^{*}$ q-value $<0.1$ (Noether test).

Multivariate models were generated for DM based on clinical parameters, conventional imaging features and radiomic features using cross validation with 100 iterations. Radiomic multivariate models were generated by applying the reduced radiomic feature set to the training cohort alone, to avoid bias, and evaluating their univariate performances. A radiomic signature composed of the top three univariately performing features from the training dataset was then evaluated on the validation cohort for each iteration. We evaluated DM as the clinical outcome since univariate analysis identified a prognostic radiomic feature for this outcome and it had the most clinical relevance since $13-23 \%$ of patients will develop DM [5-11]. The prognostic performance of the radiomics model (median $\mathrm{Cl}$ of 0.67 ) was higher than the conventional (median $\mathrm{Cl}$ of 0.62 , p-value $=0.11$ ) and clinical (median $\mathrm{Cl}$ of 0.62 , p-value $=0.18$ ) models, although it was not statistically significant (Supplementary Figure 6). Within the 100 iterations of cross validation, the most frequently occurring features in the radiomics model was Wv LLH stats range, followed by Wv LHL stats totalenergy (Supplementary Figure 7). Wv LLH was included in 94 of the 100 models and Wv LHL stats totalenergy was included in 88 of the 100 models (Supplementary Table 8 ). These features were also the highest univariately performing features for the overall cohort (Figure 3). 


\section{DISCUSSION}

Understanding the behaviour of a tumor in response to a particular therapy is crucial for precision medicine in order to design an optimized treatment plan. Medical imaging is not only used for diagnosis and monitoring of cancer patients, but also image-based tumor size metrics derived from these images are used as prognostic indicators of treatment response. However, these current clinical metrics do not describe and exploit all of the tumor information captured in these images. Radiomics assesses a large number of imaging features that characterize the tumor phenotype, using descriptors beyond tumor size, to predict clinical outcomes with increased prognostic power. This approach could be important for patients treated with radiation therapy where treatment optimization is not only based on physical parameters but also the use of biological information, such as those derived from medical images regarding the tumor phenotype, to stratify patients who are at risk of disease recurrence [46]. In particular, this is imperative for early non-small cell lung cancer (NSCLC) patients treated with stereotactic body radiation therapy (SBRT) in which $13-23 \%$ of patients develop distant metastasis (DM) and $4-14 \%$ of patients experience local recurrence after treatment [5-11]. Randomized trials have demonstrated a modest benefit of adjuvant chemotherapy for higher risk, early stage NSCLC patients after surgery [47], however, patients treated with SBRT are medically inoperable and usually have limited tolerance to systemic therapy. Therefore, only the patients with the highest risk of recurrence after SBRT should have additional or intensified treatment to prevent recurrence. Radiomics could potentially identify these high-risk patients.

The main objective of the current study was to explore the feasibility of applying radiomics for identifying disease recurrence in NSCLC patients who underwent SBRT and to compare their prognostic power to conventional imaging metrics, such as tumor diameter. To our knowledge, this is the first computed tomography (CT)-based radiomics study on SBRT NSCLC patients investigating the prognostic value of features obtained prior to treatment for clinical outcomes. Since none of the SBRT patients in our study received chemotherapy, the future potential of this study could lead to the identification of high risk patients for recurrence prior to SBRT, where the addition of chemotherapy or an intensified radiation dose to the treatment plan may reduce the risk of these adverse outcomes [13]. While none of the imaging features or clinical parameters were associated with DM or LRR, we found that a statistics-based radiomic feature (range) was prognostic of DM. Furthermore, none of the conventional features or clinical parameters were prognostic of DM, thereby highlighting the potential value of radiomics in outcome prediction of SBRT patients. This result also highlights the notion that a feature may not be significantly associated with a particular outcome but may have strong prognostic power for that same outcome. The characteristics of the distribution that qualify a feature as significantly associated with an outcome are different from the 
properties of the distribution that qualify a feature as significantly more predictive than a random guess [48]. The statistics-based feature that was prognostic for DM was Wavelet LLH stats range, which measures the range of voxel intensity values, and in the case of DM, a larger range of intensity values suggests a higher probability of developing DM. A tumor with a high range of voxel intensities may appear more heterogeneous than one that has a low range of voxel intensities and thus, also has a higher probability of developing DM. This suggests that the phenotypic information captured in radiomic features may be prognostic for clinical outcomes that cannot currently be predicted using clinical imaging metrics, in the context of our current study. Therefore, this statistics feature could potentially serve as important imaging biomarker for DM in NSCLC patients treated with SBRT.

In addition to LRR, we also performed a subset analysis on different sub-sites of recurrence, including regional, lobar and local recurrences. These patterns of recurrence are of interest due to their distinctive underlying biological causes that may have different preventative treatments if identified prior to SBRT. For example, a high risk of local recurrence would suggest high radiation resistance of the tumor, whereas a high risk for regional recurrence would be indicative of the propensity of the tumor to spread to lymph nodes. We were able to identify radiomic features that were prognostic indicators of local and lobar recurrence, whereas none of the conventional features were able to perform significantly different than random. However, due to the relatively low number of LRR events, these findings would require further investigation and validation in a larger patient cohort.

Evaluation of conventional, clinical and radiomic multivariate models for DM demonstrated that radiomics models could potentially outperform other models, since in our dataset, the radiomics model had a median $\mathrm{Cl}$ greater than the other models, although the difference was not statistically significant. The features that occurred most frequently in the radiomics multivariate models (WV LLH stats range and WV LHL stats totalenergy) may be of future interest for a potential imaging biomarker for DM. Overall, this demonstrates that radiomic models have potential for predicting DM, however, this needs to be confirmed in larger cohorts. Nonetheless, our current work is an exploratory analysis to demonstrate that radiomics can be applied for NSCLC patients treated with SBRT and that it has potential for identification of patients with a high risk of recurrence.

CT-based quantitative imaging applied for lung cancer SBRT patients has focussed primarily on patient radiosensitivity and radiation toxicity [28-32]. Recently, a study by Mattonen et al. used CT texture analysis to identify a predictor of tumor recurrence in 22 NSCLC patients treated with SBRT [31]. They reported that CT textural features extracted from post-SBRT images acquired 2-5 months after treatment could differentiate tumor recurrence from radiation induced lung injury with an area under the receiver 
operating characteristic curve (AUC) ranging from 0.79-0.81. It is important to note that this approach is distinctly different from our current study. Their purpose for differentiating tumor recurrence from lung injury after SBRT was for earlier identification of patients requiring salvage therapies, whereas, our objective is to identify patients with a high risk of recurrence before they begin SBRT treatment. Our approach would allow optimization of the patient's treatment plan prior to the initiation of treatment to prevent recurrence.

Lastly, overall survival (OS) is an important clinical outcome that usually provides an indication of therapeutic efficacy. We investigated the association and prognostic value of the imaging features for survival. Although OS did have an association with conventional features, OS was also associated with numerous radiomic textural and statistics features. The highest ranked radiomic features had similar prognostic values with the conventional features. The contribution of therapeutic efficacy to OS may be difficult to assess in this patient population due to a median age of 74 , which is reflected in the cause of death. Only $29.6 \%$ of deaths were due to the cancer, whereas other patient deaths were due to other causes, such as co-morbidities, or unknown causes (Table 1). Therefore, we reduced the patient cohort to cancer-related deaths and observed a decrease in the number of prognostic imaging features for cancer-specific survival (CSS), however, the radiomic features still performed similarly to the conventional features. This suggests that radiomic features could potentially provide additive information to the current clinical imaging metrics.

A limitation of many quantitative imaging studies on SBRT patients, including our study, is the patient cohort size. Over the past decade, there has been an increasing use of SBRT, especially for lung cancer patients. Pan et al. reported that nearly half of the physicians surveyed on using SBRT in the United States adopted the technique in 2008 or later [49]. The clinical implementation of a new technique or treatment often begins with limited enrolment of patients and a steady increase in treated patients with each successive year. The dataset in our current study is from patients treated with SBRT between 2009 and 2014, resulting in a cohort size of 113 patients. Therefore, due to the number of patients in our dataset, this current study is exploratory and requires further validation in a larger patient cohort in the future.

The patient cohort size also limited our feature reduction method to an unsupervised method that was not based on the patient outcomes. We chose to use principal component analysis (PCA) in order to maintain the meaning of the features (through capturing the variance in the data). The choice of PCA is arguable as it will likely not give the best features for the analysis but it gives a reduced subset of features using an unbiased selection toward the outcomes. These features can then be evaluated for their prognostic performance, as we have demonstrated univariately. The suitability of PCA for di- 
mensionality reduction of the data in this paper is unknown since the data may lie on a nonlinear manifold.

Another shortcoming of our study is that it was limited to a single institution and tumors were delineated and verified by single individuals. Image acquisition and evaluation of clinical outcomes may differ between different institutions. Therefore, future work will be required to evaluate these radiomic features in pooled analyses of independent SBRT datasets to evaluate the generalizability of this study to all early stage NSCLC patients. In addition, the sensitivity of the prognostic accuracy of the features in this study, with respect to tumor contouring variability, is unknown and needs to be measured in a future study involving multiple observers. Lastly, since we evaluated early stage NSCLC (stage I-II) treated with SBRT, this was a limitation for extracting radiomic features due to the small size of the tumors (median 3D diameter $=2.7 \mathrm{~cm}$, range: 0.85 $7.1 \mathrm{~cm}$ ) and hence, may impact the prognostic performance of the radiomic features. However, despite these limitations, we were still able to identify radiomic features that were prognostic over a random guess despite this limitation.

This study investigated the potential of radiomic features as prognostic indicators of clinical outcomes for early stage NSCLC patients treated with SBRT, and compared their performance with clinical metrics derived from medical images (conventional features) and clinical parameters. On our data set, radiomic features were prognostic indicators for distant metastases, whereas conventional and clinical features were not, and had similar prognostic power to conventional features for survival. This demonstrates that radiomics may have importance in precision medicine for early stage NSCLC SBRT patients by developing prognostic imaging biomarkers for clinical outcomes, although these findings require further exploration and validation in larger cohorts and independent validation datasets. Identification of patients with the highest risk of recurrent disease prior to SBRT treatment would allow clinicians to personalize their treatment plan to reduce the risks of these outcomes and improve survival.

\section{ACKNOWLEDGEMENTS}

Authors acknowledge financial support from the National Institute of Health (NIH-USA U24CA194354, and NIH-USA U01CA190234). This project was partially funded by the Kaye Scholar Award and the Brigham and Women's Hospital Department of Radiation Oncology Clinical Translational Grant. The study sponsors had no role in study design, data collection and analysis, decision to publish, or preparation of the manuscript. 


\section{REFERENCES}

[1] Lambin P, van Stiphout RG, Starmans MH, Rios-Velazquez E, Nalbantov G, Aerts HJ, et al. Predicting outcomes in radiation oncology--multifactorial decision support systems. Nature reviews Clinical oncology. 2013;10:27-40.

[2] Crino L, Weder W, van Meerbeeck J, Felip E, Group EGW. Early stage and locally advanced (nonmetastatic) non-small-cell lung cancer: ESMO Clinical Practice Guidelines for diagnosis, treatment and follow-up. Annals of oncology : official journal of the European Society for Medical Oncology / ESMO. 2010;21 Suppl 5:v103-15.

[3] Potters L, Kavanagh B, Galvin JM, Hevezi JM, Janjan NA, Larson DA, et al. American Society for Therapeutic Radiology and Oncology (ASTRO) and American College of Radiology (ACR) practice guideline for the performance of stereotactic body radiation therapy. International journal of radiation oncology, biology, physics. 2010;76:326-32.

[4] Solda F, Lodge M, Ashley S, Whitington A, Goldstraw P, Brada M. Stereotactic radiotherapy (SABR) for the treatment of primary non-small cell lung cancer; systematic review and comparison with a surgical cohort. Radiotherapy and oncology : journal of the European Society for Therapeutic Radiology and Oncology. 2013;109:1-7.

[5] Baumann P, Nyman J, Hoyer M, Wennberg B, Gagliardi G, Lax I, et al. Outcome in a prospective phase II trial of medically inoperable stage I non-small-cell lung cancer patients treated with stereotactic body radiotherapy. Journal of clinical oncology : official journal of the American Society of Clinical Oncology. 2009;27:3290-6.

[6] Chi A, Liao Z, Nguyen NP, Xu J, Stea B, Komaki R. Systemic review of the patterns of failure following stereotactic body radiation therapy in early-stage non-small-cell lung cancer: clinical implications. Radiotherapy and oncology : journal of the European Society for Therapeutic Radiology and Oncology. 2010;94:1-11.

[7] Mak RH, Hermann G, Lewis JH, Aerts HJ, Baldini EH, Chen AB, et al. Outcomes by tumor histology and KRAS mutation status after lung stereotactic body radiation therapy for early-stage non-small-cell lung cancer. Clinical lung cancer. 2015;16:24-32.

[8] Fakiris AJ, McGarry RC, Yiannoutsos CT, Papiez L, Williams M, Henderson MA, et al. Stereotactic body radiation therapy for early-stage non-small-cell lung carcinoma: four-year results of a prospective phase Il study. International journal of radiation oncology, biology, physics. 2009;75:677-82.

[9] Timmerman R, Paulus R, Galvin J, Michalski J, Straube W, Bradley J, et al. Stereotactic body radiation therapy for inoperable early stage lung cancer. Jama. 2010;303:1070-6.

[10] Onishi H, Araki T, Shirato H, Nagata Y, Hiraoka M, Gomi K, et al. Stereotactic hypofractionated high-dose irradiation for stage I nonsmall cell lung carcinoma: clinical outcomes in 245 subjects in a Japanese multiinstitutional study. Cancer. 2004;101:1623-31.

[11] Grills IS, Mangona VS, Welsh R, Chmielewski G, Mclnerney E, Martin S, et al. Outcomes after stereotactic lung radiotherapy or wedge resection for stage I non-small-cell lung cancer. Journal of clinical oncology : official journal of the American Society of Clinical Oncology. 2010;28:928-35.

[12] Network NCC. NCCN guidelines : Non-Small Cell Lung Cancer (Version 7.2015). 2015.

[13] Chen Y, Guo W, Lu Y, Zou B. Dose-individualized stereotactic body radiotherapy for T1-3NO non-small cell lung cancer: long-term results and efficacy of adjuvant chemotherapy. Radiotherapy and oncology : journal of the European Society for Therapeutic Radiology and Oncology. 2008;88:351-8.

[14] Lambin P, Rios-Velazquez E, Leijenaar R, Carvalho S, van Stiphout RG, Granton P, et al. Radiomics: extracting more information from medical images using advanced feature analysis. European journal of cancer. 2012;48:441-6.

[15] Kumar V, Gu Y, Basu S, Berglund A, Eschrich SA, Schabath MB, et al. Radiomics: the process and the challenges. Magnetic resonance imaging. 2012;30:1234-48. 
[16] Leijenaar RT, Carvalho S, Velazquez ER, van Elmpt WJ, Parmar C, Hoekstra OS, et al. Stability of FDG-PET Radiomics features: an integrated analysis of test-retest and inter-observer variability. Acta oncologica. 2013;52:1391-7.

[17] Parmar C, Rios Velazquez E, Leijenaar R, Jermoumi M, Carvalho S, Mak RH, et al. Robust Radiomics feature quantification using semiautomatic volumetric segmentation. PloS one. 2014;9:e102107.

[18] Rios Velazquez E, Aerts HJ, Gu Y, Goldgof DB, De Ruysscher D, Dekker A, et al. A semiautomatic CT-based ensemble segmentation of lung tumors: comparison with oncologists' delineations and with the surgical specimen. Radiotherapy and oncology : journal of the European Society for Therapeutic Radiology and Oncology. 2012;105:167-73.

[19] Aerts HJ, Velazquez ER, Leijenaar RT, Parmar C, Grossmann P, Carvalho S, et al. Decoding tumour phenotype by noninvasive imaging using a quantitative radiomics approach. Nature communications. 2014;5:4006.

[20] Segal E, Sirlin CB, Ooi C, Adler AS, Gollub J, Chen X, et al. Decoding global gene expression programs in liver cancer by noninvasive imaging. Nature biotechnology. 2007;25:675-80.

[21] Zinn PO, Mahajan B, Sathyan P, Singh SK, Majumder S, Jolesz FA, et al. Radiogenomic mapping of edema/cellular invasion MRI-phenotypes in glioblastoma multiforme. PloS one. 2011;6:e25451.

[22] Coroller TP, Grossmann P, Hou Y, Rios Velazquez E, Leijenaar RT, Hermann G, et al. CT-based radiomic signature predicts distant metastasis in lung adenocarcinoma. Radiotherapy and oncology : journal of the European Society for Therapeutic Radiology and Oncology. 2015;114:345-50.

[23] Coroller TP, Agrawal V, Narayan V, Hou Y, Grossmann P, Lee SW, et al. Radiomic phenotype features predict pathological response in non-small cell lung cancer. Radiotherapy and oncology : journal of the European Society for Therapeutic Radiology and Oncology. 2016.

[24] Parmar CG, P.; Rietveld, D.; Rietbergen, M. M.; Lambin, P.; Aerts, H. J. Radiomic Machine Learning Classifiers for Prognostic Biomarkers of Head \& Neck Cancer. Front Oncol. 2015;5.

[25] Cunliffe A, Armato SG, 3rd, Castillo R, Pham N, Guerrero T, Al-Hallaq HA. Lung texture in serial thoracic computed tomography scans: correlation of radiomics-based features with radiation therapy dose and radiation pneumonitis development. International journal of radiation oncology, biology, physics. 2015;91:1048-56.

[26] Parmar C, Leijenaar RT, Grossmann P, Rios Velazquez E, Bussink J, Rietveld D, et al. Radiomic feature clusters and Prognostic Signatures specific for Lung and Head \&Neck cancer. Scientific reports. 2015; 5:11044.

[27] Kim H, Park CM, Goo JM, Wildberger JE, Kauczor HU. Quantitative Computed Tomography Imaging Biomarkers in the Diagnosis and Management of Lung Cancer. Investigative radiology. 2015;50:571-83.

[28] De Ruysscher D, Sharifi H, Defraene G, Kerns SL, Christiaens M, De Ruyck K, et al. Quantification of radiation-induced lung damage with $\mathrm{CT}$ scans: the possible benefit for radiogenomics. Acta oncologica. 2013;52:1405-10.

[29] Palma DA, van Sornsen de Koste J, Verbakel WF, Vincent A, Senan S. Lung density changes after stereotactic radiotherapy: a quantitative analysis in 50 patients. International journal of radiation oncology, biology, physics. 2011;81:974-8.

[30] Kyas I, Hof H, Debus J, Schlegel W, Karger CP. Prediction of radiation-induced changes in the lung after stereotactic body radiation therapy of non-small-cell lung cancer. International journal of radiation oncology, biology, physics. 2007;67:768-74.

[31] Mattonen SA, Palma DA, Haasbeek CJ, Senan S, Ward AD. Early prediction of tumor recurrence based on $\mathrm{CT}$ texture changes after stereotactic ablative radiotherapy (SABR) for lung cancer. Medical physics. 2014;41:033502.

[32] Defraene G, van Elmpt W, Crijns W, Slagmolen P, De Ruysscher D. CT characteristics allow identification of patient-specific susceptibility for radiation-induced lung damage. Radiotherapy and oncology : journal of the European Society for Therapeutic Radiology and Oncology. 2015.

[33] Pieper S. HM, Kikinis R. 3D SLICER. Proceedings of the 1st IEEE International Symposium on Biomedical Imaging: From Nano to Macro. 2004;1:632-5. 
[34] Zhao B, James LP, Moskowitz CS, Guo P, Ginsberg MS, Lefkowitz RA, et al. Evaluating variability in tumor measurements from same-day repeat CT scans of patients with non-small cell lung cancer. Radiology. 2009;252:263-72.

[35] Gamer ML, J.; Singh, I.F.P. irr: Various Coefficients of Interrater Reliability and Agreement. R package version 0.84. 2012.

[36] Husson FJ, J.; Le, S.; Mazet, J. FactoMineR: Multivariate Exploratory Data Analysis and Data Mining. R package version 1.31.3. 2015.

[37] Team RC. R: A language and environment for statistical computing. R Foundation for Statistical Computing. Vienna, Austria2015.

[38] Schroder MS, Culhane AC, Quackenbush J, Haibe-Kains B. survcomp: an R/Bioconductor package for performance assessment and comparison of survival models. Bioinformatics. 2011;27:3206-8.

[39] Haibe-Kains B, Desmedt C, Sotiriou C, Bontempi G. A comparative study of survival models for breast cancer prognostication based on microarray data: does a single gene beat them all? Bioinformatics. 2008;24:2200-8.

[40] Gentleman RC, Carey VJ, Bates DM, Bolstad B, Dettling M, Dudoit S, et al. Bioconductor: open software development for computational biology and bioinformatics. Genome biology. 2004;5:R80.

[41] Benjamini YH, Y. Controlling the false discovery rate: a practical and powerful approach to multiple testing. Journal of the Royal Statistical Society Series B 1995;57:289-300.

[42] Storey JD. The positive false discovery rate: a Bayesian interpretation and the q-value. Annals of statistics. 2003:2013-35.

[43] Therneau T. A Package for Survival Analysis in S. Version 2.38. 2015.

[44] Steck H, Krishnapuram B, Dehing-oberije C, Lambin P, Raykar VC. On ranking in survival analysis: Bounds on the concordance index. Advances in neural information processing systems2008. p. 1209-16.

[45] Harrell FE, Lee KL, Mark DB. Tutorial in biostatistics multivariable prognostic models: issues in developing models, evaluating assumptions and adequacy, and measuring and reducing errors. Statistics in medicine. 1996;15:361-87.

[46] Muren LP, Thwaites DI. The on-going quest for treatment precision and conformality in radiotherapy. Radiotherapy and oncology : journal of the European Society for Therapeutic Radiology and Oncology. 2013;109:337-41.

[47] Visbal AL, Leighl NB, Feld R, Shepherd FA. Adjuvant Chemotherapy for Early-Stage Non-small Cell Lung Cancer. Chest. 2005;128:2933-43.

[48] Lo A, Chernoff H, Zheng T, Lo SH. Why significant variables aren't automatically good predictors. Proceedings of the National Academy of Sciences of the United States of America. 2015;112:13892-7.

[49] Pan H, Simpson DR, Mell LK, Mundt AJ, Lawson JD. A survey of stereotactic body radiotherapy use in the United States. Cancer. 2011;117:4566-72. 


\section{Chapter}

\section{Associations of radiomic data extracted from static and respiratory-gated CT scans with disease recurrence in lung cancer patients treated with SBRT}




\section{ABSTRACT}

Radiomics aims to quantitatively capture the complex tumor phenotype contained in medical images to associate them with clinical outcomes. This study investigates the impact of different types of computed tomography (CT) images on the prognostic performance of radiomic features for disease recurrence in early stage non-small cell lung cancer (NSCLC) patients treated with stereotactic body radiation therapy (SBRT). 112 early stage NSCLC patients treated with SBRT that had static free breathing (FB) and average intensity projection (AIP) images were analyzed. Nineteen radiomic features were selected from each image type (FB or AIP) for analysis based on stability and variance. The selected FB and AIP radiomic feature sets had 6 common radiomic features between both image types and 13 unique features. The prognostic performances of the features for distant metastasis (DM) and locoregional recurrence (LRR) were evaluated using the concordance index $(\mathrm{Cl})$ and compared with two conventional features (tumor volume and maximum diameter). P-values were corrected for multiple testing using the false discovery rate procedure. None of the FB radiomic features were associated with DM, however, seven AIP radiomic features, that described tumor shape and heterogeneity, were ( $\mathrm{Cl}$ range: 0.638-0.676). Conventional features from FB images were not associated with DM, however, AIP conventional features were ( $\mathrm{Cl}$ range: 0.643-0.658). Radiomic and conventional multivariate models were compared between FB and AIP images using cross validation. The differences between the models were assessed using a permutation test. AIP radiomic multivariate models (median $\mathrm{Cl}=0.667$ ) outperformed all other models (median $\mathrm{Cl}$ range: $0.601-0.630$ ) in predicting DM. None of the imaging features were prognostic of LRR. Therefore, image type impacts the performance of radiomic models in their association with disease recurrence. AIP images contained more information than FB images that were associated with disease recurrence in early stage NSCLC patients treated with SBRT, which suggests that AIP images may potentially be more optimal for the development of an imaging biomarker. 


\section{INTRODUCTION}

Advances in science and technology have led to the understanding that each tumor, even within the same cancer type, has a myriad of distinct genotypic and phenotypic characteristics. This heterogeneity among tumors results in a spectrum of responses to treatments, and has led to the evolution of precision medicine [1]. In precision medicine, treatment plans are tailored towards the individual needs of each patient, largely based on their tumor characteristics and predicted therapeutic response, with the promise of improving overall survival and quality of life. The success of precision medicine relies on a means to capture the complexity and intrinsic properties of the tumor that is predictive of the most efficacious treatments. Radiomics is one method that aims to do this non-invasively by creating a quantitative portrayal of the tumor phenotype through the extraction of advanced imaging features from medical images [2-4]. These radiomic features describe the tumor phenotype through quantifying properties related to its shape, texture and image intensity, and have been predictive of clinical outcomes [5-15] and tumor characteristics, such as genotype and protein expression [16-18].

The majority of radiomics studies have focussed on investigating features extracted from a single image type. However, it is important to consider that the tumor phenotype and its behaviour may be uniquely captured in different types of images, even within the same imaging modality. For example, in radiation therapy treatment planning, computed tomography (CT) is the main imaging modality utilized, but different types of $\mathrm{CT}$ images are acquired to provide additional information for the treatment plan. Commonly, treatment plans are designed on static free breathing (FB) helical CT images, however, in cases where organ motion is a concern, such as with lung tumors, four-dimensional (4D) CT image datasets are also acquired. This is the case for early stage non-small cell lung cancer (NSCLC) patients that are treated with stereotactic body radiation therapy (SBRT) (Fig. 1a). FB scans can provide additional information for contouring normal tissue structures and alignment of the patient with the radiation field. The treatment course is planned on 4DCT images. The utilization of both types of CT scans is one factor that has contributed to the excellent survival and local control of NSCLC patients treated with SBRT [19-25].

In the field of radiomics, the impact of different types of images on the prognostic performance of radiomic models has not yet been thoroughly investigated. While previous studies have reported the influence of different image types, scanning parameters or reconstruction algorithms on the variation in feature values [26-29], not many studies have investigated the differences in prognostic performance of these radiomic features for clinical outcomes [30]. Early stage NSCLC patients treated with SBRT are an ideal cohort to evaluate the prognostic impact of different image types, as both FB and 4D CT scans are routinely acquired in the clinic for each patient. Furthermore, despite the successes of SBRT, 13-23\% of these patients still experience recurrent disease [19-25] 
and radiomics may have an important role in identifying which patients would be at highest risk of recurrence in order to adapt their course of treatment with the addition or intensification of therapy [31]. However, with the multiple types of CT images readily available for these patients, it is unknown which type of image would be optimal for radiomic analysis. The aim of this current study is to perform an initial exploratory evaluation of the prognostic performance of radiomic features extracted from FB and 4D CT scans to potentially identify which image type contains the most predictive radiomic information for disease recurrence in SBRT patients (Fig. 1B). Investigating the impact of image type on the prognostic performance of radiomic features is imperative for identifying the most optimal imaging biomarkers for precision medicine.

A)

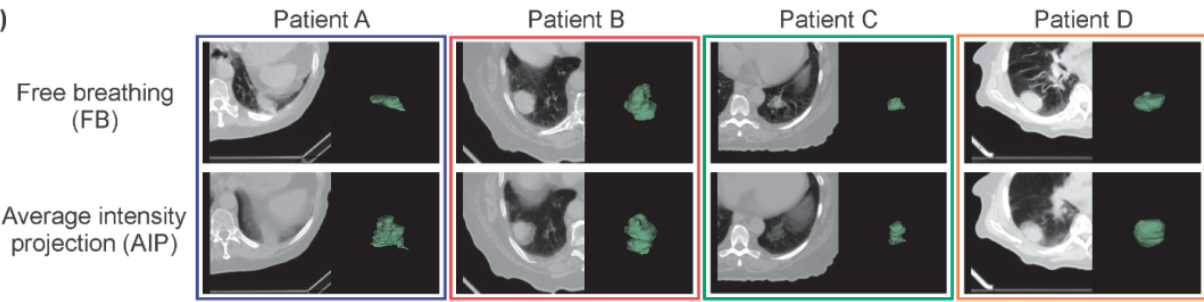

B) I. Tumor segmentation

II. Feature extraction
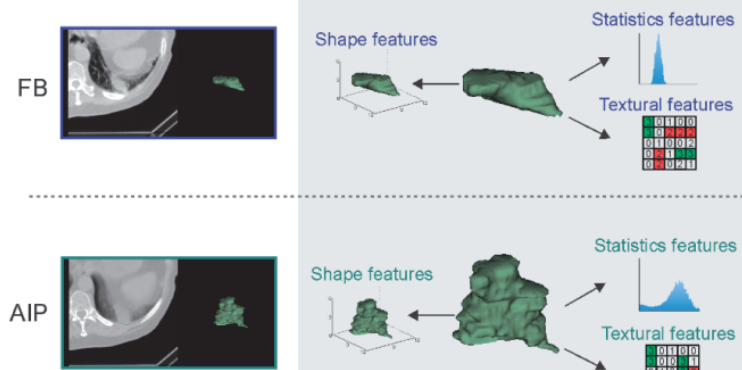

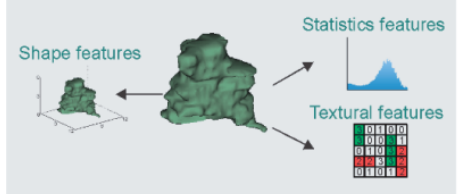

III. Feature selection

IV. Analysis

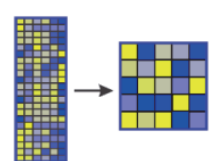

FB radiomic features

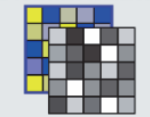

Clinical outcomes
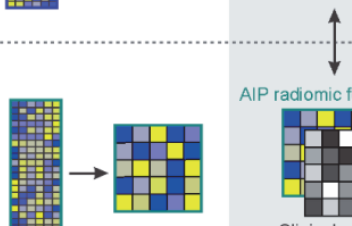

AlP radiomic features

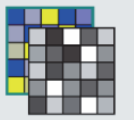

Clinical outcomes

Figure 1: A) Examples of free breathing (FB) and average intensity projection (AIP) images, demonstrating the observable differences in tumor phenotype between each image type. AIP images were reconstructed from 4D computed tomography (CT) scans. B) Schematic representation of the radiomics workflow for FB and AIP images. I. CT images of the patient are acquired and the tumor is segmented. II. Imaging features (radiomic and conventional features) are extracted from the tumor volume. III. Radiomic features undergo a feature dimension reduction process to generate a low-dimensional feature set based on feature stability and variance. IV. Imaging features are then analyzed with clinical outcomes to evaluate their prognostic power. FB and AIP radiomics features are compared. 


\section{MATERIALS AND METHODS}

\section{Patient characteristics}

One hundred and seventy patients with early stage NSCLC that were treated with SBRT at our institution from 2009-2014 were included in this study. This study was Institutional Review Board (IRB) approved by the Dana-Farber Cancer Institute IRB. Waiver of consent was approved for this retrospective study. From these 170 patients, patients were excluded if they fulfilled any of the following criteria: did not have a FB CT on file $(n=10)$, the duration between the FB scan and beginning of SBRT was greater than 1 week $(n=2)$, had multiple SBRT treatment courses and/or multiple primary tumors $(n=17)$, received induction chemotherapy $(n=2)$, had metastases to the lung from other primary sites $(n=30)$, locally recurrent disease $(n=5)$, small cell lung cancer $(n=1)$ or atypical carcinoid ( $n=1$ ) histology, or overall stage III or IV ( $n=1)$. Patients that did not complete the full course of treatment were also excluded $(n=1)$. After applying these exclusion criteria, 112 patients were included in the radiomics analysis. The patient, treatment and tumor characteristics are reported in Table 1. Details regarding SBRT treatment protocol and assessment of clinical outcomes can be found in S1 File.

\section{CT image acquisition and tumor segmentation}

All patients had both FB and 4D CT scans acquired on a GE LightSpeed RT16 CT scanner (GE Medical Systems, Milwaukee, WI, USA) according to standard clinical scanning protocols. The most common imaging slice thickness and pixel spacing was $2.5 \mathrm{~mm}$ and $1.27 \mathrm{~mm}$ by $1.27 \mathrm{~mm}$, respectively. All FB and AIP images were acquired with $120 \mathrm{kVp}$, and a standard reconstruction convolution kernel. AIP images were reconstructed from 4D CT image datasets that were acquired in axial cine mode, corresponding to one breathing cycle. The primary tumor site was manually contoured on FB and AIP images by E.H., V.A., and Y.H. on Eclipse software (Varian Medical Systems, Palo Alto, CA, USA), and then individually verified by an expert radiation oncologist (R.H.M.).

\section{Radiomic feature extraction}

A set of 644 radiomic features was extracted from tumor volumes isolated from FB or AIP images (Fig. 1B) using an in-house Matlab 2013 toolbox (The Mathworks Inc., Natick, MA, USA) and 3D Slicer 4.4 .0 software [32]. The intensities in the raw image were discretized using a bin width of 25 Hounsfield units for the texture features in order to increase sensitivity relative to the raw image, reduce image noise and normalize the intensities across all the patients. All CT voxels were resampled to $1 \times 1 \times 1 \mathrm{~mm}^{3}$ using a bicubic interpolation function prior to feature extraction. Radiomic features were categorized as shape, statistics or texture features. Shape features describe the three dimensional phys- 
ical appearances of the tumor, statistics features quantify properties of the voxel intensity histogram, and texture features quantify the spatial relationships between voxel intensities. A brief description of the selected radiomic features can be found in S Table 1 in the Supplementary Information and a full description of all the radiomic features can be found in the supplementary material from a previous study [5].

Radiomic features were compared against commonly used clinical CT metrics (referred to as "conventional features"), which included tumor volume and maximum diameter. The tumor diameter was calculated as the maximum diameter measured in a single axial imaging slice.

\section{Radiomic feature dimension reduction}

All feature selection and statistical analyses was conducted using $R$ software version 3.3.0 [33]. A two-step feature dimension reduction method was used to reduce the high-dimensional radiomic feature set to a low-dimensional radiomic feature set for analysis. First, stable features were selected using the test-retest Reference Image Database to Evaluate Therapy Response (RIDER) dataset [34]. The RIDER dataset consists of a series of CT images from 31 NSCLC patients obtained approximately 15 minutes apart in a similar position. 644 radiomic features were extracted from these images and were assessed for how well they correlated across the two sets of images by calculating the intraclass correlation coefficient (ICC) (using the "irr" package [35]). Features with an ICC greater than 0.8 were considered stable and selected for further analysis. This step reduced the number of features to 286 stable features.

Second, the set of stable features was further reduced to a set of features that would retain most of the variance within the data. Principal Component Analysis (PCA) and factor analysis was applied using the "FactoMineR" package [36]. Scores that retained $95 \%$ of the variability from the stable features and correlated by at least $99 \%$ to the PCA scores were selected. This resulted in 19 radiomic features for each image type (19 FB features, 19 AIP features). In total, 21 imaging features (19 radiomic and 2 conventional features) from each image type were investigated. The data for these features can be found in S2 File.

\section{Univariate data analysis}

The correlations between the imaging features from the FB and AIP images were evaluated using the Spearman's correlation coefficient $(\rho)$. FB and AIP features that had a $|\rho|$ $>0.8$ were considered to have a strong correlation.

The feature values were normalized (centred and scaled) into z-scores. The association between imaging features and clinical outcomes (i.e. the difference in feature values 
between patients with and without an event) was analyzed at the median time of event (10 and 9 months for DM and LRR, respectively) using a two-sided Wilcoxon rank-sum test. The difference in feature values was calculated by determining the difference in the median feature values for patients with and without the event. Patients that were censored or did not have an event before the considered time point were excluded in the assessment.

The concordance index $(\mathrm{Cl})[37,38]$ was used as a measure of the prognostic power of the imaging features for the clinical outcomes and was calculated using the "survcomp" package version 1.16 from Bioconductor [39]. The $\mathrm{Cl}$ is a generalization of the area under the receiver operating characteristic curve (AUC) that also incorporates time, and is a measure of the probability that between two randomly drawn samples, the sample with the higher value will have a higher probability of an event. $\mathrm{A} \mathrm{Cl}$ equal to 0.5 is equivalent to a random guess, greater than 0.5 indicates that the feature value is directly proportional to the probability of experiencing the outcome, and less than 0.5 indicates inverse proportionality (the lower the value, the higher the risk). P-values were computed using Noether's test to determine the significance of the $\mathrm{Cl}$ from random $(\mathrm{Cl}$ $=0.5$ ). Multiple testing correction was applied to all univariate results by the false discovery rate (FDR) procedure introduced by Benjamini and Hochberg [40], where pvalues less than 0.05 were considered statistically significant.

\section{Multivariate data analysis}

Five models for predicting DM were evaluated: FB conventional, FB radiomics, AIP conventional, AIP radiomics, and a combined FB and AIP radiomic model. A stratified cross validation approach was used, where the whole cohort was partitioned into training (80\%) and validation (20\%) datasets with matching event ratios. This stratified partitioning method was carried out 100 times, resulting in 100 different training (80\%) and validation (20\%) datasets. Multivariate models were trained on the training datasets and their performance was assessed in the corresponding validation dataset using concordance index $(\mathrm{Cl})$. In order to limit the number of features in each multivariate radiomics model, we used a lasso-based feature reduction method on the training dataset, which reduced the set of radiomic features to 5 features. The performance of each model was then assessed on the validation dataset. The performance between any two multivariate models was compared using a permutation test with 200 bootstrap iterations. P-values less than 0.05 were considered statistically significant. All the multivariate analysis was carried out using the R package "caret". 


\section{RESULTS}

The patient cohort included 112 early stage NSCLC patients that had been treated solely with SBRT. Patient and treatment characteristics are reported in Table 1 . The patient cohort had a median age of 74 (range: 47-89), was approximately equally split between genders (50.9\% female, $49.1 \%$ male), and predominantly Caucasian (91.1\%) and former smokers (73.2\%). All patients were overall stage I-II (NO, MO) and treated with SBRT with a median delivered biologically effective dose of 151.2 Gy (range: $100 \mathrm{~Gy}-151.2 \mathrm{~Gy}$ ). The median follow-up time was 20.8 months (range: $0.3-47.8$ months). $20.5 \%(n=23)$ of patients experienced DM with a median time to event of 10.0 months. $21.4 \%$ ( $n=$ 24) of patients developed LRR with a median time to event of 8.8 months. The 2-year estimates for DM and LRR were $74.0 \%$ and $70.9 \%$, respectively. All patients had both FB and AIP images acquired. The mean number of slices per tumor in the AIP images was 10.8 slices (range: 1.1-26.6).

Table 1. Patient, tumor, and treatment characteristics and clinical outcomes

\begin{tabular}{|c|c|c|}
\hline & & $\begin{array}{l}\text { Total ( } n=112 \text { patients) } \\
\text { median (range) or number (\%) }\end{array}$ \\
\hline \multicolumn{3}{|l|}{ Patient characteristics } \\
\hline Age & & $74(47-89)$ \\
\hline Gender & Female/ Male & $57 / 55(50.9 / 49.1)$ \\
\hline \multirow[t]{3}{*}{ Ethnicity } & African-American & $8(7.1)$ \\
\hline & Asian & $2(1.8)$ \\
\hline & Caucasian & $102(91.1)$ \\
\hline Smoking & Never/Current/ Former & $3 / 27 / 82(2.7 / 24.1 / 73.2)$ \\
\hline Pack-years & & $50(0.4-180.0)$ \\
\hline Performance status & $0 / 1 / 2 / 3$ & $\begin{array}{c}17 / 50 / 39 / 6(15.2 / 44.6 / \\
34.8 / 5.4)\end{array}$ \\
\hline \multicolumn{3}{|l|}{ Tumor characteristics } \\
\hline Overall stage & $\mid \mathrm{A} / \mathrm{IB} / \mathrm{II} \mathrm{A}$ & $94 / 17 / 1$ (83.9/ 15.2/ 0.9) \\
\hline T stage & $\mathrm{T} 1 \mathrm{a} / \mathrm{T} 1 \mathrm{~b} / \mathrm{T} 2 \mathrm{a} / \mathrm{T} 2 \mathrm{~b}$ & $\begin{array}{c}66 / 27 / 18 / 1(58.9 / 24.1 / 16.1 / \\
0.9)\end{array}$ \\
\hline \multirow[t]{5}{*}{ Histology } & Adenocarcinoma & $48(42.8)$ \\
\hline & Adenosquamous carcinoma & $1(0.9)$ \\
\hline & Squamous cell carcinoma & $27(24.1)$ \\
\hline & Undifferentiated NSCLC & $16(14.3)$ \\
\hline & No pathology & $20(17.9)$ \\
\hline \multicolumn{3}{|l|}{ Treatment characteristics } \\
\hline SBRT technique & 3D Conformal / VMAT & $84 / 28(75 / 25)$ \\
\hline Prescribed radiation dose (Gy) & & $54(48-60)$ \\
\hline Radiation dose per fraction (Gy) & & $18(10-18)$ \\
\hline Number of radiation fractions & $3 / 4 / 5$ & $67 / 2 / 43$ (59.8/ 1.8/ 38.4) \\
\hline \multicolumn{2}{|c|}{ Delivered biologically effective dose (Gy) } & $151.2(100-151.2)$ \\
\hline
\end{tabular}




\begin{tabular}{llc}
\hline & & $\begin{array}{c}\text { Total ( } \mathrm{n}=112 \text { patients) } \\
\text { median (range) or number (\%) }\end{array}$ \\
\hline $\begin{array}{l}\text { Clinical outcomes } \\
\text { Follow-up time (months) }\end{array}$ & $20.8(0.3-47.8)$ \\
Distant metastasis (DM) & No/ Yes & $89 / 23(79.5 / 20.5)$ \\
& Time to event (months) & $10.0(2.0-37.7)$ \\
Estimate of freedom from DM at & $74.0 \%$ \\
Locoregional recurrence (LRR) & No/ Yes & \\
& Time to event (months) & $88 / 24(78.6 / 21.4)$ \\
& Estimate of freedom from LRR at & $8.8(2.0-26.4)$ \\
2 years & $70.9 \%$ \\
No/ Yes & $53 / 59(47.3 / 52.7)$ \\
& Time to event (months) & $22.5(1.3-47.8)$ \\
& Estimate of survival at 2 years & $61.8 \%$ \\
\hline
\end{tabular}

Radiomic features were extracted from both FB and AIP images from each patient. Each feature set was reduced to 19 radiomic features, which were selected based on stability and maintaining the variance in the feature datasets. Two sets (FB and AIP) of 21 imaging features (2 conventional, 19 radiomic) were included in our analysis (Table 2 ). The two feature sets shared six common radiomic features, 2 features describing tumor shape, 3 features describing the intensity histogram or statistics of the tumor, and 1 feature describing the homogeneity of the tumor texture. The remaining 13 radiomic features in both the FB and AIP feature sets were unique to each image type. The unique radiomic features to the FB images were statistics features ( 6 features) or texture features ( 7 features). The unique radiomic features to the AIP images belonged to all feature groups: shape ( 1 feature), statistics ( 4 features) and texture ( 8 features).

The correlation between the FB and AIP imaging features was assessed using the Spearman's correlation coefficient $(\rho)$. The majority of the FB and AIP features were not strongly correlated (mean $\rho=0.0524$ ) (S1 Fig.), however, 12 pairs of FB and AIP features did have a very strong correlation ( $\rho>0.8$ ) (S2 Table). The FB and AIP features that were strongly correlated were shape ( 9 of the 12 pairs of correlated features) and texture features ( 3 of the 12 pairs). 
Table 2. Imaging features selected for analysis.

\begin{tabular}{|c|c|c|c|c|}
\hline & \multicolumn{2}{|c|}{ Feature Group } & \multicolumn{2}{|l|}{ Feature } \\
\hline \multirow{2}{*}{$\begin{array}{l}\text { Conventional } \\
\text { features }\end{array}$} & \multirow{2}{*}{\multicolumn{2}{|c|}{ Conventional }} & \multicolumn{2}{|l|}{ Volume } \\
\hline & & & \multicolumn{2}{|c|}{ Max diameter } \\
\hline \multirow{6}{*}{$\begin{array}{l}\text { Common } \\
\text { features } \\
\text { between FB and } \\
\text { AIP images }\end{array}$} & \multirow{2}{*}{\multicolumn{2}{|c|}{ Shape }} & \multicolumn{2}{|l|}{ Sphericity } \\
\hline & & & \multicolumn{2}{|c|}{ Sphere disproportionality } \\
\hline & \multicolumn{2}{|l|}{ Statistics } & \multicolumn{2}{|c|}{ Wv LLL max } \\
\hline & & & \multicolumn{2}{|c|}{ Wv HHL range } \\
\hline & & & \multicolumn{2}{|c|}{ LoG 3mm 2D skewness } \\
\hline & Texture & & \multicolumn{2}{|c|}{ LoG 3mm 3D GLCM homogeneity1 } \\
\hline \multirow{15}{*}{$\begin{array}{l}\text { Unique features } \\
\text { to FB and AIP } \\
\text { images }\end{array}$} & $\mathrm{FB}$ & & AIP & \\
\hline & $\begin{array}{l}\text { Feature } \\
\text { Group }\end{array}$ & Feature & $\begin{array}{l}\text { Feature } \\
\text { Group }\end{array}$ & Feature \\
\hline & \multirow[t]{6}{*}{ Statistics } & Wv HLL max & Shape & Compactness2 \\
\hline & & Wv LLH total energy & \multirow[t]{4}{*}{ Statistics } & LoG 3mm 3D skewness \\
\hline & & Wv LLH mean & & Wv HHL kurtosis \\
\hline & & Wv LHL skewness & & Wv LLH skewness \\
\hline & & Wv HLL var & & Wv HLL skewness \\
\hline & & Wv HLH min & \multirow[t]{8}{*}{ Texture } & LoG 3mm 3D GLCM infoCorr2 \\
\hline & \multirow[t]{7}{*}{ Texture } & Wv LHL GLCM correl1 & & GLCM correl1 \\
\hline & & Wv HLH GLCM correl1 & & LoG 3mm 3D GLCM correl1 \\
\hline & & Wv LLL GLCM infoCorr2 & & Wv LLL GLCM clusShade \\
\hline & & LoG 3mm 3D GLCM clusProm & & LoG 3mm 2D GLCM clus Prom \\
\hline & & $\begin{array}{l}\text { Wv HLH GLSZM high intensity } \\
\text { large area emphasis }\end{array}$ & & $\begin{array}{l}\text { Wv HLH RLGL low gray level run } \\
\text { emphasis }\end{array}$ \\
\hline & & LoG 3mm 2D GLCM clusShade & & $\begin{array}{l}\text { Wv LHH GLSZM large area } \\
\text { emphasis }\end{array}$ \\
\hline & & Wv LLL GLCM infoCorr1 & & Wv LHH GLCM correl1 \\
\hline
\end{tabular}

Labels: Wv = wavelet; LoG = Laplacian of Gaussian; L = low; $\mathrm{H}=$ high; GLCM = Gray-Level Co-occurrence Matrix; GLSZM = Gray-Level Size Zone Matrix; RLGL = Run Low Gray Level;

The association between the imaging feature values and clinical outcomes was investigated for LRR and DM (Fig. 2, S2 and S3 Figs.). None of the FB radiomic features were significantly associated with DM or LRR, however, one AIP radiomic feature describing the skewness of the intensity histogram (LoG 3mm 3D stats skewness), was significantly associated with LRR ( $p$-value $=0.018$ ). The difference between patients who had a LRR event versus those that did not was 0.51 , indicating that higher values of skewness in the AIP images were associated with having a LRR event. 


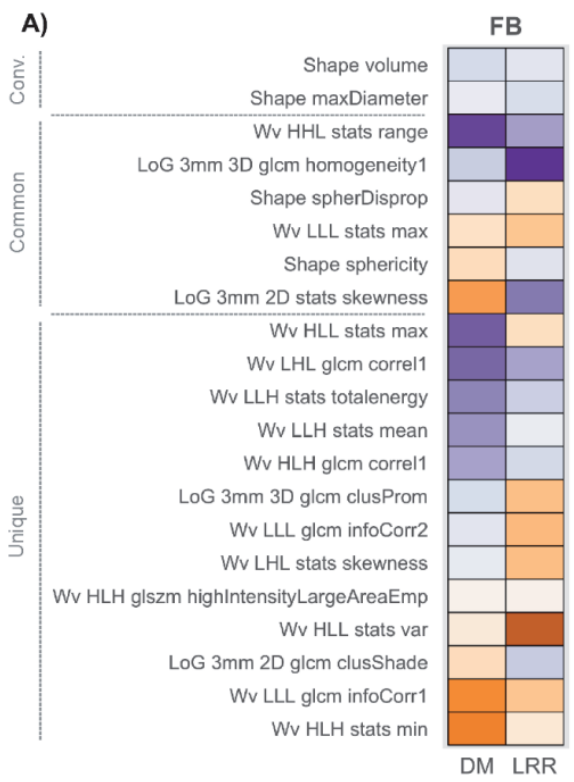

B)

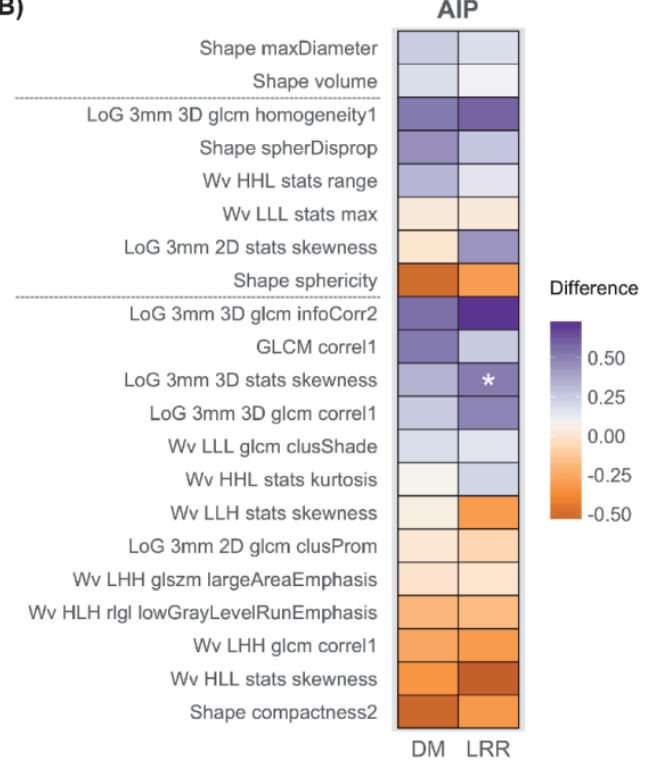

Figure 2. Heatmap of the association between imaging features and disease recurrence. Imaging features extracted from A) free breathing (FB) and B) average intensity projection (AIP) images were evaluated for their association with distant metastasis (DM) and locoregional recurrence (LRR). Features are grouped according to conventional (conv.) features, common features and unique features. "Common" features are radiomic features that had been selected from both FB and AIP images. "Unique" features are the radiomic features that were selected that are different between FB and AIP. The difference between the median values for each event status (event vs. no event) is plotted with the corresponding p-value indicated (Wilcoxon rank-sum test, FDR corrected $p$-values). The time point considered for DM and LRR was the median time of event (10 and 9 months for DM and LRR, respectively). * $p$-value $<0.05$.

The prognostic power of the FB and AIP imaging features was evaluated by calculating the concordance index $(\mathrm{Cl})$ for each feature (Fig. 3, S3 Table). None of the FB conventional or radiomic features from this particular dataset were prognostic of DM or LRR. However, features extracted from these AIP images were prognostic of DM. Both conventional features, maximum tumor diameter and volume, were prognostic of DM with $\mathrm{Cls}$ of 0.658 and 0.643 , respectively. Seven AIP radiomic features had Cls significantly greater than a random guess for DM that belonged to all feature groups: 3 texture features (Wavelet (Wv) High (H) Low (L)H Run Low Gray Level (RLGL), Low Gray Level Run emphasis (LGLRE), (Gray-Level Co-occurrence Matrix (GLCM) correl1 and Laplacian of Gaussian (LoG) 3mm 3D GLCM correl1, Cl range: 0.648 - 0.676), 1 statistics feature (WV $\mathrm{HLL}$ stats skewness, $\mathrm{Cl}=0.638$ ), and 3 shape features (compactness, sphericity and sphere disproportionality had $\mathrm{Cls}$ of 0.648 ). The corresponding $\mathrm{p}$-values for the significant features can be found in S3 Table. None of the AIP imaging features were prognostic of LRR. 
A)

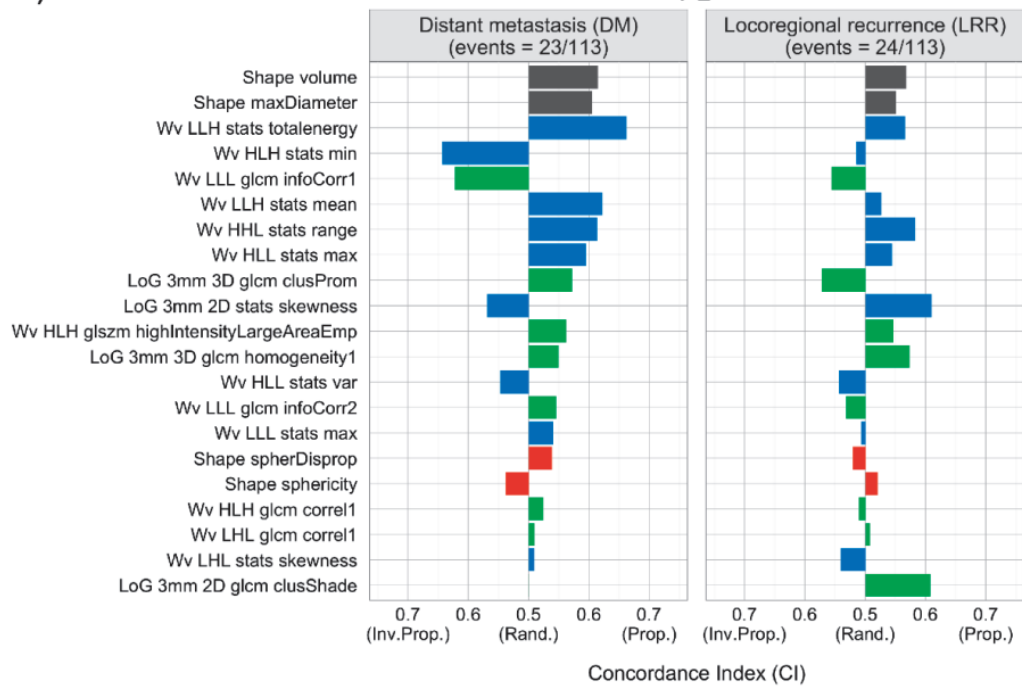

B)

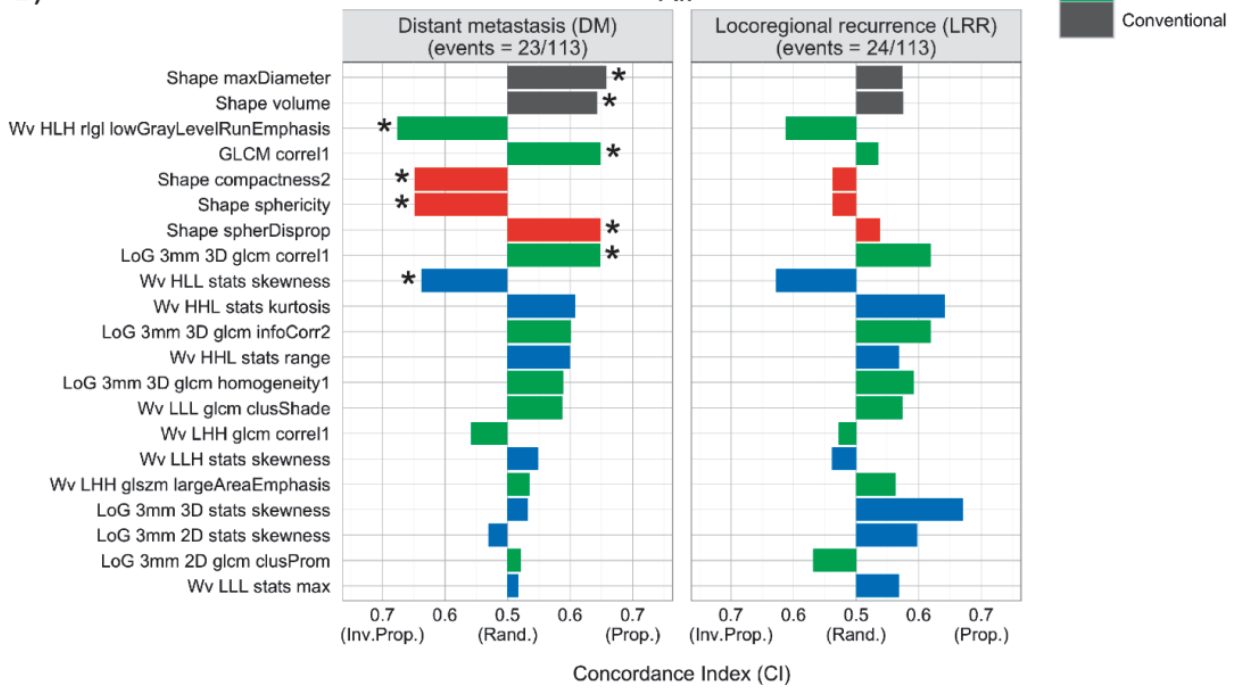

FB

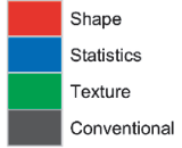

Figure 3. Prognostic performance of imaging features derived from A) FB or B) AIP images for disease recurrence in NSCLC patients treated with SBRT. Concordance indices $(\mathrm{Cl})$ are shown for each imaging feature and the clinical outcomes considered (distant metastasis (DM, left) and locoregional recurrence (LRR, right)). "Inv. Prop.", "Rand." and "Prop." indicate inversely proportional, equivalent to a random guess, and directly proportional, respectively. Conventional features are shown in grey and radiomic features are shown in red (shape), blue (statistics), and green (texture). ${ }^{*} p$-value $<0.05$ (Noether's test, FDR corrected p-values).

Multivariate models were generated for DM based on the imaging features from FB and AIP images using cross validation. Two imaging models were generated (conventional and radiomics) for each image type (FB or AIP) and a combined radiomics model (Fig. 4). 


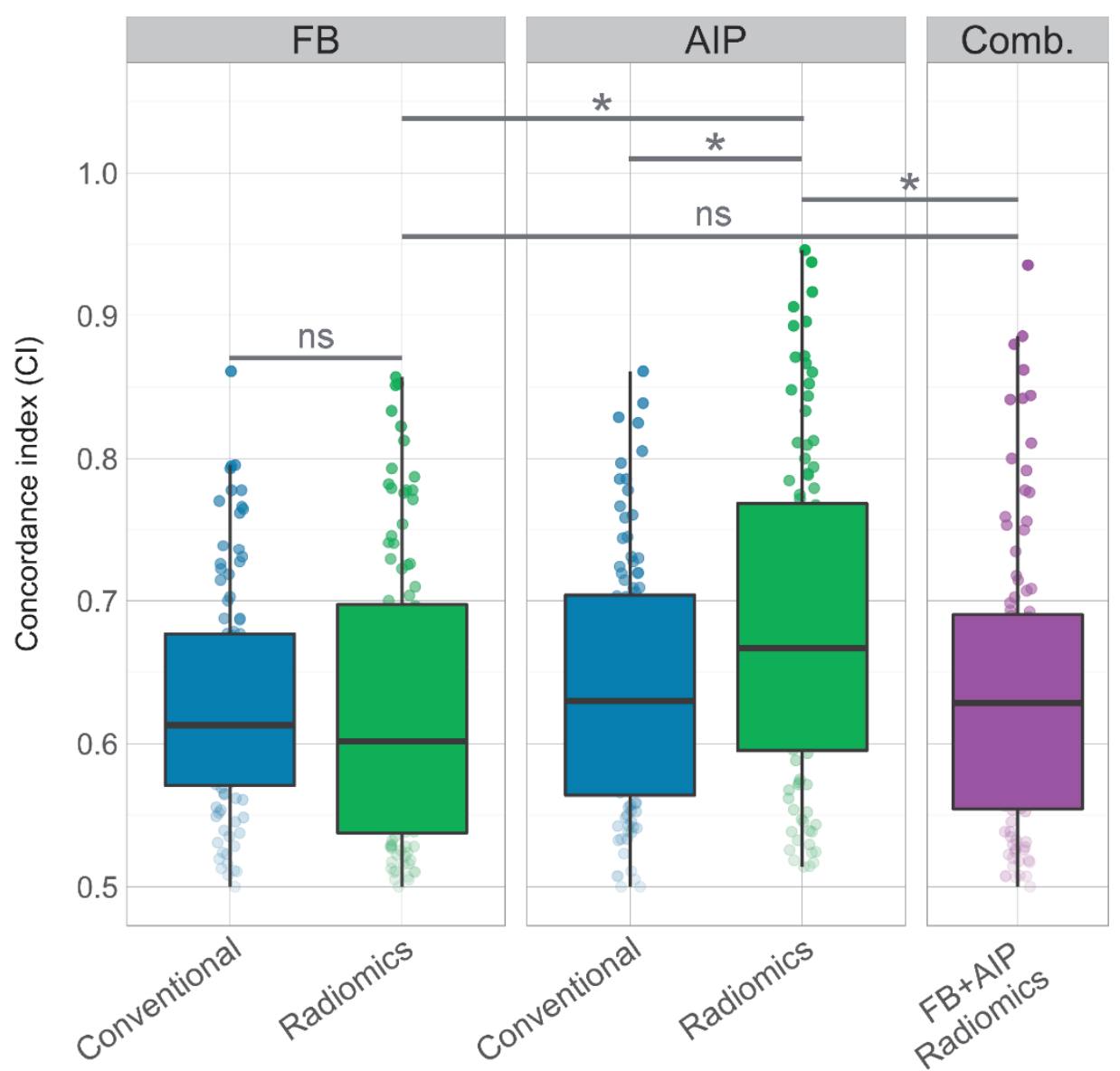

Figure 4. Performance of each multivariate model in predicting distant metastasis. Concordance indices are reported for the FB and AIP conventional and radiomic models, and a combined FB+AIP radiomics model, comparing the performance of each of model and image type. Cross validation was performed $(80 \%$ training, $20 \%$ validation) to generate 100 models for each model type. Comb. Indicates the combined FB and AIP radiomics model. *p-value $<0.05$; "ns" indicates not significant ( $p$-value $>0.05$ ).

The AIP radiomic model outperformed the $\mathrm{FB}$ radiomic model $(\mathrm{Cl}=0.667$ for $\mathrm{AIP}, \mathrm{Cl}=$ 0.601 for $F B, p$-value $=0.025)$ and the AIP conventional model $(\mathrm{Cl}=0.630, p$-value $=$ 0.045). However, the FB radiomics model performed similarly to the FB conventional model $(\mathrm{Cl}=0.613$, $\mathrm{p}$-value $=0.575)$. Combining the FB and AIP radiomic models did not increase the prognostic performance greater than the AIP radiomic model alone $(\mathrm{Cl}$ of combined model $=0.628$; -value $=0.01$ ). The selected features for the FB and AIP radiomic multivariate models were relatively stable across each repetition (S4 and S5 Figs.). The most commonly selected FB radiomic feature was WV HLH stats min, which was selected for 84 out of the 100 models. Wv HLH GLSZM High Intensity Large Area Em- 
phasis was chosen in 79 of the models and LoG 3mm 2D GLCM Clus Shade was chosen for 70 of the models. The most commonly selected AIP radiomic feature selected for multivariate AIP radiomic models was WV LLL GLCM ClusShade, which was selected in 99 of the models. Wv HLH RLGL Low Gray Level Run Emphasis (LGLRE) was also chosen in 79 of the models and Wv HHL stats kurtosis was chosen in 75 of the models.

\section{DISCUSSION}

Radiomics may have a critical role in precision medicine as it quantitatively describes, with great detail, the tumor phenotype captured in medical images by applying advanced mathematical algorithms to generate a high-dimensional atlas of imaging features. The type of image used for radiomic feature extraction impacts the feature values [26], and therefore, it is important to evaluate the impact of these variations in the features on their association with and potential ability to predict clinical outcomes. Thus far, studies investigating the effect of image type on the prognostic performance of radiomic features may have been limited due to a lack of comparable cohorts with the same patient and treatment characteristics, and clinical outcomes. Early stage NSCLC patients treated with SBRT have both FB helical and 4D CT scans acquired as the standard of care. Thus, this cohort provides a direct comparison of the impact of image type on the prognostic performance of radiomic features, where the clinical data is identical for both image types. Many different reconstructions of 4D CT scans can be investigated, however, we chose to use the AIP over other reconstructions such as the maximum intensity projection (MIP). Clinically, AIP images are used for radiation therapy treatment planning, while MIP images are used for contouring the internal target volume. The MIP images may have more artifacts because they capture the extremes of tumor motion, which may impact radiomic features to a greater extent than averaging the intensity, as in AIP images. For this reason, we chose to use AIP images over MIP images for this analysis.

Nineteen radiomic features were selected for analysis from FB and AIP images. Notably, 13 of the 19 features were different between FB and AIP images. These features were selected based on maintaining the variance in the dataset, and therefore, the difference in feature sets indicates that the images contain different radiomics information and the image type impacts the feature values. Only one AIP radiomics feature was associated with LRR, however, none of the conventional or radiomics features from FB or AIP images were prognostic for LRR. Furthermore, none of the imaging features were associated with DM, although several features were prognostic for DM in our dataset. This highlights the important notion that although a feature may be associated with a clinical outcome, it may not necessarily be prognostic since the properties of the feature distri- 
bution that qualify a feature as associative are not the same as the properties that qualify a feature as prognostic [41].

The significant AIP radiomic features describe different aspects of the tumor phenotype captured in the CT image. The AIP texture feature, LGLRE, describes the distribution of low gray level intensity values, where a lower feature value indicates fewer regions of low gray level intensities. In our dataset, the $\mathrm{Cl}$ was inversely proportional to the probability of an event, indicating that fewer regions of low gray level intensities were associated with a higher probability of developing DM. The AIP statistics feature, skewness, describes the shape of the voxel intensity histogram, where a lower skewness value indicates that the left tail (lower intensity values) of the intensity histogram is longer than the right tail (higher intensity values). Therefore, low skewness values indicate that there is a higher proportion of high intensity values, and in this dataset, is associated with a higher probability of DM. This directionality of the $\mathrm{Cl}$ for skewness provides a complementary interpretation of LGLRE, which also found that fewer regions of low intensity values were associated with a higher probability of DM. The texture features GLCM correl1 and LoG 3mm GLCM correl1 describe the correlation of the gray level cooccurrence matrix, where the latter feature has a LoG filter applied, which emphasizes the areas in the image with a rapid intensity change. Lastly, the three shape features describe how similar and dissimilar the tumor shape is to a sphere (sphericity and sphere disproportionality, respectively) and how closely packed together the tumor shape is (compactness). Tumors that were less spherical and less compact were associated with a higher probability of developing DM.

Importantly, in our particular dataset, radiomic features extracted from AIP images were prognostic of DM (had $\mathrm{Cl}$ significantly greater than 0.5 ), while radiomic features from FB images were not. This suggests that the tumor phenotype captured in FB and AIP images contain different prognostic information and the variation in feature values impacts the prognostic performance of the features. Importantly, despite having a strong correlation in FB and AIP images, the features describing the shape and dimensions of the tumor (shape radiomic features and conventional features) were prognostic in AIP images but not in FB. Furthermore, the three texture features (LGLRE and correl1) and one statistics feature (skewness) that were prognostic of DM in AIP images were not strongly correlated with any FB features. The difference in prognostic performance of the features between FB and AIP images highlights the impact of acquisition modes and reconstruction on the prognostic ability of the features since AIP images from 4D CT take into account organ motion, while FB images do not. Static FB CT scans represent a single snapshot of a dynamic lung tumor, and additionally, motion artifacts in FB scans can result in distortions of the tumor shape and compression of the tumor appearance in the image [42]. Furthermore, these artifacts impact the prognostic ability of the shape features in FB images since they may not be true depictions of the tumors. Motion artifacts are reduced in AIP images, which are reconstructions of multiple images 
across the breathing cycle, and thus, the tumors in these images may be a more accurate representation of the physical dimensions of the tumor, which may have a role in the prognostic ability of AIP shape features for DM.

Overall, we found that in our dataset, AIP images contained more prognostic radiomic features than FB images. This was also reflected in the multivariate analysis where radiomics models built from AIP radiomic features outperformed all other models (FB radiomic, and FB and AIP conventional). However, combining both FB and AIP radiomic models did not increase further increase their prognostic performance for DM, suggesting their radiomic information is not additive. Therefore, these results suggest that AIP images may be favoured over FB images for the development of imaging biomarkers for DM in NSCLC patients treated with SBRT, however, these particular conclusions pertain to our single dataset and requires further exploration and validation.

Previously, our group investigated the potential application of radiomics for lung cancer patients treated with SBRT using pre-treatment FB images and found that FB images did contain some prognostic information for predicting DM[9]. Comparatively, our current study uses a similar cohort of patients (112 patients in our current study vs. 113 patients previously), however the radiomics feature extraction and analysis is different. Our current study extracted a reduced set of radiomic features (644 features in the current study vs. 1605 features previously), which excluded many of the LoG features that were previously analyzed. Furthermore, we applied more stringent criteria for significance in our current study, where false discovery rate corrected $p$-values had a threshold of 0.05, whereas the previous studied applied a significance threshold of 0.1. Our previous study concluded that pre-treatment CT images may contain prognostic information for overall survival and DM. The current study elaborates on these findings and identifies that while FB images may contain prognostic information, the radiomics information found in AIP images may have stronger prognostic power than FB images and may potentially be a better option for developing an imaging biomarker for lung cancer patients treated with SBRT.

There are several limitations to our study that warrant discussion. SBRT is a fairly recently developed radiation therapy technique that is increasingly being adopted by more clinics and for more indications [43]. However, the recent implementation of SBRT limits the patient cohort size of our current study to only 113 patients that were treated between 2009 and 2014. As a result, our analytical methods were limited to an unsupervised feature selection method (not based on clinical outcomes) for radiomic feature dimension reduction and cross validation for the development and evaluation of multivariate models. However, we anticipate that over time, as clinical adoption of SBRT grows, a larger cohort of patients will be available for a validation study. Currently, SBRT is the standard of care for early stage medically inoperable NSCLC patients [44] and is being investigated as a non-invasive treatment option for operable early stage NSCLC 
patients [45]. Furthermore, the number of patients with early stage NSCLC is likely to increase due to lung screening efforts $[46,47]$, and thus, radiomics applied for SBRT patients has great potential to impact a rapidly growing patient population of early stage NSCLC patients. Thus, with the availability of a larger patient cohort, future studies will validate these findings in larger cohorts and a single radiomics signature may be developed as an imaging biomarker for early stage NSCLC patients treated with SBRT to predict the risk of DM from AIP images.

Another limitation of our study was that it was restricted to a single institution, and thus, a single image acquisition protocol for both FB and 4D CT scans. Future investigations will be required to confirm our findings across multiple institutions and with external training and validation datasets to evaluate the generalizability of our findings to all early stage NSCLC SBRT patients. Despite these limitations, our current study demonstrates that different image types contain varying degrees of prognostic radiomics information and it is important to consider the type of image used for radiomics analysis and development of an imaging biomarker.

This study investigated the performance of radiomic features extracted from FB and AIP CT images in evaluating their associations with disease recurrence that may be predictive of outcome in early stage NSCLC patients who had been treated with SBRT. In our particular dataset, AIP images contained more prognostic radiomic features than FB images, and multivariate models built from AIP radiomic features had the highest performance compared to FB radiomic and conventional models. This study emphasizes the importance of selecting the appropriate image type for radiomic analysis and identifies that even within the same imaging modality (e.g. CT), some types of images contain more prognostic information than others. As the field of radiomics continues to evolve in its applications in precision medicine, the selection of an optimal image type for analysis is highly important to develop the best performing imaging biomarkers for clinical outcomes. 


\section{REFERENCES}

1. Jameson JL, Longo DL (2015) Precision medicine--personalized, problematic, and promising. N Engl J Med 372: 2229-2234.

2. Lambin P, van Stiphout RG, Starmans MH, Rios-Velazquez E, Nalbantov G, et al. (2013) Predicting outcomes in radiation oncology--multifactorial decision support systems. Nat Rev Clin Oncol 10: 27-40.

3. Lambin P, Rios-Velazquez E, Leijenaar R, Carvalho S, van Stiphout RG, et al. (2012) Radiomics: extracting more information from medical images using advanced feature analysis. Eur J Cancer 48: 441-446.

4. Kumar V, Gu Y, Basu S, Berglund A, Eschrich SA, et al. (2012) Radiomics: the process and the challenges. Magn Reson Imaging 30: 1234-1248.

5. Coroller TP, Grossmann P, Hou Y, Rios Velazquez E, Leijenaar RT, et al. (2015) CT-based radiomic signature predicts distant metastasis in lung adenocarcinoma. Radiother Oncol 114: 345-350.

6. Yip SS, Coroller TP, Sanford NN, Huynh E, Mamon H, et al. (2016) Use of registration-based contour propagation in texture analysis for esophageal cancer pathologic response prediction. Phys Med Biol 61: 906-922.

7. Wu J, Aguilera T, Shultz D, Gudur M, Rubin DL, et al. (2016) Early-Stage Non-Small Cell Lung Cancer: Quantitative Imaging Characteristics of F Fluorodeoxyglucose PET/CT Allow Prediction of Distant Metastasis. Radiology: 151829.

8. Li H, Zhu Y, Burnside ES, Drukker K, Hoadley KA, et al. (2016) MR Imaging Radiomics Signatures for Predicting the Risk of Breast Cancer Recurrence as Given by Research Versions of MammaPrint, Oncotype DX, and PAM50 Gene Assays. Radiology: 152110.

9. Huynh E, Coroller TP, Narayan V, Agrawal V, Hou Y, et al. (2016) CT-based radiomic analysis of stereotactic body radiation therapy patients with lung cancer. Radiother Oncol.

10. Nie K, Shi L, Chen Q, Hu X, Jabbour S, et al. (2016) Rectal Cancer: Assessment of Neoadjuvant ChemoRadiation Outcome Based on Radiomics of Multi-Parametric MRI. Clin Cancer Res.

11. Huang YQ, Liang CH, He L, Tian J, Liang CS, et al. (2016) Development and Validation of a Radiomics Nomogram for Preoperative Prediction of Lymph Node Metastasis in Colorectal Cancer. J Clin Oncol 34: 2157-2164.

12. Coroller TP, Agrawal V, Narayan V, Hou Y, Grossmann P, et al. (2016) Radiomic phenotype features predict pathological response in non-small cell lung cancer. Radiother Oncol.

13. Parmar CG, P.; Rietveld, D.; Rietbergen, M. M.; Lambin, P.; Aerts, H. J. (2015) Radiomic Machine Learning Classifiers for Prognostic Biomarkers of Head \& Neck Cancer. Front Oncol 5.

14. Cunliffe A, Armato SG, 3rd, Castillo R, Pham N, Guerrero T, et al. (2015) Lung texture in serial thoracic computed tomography scans: correlation of radiomics-based features with radiation therapy dose and radiation pneumonitis development. Int J Radiat Oncol Biol Phys 91: 1048-1056.

15. Parmar C, Leijenaar RT, Grossmann P, Rios Velazquez E, Bussink J, et al. (2015) Radiomic feature clusters and Prognostic Signatures specific for Lung and Head \&Neck cancer. Sci Rep 5: 11044.

16. Aerts HJ, Velazquez ER, Leijenaar RT, Parmar C, Grossmann P, et al. (2014) Decoding tumour phenotype by noninvasive imaging using a quantitative radiomics approach. Nat Commun 5: 4006.

17. Segal E, Sirlin CB, Ooi C, Adler AS, Gollub J, et al. (2007) Decoding global gene expression programs in liver cancer by noninvasive imaging. Nat Biotechnol 25: 675-680.

18. Zinn PO, Mahajan B, Sathyan P, Singh SK, Majumder S, et al. (2011) Radiogenomic mapping of edema/cellular invasion MRI-phenotypes in glioblastoma multiforme. PLoS One 6: e25451.

19. Baumann P, Nyman J, Hoyer M, Wennberg B, Gagliardi G, et al. (2009) Outcome in a prospective phase II trial of medically inoperable stage I non-small-cell lung cancer patients treated with stereotactic body radiotherapy. J Clin Oncol 27: 3290-3296.

20. Chi A, Liao Z, Nguyen NP, Xu J, Stea B, et al. (2010) Systemic review of the patterns of failure following stereotactic body radiation therapy in early-stage non-small-cell lung cancer: clinical implications. Radiother Oncol 94: 1-11. 
21. Mak RH, Hermann G, Lewis JH, Aerts HJ, Baldini EH, et al. (2015) Outcomes by tumor histology and KRAS mutation status after lung stereotactic body radiation therapy for early-stage non-small-cell lung cancer. Clin Lung Cancer 16: 24-32.

22. Fakiris AJ, McGarry RC, Yiannoutsos CT, Papiez L, Williams M, et al. (2009) Stereotactic body radiation therapy for early-stage non-small-cell lung carcinoma: four-year results of a prospective phase II study. Int J Radiat Oncol Biol Phys 75: 677-682.

23. Timmerman R, Paulus R, Galvin J, Michalski J, Straube W, et al. (2010) Stereotactic body radiation therapy for inoperable early stage lung cancer. JAMA 303: 1070-1076.

24. Onishi H, Araki T, Shirato H, Nagata Y, Hiraoka M, et al. (2004) Stereotactic hypofractionated high-dose irradiation for stage I nonsmall cell lung carcinoma: clinical outcomes in 245 subjects in a Japanese multiinstitutional study. Cancer 101: 1623-1631.

25. Grills IS, Mangona VS, Welsh R, Chmielewski G, Mclnerney E, et al. (2010) Outcomes after stereotactic lung radiotherapy or wedge resection for stage I non-small-cell lung cancer. J Clin Oncol 28: 928-935.

26. Fave X, Cook M, Frederick A, Zhang L, Yang J, et al. (2015) Preliminary investigation into sources of uncertainty in quantitative imaging features. Comput Med Imaging Graph 44: 54-61.

27. Zhao B, Tan Y, Tsai WY, Qi J, Xie C, et al. (2016) Reproducibility of radiomics for deciphering tumor phenotype with imaging. Sci Rep 6: 23428.

28. Mackin D, Fave X, Zhang L, Fried D, Yang J, et al. (2015) Measuring Computed Tomography Scanner Variability of Radiomics Features. Invest Radiol 50: 757-765.

29. van Velden FH, Kramer GM, Frings V, Nissen IA, Mulder ER, et al. (2016) Repeatability of Radiomic Features in Non-Small-Cell Lung Cancer [F]FDG-PET/CT Studies: Impact of Reconstruction and Delineation. Mol Imaging Biol.

30. Cheng NM, Fang YH, Tsan DL, Hsu CH, Yen TC (2016) Respiration-Averaged CT for Attenuation Correction of PET Images - Impact on PET Texture Features in Non-Small Cell Lung Cancer Patients. PLoS One 11: e0150509.

31. Chen Y, Guo W, Lu Y, Zou B (2008) Dose-individualized stereotactic body radiotherapy for T1-3NO nonsmall cell lung cancer: long-term results and efficacy of adjuvant chemotherapy. Radiother Oncol 88: 351-358.

32. Pieper S. HM, Kikinis R. (2004) 3D SLICER. Proceedings of the 1st IEEE International Symposium on Biomedical Imaging: From Nano to Macro 1: 632-635.

33. Team RC (2015) R: A language and environment for statistical computing. R Foundation for Statistical Computing. Vienna, Austria.

34. Zhao B, James LP, Moskowitz CS, Guo P, Ginsberg MS, et al. (2009) Evaluating variability in tumor measurements from same-day repeat CT scans of patients with non-small cell lung cancer. Radiology 252: 263-272.

35. Gamer ML, J.; Singh, I.F.P. (2012) irr: Various Coefficients of Interrater Reliability and Agreement. R package version 0.84

36. Husson FJ, J.; Le, S.; Mazet, J. (2015) FactoMineR: Multivariate Exploratory Data Analysis and Data Mining. R package version 1.31.3.

37. Steck H, Krishnapuram B, Dehing-oberije C, Lambin P, Raykar VC. On ranking in survival analysis: Bounds on the concordance index; 2008. pp. 1209-1216.

38. Harrell FE, Lee KL, Mark DB (1996) Tutorial in biostatistics multivariable prognostic models: issues in developing models, evaluating assumptions and adequacy, and measuring and reducing errors. Statistics in medicine 15: 361-387.

39. Schroder MS, Culhane AC, Quackenbush J, Haibe-Kains B (2011) survcomp: an R/Bioconductor package for performance assessment and comparison of survival models. Bioinformatics 27: 3206-3208.

40. Benjamini YH, Y. (1995) Controlling the false discovery rate: a practical and powerful approach to multiple testing. Journal of the Royal Statistical Society Series B 57: 289-300.

41. Lo A, Chernoff H, Zheng T, Lo SH (2015) Why significant variables aren't automatically good predictors. Proc Natl Acad Sci U S A 112: 13892-13897. 


\section{Chapter 6}

42. Rietzel E, Pan T, Chen GT (2005) Four-dimensional computed tomography: image formation and clinical protocol. Med Phys 32: 874-889.

43. Pan H, Simpson DR, Mell LK, Mundt AJ, Lawson JD (2011) A survey of stereotactic body radiotherapy use in the United States. Cancer 117: 4566-4572.

44. Crino L, Weder W, van Meerbeeck J, Felip E, Group EGW (2010) Early stage and locally advanced (nonmetastatic) non-small-cell lung cancer: ESMO Clinical Practice Guidelines for diagnosis, treatment and follow-up. Ann Oncol 21 Suppl 5: v103-115.

45. Solda F, Lodge M, Ashley S, Whitington A, Goldstraw P, et al. (2013) Stereotactic radiotherapy (SABR) for the treatment of primary non-small cell lung cancer; systematic review and comparison with a surgical cohort. Radiother Oncol 109: 1-7.

46. Christensen JD, Chiles C (2015) Low-dose computed tomographic screening for lung cancer. Clin Chest Med 36: 147-160, vii.

47. National Lung Screening Trial Research T, Church TR, Black WC, Aberle DR, Berg CD, et al. (2013) Results of initial low-dose computed tomographic screening for lung cancer. N Engl J Med 368: 1980-1991. 


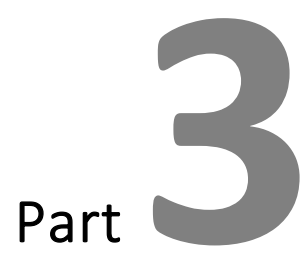

Tumor Intrinsic Nature Assessment 



\section{Chapter}

\section{Radiographic Prediction of Meningioma Grade by Semantic and Radiomic features}




\section{ABSTRACT}

The clinical management of meningioma is guided by tumor grade and biological behavior. Currently, the assessment of tumor grade follows surgical resection and histopathologic review. Reliable techniques for pre-operative determination of tumor grade may enhance clinical decision-making. A total of 175 meningioma patients (103 low-grade and 72 high-grade) with pre-operative contrast-enhanced T1-MRI were included. Fifteen radiomic (quantitative) and 10 semantic (qualitative) features were applied to quantify the imaging phenotype. The areas under the curve (AUC) and odds ratios (OR) were computed with multiple-hypothesis correction. Random forest classifiers were developed and validated on an independent dataset $(n=44)$. Twelve radiographic features (eight radiomic and four semantic) were significantly associated with meningioma grade. High-grade tumors exhibited necrosis/hemorrhage (ORsem=6.6, AUCrad=0.620.68), intratumoral heterogeneity (ORsem=7.9, AUCrad=0.65), non-spherical shape (AUCrad=0.61), and larger volumes (AUCrad=0.69) compared to low-grade tumors. Radiomic and sematic classifiers could significantly predict meningioma grade (AUCsem=0.71 and AUCrad=0.80). Furthermore, combining them increased the classification power (AUCradio=0.86). Clinical variables alone did not effectively predict tumor grade (AUCclin=0.59) or show complementary value with imaging data (AUC$\mathrm{comb}=0.84)$. We found a strong association between imaging features of meningioma and histopathologic grade, with ready application to clinical management. Combining qualitative and quantitative radiographic features significantly improved classification power. 


\section{INTRODUCTION}

Meningiomas are the most common primary brain tumor in adults, with most considered benign by the World Health Organization histopathologic criteria (WHO grade I) ${ }^{1,2}$. A distinct and increasing proportion of meningiomas are deemed high-grade (WHO grade II-III) and recur despite aggressive treatment, leading to substantial morbidity. Standard-of-care management typically involves surgical resection and often radiation therapy for high-grade (grade II-III) or progressive tumors.

Currently, the assessment of tumor grade occurs once a mass is resected and histopathological review is performed. Upon detection of a mass lesion that displays radiological features suggestive of meningioma, reliable parameters do not exist that can predict tumor grade and the associated clinical course. For example, clinical information such as age and gender show poor association with grade. Non-invasive and early predictors of meningioma grade may enhance clinical decision-making by providing prognostic information that could guide the decision of whether to observe or to treat.

The radiographic appearance of a tumor can be described using both quantitative and qualitative measures (Figure 1). Radiomics is an emerging field of quantitative imaging focused on leveraging large sets of imaging features to create an atlas ${ }^{3-5}$ that would foster the automatic, reproducible, and unbiased assessment of active clinical cases ${ }^{6-8}$. In comparison, semantic features are tumor traits (e.g. bone invasion, necrosis) that are assessed visually by radiologists. While semantic features are highly intuitive, they are inherently subject to inter-observer variability. Both radiomic ${ }^{9-18}$ and semantic ${ }^{19-22}$ features have been applied as prognostic biological signatures, and therefore, may offer complementary streams to predict clinical status.

In this study, we investigated the value of radiomic and semantic imaging features for predicting the histologic grade of meningiomas from preoperative gadoliniumenhanced T1-weighted MRI. 


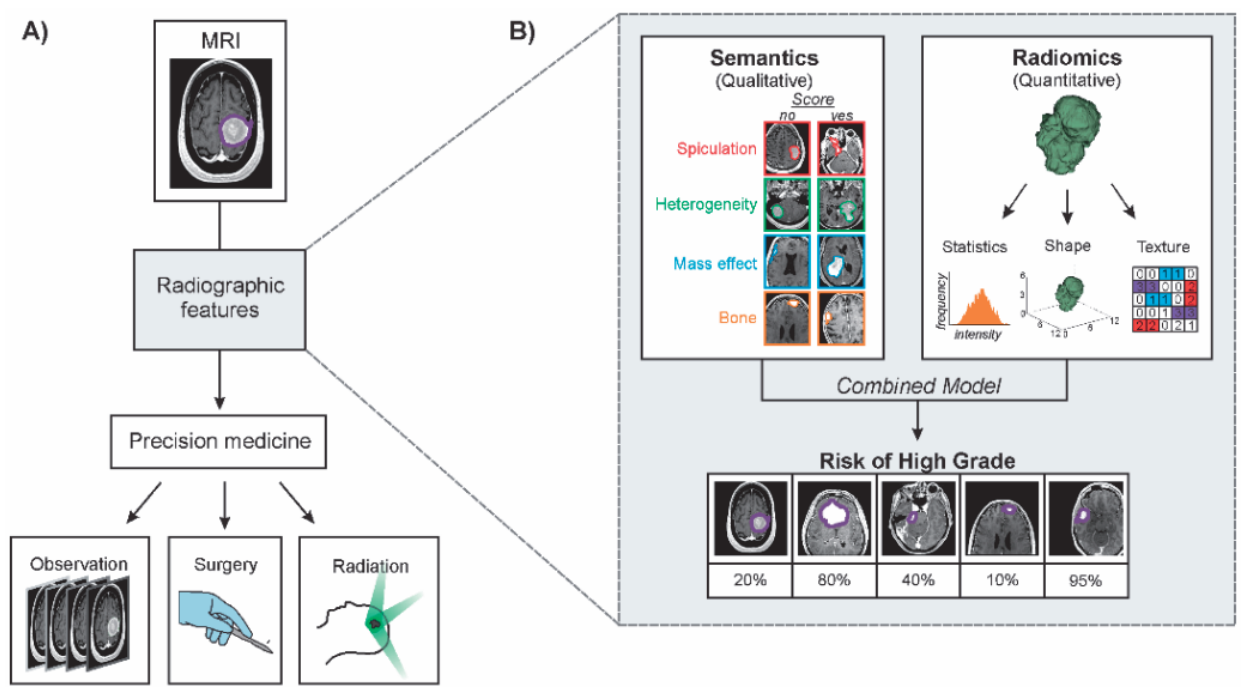

Figure 1. A) Potential impact of radiographic features on meningioma patient management. Pre-operative radiographic assessment of grade may improve the ability to tailor precision medicine decision trees to individual patients. B) A combined model of semantic and radiomic radiographic features was used to predict meningioma grade and validated on an independent cohort of meningiomas.

\section{METHODS}

This study was reviewed and approved by the human subjects institutional review boards (IRBs) of the Dana-Farber Cancer Institute and the Brigham and Women's Hospital (BWH). Patient consent was waived by IRB protocol. All methods were performed in accordance with the relevant guidelines and regulations.

\section{Patient data}

A total of 181 meningiomas resected at the Brigham and Women's Hospital (BWH) between 2003 and 2014 were reviewed for histopathology and imaging. Pre-operative gadolinium-enhanced T1-weighted MRI sequences were chosen for analysis to represent the most frequently reviewed images for meningiomas. Six cases with motion artifact were excluded from analyses.

Histopathologic review of all tumors was performed by two board-certified neuropathologists (S.S., M.A.). Meningiomas were graded according to the World Health Organization (WHO) classification system1. In this study, low and high grade refers to grade I and grade II/III, respectively. Atypical features for meningiomas were individually tabulated23. 


\section{Image-based Phenotyping}

In this study, semantic (qualitative) and radiomic (quantitative) feature quantification was applied to preoperative MRI (Figure 1B, Table 1). The standard BWH preoperative imaging protocol for intracranial tumors include a high-resolution gadolinium-enhanced T1-weighted 3D MPRAGE or SPGR sequence, acquired on a 1.5T or 3T scanner. For patients who had had serial imaging prior to surgery, we analyzed the MRI that was acquired closest to the date of surgery. We exported images into 3D Slicer24 for editing and reconstructed meningioma volumes from the manual contours of individual axial MRI slices performed by two neurosurgeons. All contours were reviewed by an experienced neuroradiologist (R.H.) to ensure standardization of contouring criteria across the dataset. We applied image processing prior to feature extraction to reduce noise (mean +/- 3 standard deviations) according to well-established MRI-normalization methods. We resampled the voxel dimensions using $3 \times 3 \times 3 \mathrm{~mm} 3$ as the common spacing.

Table 1. Description of radiographic features and filters. Individual descriptions are given for each group and parameter or feature.

\begin{tabular}{|c|c|c|c|}
\hline Type & Group & Feature / Parameter & Description \\
\hline \multirow[t]{19}{*}{$\begin{array}{l}\text { Radiographic } \\
\text { features }\end{array}$} & \multirow[t]{10}{*}{ Semantic } & \multicolumn{2}{|c|}{$\begin{array}{c}\text { Intratumoral heterogeneityHeterogeneity in hyperintensity of MRI signal } \\
\text { throughout tumor }\end{array}$} \\
\hline & & Multifocality & Non-contiguous growth of tumor \\
\hline & & Midline shift & Shift of the brain past midline \\
\hline & & Sinus invasion & Presence of venous sinus invasion \\
\hline & & Necrosis / Hemorrhage & Presence of necrosis or hemorrhage \\
\hline & & Mass effect & Shift in normal brain parenchyma due to tumor \\
\hline & & Cystic component & Fluid filled cysts within the tumor \\
\hline & & Bone invasion & Appearance of tumor invading the skull \\
\hline & & Hyperostosis & Bony overgrowth adjacent to tumor \\
\hline & & Spiculation & Irregularities in tumor shape and border \\
\hline & \multirow[t]{9}{*}{ Radiomic } & Median & Median voxel intensity value \\
\hline & & Mean & Mean voxel intensity value \\
\hline & & Minimum & Minimal voxel intensity value \\
\hline & & Skewness & $\begin{array}{l}\text { Describes the shape of a probability distribution of } \\
\text { the voxel intensity histogram }\end{array}$ \\
\hline & & $\begin{array}{l}\text { Spherical Disproportion } \\
\text { (SD) }\end{array}$ & $\begin{array}{l}\text { How different is the tumor is to a sphere with a } \\
\text { similar volume }\end{array}$ \\
\hline & & Cluster Prominence (CP) & Sensitive to flat zones (area of similar intensity) \\
\hline & & Difference Entropy (DE) & $\begin{array}{l}\text { Complexity of the pattern (high entropy for high } \\
\text { number of unique patterns) }\end{array}$ \\
\hline & & $\begin{array}{l}\text { Inverse Difference } \\
\text { Normalized (IDN) }\end{array}$ & Sensitive to homogeneity in the tumor \\
\hline & & $\begin{array}{l}\text { Run Length Non- } \\
\text { uniformity (RLN) }\end{array}$ & Measure of heterogeneity \\
\hline
\end{tabular}




\begin{tabular}{|c|c|c|c|}
\hline Type & Group & Feature / Parameter & Description \\
\hline & & $\begin{array}{l}\text { Short Run Low Gray-Level } \\
\text { Emphasis (SRLGLE) }\end{array}$ & $\begin{array}{l}\text { Measure of heterogeneity sensitive to low intensity } \\
\text { pattern }\end{array}$ \\
\hline & & $\begin{array}{l}\text { High Intensity Large Area } \\
\text { Emphasis (HILAE) }\end{array}$ & $\begin{array}{l}\text { Sensitive to flat zones with high intensity voxels } \\
\text { (e.g. areas of hemorrhage) }\end{array}$ \\
\hline & & $\begin{array}{l}\text { Low Intensity Large Area } \\
\text { Emphasis (LILAE) }\end{array}$ & $\begin{array}{l}\text { Sensitive to flat zones with low intensity voxels (e.g. } \\
\text { areas of necrosis) }\end{array}$ \\
\hline & & $\begin{array}{l}\text { Low Intensity Small Area } \\
\text { Emphasis (LISAE) }\end{array}$ & $\begin{array}{l}\text { Sensitive to small flat zones with low intensity } \\
\text { voxels }\end{array}$ \\
\hline \multirow[t]{2}{*}{ Filters } & Wavelet & High (L), Low (L) & $\begin{array}{l}\text { Wavelet filters decompose images by high (increase } \\
\text { details) and low (smooth image, leaving general } \\
\text { shape) for every spatial component }(x, y, z)\end{array}$ \\
\hline & LoG & Sigma $(\sigma)$ & $\begin{array}{l}\text { Laplacian of Gaussian is a filter that highlights } \\
\text { textures using a variable size radius ( } \sigma \text { ). Depending } \\
\text { on the radius (from } 0.5 \mathrm{~mm} \text { to } 5 \mathrm{~mm} \text { with } 0.5 \\
\text { increment), it emphasizes image textures from fine } \\
\text { to coarse. }\end{array}$ \\
\hline
\end{tabular}

Semantic features such as speculation and mass effect are MRI characteristics regularly assessed during the standard evaluation of images from patients with meningiomas. Ten semantic features were scored ( 0 or 1 ) by an experienced neuroradiologist (R.H.) whereas radiomic features were extracted from images using a custom Matlab script. A total of 1,055 radiomic features were computed that quantify the tumor phenotype (description in Supplement I). We selected fifteen features for this study based on their variance and correlation. Additionally, we included two tumor size features (maximum axial diameter and volume) in the clinical data set, along with age, gender, and radiation induced status.

\section{Univariate analysis}

All statistical analyses were performed in R software version 3.3.125. Our primary endpoint was the potential applicability of radiographic features to predict meningioma grade. The predictive power of semantic features (binary) was evaluated using the odds ratio (OR) and Fisher's exact test. The predictive power of radiomic features (continuous) was analyzed using the area under the receiver operator characteristics curve (AUC) using the "survcomp" package26 and Noether's test.

Additionally, prediction of low grade (grade I) with the presence of atypical features was studied. A subset of the cohort with only low grade meningioma was analyzed, where we compared patients with one or more atypical features (including spontaneous necrosis, high nuclear-tocytoplasmic ratio, prominent nucleoli, and sheetlike growth) versus none of these features using the same imaging features as for grade prediction. Hypercellularity was almost ubiquitously observed across the meningioma cohort, and therefore, not included as an atypical feature for purposes of analysis. 
Finally, the association between radiomic and semantic features was investigated using the AUC. Every semantic feature was predicted by each of the radiomic features in a univariate manner. All p-values were adjusted for multiple hypothesis testing using the false discovery rate method 27 .

\section{Multivariate analysis}

A temporal split was used to assign patients to a training or validation dataset. Feature selection was based on the training dataset, to ensure independence from the validation dataset (Supplement I). Differences in clinical variables between datasets were assessed using the Fisher's exact test (for categorical variables) and the Wilcoxon test (for continuous variables).

We investigated five models for grade classification including 1) clinical, 2) semantic, 3) radiomic, 4) radiographic (combined radiomic and semantic features) and 5) combined radiographic and clinical. Classifications were made using the random forest method from the "randomForest" package28. Nested cross validation was used for model tuning and training using the "caret" package29 on the training set, leaving the validation dataset independent from the model selection process. Differences in predictive power between models were assessed using bootstrapping (1,000 iterations).

\section{RESULTS}

\section{Clinical cohort}

Our cohort of 175 patients was mainly composed of female patients (62\%), with a median age of 57 years (Table 2). 59\% of cases were low-grade and $41 \%$ were high-grade. No differences were observed in WHO grade $(p=0.48)$, radiation-induced status $(p=0.51)$, or gender $(p=0.15)$ between the training and validation datasets.

Table 2. Demographic information across the full, training, and validation datasets.

\begin{tabular}{llllll}
\hline Variable & Groups & $\begin{array}{l}\text { Full } \\
(\mathrm{n}=175)\end{array}$ & $\begin{array}{l}\text { Training } \\
(\mathrm{n}=131)\end{array}$ & $\begin{array}{l}\text { Validation } \\
(\mathrm{n}=44)\end{array}$ & p-value \\
\hline Age (years) & Median (range) & $57(22-89)$ & $57(22-89)$ & $57.5(29-89)$ & 0.28 \\
Gender & Male & $68(38 \%)$ & $55(42 \%)$ & $13(30 \%)$ & 0.15 \\
& Female & $107(62 \%)$ & $76(58 \%)$ & $31(70 \%)$ & 0.48 \\
WHO grade & Low (grade I) & $103(59 \%)$ & $75(57 \%)$ & $28(63 \%)$ & $16(37 \%)$ \\
Radiation-induced & High (grade II/III) & $72(41 \%)$ & $56(43 \%)$ & $2(4.5 \%)$ & 0.51 \\
& Yes & $13(7 \%)$ & $11(8.3 \%)$ & $42(95.5 \%)$ & \\
\hline
\end{tabular}




\section{Radiographic associations with meningioma grade or atypical features}

First, examination of individual semantic (qualitative) features revealed significant associations between meningioma grade and four features (Figure 2A.1, Table S2). These features included intratumoral heterogeneity $(O R=7.9, p<0.001)$, necrosis/hemorrhage $(\mathrm{OR}=6.6, \mathrm{p}=0.01)$, venous sinus invasion $(\mathrm{OR}=2.9, \mathrm{p}=0.02)$, and mass effect $(\mathrm{OR}=2.3$, $\mathrm{p}=0.042$ ). Interestingly, cystic component was not significantly associated with grade despite a high OR $(6.8, p=0.13)$, which is likely due a low incidence of events (6 cases, $3.4 \%)$, which introduces a high margin error. All significant features had an OR greater than one, indicating that higher grade corresponds to an increased incidence of the feature.

Second, we investigated the relationship between radiomic (quantitative) features and meningioma pathology (Figure 2A.2, Table S3). Eight radiomic features were significant from random in their association with tumor grade (range $A \cup C=0.59$ to $0.65, p<0.05$ ). The best performing radiomic feature, high intensity large area emphasis (HILAE), was associated with high grade meningioma ( $A \cup C=0.69, p<0.001$ ). HILAE is sensitive to large zones with high intensities (e.g. hemorrhage). In addition, low intensity large area emphasis (LILAE) was also associated with high grade meningioma ( $A U C=0.63, p=0.008$ ) and is sensitive to large areas of low intensities (e.g. necrosis). These suggest that hemorrhagic or necrotic tumors were more likely to be high grade, consistent with the semantic feature analysis. High values of spherical disproportion (SD), which measures the degree of deviation of a tumor's shape from a sphere of similar volume, and run length nonuniformity (RLN), which is sensitive to heterogeneity, were both significantly associated with high-grade tumors ( $A \cup C=0.61, p=0.012$ and $A \cup C=0.65, p=0.002$, respectively).

Additionally, we examined the ability of imaging to distinguish low grade meningiomas with ( $n=69)$ and without ( $n=34)$ one of four atypical features (Figure 2A, Tables S4 \& S5). While intratumoral heterogeneity and multifocality carried an OR of 1.7 and 2.1, respectively, no significant association was observed between semantic features and the presence of atypical features. In comparison, four radiomic features were significantly associated with atypical features. These features included voxel mean intensity ( $A \cup C=0.68$ ), low intensity small area emphasis (LISAE) (AUC=0.66), difference entropy (DE) $(A \cup C=0.66)$, and cluster prominence $(C P)(A \cup C=0.65)$. LISAE indicated that hypointense tumors were more likely to be low grade with atypical features. None of the low grade meningiomas had a cystic component; therefore, this semantic feature was not investigated in this analysis. 


\section{A) Prediction of Grade and Atypical Low Grade}

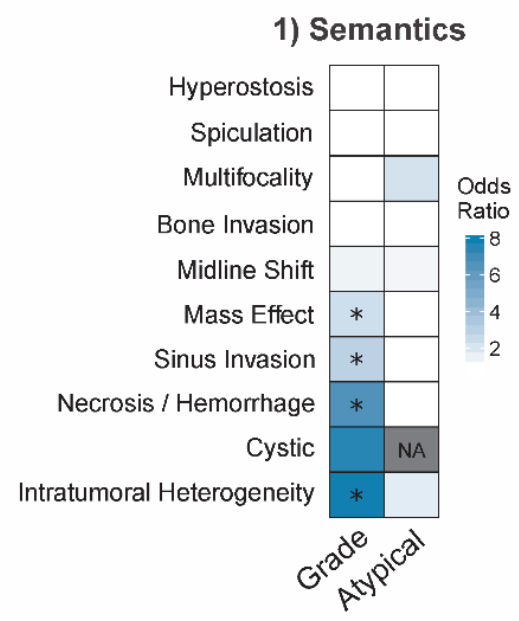

2) Radiomics

HHL Skewness

HLH Median

LoG5 Low Intensity Small Area Emp.

LLH Short Run Low Gray Level Emp.

Difference Entropy

$\mathrm{HHH}$ Mean

LoG4 High Intensity Large Area Emp.

HLL Cluster Prominence

HLH Mean

Spherical Disproportion

LoG5 Inv. Diff. Normalized

LHL Low Intensity Large Area Emp.

Run Length Non-Uniformity

Minimum

$\mathrm{HHH}$ High Intensity Large Area Emp.

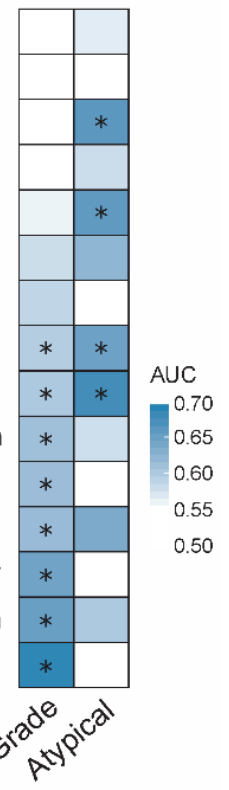

\section{B) Associations between semantic and radiomic features}

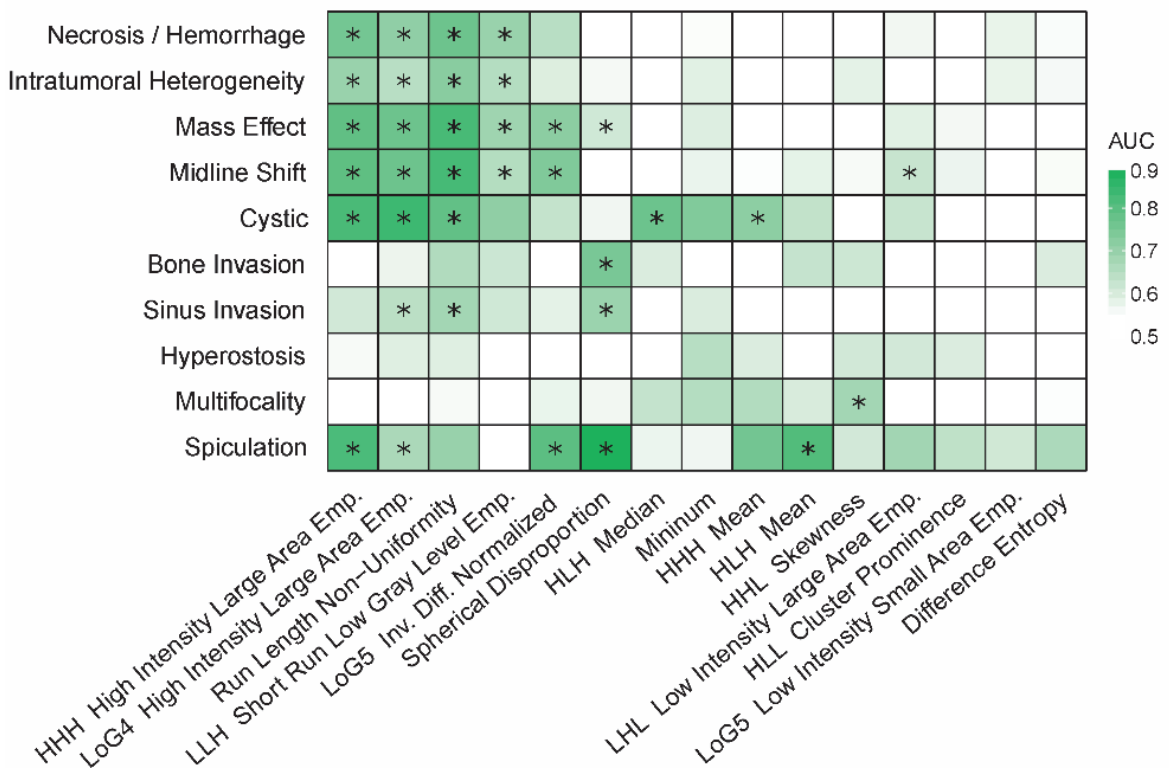

Figure 2. A) Heatmap of the predictive power of (1) semantic and (2) radiomic features for meningioma grade $(n=175)$ or presence of histopathologic atypia in low grade meningiomas $(n=103)$. B) The association between semantic and radiomic features was investigated. Every semantic feature was predicted with each of the radiomic feature in a univariate manner that indicates their relationship. * indicates significance from random after multiple correction. 
We investigated the link between radiomic and semantic features. We found a median AUC of 0.57 (range: 0.50-0.89) between these two categories of features (Figure 2.B, Table S6). A significant interaction between particular pairs of features was found $(p<0.05)$. Spherical disproportion (SD) was associated with mass effect ( $A \cup C=0.61$ ), spiculation ( $A \cup C=0.89$ ), and invasion of bone and venous sinus ( $A \cup C$ of 0.74 and 0.69 , respectively). Textural features, such as high intensity large area emphasis (HILAE), run length non-uniformity (RLN) and short run length gray-level (SRLGL) were associated with tumor heterogeneity ( $A \cup C=0.65-0.72$ ), cystic component ( $A \cup C=0.71-0.84)$, and hemorrhage / necrosis ( $A \cup C=0.70-0.76)$.

\section{Improving grade classification by combining radiographic features}

Given that radiomic and semantic analyses each provide a distinct quantification of the tumor phenotype, we explored whether combining radiomic and semantic features may be synergistic in predicting meningioma grade (Figure 3, Table S7).

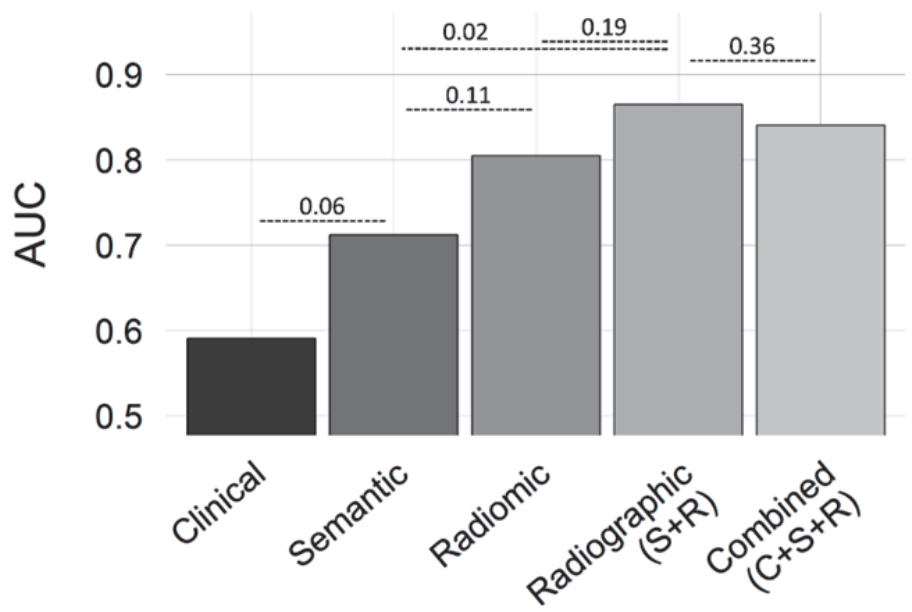

Figure 3. Area under the curve (AUC) from random forest models on the independent validation set $(n=44)$ for meningioma grade classification. P-values of significance between models are shown.

A model based on clinical data, composed of information available to a clinician prior to $M R$ imaging, did not associate with meningioma grade ( $A \cup C=0.59, p=0.32$ ). In comparison, both semantic ( $A \cup C=0.71$ ) and radiomic ( $A \cup C=0.80$ ) models independently classified meningioma grade $(p<0.05)$. Interestingly, a radiographic model that combined both radiomic and semantic features showed an increased performance in the classification of tumor grade (AUC=0.86, $\mathrm{p}<0.001)$. 
Although both radiomic and semantic models were individually significant, the classification performance of the radiomic model was significantly higher than the semantic $(p=0.02)$. Moreover, the combined radiographic model performed significantly better than all other models $(p<0.05)$ except for the radiomic model alone $(p=0.19)$. Lastly, adding clinical data to the radiographic model did not improve the performance $(A \cup C=0.84)$ compared to radiographic features alone $(p=0.36)$.

\section{DISCUSSION}

Meningioma grade is a powerful predictor of clinical outcome and therefore influences patient management, including the decision of whether to observe, operate, or administer adjuvant therapies. Currently, tumor grade can only be determined following surgical resection and histopathological review30. A better approach would allow clinicians to discriminate low and high grade meningiomas before surgery, thereby facilitating management decisions and counseling at an earlier stage of clinical care. Such a shift in the diagnostic paradigm would have substantial implications for patient management, particularly in the increasingly common scenario era in which asymptomatic meningiomas are incidentally diagnosed on imaging performed for unrelated reasons. In our study, we sought to develop and test methodologies for the pre-operative diagnostic assessment of meningioma grade using two categories of radiographic data (semantic and radiomic) derived from T1-weighted contrast-enhanced MRI.

We observed strong associations between specific radiographic features and meningioma histologic grade. In particular, heterogeneous tumors with necrosis and/or hemorrhage, and irregularly shaped (non-spherical) tumors were more likely to be higher grade on univariate analysis. Two radiomic features, HILAE and LILAE, were sensitive to high and low intensity large areas, respectively, which are commonly indicative of hemorrhage and necrosis on MR images. Interestingly, both semantic and radiomic features were significantly associated with these traits and their presence indicated an increased likelihood of a high grade tumor. Tumor heterogeneity was also significantly associated with more aggressive meningioma grade in both semantic and radiomic feature analyses.

Irregularities in the shape of meningiomas such as "mushrooming" has been previously associated with high grade tumor in multiple studies31-34. Meningioma heterogeneity, on the other hand, is a more complex tumor trait that may be accounted for by a variety of underlying causes, including intratumoral necrosis, cystic degeneration, heterogeneous tumor cell expansion, variability in cell density, and hemorrhage35,36. Tumor radiographic heterogeneity has been extensively studied in glioblastoma, lung cancer, renal cell cancer, and other systemic malignancies and is felt to contribute significantly to treatment resistance and disease relapse37. Awareness of tumor heterogeneity may 
play an important role in assessing treatment response in meningiomas as well, given recent and impending clinical trials assessing novel targeted and immune therapies for aggressive meningiomas38-40.

We further confirmed the ability of radiographic features to classify meningioma grade on an independent validation dataset. Moreover, even though semantic and radiomic features capture some common traits in the tumor (e.g. heterogeneity), the information contained in these "common" features were complementary. Merging both feature sets significantly improved classification performance, indicating an additive effect between qualitative and quantitative imaging analyses. Clinical data, added for comparison, did not classify patients well nor did it add power to the radiographic model in the validation, although this could be limited by selection bias in the variables analyzed.

Associations between tumor characteristics and pre-operative images have been previously investigated41. However, no sets of phenotypic features have been consistently demonstrated to significantly associate with meningioma grade across studies. Differences between meningioma and low grade glioma was investigated using imaging features from T1-weighed and DWI42, however, the study presented several limitations including a small sample size $(n=15)$. Some studies investigating imaging features suggest that benign tumors display higher ADC while malignant tumors have lower ADC values43-45, while others fail to corroborate a similar relationship46-48. These conflicting results may be due to technical factors, such as the region of interest (ROI) defined and feature standardization48,49.

Likewise, our study faces several limitations. Variations in image acquisition and quality can influence quantitative analyses. We attempted to standardize the uniformity of scans by resampling all images with a common voxel spacing to ensure dimension homogeneity and by filtering voxel intensities to reduce outlier values. We used a temporal split to obtain an independent validation dataset, with comparable demographics between the cohorts, in attempt to internally validate our results. External validation from multiple institutions would strengthen these observations in the future. Our clinical model was predicated on common non-radiographic variables that may influence tumor behavior, but may reflect selection bias and data availability in this singleinstitution cohort.

In conclusion, we found a radiographic signature for meningioma grade using standard pre-operative contrast-enhanced MR images. We demonstrated that there is a strong link between the radiographic phenotype of a tumor and its pathology, which may provide a useful tool for precision medicine. Early and accurate prediction of meningioma grade may influence the decision to observe a tumor or to pursue surgery and earlier consideration of adjuvant therapies. Our study highlights the potential clinical impact of integrative imaging analysis in guiding meningioma management. 


\section{REFERENCES}

1. Louis DN, Ohgaki H, Wiestler OD, Cavenee WK, Burger PC, Jouvet A, Scheithauer BW, Kleihues P. The 2007 WHO Classification of Tumours of the Central Nervous System. Acta Neuropathologica. 2007;114(2):97-109.

2. Bondy M, Ligon BL. Epidemiology and etiology of intracranial meningiomas: a review. Journal of NeuroOncology. 1996;29(3):197-205.

3. Lambin P, Rios-Velazquez E, Leijenaar R, Carvalho S, van Stiphout RGPM, Granton P, Zegers CML, Gillies R, Boellard R, Dekker A, Aerts HJWL. Radiomics: Extracting more information from medical images using advanced feature analysis. European Journal of Cancer. 2012;48(4):441-446.

4. Gillies RJ, Kinahan PE, Hricak H. Radiomics: Images Are More than Pictures, They Are Data. Radiology. 2015:151169.

5. Aerts HJWL. The Potential of Radiomic-Based Phenotyping in Precision Medicine: A Review. JAMA Oncology. 2016.

6. Balagurunathan Y, Gu Y, Wang H, Kumar V, Grove O, Hawkins S, Kim J, Goldgof DB, Hall LO, Gatenby RA, Gillies RJ. Reproducibility and Prognosis of Quantitative Features Extracted from CT Images. Translational Oncology. 2014;7(1):72-87.

7. Fried DV, Tucker SL, Zhou S, Liao Z, Mawlawi O, Ibbott G, Court LE. Prognostic Value and Reproducibility of Pretreatment CT Texture Features in Stage III Non-Small Cell Lung Cancer. International Journal of Radiation Oncology*Biology*Physics. 2014.

8. Parmar C, Rios Velazquez E, Leijenaar R, Jermoumi M, Carvalho S, Mak RH, Mitra S, Shankar BU, Kikinis R, Haibe-Kains B, Lambin P, Aerts HJWL. Robust Radiomics Feature Quantification Using Semiautomatic Volumetric Segmentation. Woloschak GE, ed. PLoS ONE. 2014;9(7):e102107.

9. Coroller TP, Grossmann P, Hou Y, Rios Velazquez E, Leijenaar RTH, Hermann G, Lambin P, Haibe-Kains B, Mak RH, Aerts HJWL. CT-based radiomic signature predicts distant metastasis in lung adenocarcinoma. Radiotherapy and Oncology. 2015;114(3):345-350.

10. Coroller TP, Agrawal V, Narayan V, Hou Y, Grossmann P, Lee SW, Mak RH, Aerts HJWL. Radiomic phenotype features predict pathological response in non-small cell lung cancer. Radiotherapy and Oncology. 2016.

11. Parmar C, Leijenaar RTH, Grossmann P, Rios Velazquez E, Bussink J, Rietveld D, Rietbergen MM, HaibeKains B, Lambin P, Aerts HJWL. Radiomic feature clusters and Prognostic Signatures specific for Lung and Head \&amp; Neck cancer. Scientific Reports. 2015;5:11044.

12. Leijenaar RT, Carvalho S, Hoebers FJ, Aerts HJ, van Elmpt WJ, Huang SH, Chan B, Waldron JN, O'sullivan $\mathrm{B}$, Lambin P. External validation of a prognostic CT-based radiomic signature in oropharyngeal squamous cell carcinoma. Acta Oncologica. 2015;(ahead-of-print):1-7.

13. Yip SSF, Coroller TP, Sanford NN, Huynh E, Mamon H, Aerts HJWL, Berbeco RI. Use of registration-based contour propagation in texture analysis for esophageal cancer pathologic response prediction. Physics in Medicine and Biology. 2016;61(2):906-922.

14. Yip SSF, Coroller TP, Sanford NN, Mamon H, Aerts HJWL, Berbeco RI. Relationship between the Temporal Changes in Positron-Emission-Tomography-Imaging-Based Textural Features and Pathologic Response and Survival in Esophageal Cancer Patients. Frontiers in Oncology. 2016;6.

15. Huynh E, Coroller TP, Narayan V, Agrawal V, Hou Y, Romano J, Franco I, Mak RH, Aerts HJWL. CT-based radiomic analysis of stereotactic body radiation therapy patients with lung cancer. Radiotherapy and Oncology. 2016.

16. Antunes J, Viswanath S, Rusu M, Valls L, Hoimes C, Avril N, Madabhushi A. Radiomics Analysis on FLTPET/MRI for Characterization of Early Treatment Response in Renal Cell Carcinoma: A Proof-of-Concept Study. Translational Oncology. 2016;9(2):155-162.

17. Hassan I, Kotrotsou A, Bakhtiari AS, Thomas GA, Weinberg JS, Kumar AJ, Sawaya R, Luedi MM, Zinn PO, Colen RR. Radiomic Texture Analysis Mapping Predicts Areas of True Functional MRI Activity. Scientific Reports. 2016;6:25295. 
18. Aerts HJWL, Velazquez ER, Leijenaar RTH, Parmar C, Grossmann P, Cavalho S, Bussink J, Monshouwer R, Haibe-Kains B, Rietveld D, Hoebers F, Rietbergen MM, Leemans CR, Dekker A, Quackenbush J, et al. Decoding tumour phenotype by noninvasive imaging using a quantitative radiomics approach. Nature Communications. 2014;5.

19. Wang H, Schabath MB, Liu Y, Berglund AE, Bloom GC, Kim J, Stringfield O, Eikman EA, Klippenstein DL, Heine JJ, Eschrich SA, Ye Z, Gillies RJ. Semiquantitative Computed Tomography Characteristics for Lung Adenocarcinoma and Their Association With Lung Cancer Survival. Clinical Lung Cancer. 2015.

20. Gimenez F, Xu J, Liu Y, Liu T, Beaulieu C, Rubin D, Napel S. Automatic annotation of radiological observations in liver CT images. AMIA ... Annual Symposium proceedings / AMIA Symposium. AMIA Symposium. 2012;2012:257-263.

21. Yanagawa M, Tanaka Y, Kusumoto M, Watanabe S, Tsuchiya R, Honda O, Sumikawa H, Inoue A, Inoue M, Okumura M, Tomiyama N, Johkoh T. Automated assessment of malignant degree of small peripheral adenocarcinomas using volumetric CT data: Correlation with pathologic prognostic factors. Lung Cancer. 2010;70(3):286-294.

22. Lee H-J, Kim YT, Kang CH, Zhao B, Tan Y, Schwartz LH, Persigehl T, Jeon YK, Chung DH. Epidermal growth factor receptor mutation in lung adenocarcinomas: relationship with $\mathrm{CT}$ characteristics and histologic subtypes. Radiology. 2013;268(1):254-264.

23. Backer-Grøndahl T, Moen BH, Torp SH. The histopathological spectrum of human meningiomas. Int J Clin Exp Pathol. 2012;5(3):231-242.

24. Pieper S, Halle M, Kikinis R. 3D Slicer. In: IEEE International Symposium on Biomedical Imaging: Nano to Macro, 2004.; 2004:632-635 Vol. 1.

25. Anon. $R$ Core Team (2013). R: A language and environment for statistical computing. $R$ Foundation for Statistical Computing, Vienna, Austria. ISBN 3-900051-07-0, URL http://www.R-project.org/.

26. Schröder MS, Culhane AC, Quackenbush J, Haibe-Kains B. survcomp: an R/Bioconductor package for performance assessment and comparison of survival models. Bioinformatics. 2011;27(22):3206-3208.

27. Benjamini Y, Hochberg Y. Controlling the False Discovery Rate: A Practical and Powerful Approach to Multiple Testing. Journal of the Royal Statistical Society. Series B (Methodological). 1995;57(1):289-300.

28. Liaw A, Wiener M. Classification and Regression by randomForest. R News. 2002;2(3):18-22.

29. Kuhn M. Building predictive models in $\mathrm{R}$ using the caret package. Journal of Statistical Software. 2008;28(5):1-26.

30. Abedalthagafi MS, Ramkissoon SH, Ligon KL, Ligon AH, Folkerth RD, Santagata S. Clinical Molecular Staging in Meningiomas: Prospective Experience of a Single Institution Using Routine FFPE Samples. In: LABORATORY INVESTIGATION.VOI 94. NATURE PUBLISHING GROUP 75 VARICK ST, 9TH FLR, NEW YORK, NY 10013-1917 USA; 2014:433A-433A.

31. Rohringer M, Sutherland GR, Louw DF, Sima AA. Incidence and clinicopathological features of meningioma. Journal of Neurosurgery. 1989;71(5 Pt 1):665-672.

32. Liu Y, Chotai S, Chen M, Jin S, Qi S, Pan J. Preoperative Radiologic Classification of Convexity Meningioma to Predict the Survival and Aggressive Meningioma Behavior. Sherman JH, ed. PLOS ONE. 2015;10(3):e0118908.

33. Servo A, Porras M, Jääskeläinen J, Paetau A, Haltia M. Computed tomography and angiography do not reliably discriminate malignant meningiomas from benign ones. Neuroradiology. 32(2):94-97.

34. Chernov MF, Kasuya H, Nakaya K, Kato K, Ono Y, Yoshida S, Muragaki Y, Suzuki T, Iseki H, Kubo O, Hori T, Okada Y, Takakura K. ${ }^{1} \mathrm{H}-\mathrm{MRS}$ of intracranial meningiomas: what it can add to known clinical and MRI predictors of the histopathological and biological characteristics of the tumor? Clinical Neurology and Neurosurgery. 2011;113(3):202-212.

35. Buetow MP, Buetow PC, Smirniotopoulos JG. Typical, atypical, and misleading features in meningioma. RadioGraphics. 1991;11(6):1087-1106.

36. Nakasu S, Nakasu Y, Nakajima M, Matsuda M, Handa J. Preoperative identification of meningiomas that are highly likely to recur. Journal of Neurosurgery. 1999;90(3):455-462.

37. Gerlinger M, Rowan AJ, Horswell S, Larkin J, Endesfelder D, Gronroos E, Martinez P, Matthews N, Stewart A, Tarpey P, Varela I, Phillimore B, Begum S, McDonald NQ, Butler A, et al. Intratumor heterogeneity and 
branched evolution revealed by multiregion sequencing. The New England Journal of Medicine. 2012;366(10):883-892.

38. Bi WL, Zhang M, Wu WW, Mei Y, Dunn IF. Meningioma Genomics: Diagnostic, Prognostic, and Therapeutic Applications. Frontiers in Surgery. 2016;3:40.

39. Bi WL, Wu WW, Santagata S, Reardon DA, Dunn IF. Checkpoint inhibition in meningiomas. Immunotherapy. 2016;8(6):721-731.

40. Bi WL, Abedalthagafi M, Horowitz P, Agarwalla PK, Mei Y, Aizer AA, Brewster R, Dunn GP, Al-Mefty O, Alexander BM, Santagata S, Beroukhim R, Dunn IF. Genomic landscape of intracranial meningiomas. Journal of Neurosurgery. 2016:1-11.

41. Sachdeva J, Kumar V, Gupta I, Khandelwal N, Ahuja C kamal. A dual neural network ensemble approach for multiclass brain tumor classification: BRAIN TUMOR CLASSIFICATION USING DUAL NETWORK ENSEMBLE APPROACH. International Journal for Numerical Methods in Biomedical Engineering. 2012;28(11):1107-1120.

42. Piper RJ, Mikhael S, Wardlaw JM, Laidlaw DH, Whittle IR, Bastin ME. Imaging signatures of meningioma and low-grade glioma: a diffusion tensor, magnetization transfer and quantitative longitudinal relaxation time MRI study. Magnetic Resonance Imaging. 2016;34(4):596-602.

43. Surov A, Gottschling S, Mawrin C, Prell J, Spielmann RP, Wienke A, Fiedler E. Diffusion-Weighted Imaging in Meningioma: Prediction of Tumor Grade and Association with Histopathological Parameters. Trans/ational Oncology. 2015;8(6):517-523.

44. Tang Y, Dundamadappa SK, Thangasamy S, Flood T, Moser R, Smith T, Cauley K, Takhtani D. Correlation of Apparent Diffusion Coefficient With Ki-67 Proliferation Index in Grading Meningioma. American Journal of Roentgenology. 2014;202(6):1303-1308.

45. Hakyemez B, Yıldırım N, Gokalp G, Erdogan C, Parlak M. The contribution of diffusion-weighted MR imaging to distinguishing typical from atypical meningiomas. Neuroradiology. 2006;48(8):513-520.

46. Santelli L, Ramondo G, Della Puppa A, Ermani M, Scienza R, d’Avella D, Manara R. Diffusion-weighted imaging does not predict histological grading in meningiomas. Acta Neurochirurgica. 2010;152(8):13151319.

47. Pavlisa G, Rados M, Pazanin L, Padovan RS, Ozretic D, Pavlisa G. Characteristics of typical and atypical meningiomas on ADC maps with respect to schwannomas. Clinical Imaging. 2008;32(1):22-27.

48. Sanverdi SE, Ozgen B, Oguz KK, Mut M, Dolgun A, Soylemezoglu F, Cila A. Is diffusion-weighted imaging useful in grading and differentiating histopathological subtypes of meningiomas? European Journal of Radiology. 2012;81(9):2389-2395.

49. Surov A, Ginat DT, Sanverdi E, Lim CCT, Hakyemez B, Yogi A, Cabada T, Wienke A. Use of Diffusion Weighted Imaging in Differentiating Between Maligant and Benign Meningiomas. A Multicenter Analysis. World Neurosurgery. 2016;88:598-602. 



\section{Chapter}

\section{Somatic mutations drive distinct imaging phenotypes in lung cancer}




\section{ABSTRACT}

Tumors are characterized by somatic mutations that drive biological processes which are ultimately reflected in the tumor phenotype. Quantitative radiomics can automatically quantify this radiographic phenotype using artificial intelligence (AI) methods by applying non-invasively characterizes tumor phenotypes by applying a large panel of engineered automatic image characterization algorithms to extract quantitative features from medical images. However, precise genotype-phenotype interactions through which somatic mutations influence radiographic phenotypes remain largely unknown. Here, we present an integrated analysis of independent datasets of 763 lung adenocarcinoma patients with somatic mutation testing and quantitative computed tomography (CT) image analytics that demonstrates somatic mutations are strongly associated with imaging phenotypes. We developed radiomic signatures capable of distinguishing between tumor genotypes in a discovery cohort $(n=351)$ and verified them in an independent validation cohort $(n=$ 352); all radiomic signatures significantly outperformed conventional radiographic predictors (tumor volume and maximum diameter) simple radiographic volumetric predictors. We found a radiomic signature related to radiographic heterogeneity that could strongly discriminate between EGFR+ and EGFR- cases ( $A U C=0.69$ ). Combining this signature with a clinical model of EGFR status ( $A U C=0.70$ ) significantly improved the prediction accuracy $(A \cup C=0.75)$. The highest performing signature was capable of distinguishing between EGFR+ and KRAS+ tumors (AUC=0.80) and when combined with a clinical model ( $A \cup C=0.81$ ), substantially improved its performance ( $A \cup C=0.86)$. A KRAS+/KRAS- radiomic signature also showed significant, albeit lower, performance ( $A \cup C=0.63$ ) and did not improve the accuracy of a clinical predictor of KRAS status. These results suggest that somatic mutations drive distinct radiographic phenotypes that can be predicted using radiomics. Such radiomic-based tests can be applied non-invasively, repeatedly, and at low cost, providing an unprecedented opportunity for precision medicine applications.

\section{SIGNIFICANCE STATEMENT}

We present the first radiomics study to investigate somatic-genotype and imagingphenotype associations in lung cancer in large discovery and validation cohorts. We discovered strong associations between tumor mutations and imaging phenotypes and developed non-invasive imaging-based signatures that are predictive of mutational status in lung cancer patients. These biomarkers outperform standard radiographic volumetric predictors, and generally showed improved performance when combined with clinical predictors, demonstrating complementary value. Such imaging biomarkers have great clinical potential as they can be applied non-invasively and at low additional costs. Furthermore, imaging studies are performed regularly during the course of cancer therapy and could potentially be used to monitor changes in tumor mutational status. 


\section{INTRODUCTION}

Somatic mutations, alterations in the DNA sequence that can occur during an organism's lifetime, are potential biologic drivers of cancers that, in turn, can accelerate the accumulation of further somatic mutations. It is well known that the location of somatic mutations, for example within specific genes, can influence biological processes involved in the development and progression of tumors, ultimately influencing its phenotype.

With the introduction of genomic profiling in clinical practice, cancer treatment decisions are increasingly based not only on the patient's clinical characteristics and tumor morphology, but also on individual mutational profiles(1,2). For example, the use of erlotinib and gefitinib, drugs that target specific mutations within the epidermal growth factor receptor (EGFR) gene, have resulted in improved outcomes in a subset of lung cancer patients in which those mutations occur(3-5). Although mutational sequencing of biopsies can be informative and has become standard of care in some situations, they typically quantify only a small part of a possibly heterogeneous tumor, and they are often only performed once, i.e. prior to initiation of treatment. Further, there are instances in which such screening can be impractical. Repeated tumor sampling, difficult-to-access tissue samples, failure to determine a mutational status due to poor DNA quality, the relative high costs, and long turnaround time can limit the applicability of molecular assays to monitor the cancer progression and its response to treatment (6-8).

Medical imaging is in routine use in oncology for tumor detection, definition of location and extent of disease, treatment planning, and longitudinal response monitoring. Tumor images exhibit strong phenotypic differences between patients (Figure 1a) that can be used to assess tumor phenotype (including effects of the genotype) and its local microenvironment, and these data can be used in determining potential treatments(9). While imaging cannot replace biopsies, imaging studies can provide additional information that biopsies fail to deliver, such as radiographic intra-tumor heterogeneity, and can do so throughout the course of treatment(10), providing longitudinal information on disease state, evolution, and response to therapy.

The quantification of phenotypic characteristics on medical imaging has classically been performed by (semi)-qualitative assessment of radiologists, characterizing so-called "semantic" features(10). Although some studies have shown association of these measures with clinical outcomes, their use has been limited, primarily because they require substantially more work and have shown strong inter- and intra-reader variation. Using advanced image analysis algorithms that originate from Artificial Intelligence

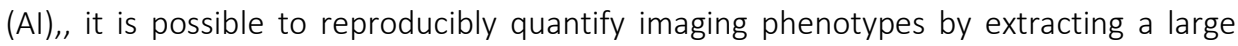
number of image features. $S$ some of these features which capture characteristics that are understandable by human observers and often related to semantic features; others 
capture agnostic characteristics that are generally higher-order and filtered metrics. This automated process of Alimage-based phenotyping is referred to as "radiomics" and can provide a far more detailed characterization of the phenotype than would be possible by eye (Figure 1)(9-13).

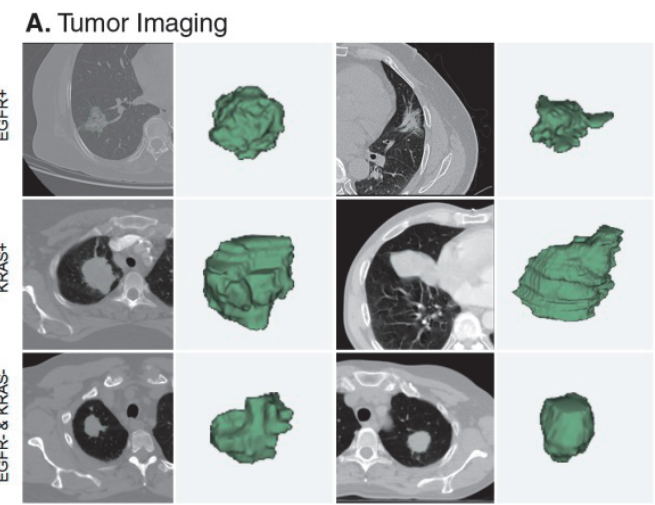

B. Radiomic phenotyping

C. Signature Development
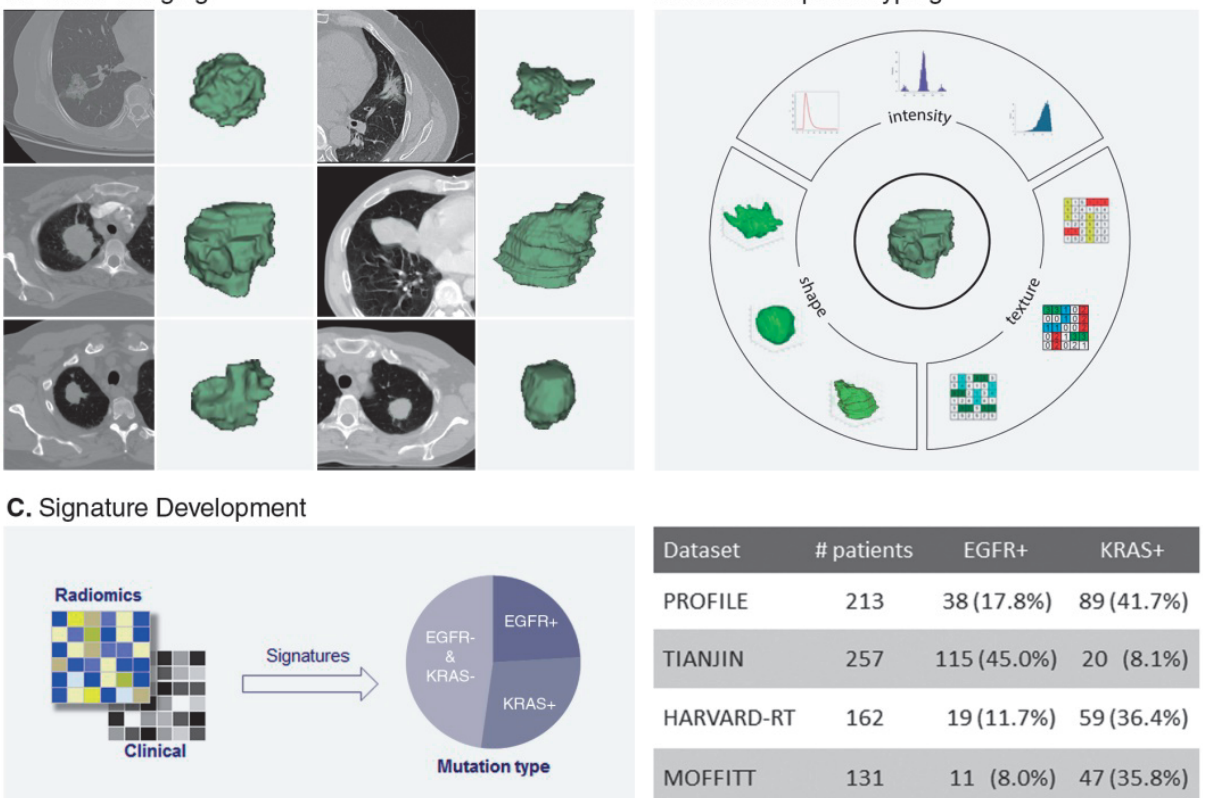

\begin{tabular}{|lccc|}
\hline Dataset & \# patients & EGFR+ & KRAS+ \\
\hline PROFILE & 213 & $38(17.8 \%)$ & $89(41.7 \%)$ \\
\hline TIANJIN & 257 & $115(45.0 \%)$ & $20(8.1 \%)$ \\
\hline HARVARD-RT & 162 & $19(11.7 \%)$ & $59(36.4 \%)$ \\
\hline MOFFITT & 131 & $11(8.0 \%)$ & $47(35.8 \%)$ \\
\hline
\end{tabular}

Figure 1. Analysis workflow. A) Examples of lung adenocarcinomas tumors imaged with computed tomography (CT) imaging (left) and segmented in 3D (right). B) Quantification of the tumor phenotype using radiomics feature algorithms. C) Radiomic and clinical data was used to develop signatures for EGFR and KRAS mutation status from four independent datasets, to investigate associations between the radiomic features and somatic mutations in lung adenocarcinomas. Details on the patient and tumor characteristics of these cohorts are shown in Supplemental Table 1 and Supplemental Data 1.

Radiomic biomarkers have been shown to be associated with several clinical endpoints, including survival $(11,14-16)$, nodule malignancy $(17,18)$, pathological response $(19,20)$, recurrence and distant metastasis (21-23), as well as tumor gene expression patterns $(11,14,21)$. A natural extension of this observation is that tumor phenotype should be linked to the tumor genotype. Given that somatic mutations affect the ability of cells to grow in otherwise non-permissive conditions, we decided to test whether these conditions can be quantified by radiomics and if they reflect the underlying mutational landscape - and whether one could use radiomic phenotype to predict tumor genotype. Although, associations between diagnostic imaging features and mutational data have been explored (24-34), most studies suffer from small cohort sizes, do not include external validation, or have relied on observer-dependent semi-quantitative features that make 
replication difficult. We hypothesized that automated quantitative radiomic feature extraction, applied to a large, heterogeneous cohort, and rigorously validated, could establish the genotype-imaging phenotype linkage.

In this study, we used a cohort of 763 lung adenocarcinoma patients assembled from four institutions to investigate tumor radiomic features extracted from computed tomography (CT) images and tested these features against the most frequently occurring genetic alterations in the disease, i.e. EGFR and KRAS mutations. We used a discovery cohort of 353 patients and identified multivariate radiomic signatures specific for EGFR and KRAS mutations. We validated the predictive power of these signatures to identify EGFR and KRAS mutations in an independent cohort of 352 patients. Finally, we combined these signatures with clinical parameters to create integrated predictors that, in general, exhibited improved performance. Our results argue for integration of robust, reproducible radiomic signatures into clinical practice as they can provide additional information that can be used to assess mutational status and which may allow to predict the emergence of new mutations during the course of therapy.

\section{METHODS AND MATERIALS}

\section{Datasets}

In this study we used the following four independent lung cancer dataset:

- PROFILE: A total of 213 patients with confirmed lung cancer, stages I-IV, were prospectively included, between June 2011 and June 2013, for mass spectrometry genotyping of 471 known mutations in 41 oncogenes and tumor suppressors (PROFILE OncoMap) as described previously $(45,47)$. Tumor genomic profiling and clinical data of all patients were retrieved from the Clinical and Operational Research Information System of the Dana-Farber Cancer Institute and Brigham and Women's Hospital. All patients with a biopsy performed in the primary tumor were selected. We excluded cases whose histological type was not of lung origin.

- TIANJIN: A total of 257 surgical patients with lung adenocarcinoma, stages I-IV, with data on EGFR and KRAS mutations were included in our analysis. For all patients, diagnostic CT imaging, tumor delineations and EGFR and KRAS mutation status were available.

- MOFFITT: A cohort of 131 lung cancer patients treated at the Moffitt cancer center were included in our analysis. Clinical stages ranged from stage I to stage IV. For these patients, mutation status was determined using mass spectrometry in the KRAS, EGFR, TP53, STK11 genes.

- HARVARD-RT: A cohort of 162 patients with lung adenocarcinoma, stages I-IIIb, treated with radiation oncology at the Dana-Farber Cancer Institute and Brigham 
and Women's Hospital. Data on EGFR and KRAS mutations were routinely clinically collected and extracted from the electronic medical records.

The Institutional Review Boards of each of the participating centres approved the studies: Profile and Harvard-RT (Dana-Farber/Harvard Cancer Center IRB, Boston, MA), Tianjin (Tianjin Medical University IRB, Tianjin, China) and Moffitt (IRB Moffitt Cancer Center, Tampa, FL). Details on the patient's and tumor characteristics as well as data available are provided on Supplementary Table 1 and Supplementary Figure 1. Analysis of this study was performed under an institutional review board within the Consented Research Data Repository of the Dana-Farber/Harvard Cancer Center. In Supplementary Information II a detailed description about the datasets are available.

\section{Radiomic quantification}

The tumor imaging phenotype was described using a set of quantitative radiomic features extracted from the segmented tumor regions on the CT scans. Briefly, CT images and tumor contours were imported into 3D-Slicer in NRRD format. Due to the differences in pixel spacing and slice thickness, the images and tumor contours were subsequently normalized to isometric voxels $(3 \mathrm{~mm}$ ) using a cubic interpolation. Next, feature extraction was performed using an in-house developed Radiomics plug-in for 3D-Slicer. All features have been described in detail previously $(11,21)$. Features were grouped as follows:

I. Tumor intensity features. These include first-order statistics, calculated from the histogram of all tumor voxel intensity values.

II. Textural features. These quantify intra-tumor heterogeneity and are calculated in all 3-dimensional directions within the tumor volume, thereby taking the spatial location of each voxel compared to the surrounding voxels into account. The size-zone matrix was used to quantify regional heterogeneity. This matrix allows characterization of arrangements of voxels within the tumors, therefore describing tumor regional heterogeneity.

III. Shape features. Metrics of the three-dimensional shape and size of the tumor.

IV. Wavelet features: Features in groups I and II are extracted after applying a series of wavelet transforms to the CT images. The wavelet transform decomposes the original image into low - and high - frequencies, thereby focusing the features on different frequency ranges within the tumor volume.

V. Laplacian of Gaussian features: These are textural features extracted after in-plane filtration using a Laplacian of Gaussian spatial band-pass filter. This filter highlights textural and anatomical patterns of different width depending of the spatial scale of the filter. By modifying the filter width, fine, medium and coarse textures can be highlighted and textural features are subsequently calculated. 


\section{Data analysis}

First, we investigated the associations between the imaging phenotype and the most common somatic mutations in the integrated dataset (Figure 1). We used an unsupervised two-step feature selection methodology. First, we used the RIDER NSCLC testretest dataset $(n=31)$ to assess stability of the radiomic features $(48)$. For each patient, we extracted radiomic features from the test and re-test scans. The intra-class correlation coefficient (ICC) was used to determine the stability of the features. Features with an ICC lower than 0.8 were excluded from the analysis. In a second step, we performed a principal component (PCA) based analysis(49) to identify the highest correlated features (Pearson's $r>0.90$ ) to the principal components that describe at least $90 \%$ of the variance in the radiomic data. This resulted in a selection of 26 variance retaining features. Tumor volume and axial diameter were added for comparison. We compared the radiomic features distributions between mutated and non-mutated cases for each gene using a two-sided Wilcoxon test. To correct for multiple comparisons, we adjusted Pvalues by the false-discovery-rate $(F D R=5 \%)$ procedure according to Benjamini and Hochberg(50).

The ability to predict the mutational status of the radiomic features was assessed by the area under the curve (AUC) of the receiver operator characteristic (ROC) as implemented in the survcomp R package (Version 1.12.0)(51). Significance of AUCs was determined using the "noether" method implemented in the R survcomp package.

For the multivariate analysis, we used a temporal split (median scan acquisition date) to divide each of the four cohorts into training and validation sets. All training cohorts were combined into an integrated discovery cohort to identify radiomic signatures for EGFR and KRAS mutations while the validation cohorts were combined into an integrated validation dataset. In order to statistically compare radiomics and clinical multivariate models, we excluded all the samples with any missing clinical (stage, gender, smoking status, age, or race) or mutation (EGFR or KRAS status) information from each of the four cohorts before the temporal split. This exclusion resulted in total 257, 186, 142 and 120 samples in Tianjin, Profile, Lung-RT and Spore-Moffitt cohorts respectively, and hence, 353 patients were used for discovery and 352 patients for independent validation. In order to compare the two positive mutations (EGFR+ and KRAS+), we also excluded the wild type cases (EGFR- and KRAS-) from each cohort before the temporal split. This further reduction resulted in total 136, 114, 78 and 53 samples in Tianjin, Profile, Lung-RT and Spore-Moffitt cohorts respectively, and hence, 190 patients were used for discovery and 191 patients for independent validation. Radiomic signatures to predict mutation status were built in the integrated discovery cohort, by minimum redundancy maximum relevance (MRMR) feature selection. MRMR has been shown previously to be a stable feature selection algorithm for radiomics(52). The MRMR 
algorithm(53) was applied on all radiomic features with respect to a given mutational status, i.e. EGFR, to select a non-redundant and highly informative set of features. Using the top 20 MRMR ranked features, controlling for differences in event ratios, we trained a random forest classifier on the discovery cohort. RF classifiers have also shown stability and high accuracy on radiomics analyses $(15,52,54,55)$. RF models were built for radiomic features and mutation data on the discovery cohort and their performance was evaluated on the validation cohort, therefore none of the models were overfitted because models were trained only on the discovery cohort. The prediction performance was assessed using area under receiver operator characteristics curve (AUC). We also built random forest based clinical multivariate models using five clinical variables, i.e. tumor stage, gender, smoking status, age and race. Distributions of these clinical variables across the four cohorts can be obtained from the patient characteristics table (Supplementary Table 1). In order to assess the additive effect in prediction, combined models with 20 MRMR ranked radiomic features and 5 clinical variables were built using random forest. Prediction performance (AUC) of these different models were statistically compared using one sided t-test as implemented in the R package survcomp. To compute additional prediction measures (e.g. Sensitivity, Specificity, Accuracy, NPV and PPV), we used the event (mutation) ratios of the discovery cohort as a probability threshold and obtained a corresponding cutoff point on the ROC curves. These prediction measures were computed using the R package pROC. MRMR feature selection was implemented using the MATLAB toolbox FEAST(53) and Matlab (Version R2012b, The Mathworks, Natick, MA). All other statistical analyses were performed using R (Version 3.0.2).

\section{RESULTS}

\section{Genotype-phenotype associations}

To investigate genotype-phenotype associations, we compared computed tomography (CT) radiomic features with somatic mutation status in lung adenocarcinoma patients (Figure 1). To incorporate the diversity of genotypic and phenotypic variations between the individual cohorts (see Supplementary Figure 1), we performed an integrated analysis combining four cohorts, totaling 763 patients (Supplementary Table 1). TWe identified twenty-six robust and non-redundant radiomic features were included in our analysis. These features were selected based on test-retest performance, to assess stability of the radiomic features, and based on an unsupervised feature selection methodology to select uncorrelated features (see Methods). Selected features, includedding intensity histogram metrics, shape, and texture features - with or without wavelet or Laplacianof-Gaussian filters. These features were selected in an unsupervised fashion based on 
test-retest performance and on inter-feature correlation, and independent of mutation status or any other outcome to minimize overfitting (see Methods) Supplementary Data I). Selected features included intensity histogram metrics, shape, and texture features with or without wavelet or Laplacian-of-Gaussian filters (see Supplementary Data I). These features are capable to quantify a panel of phenotypic characteristics, such as intra-tumor homogeneity and heterogeneity, tumor density, and spherical disproportion, describing tumor roundness.

We then investigated the association of selected features with the most frequent somatic mutations in lung adenocarcinoma, KRAS (28.2\%, 215 of 763) and EGFR (24\%, 183 of 763). Using a non-parametric, two-sided Wilcoxon-test on the integrated cohort and correcting for multiple testing ( $5 \%$ FDR), we separately compared EGFR mutated and KRAS mutated tumors to cases without EGFR or KRAS mutations, respectively.

We found sixteen radiomic features to be significantly associated with EGFR mutations and ten features associated with KRAS mutations (Figure 2). Significant features were reported as overrepresented (+) or underrepresented (-) indicating the relative feature representation.

For EGFR mutated tumors, we found the Homogeneity and Inverse Variance radiomic features to be underrepresented whereas Sum Entropy and Short Run Emphasis were overrepresented. Homogeneity is sensitive to the number of unique discrete values in the images such that the fewer unique values that are accessed, the more homogeneous the image. Inverse variance assesses variations in intensity of voxels close to each other and therefore quantifies another aspect of homogeneity. Sum Entropy is the entropy of the co-occurrence matrix and therefore quantifies complexity. Short Run Emphasis is a run length feature that indicates successive voxels have similar intensity values. Together, the representation of these features indicate that EGFR positive tumors are more likely to be heterogeneous.

In contrast, Sum Entropy was underrepresented for KRAS mutated tumors, indicating that KRAS mutants are more homogeneous. Total Energy is associated with a LLL wavelet filter, which is a low pass filter that enhances the general information of the image while reducing its noise. Total Energy was underrepresented in EGFR mutated tumors and overrepresented in KRAS mutated tumors so that EGFR mutated tumors had a quantitatively lower Total Energy metric, compared to KRAS mutated tumors.

We then compared radiographic features between EGFR and KRAS mutant tumors. We found fourteen significant features all of which were among the sixteen that distinguished EGFR mutant from EGFR non-mutated tumors. This pronounced difference between EGFR mutated tumors and others is consistent with tumor volumetric analysis. EGFR mutant tumors were smaller than non-mutated tumors $\left(18.15 \pm 81.7 \mathrm{~cm}^{3}\right.$ vs $29.7 \pm 61.1 \mathrm{~cm}^{3}$, FDR $\left.p<0.05\right)$ while KRAS mutant tumors were more similar in size to non-mutated tumors $\left(29.6 \pm 62.7 \mathrm{~cm}^{3}\right.$ vs $\left.25.6 \pm 69.2 \mathrm{~cm}^{3}, \mathrm{p}=0.365\right)$. 


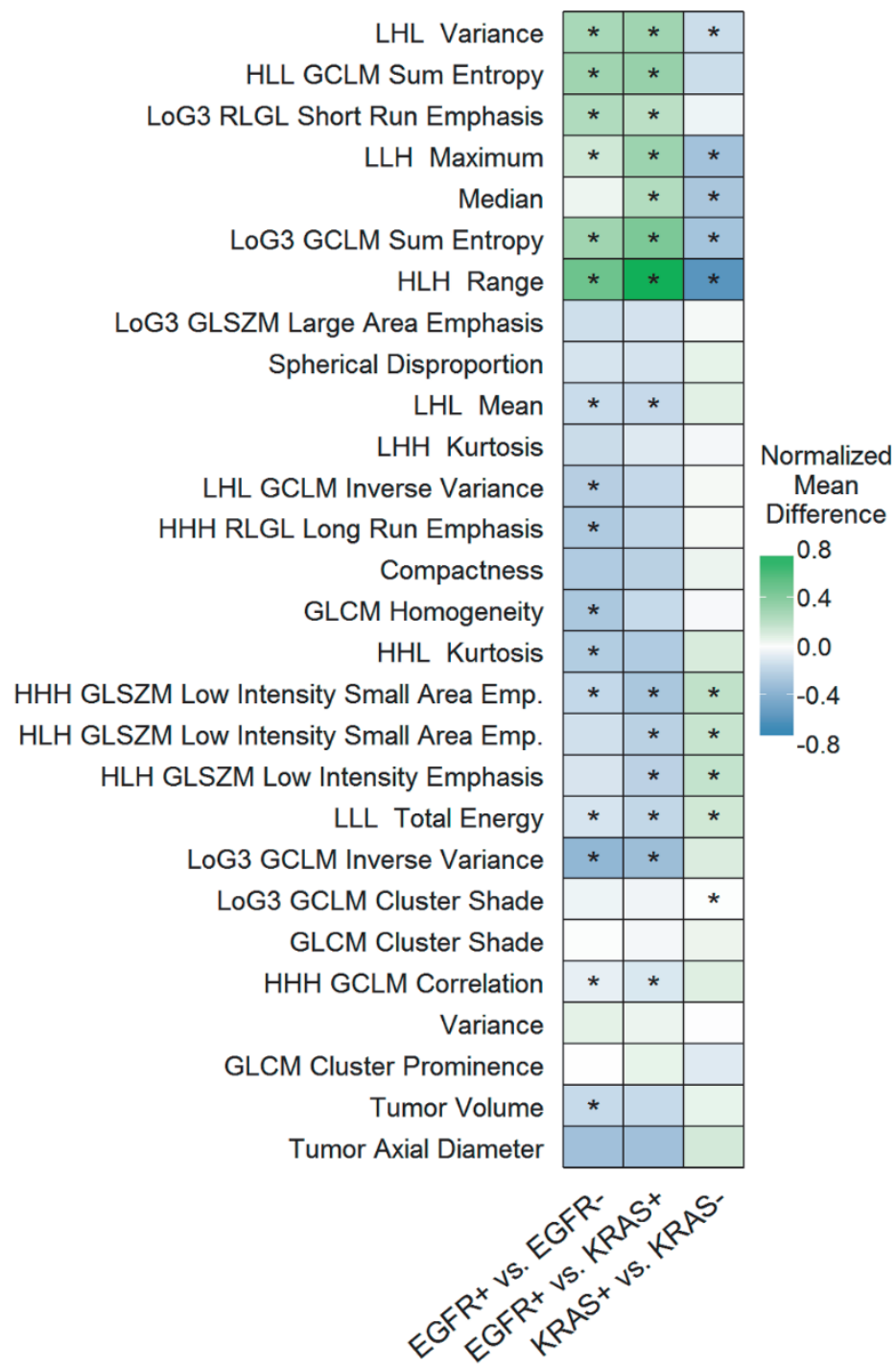

Figure 2. Somatic genotype - imaging phenotype associations by comparing radiomic feature distributions between mutation subtypes. Heatmap shows the normalized mean difference of radiomic feature distributions for twenty-six reproducible and variant imaging features of 763 lung adenocarcinoma patients. Volumetric features were included for comparison. ${ }^{*}$ Indicates $5 \%$ false discovery rate (FDR) corrected $p$-values using a two-sided Wilcoxon test. Note, that many features are significantly different between EGFR+ vs EGFR- and between EGFR+ and KRAS+, although less for KRAS+ vs KRAS-.

\section{Predictive radiomic signatures for EGFR and KRAS mutation status}

To evaluate the value of radiomic data to predict EGFR and KRAS mutation status, we developed and independently validated radiomic signatures, and compared their per- 
formance with clinical models (Figure 1c). To incorporate the diversity of genotypic and phenotypic variations across the datasets into the signature development, we divided each of the four cohorts into an independent training set $(n=353)$ and an independent validation set $(n=352)$ (Supplementary Figure 2). Three radiomic signatures were developed for classifying: I) between EGFR+ and EGFR-, II) between KRAS+ and KRAS-, and III) between EGFR+ and KRAS+. Furthermore, we also developed clinical models incorporating age, gender, smoking status, race, and clinical stage, to classify between these three groups. The performances of the radiomic and clinical signatures were compared to each other, and to conventional radiographic parameters used in clinical settings (axial diameter and volume of the tumor).

Each of the three signatures used twenty radiomic features (see Methods and Supplementary Methods 3). It is noteworthy that a large number of included features (9 out of 20), were common across the three signatures. These included textural features that are sensitive to tumor radiographic heterogeneity, such as gray-level non-uniformity (GLNU) and low intensity small area emphasis (LISAE), both sensitive to complex patterns or high variation, as well as cluster prominence (CP) and inverse difference moment (IDM), which emphasize voxel pattern from close range intensity (e.g. smooth transition between voxel intensity), and are related to radiographic homogeneity.

Figure 3 and Supplementary Tables 2-4 show the performances of radiomic signatures on the validation cohort. Conventional radiographic predictors, i.e. maximum diameter and tumor volume showed significant, albeit low, performance in distinguishing between EGFR+ and EGFR- tumors ( $A \cup C=0.61, p=5.88 \times 10^{-04}$ and AUC=0.60, $p=8.44 \times 10^{-04}$, respectively). Neither diameter nor volume were able to distinguish between KRAS+ and KRAS- (AUC $=0.53, p=0.44$ and $A \cup C=52, p=0.63$, respectively), or to distinguish between $E G F R+$ and $K R A S+(A \cup C=0.58, p=0.07$ and $A \cup C=0.56, p=0.12$, respectively). 

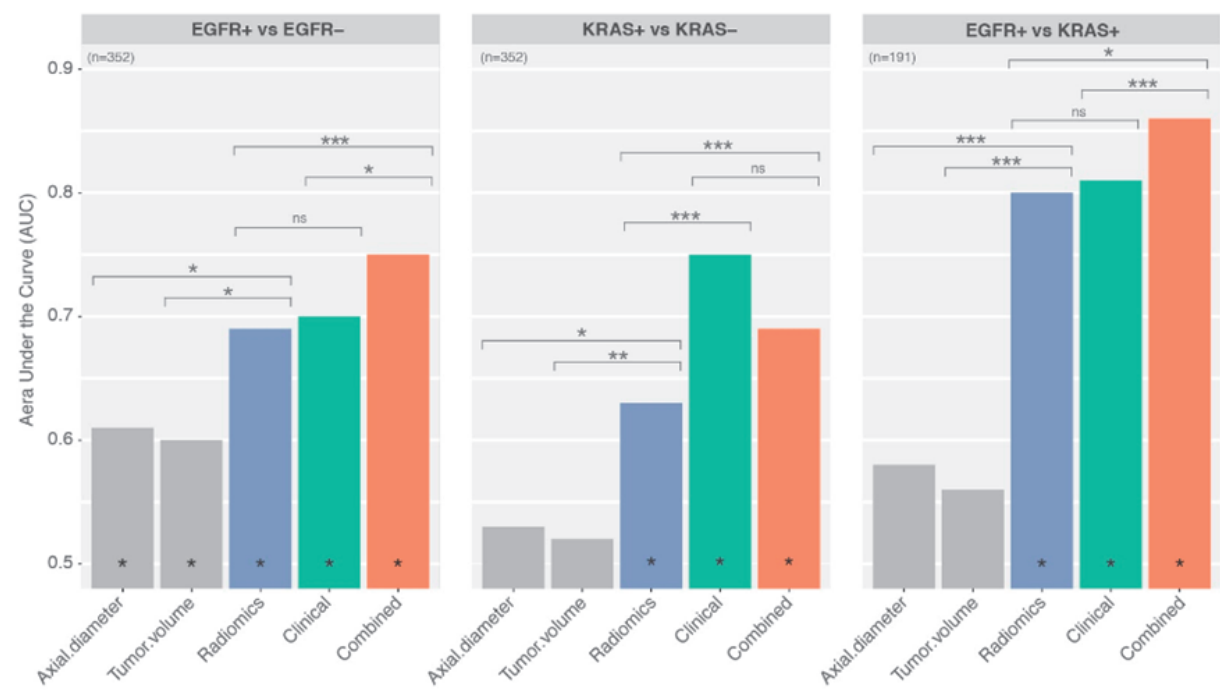

Figure 3. Radiomic signatures to predict somatic mutations. Performance of radiomic signatures on the validation cohort for each mutational status classification on an independent validation dataset ( $\mathrm{n}=353)$. For comparison, conventional radiographic parameters (axial diameter and tumor volume) were included. Clinical models, including age, gender, smoking status, race, and clinical stage, were developed for each classification. * on bottom of bars indicates that the performance of a model is significantly better than random. Furthermore, the segments indicate if a signature is significantly higher than another $\left(^{*}=p<0.05, * *=p<0.01, * * *=\right.$ $\mathrm{p}<0.001, \mathrm{~ns}=$ not significant). Note, that overall the radiomic signatures significantly outperform radiographic volumetric predictors.

The radiomic signature we developed showed a significant ability to discriminate between $E G F R+$ and EGFR- cases ( $A U C=0.69, p=5.32 \times 10^{-10}$, Figure $3 A$ ). This signature significantly outperformed axial diameter $(p<0.03)$ and tumor volume $(p<0.02)$. A clinical model of EGFR status, including age, gender, smoking status, race, and clinical stage, also showed high performance ( $A \cup C=0.70, p=1.71 \times 10^{-09}$ ), and was similar to the performance of the radiomic signature $(p=0.46)$. We tested whether the radiomic and clinical signatures were complementary by creating a combined predictor and identified a combined signature that improved the accuracy $\left(A \cup C=0.75, p=8.93 \times 10^{-18}\right.$ ), significantly better than the radiomic $\left(p=0.05 \times 10^{-02}\right)$ and clinical $(p=0.03)$ signatures alone. The complementary power of the two signatures was supported by a higher sensitivity for the radiomic signature and greater specificity for the clinical signature alone, with the combined signature having both high sensitivity and specificity (Supplementary Table 4,

\section{Supplementary Figure 2).}

A KRAS+/KRAS- radiomic signature also showed significant, albeit lower, performance ( $\left.A \cup C=0.63, p=5.45 \times 10^{-05}\right)$, and outperformed maximum diameter $(p=0.02)$ and tumor volume $(p=0.01)$ (Figure $3 B$ ). A clinical predictor of KRAS, that included age, gender, smoking status, race, and clinical stage, status showed a very high performance ( $A \cup C=0.75, p=5.86 \times 10^{-19}$ ), and was significantly higher than the radiomic model 
$\left(p=8.73 \times 10^{-05}\right)$. Combining both signatures reduced the overall performance ( $A \cup C=0.69$ ). In fact, the accuracy was higher for the clinical signature (0.66) than for either the radiomic (0.56) or combined (0.60) signature (Supplementary Table 4, Supplementary Figure 2).

Finally, we developed a predictive signature to distinguish between EGFR mutated and KRAS mutated tumors(Figure $3 \mathrm{C}$ ). This radiomic signature had the best overall performance $\left(A \cup C=0.80, p=1.20 \times 10^{-20}\right.$ ), significantly higher than either maximum diameter $\left(p=1.65 \times 10^{-05}\right)$ or tumor volume $\left(p=6.17 \times 10^{-06}\right)$. Combining this signature with a clinical signature $\left(A \cup C=0.81, p=1.37 \times 10^{-21}\right.$ ), substantially improved its performance $(A \cup C=0.86$, $\left.p=9.88 \times 10^{-39}\right)$. This improvement was also significantly higher than either the radiomic or clinical model alone $\left(p=0.02 \times 10^{-02}\right.$ and $p=0.02$, respectively). The combined model also had a high specificity (0.87) and accuracy (0.79) (Supplementary Table 4, Supplementary Figure 2).

\section{DISCUSSION}

Cancer is characterized by distinct molecular and environmental events that drive tumor development and progression $(35,36)(35,36)$. Radiomic assessment of the tumor phenotype can be used with non-invasive images that are collected routinely in the clinic throughout the course of care. Our driving hypothesis is that the tumor phenotype, measured quantitatively through radiomics, should reflect the tumor genotype. We investigated the association between computed tomography (CT) radiomic phenotypes and the most common somatic mutations in lung adenocarcinoma, i.e. EGFR and KRAS mutations. We performed an integrated analysis of four large independent cohorts of lung adenocarcinoma patients for whom clinical, imaging, and mutational profiling data were available. We applied a stringent statistical design with independent training and validation cohorts in a large number of patients, to ensure validity of results. We found that EGFR tumor were more likely to be heterogeneous, with a smaller volume, and presenting an overall lower density on CT images. On the other hand, KRAS tumors could not be discriminated based on volumetric information, however they were more likely to be homogeneous.

We developed radiomic signatures predictive of mutational status in a discovery cohort of 353 patients that showed strong predictive performance in a validation cohort of 352 patients. We found radiomic signatures were predictive of EGFR mutations ( $A \cup C=0.69$ ) and were able to reliably distinguish between EGFR+ and KRAS+ (AUC $=0.80)$ tumors. The EGFR+/EGFR- radiomic signature complemented the predictive value of a signature based on clinical factors (AUC $=0.75)$. 
Few studies have investigated associations between the tumor imaging phenotype and the underlying molecular landscape (24-31,33). These studies generally had small sample sizes, used subjective observer-dependent imaging descriptors, and did not perform robust external validation. For example, associations have been reported between textural imaging features and KRAS mutations in combined imaging modalities (CT, ${ }^{18} \mathrm{~F}$ FDG PET and dynamic contrast-enhanced CT) in colorectal cancer(25), and using CT in $\operatorname{NSCLC}(27,37)$, but within small cohorts (<100 patients), and, more importantly, without external validation, which is a critical component of radiomic analyses $(9,10)$. Our analysis of radiomic features extracted from CT images found that they had relatively weak predictive power for KRAS, a result that we validated in an independent validation set, indicating a generally similar imaging phenotype for KRAS-positive and negative tumors. For instance, Wang et al., found a single semantic CT feature, tumor spiculation, to be associated with the presence of KRAS mutations in a single cohort of stage I lung adenocarcinoma patients(37). We found, however, that EGFR-positive and negative tumors exhibit distinct imaging phenotypes, allowing more sensitive and specific classification of tumor EGFR status. In a recent single-cohort association study, Liu et al. found 16 semantic CT annotations to be associated with EGFR status in a cohort of NSCLC patients. EGFR mutations were associated with tumor size, with ground glass opacity, and enhancement heterogeneity. Their study however lacked validation of their multivariate models and did not include multiple testing corrections(38). Similarly, Park et al, in a single-cohort association study of advanced stage lung adenocarcinomas, found that EGFR positive tumors presented more frequently ground-glass opacity, while KRAS positive cases were more likely solid tumors(39).

This is the first study to evaluate associations between somatic mutations and radiomic features in a large cohort of lung adenocarcinoma patients, and to validate its findings in an independent patient cohort. Our findings suggest the need for studies in other cancers to learn more general rules for mapping clinical and imaging features to the tumor's mutational status in single tissues and across tissues, and to understand whether temporal changes in tumor morphology can be associated with changes in tumor mutational status.

Despite strong results, our study has a number of limitations. We used diagnostic CT scans as performed routinely in the clinic with heterogeneous scanning protocols. CT images were acquired using scanners manufactured by different companies, with a range of image reconstruction algorithms, different slice thicknesses, with and without contrast, and using different dosages. We normalized all images to iso-volumetric voxels to reduce the effect of different slice thicknesses. However, despite these factors potentially adding noise to the data, we were able to identify a strong signal predictive of EGFR mutations. It is conceivable that heterogeneity in CT protocols could have obscured more subtle differences in the phenotype of KRAS positive and negative tumors. Further optimization and standardization of imaging data is an important aspect for the 
introduction of imaging based biomarkers. Several groups, such as the Quantitative Imaging Network(40), investigateaddress these issues by implementing feature standardization efforts such as segmentation challenges, radiomic feature definition standardizations, and developingstimulation to open-source, publicly available analyses platformssoftware.

Radiomic features report 3-dimensional scores that are representative of the tumor as a whole, i.e. as an average score for textural features in all directions in the 3-dimensional space. We believe that a detailed analysis of the intra-tumoral tumor heterogeneity, by compartment mappings, particularly if paired with multiple biopsies at distinct geographical locations, such as investigated in renal cell carcinomas $(7,41)$, may allow us to further elucidate intra-tumoral genotype-phenotype relationships. Localized and temporal tumor heterogeneity represents a challenge for repeated tissue sampling for genomic assays(6), thus, imaging signatures may be useful as a surrogate for genomic assays when a biopsy is not possible, or as complementary assay to monitor response to therapy. Similarly, if the radiomic signature predicts a different EGFR status compared to tissue assessment, particularly if an EGFR targeted treatment was to be delivered, this could indicate re-biopsy, to rule out sampling error or misdiagnosis(42). Furthermore, quantitative radiomic analysis can add in the evaluation of treatment response in EGFR mutant lung adenocarcinoma patients, treated with EGFR tyrosine kinase inhibitors, beyond tumor volume assessments and RECIST criteria(43). This, however, still needs to be evaluated in clinical data.

There are fundamental differences between radiology and pathology based tests. Radiology can capture the phenotype at a macroscopic level (millimeter resolution), and not a microscopic level as provided by histopathology and required for a detailed quantification of underlying biological processes. An advantage of imaging is that it easily can sample the complete disease burden sequentially over time. Therefore, imaging-based biomarkers could potentially be applied in clinical situations were biopsy based assays are not possible, and it could also provide complementary information, specially over the course of treatment. It might also be interesting to compare radiomics-based genotype predictions with liquid biopsy-based information since both of these approaches can reflect the overall tumor load as opposed to the partial sampling provided by biopsies (44).

Nevertheless, this study lays important groundwork for establishing radiomics as an important adjunct approach to existing clinical predictors of disease status and therefore treatment protocol. Ongoing prospective data collection projects such as PROFILE at the Dana-Farber/Brigham and Women's Cancer Center are collecting comprehensive mutational data on almost all lung cancer patients receiving treatment(45) and these data are linked to comprehensive clinical records including radiological scans collec- 
tions. Such initiatives are needed to further validate imaging based predictors that can be useful for clinical application.

In summary, we demonstrated an association between the imaging phenotype captured with a radiomic signature and EGFR mutant tumors, in four independent cohorts of lung adenocarcinomas. This association may have clinical impact in selecting patients for targeted therapies. Imaging phenotype associations with other molecular subtypes of NSCLC should to be further investigated in prospective genotype profiling cohorts $(33,46)$.

\section{ACKNOWLEDGEMENTS}

E.R.V., C.P., and H.J.W.L.A conceived the project, analysed the data and wrote the paper. Y.L., T.P.C., G.C., O.S., Z.Y., F.F., collected data and provided analysis or annotations on the datasets. J.Q., provided bioinformatics support. M.M, R.H.M., R.G., provided expert knowledge, collection and availability of the data. All authors edited the manuscript and approved it before submission. 


\section{REFERENCES}

1. Chin L, Andersen JN, Futreal PA. Cancer genomics: from discovery science to personalized medicine. Nat Med. 2011;17:297-303.

2. Garraway LA, Verweij J, Ballman KV. Precision oncology: an overview. J Clin Oncol. jco.ascopubs.org; 2013;31:1803-5.

3. Janne PA, Engelman JA, Johnson BE. Epidermal growth factor receptor mutations in non-small-cell lung cancer: implications for treatment and tumor biology. J Clin Oncol. 2005;23:3227-34.

4. Li T, Kung HJ, Mack PC, Gandara DR. Genotyping and genomic profiling of non-small-cell lung cancer: implications for current and future therapies. J Clin Oncol. 2013;31:1039-49.

5. Chong CR, Janne PA. The quest to overcome resistance to EGFR-targeted therapies in cancer. Nat Med. 2013;19:1389-400.

6. Bedard PL, Hansen AR, Ratain MJ, Siu LL. Tumour heterogeneity in the clinic. Nature. 2013;501:355-64.

7. Swanton C. Intratumor heterogeneity: evolution through space and time. Cancer Res. 2012;72:4875-82.

8. Taniguchi K, Okami J, Kodama K, Higashiyama M, Kato K. Intratumor heterogeneity of epidermal growth factor receptor mutations in lung cancer and its correlation to the response to gefitinib. Cancer Sci. 2008;99:929-35.

9. Gillies RJ, Kinahan PE, Hricak H. Radiomics: Images Are More than Pictures, They Are Data. Radiology. $2015 ; 151169$.

10. Aerts HJWL. The Potential of Radiomic-Based Phenotyping in Precision Medicine: A Review. JAMA Oncol [Internet]. 2016; Available from: http://dx.doi.org/10.1001/jamaoncol.2016.2631

11. Aerts H, Rios Velazquez E, Leijenaar R, Parmar C, Grossmann P, Cavalho S, et al. Decoding the tumor phenotype by non-invasive imaging using a quantitative radiomics approach. Nat Commun. 2014;

12. Lambin P, Rios-Velazquez E, Leijenaar R, Carvalho S, van Stiphout RG, Granton P, et al. Radiomics: extracting more information from medical images using advanced feature analysis. Eur J Cancer. 2012;48:441-6.

13. Yip SSF, Aerts HJWL. Applications and limitations of radiomics. Phys Med Biol. 2016;61:R150-66.

14. Fried DV, Tucker SL, Zhou S, Liao Z, Mawlawi O, Ibbott G, et al. Prognostic value and reproducibility of pretreatment CT texture features in stage III non-small cell lung cancer. Int J Radiat Oncol Biol Phys. 2014;90:834-42.

15. Parmar C, Leijenaar RTH, Grossmann P, Rios Velazquez E, Bussink J, Rietveld D, et al. Radiomic feature clusters and prognostic signatures specific for Lung and Head \& Neck cancer. Sci Rep. 2015;5:11044.

16. Zhou Y, He L, Huang Y, Chen S, Wu P, Ye W, et al. CT-based radiomics signature: a potential biomarker for preoperative prediction of early recurrence in hepatocellular carcinoma. Abdom Radiol (NY) [Internet]. 2017; Available from: http://dx.doi.org/10.1007/s00261-017-1072-0

17. Hawkins S, Wang H, Liu Y, Garcia A, Stringfield O, Krewer H, et al. Predicting Malignant Nodules from Screening CT Scans. J Thorac Oncol. 2016;11:2120-8.

18. Schabath M, Balagurunathan $Y$, Dmitry G, LAwrence H, Samuel H, Stringfield O, et al. Radiomics of lung cancer. J Thorac Oncol. 2016;11:S5-6.

19. Coroller TP, Agrawal V, Huynh E, Narayan V, Lee SW, Mak RH, et al. Radiomic-Based Pathological Response Prediction from Primary Tumors and Lymph Nodes in NSCLC. J Thorac Oncol. 2017;12:467-76.

20. Coroller TP, Agrawal V, Narayan V, Hou Y, Grossmann P, Lee SW, et al. Radiomic phenotype features predict pathological response in non-small cell lung cancer. Radiother Oncol. 2016;119:480-6.

21. Coroller TP, Grossmann P, Hou Y, Rios Velazquez E, Leijenaar RT, Hermann G, et al. CT-based radiomic signature predicts distant metastasis in lung adenocarcinoma. Radiother Oncol. 2015;114:345-50.

22. Huynh E, Coroller TP, Narayan V, Agrawal V, Hou Y, Romano J, et al. CT-based radiomic analysis of stereotactic body radiation therapy patients with lung cancer. Radiother Oncol [Internet]. 2016; Available from: http://dx.doi.org/10.1016/j.radonc.2016.05.024 
23. Huynh E, Coroller TP, Narayan V, Agrawal V, Romano J, Franco I, et al. Associations of Radiomic Data Extracted from Static and Respiratory-Gated CT Scans with Disease Recurrence in Lung Cancer Patients Treated with SBRT. PLoS One. 2017;12:e0169172.

24. Karlo CA, Di Paolo PL, Chaim J, Hakimi AA, Ostrovnaya I, Russo P, et al. Radiogenomics of clear cell renal cell carcinoma: associations between CT imaging features and mutations. Radiology. 2014;270:464-71.

25. Miles KA, Ganeshan B, Rodriguez-Justo M, Goh VJ, Ziauddin Z, Engledow A, et al. Multifunctional imaging signature for V-KI-RAS2 Kirsten rat sarcoma viral oncogene homolog (KRAS) mutations in colorectal cancer. J Nucl Med. 2014;55:386-91.

26. Nair VS, Gevaert O, Davidzon G, Napel S, Graves EE, Hoang CD, et al. Prognostic PET 18F-FDG uptake imaging features are associated with major oncogenomic alterations in patients with resected non-small cell lung cancer. Cancer Res. 2012;72:3725-34.

27. Weiss GJ, Ganeshan B, Miles KA, Campbell DH, Cheung PY, Frank S, et al. Noninvasive image texture analysis differentiates K-ras mutation from pan-wildtype NSCLC and is prognostic. PLoS One. 2014;9:e100244.

28. Yamamoto S, Korn RL, Oklu R, Migdal C, Gotway MB, Weiss GJ, et al. ALK Molecular Phenotype in NonSmall Cell Lung Cancer: CT Radiogenomic Characterization. Radiology. 2014;272:568-76.

29. Zhou JY, Zheng J, Yu ZF, Xiao WB, Zhao J, Sun K, et al. Comparative analysis of clinicoradiologic characteristics of lung adenocarcinomas with ALK rearrangements or EGFR mutations. Eur Radiol. 2015;25:125766.

30. Rizzo S, Petrella F, Buscarino V, De Maria F, Raimondi S, Barberis M, et al. CT Radiogenomic Characterization of EGFR, K-RAS, and ALK Mutations in Non-Small Cell Lung Cancer. Eur Radiol [Internet]. 2015; Available from: http://dx.doi.org/10.1007/s00330-015-3814-0

31. Liu Y, Kim J, Balagurunathan Y, Li Q, Garcia AL, Stringfield O, et al. Radiomic Features Are Associated With EGFR Mutation Status in Lung Adenocarcinomas. Clin Lung Cancer [Internet]. Elsevier; 2016; Available from: http://dx.doi.org/10.1016/j.cllc.2016.02.001

32. Guo W, Li H, Zhu Y, Lan L, Yang S, Drukker K, et al. Prediction of clinical phenotypes in invasive breast carcinomas from the integration of radiomics and genomics data. J Med Imaging (Bellingham). medicalimaging.spiedigitallibrary. ...; 2015;2:041007.

33. Yoon HJ, Sohn I, Cho JH, Lee HY, Kim J-H, Choi Y-L, et al. Decoding Tumor Phenotypes for ALK, ROS1, and RET Fusions in Lung Adenocarcinoma Using a Radiomics Approach. Medicine . ncbi.nlm.nih.gov; 2015;94:e1753.

34. Yip SS, Kim J, Coroller T, Parmar C, Rios Velazquez E, Huynh E, et al. Associations between somatic mutations and metabolic imaging phenotypes in non-small cell lung cancer. J Nucl Med [Internet]. 2016; Available from: http://dx.doi.org/10.2967/jnumed.116.181826

35. Oesper L, Satas G, Raphael BJ. Quantifying tumor heterogeneity in whole-genome and whole-exome sequencing data. Bioinformatics. 2014;30:3532-40.

36. Weinstein JN, Collisson EA, Mills GB, Shaw KR, Ozenberger BA, Ellrott K, et al. The Cancer Genome Atlas Pan-Cancer analysis project. Nat Genet. 2013;45:1113-20.

37. Wang H, Schabath MB, Liu Y, Stringfield O, Balagurunathan Y, Heine JJ, et al. Association Between Computed Tomographic Features and Kirsten Rat Sarcoma Viral Oncogene Mutations in Patients With Stage I Lung Adenocarcinoma and Their Prognostic Value. Clin Lung Cancer. 2016;17:271-8.

38. Liu Y, Kim J, Qu F, Liu S, Wang H, Balagurunathan Y, et al. CT Features Associated with Epidermal Growth Factor Receptor Mutation Status in Patients with Lung Adenocarcinoma. Radiology. 2016;280:271-80.

39. Park J, Kobayashi Y, Urayama KY, Yamaura H, Yatabe Y, Hida T. Imaging Characteristics of Driver Mutations in EGFR, KRAS, and ALK among Treatment-Naïve Patients with Advanced Lung Adenocarcinoma. PLoS One. 2016;11:e0161081.

40. Program, National Cancer Institute Cancer Imaging. Quantitative Imaging for Evaluation of Responses to Cancer Therapies: Quantitative Imaging Network (QIN) [Internet]. 2013. Available from: http://imaging.cancer.gov/programsandresources/specializedinitiatives/qin

41. Gerlinger M, Rowan AJ, Horswell S, Larkin J, Endesfelder D, Gronroos E, et al. Intratumor heterogeneity and branched evolution revealed by multiregion sequencing. N Engl J Med. 2012;366:883-92. 
42. Querings S, Altmuller J, Ansen S, Zander T, Seidel D, Gabler F, et al. Benchmarking of mutation diagnostics in clinical lung cancer specimens. PLoS One. 2011;6:e19601.

43. Nishino M, Cardarella S, Dahlberg SE, Jackman DM, Ramaiya NH, Hatabu H, et al. Radiographic assessment and therapeutic decisions at RECIST progression in EGFR-mutant NSCLC treated with EGFR tyrosine kinase inhibitors. Lung Cancer. 2013;79:283-8.

44. Song C, Chen S, Yibin L, Rachel F, Alexander M, Harvey M, et al. Elimination of unaltered DNA in mixed clinical samples via nuclease-assisted minor-allele enrichment. Nucleic Acids Res. 2016;gkw650.

45. MacConaill LE, Garcia E, Shivdasani P, Ducar M, Adusumilli R, Breneiser M, et al. Prospective enterpriselevel molecular genotyping of a cohort of cancer patients. J Mol Diagn. 2014;16:660-72.

46. Brastianos PK, Horowitz PM, Santagata S, Jones RT, McKenna A, Getz G, et al. Genomic sequencing of meningiomas identifies oncogenic SMO and AKT1 mutations. Nat Genet. Nature Publishing Group, a division of Macmillan Publishers Limited. All Rights Reserved.; 2013/03//print;45:285-9.

47. MacConaill LE, Campbell CD, Kehoe SM, Bass AJ, Hatton C, Niu L, et al. Profiling Critical Cancer Gene Mutations in Clinical Tumor Samples. PLoS One. Public Library of Science; 2009;4:e7887.

48. Zhao B, James LP, Moskowitz CS, Guo P, Ginsberg MS, Lefkowitz RA, et al. Evaluating variability in tumor measurements from same-day repeat CT scans of patients with non-small cell lung cancer. Radiology. 2009;252:263-72.

49. Lê, S., Josse, J., Husson, F. FactoMineR: An R Package for Multivariate Analysis. J Stat Softw. 2008;25(1):1-18.

50. Benjamini Y, Hochberg Y. Controlling the False Discovery Rate: A Practical and Powerful Approach to Multiple Testing. J R Stat Soc Series B Stat Methodol. Blackwell Publishing for the Royal Statistical Society; 1995;57:289-300.

51. Schroder MS, Culhane AC, Quackenbush J, Haibe-Kains B. survcomp: an R/Bioconductor package for performance assessment and comparison of survival models. Bioinformatics. 2011;27:3206-8.

52. Parmar C, Grossmann P, Bussink J, Lambin P, Aerts HJWL. Machine Learning methods for Quantitative Radiomic Biomarkers. Sci Rep. Macmillan Publishers Limited; 2015;5:13087.

53. Brown G, Pocock A, Zhao M-J, Luján M. Conditional likelihood maximisation: a unifying framework for information theoretic feature selection. J Mach Learn Res. 2012;13:27-66.

54. Breiman L. Random forests. Mach Learn. 2001;45:5-32.

55. Wu W, Parmar C, Grossmann P, Quackenbush J, Lambin P, Bussink J, et al. Exploratory Study to Identify Radiomics Classifiers for Lung Cancer Histology. Front Oncol. 2016;6:71. 



\section{Chapter}

\section{Associations between somatic mutations and metabolic imaging phenotypes in non-small cell lung cancer}

Published in Journal of Nuclear Medicine, October 2016

Associations between somatic mutations and metabolic imaging phenotypes in non-small cell lung cancer

Stephen SF Yip ", John Kim", Thibaud P. Coroller, Chintan Parmar, Emmanuel Rios Velazquez, Elizabeth Huynh, Raymond H. Mak and Hugo J.W.L. Aerts 


\section{ABSTRACT}

Purpose. Positron emission tomography (PET)-based radiomics has been used to noninvasively quantify the metabolic tumor phenotypes; however, little is known about the relationship between these phenotypes and underlying somatic mutations. This study assessed the association and predictive power of ${ }^{18} \mathrm{~F}$-fluorodoxyglucose PET $\left({ }^{18} \mathrm{~F}\right.$-FDG PET)-based radiomic features for somatic mutations in non-small cell lung cancer (NSCLC) patients.

Methods. 348 NSCLC patients underwent diagnostic ${ }^{18}$ F-FDG PET scans and were tested for genetic mutations. 13\% (44/348) and 28\% (96/348) of patients were found to harbor an epidermal growth factor receptor (EGFR) or Kristen rat sarcoma viral (KRAS) mutations, respectively. We evaluated 21 imaging features: 19 independent radiomic features quantifying phenotypic traits and 2 conventional features (metabolic tumor volume and maximum standard uptake value. The association between imaging features and mutation status (e.g. EGFR+ vs. EGFR-) was assessed using the Wilcoxon rank-sum test. The ability of each imaging feature to predict mutation status was evaluated by the area under the receiver operating curve (AUC) and its significance was compared to a random guess ( $A \cup C=0.5$ ) using the Noether's test. All p-values were corrected for multiple hypothesis testing by controlling the false discovery rate (FDR Wilcoxon, $\left._{\text {, }} F R_{\text {Noether }}\right)$ with a significance threshold of $10 \%$.

Results. Eight radiomic features and both conventional features were significantly associated with EGFR mutation status $\left(F D R_{\text {Wilcoxon }}=0.01-0.10\right)$. One radiomic feature (normalized inverse difference moment) outperformed all other features in predicting EGFR mutation status ( $E G F R+$ vs $E G F R-, A U C=0.67, F D R_{\text {Noether }}=0.0032$ ), as well as differentiating between KRAS+ and EGFR+ (AUC=0.65, FDR noether $=0.05)$. None of the features were associated with or predictive of KRAS mutation status (KRAS+ vs. KRAS-, AUC $=0.50-$ $0.54)$.

Conclusions. Our results indicate that EGFR mutations may drive different metabolic tumor phenotypes that are captured in PET images, whereas KRAS mutated tumors do not. This proof-of-concept study sheds light on genotype-phenotype interactions, using radiomics to capture and describe the phenotype, and may have potential for developing non-invasive imaging biomarkers for somatic mutations 


\section{INTRODUCTION}

Non-small cell lung cancer (NSCLC) accounts for over $80 \%$ of lung cancers and affects over 220,000 people in the United States. It is the leading cause of cancer-related death, with a 5 -year survival rate of only $15 \%$ (1). Advances in cancer genomics have demonstrated that NSCLC is driven by somatic mutations in key oncogenes, such as epidermal growth factor receptor (EGFR) and Kristen rat sarcoma viral (KRAS) $(2,3)$. These discoveries have led to the use and regulatory approval of EGFR-specific tyrosine kinase inhibitors for treatment of patients who harbor an EGFR mutation (4-6). Furthermore, KRAS mutants have been found to respond poorly to both tyrosine kinase inhibitors and conventional cisplatin-based chemotherapy, resulting in poor treatment outcomes $(7,8)$. Therefore, given the driving force of mutation status in the efficacy of NSCLC therapies, identification of mutation status is crucial for selecting the most effective treatments, and ultimately, dictating patient outcomes. The current standard of care uses molecular testing to identify mutation status based on biopsies of tumor tissue or surgical resection; however, molecular testing can be limited by invasive procedures, long processing times, sampling error, and tissue samples are not always readily available (9-11).

Non-invasive ${ }^{18} \mathrm{~F}$-fluorodoxyglucose positron emission tomography/computed tomography $\left({ }^{18} \mathrm{~F}\right.$-FDG PET/CT) is increasingly used for imaging of glucose metabolism, and is part of the standard initial work-up for NSCLC patients in the United States $(12,13)$. Furthermore, the metabolic tumor phenotype captured in PET images may be an indication of the underlying biology associated with somatic mutations. For example, a mutation in EGFR activates the Akt signaling pathway, which promotes glucose consumption in tumor cells for their continuous growth and survival $(14,15)$. In addition, increased glucose transport and glycolysis have been observed in KRAS-mutated colorectal and pancreatic cancer cell lines $(16,17)$. Thus, previous studies have investigated the associations between these mutations and standardized uptake value (SUV) measures from PET images; however, there have been conflicting findings (18-22). These inconsistent reports may be due to the fact that simple SUV measures fail to capture the spatial relationships between image voxels, which may be more informative of the biology of these mutations and describes the degree of tumor heterogeneity $(23,24)$. Highly heterogeneous tumors are often associated with poor prognosis since resistance to therapy can develop in subpopulations of tumor cells $(25,26)$. Therefore, accurate quantification of tumor heterogeneity from PET images may provide important information for the identification of mutation status and precision medicine. Heterogeneity in the tumor phenotype can be quantitatively described through radiomic features $(23,27,28)$, which use advanced mathematical models to quantify the spatial relationship between image voxels (29). 
In this proof-of-concept study, we assessed the associations between PET radiomic features with somatic mutations and their ability to predict mutation status in 348 NSCLC patients. To our knowledge, this is the first study to investigate the relationship between somatic mutations and the metabolic phenotype, which may provide valuable information for developing a non-invasive imaging biomarkers to complement molecular testing in determining mutation status.

\section{METHODS AND MATERIALS}

\section{Patient imaging}

This retrospective study was conducted under a Dana-Farber/Harvard Cancer Center Institutional Review Board approved protocol. This study included 348 patients with NSCLC who received a diagnostic ${ }^{18}$ F-FDG PET/CT scan prior to treatment between September 2003 and December 2013. Patient characteristics are shown in Table 1.

Patients were injected with 9.3-24.9 $\mathrm{mCi}$ of ${ }^{18} \mathrm{~F}-\mathrm{FDG}$ and scanned approximately $65 \mathrm{~min}$ after injection on a GE scanner (GE Healthcare, Waukesha, WI), Siemens Biograph (Siemens AG, Erlangen, Germany), or GEMINI TF (Philips Medical Systems, Cleveland, $\mathrm{OH}$ ) PET/CT scanner (Table 1). Attenuation correction was performed on the PET images using the corresponding CT images. The acquisition time was 3-5 min per bed position for a whole-body scan.

\section{Mutation status analysis and patient classification}

Tissue samples of primary tumors were acquired through biopsy or surgical resection. Somatic mutations were tested using a polymerase chain reaction-based method or PROFILE Oncomap (30). The nucleotide sequence encoding the kinase domain (exons 18-24) of EGFR and exons 2-3 of KRAS were analyzed by polymerase chain reactionbased method and capillary gel electrophoresis. PROFILE Oncomap is a mass spectrometry genotyping technique that analyses over 470 unique mutations in 41 oncogenes. $51 \%(178 / 348)$ of patients had their mutation status identified by PCR and $49 \%$ (170/348) using PROFILE Oncomap.

Of the 348 patients that were tested for EGFR mutations, 44 patients tested positively for an EGFR mutation (EGFR+), while 304 patients were EGFR negative (EGFR-). Among the 44 EGFR+ patients, $19(43 \%)$ and 2 (5\%) had a L858R or L861Q substitution mutation in exon 21, respectively. 2 (5\%) had a G719C and 2 (5\%) had a G719S substitution mutation in exon 18. A deletion mutation in exon 19 was found in 19 (43\%) EGFR+ patients. 317 patients were tested for KRAS mutations. While 96 patients harbored a KRAS 
mutation (KRAS+), 221 patients were KRAS-. Of the 96 KRAS+ patients, 47 (49\%), 48 (50\%), and 1 (1\%) patient(s) had a substitution mutation in exon 1, 2, or 3, respectively.

185 patients tested negatively for EGFR and KRAS mutations (EGFR- and KRAS-). 31 patients were only tested for an EGFR mutation, but not KRAS, due to lack of medical necessity. In this study, we performed three comparisons: EGFR+ vs. EGFR-, EGFR+ vs. KRAS+, and KRAS+ vs. KRAS-. A subset analysis was also performed on patients with adenocarcinoma histology (251 patients). Of these 251 patients, 36 and 84 patients harbored EGFR and KRAS mutations, respectively.

\section{PET feature extraction and selection}

The metabolic tumor volume (MTV) was delineated on PET images with a fixed SUV threshold of 2.5. A nuclear medicine board-certified radiologist (J.K.) was blinded from the mutation data and reviewed the delineations of all 348 MTVs. All radiomic features were implemented in MATLAB (The Mathworks Inc., Natick, MA) and computed within the MTV. Prior to computation of the radiomic features, the image voxel intensities were resampled into equally spaced bin widths of 0.1 (31).

In total, 68 radiomic features were extracted from the PET images, including 7 shape features (29), 13 histogram-based features (29) and 48 texture features. The texture features included 22 gray level co-occurrence matrix (32), 11 run length matrix (33), 10 size zone matrix (34), 5 neighborhood gray-tone difference matrix (35) features (Sup-

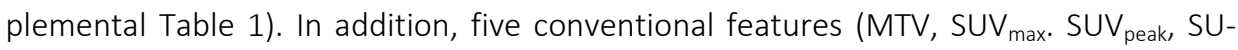
$\left.V_{\text {mean }}, S_{U} V_{\text {total }}\right)$ were computed for comparison with the radiomic features.

Table 1. Patient characteristics.

\begin{tabular}{lllc}
\hline & EGFR+ & KRAS + & Total \\
\hline Number of Patients & 44 & 96 & 348 \\
Sex & & & \\
$\quad$ Male & $9(20 \%)$ & $34(35 \%)$ & $134(39 \%)$ \\
Female & $35(80 \%)$ & $63(65 \%)$ & $214(61 \%)$ \\
Median age (year) & 61 & 67 & 65 \\
Range & $(34-88)$ & $(46-84)$ & $(34-93)$ \\
Ethnicity & & & \\
Caucasian & $38(86 \%)$ & $91(95 \%)$ & $316(91 \%)$ \\
African American & $2(5 \%)$ & $4(4 \%)$ & $21(6 \%)$ \\
Hispanic & $2(5 \%)$ & $1(1 \%)$ & $4(1 \%)$ \\
Asian & $2(5 \%)$ & $0(0 \%)$ & $6(2 \%)$ \\
Not reported & $0(0 \%)$ & $0(0 \%)$ & $1(0 \%)$ \\
Smoking history & & & $286(82 \%)$ \\
Current/Former & $24(55 \%)$ & $93(97 \%)$ & $62(18 \%)$ \\
Never & $20(45 \%)$ & $3(3 \%)$ & \\
\hline
\end{tabular}




\begin{tabular}{|c|c|c|c|}
\hline & EGFR+ & KRAS+ & Total \\
\hline \multicolumn{4}{|l|}{ Primary site } \\
\hline Upper lobe & $28(64 \%)$ & 57 (59\%) & $215(62 \%)$ \\
\hline Middle lobe & $1(2 \%)$ & $12(13 \%)$ & $27(8 \%)$ \\
\hline Lower lobe & $14(32 \%)$ & $24(25 \%)$ & $98(28 \%)$ \\
\hline Overlapping lesion & $1(2 \%)$ & $1(1 \%)$ & $2(1 \%)$ \\
\hline \multicolumn{4}{|l|}{ Clinical stage } \\
\hline । & $15(34 \%)$ & $30(31 \%)$ & $102(29 \%)$ \\
\hline$\|$ & $4(9 \%)$ & $11(11 \%)$ & $44(13 \%)$ \\
\hline III & $16(36 \%)$ & $36(38 \%)$ & $144(42 \%)$ \\
\hline IV & $9(21 \%)$ & $19(20 \%)$ & $58(16 \%)$ \\
\hline \multicolumn{4}{|l|}{ Tumor grade } \\
\hline Well-differentiated & $4(9 \%)$ & $8(8 \%)$ & 32 (9\%) \\
\hline Moderately-differentiated & $19(43 \%)$ & $25(26 \%)$ & $107(31 \%)$ \\
\hline Poorly-differentiated & $13(30 \%)$ & $36(38 \%)$ & $126(36 \%)$ \\
\hline Not determined & $8(18 \%)$ & $27(28 \%)$ & $83(24 \%)$ \\
\hline \multicolumn{4}{|l|}{ Histology } \\
\hline Adenocarcinoma & $36(82 \%)$ & $84(88 \%)$ & $251(72 \%)$ \\
\hline Squamous cell carcinoma & $0(0 \%)$ & $2(2 \%)$ & $31(9 \%)$ \\
\hline Non-small cell lung carcinoma NOS & $7(16 \%)$ & $10(10 \%)$ & $60(17 \%)$ \\
\hline Other & $0(0 \%)$ & $0(0 \%)$ & $7(2 \%)$ \\
\hline No pathology report & $1(2 \%)$ & $0(0 \%)$ & $3(1 \%)$ \\
\hline \multicolumn{4}{|l|}{ PET/CT Scanners } \\
\hline \multicolumn{4}{|l|}{ GE Discovery } \\
\hline ST & $13(30 \%)$ & $21(22 \%)$ & $81(23 \%)$ \\
\hline STE & $16(36 \%)$ & $36(37 \%)$ & $135(39 \%)$ \\
\hline LS & $2(5 \%)$ & $3(3 \%)$ & $12(3 \%)$ \\
\hline $\mathrm{RX}$ & $6(14 \%)$ & $13(14 \%)$ & $46(13 \%)$ \\
\hline \multicolumn{4}{|l|}{ Siemens Biograph } \\
\hline $\mathrm{mCT}$ & $5(11 \%)$ & $15(16 \%)$ & $43(13 \%)$ \\
\hline True Point & $1(2 \%)$ & $3(3 \%)$ & $9(3 \%)$ \\
\hline Phillips Gemini TF & $0(0 \%)$ & $5(5 \%)$ & $14(4 \%)$ \\
\hline Unspecified & $1(2 \%)$ & $0(0 \%)$ & $8(2 \%)$ \\
\hline
\end{tabular}

"Other" histology includes carcinoid tumor $(n=4)$, adenosquamous carcinoma $(n=4)$, sarcomatoid carcinoma $(n=2)$, undefined non-small cell lung cancer (NSCLC) $(n=3)$ and mixed NSCLC and SCLC $(n=1)$.

\section{Radiomics feature selection}

The Spearman's correlation coefficient $(R)$ was used to assess the correlation between all radiomic and conventional features in $\mathrm{R}$ software (version 3.2). Feature pairs with a $|R|>0.95$ were considered to be highly correlated and likely to provide redundant rather than complementary information about the mutation status. Features with the highest average $|R|$ were excluded. As a result, 21 features (19 radiomic and 2 conventional) were included in the analysis, which included 1 shape feature, 3 histogrambased features, and 15 texture features, and 2 conventional features. The selected features are shown in Table 2. All other 51 features were correlated to at least one of 
these 21 features with R>0.95 (Supplemental Figs. 1 and 2). Pearson's correlation coefficient was used to assess the correlation between the selected 21 features, which ranged from 0.05-0.95 as shown in Supplemental Fig. 2.

\section{Data analysis}

The association of PET features with mutation status was evaluated by comparing the distribution of each feature within the following groups: 1) EGFR+ vs. EGFR-, 2) KRAS+ vs. KRAS-, and 3) EGFR+ vs. KRAS+. The Wilcoxon rank-sum test was used to determine if there was a significant difference in the feature values between each mutation status. All $\mathrm{p}$-values were corrected for multiple hypothesis testing using the Benjamini-Hochberg method (false discovery rate) (36) (FDR wilcoxon) with a significance threshold of $10 \%$.

Univariate analysis was performed with R software (version 3.2) using the "pROC" and "survcomp" packages from Bioconductor (37). We evaluated the performance of each feature in classifying patients according to their mutation status (i.e. EGFR+ from EGFR-, KRAS+ from KRAS-, and EGFR+ from KRAS+). The performance was quantified using the $A \cup C$. The AUC is interpreted as the probability of correctly classifying the patients into different mutation categories and ranges from 0.5 to 1.0, where a value of 0.5 indicates a random guess and a value of 1.0 indicates a perfect classification. Noether's test was used to determine if the AUCS were significantly greater than 0.5. P-values were FDR corrected $\left(F_{\text {Noether }}\right)$ with a significance threshold of $10 \%$.

To assess if the radiomic features significantly outperformed the conventional features in predicting mutation status, the receiver operating characteristic (ROC) curves of the radiomic features with the highest AUCs were compared with the ROC curves of MTV and SUV $V_{\max }$ using a bootstrap test (38). P-values less than 0.05 were consisted statistically significant.

\section{RESULTS}

This study assessed the association of PET-based radiomic features with NSCLC mutation status and evaluated their ability to predict mutation status in a large patient cohort. Of 348 patients who were tested for somatic mutations, 13\% (44/348) of patients were EGFR+, while $28 \%$ (96/348) of patients were KRAS+ (Table 1). The majority of patients in the total cohort were female (61\%), Caucasian (91\%), current/former smokers (82\%), and had adenocarcinoma (72\%). Approximately $40 \%$ and $60 \%$ of patients in the total cohort were overall Stage I/II and Stage III/IV, respectively. We observed that patients who harbored an EGFR mutation (EGFR+) generally had smaller and more compact tumors with lower 18F-FDG uptake than EGFR- (Figs. 1 and 2). 
Table 2. Description of the selected PET-based features that were included in the analysis. A run in the run length matrix is a string of the consecutive voxels having identical SUV values in a pre-defined direction. A zonein the size zone matrix is a size of a 3D volume consisting of identical SUV values.

\begin{tabular}{|c|c|c|}
\hline Feature Type & Feature & Description \\
\hline \multirow[t]{2}{*}{ Conventional } & Metabolic Tumor Volume (MTV) & $\begin{array}{l}\text { Volume of the tumor region with SUV > } \\
2.5\end{array}$ \\
\hline & $\begin{array}{l}\text { Maximum Standardized Uptake } \\
\text { Values (SUVmax) }\end{array}$ & $\begin{array}{l}\text { Maximum SUV of the voxels within the } \\
\text { tumor region }\end{array}$ \\
\hline Shape & Compactness & $\begin{array}{l}\text { How closely and firmly united the tumor } \\
\text { is }\end{array}$ \\
\hline \multirow[t]{3}{*}{ Histogram } & $\begin{array}{l}\text { Minimum Standardized Uptake Value } \\
\text { (SUVmin) }\end{array}$ & $\begin{array}{l}\text { Minimum SUV of the voxels within the } \\
\text { tumor region }\end{array}$ \\
\hline & Skewness & $\begin{array}{l}\text { Measures the degree of asymmetry of } \\
\text { the tumor SUV distribution }\end{array}$ \\
\hline & Kurtosis & $\begin{array}{l}\text { Measures the flatness of the tumor SUVS } \\
\text { distribution (or how heavy-tailed or light } \\
\text { tailed the distribution is) relative to a } \\
\text { normal distribution }\end{array}$ \\
\hline $\begin{array}{l}\text { Gray Level } \\
\text { Co-occurrence Matrix }\end{array}$ & Maximum Probability (MaxProb) & $\begin{array}{l}\text { Determines how likely the most } \\
\text { predominant SUV voxel pair occurs }\end{array}$ \\
\hline \multirow[t]{4}{*}{ (GLCM) } & $\begin{array}{l}\text { Information Measure of Correlation } \\
\text { (InfoCorr) }\end{array}$ & $\begin{array}{l}\text { Measures the relationship between } \\
\text { tumor voxels }\end{array}$ \\
\hline & $\begin{array}{l}\text { Normalized Inverse Difference } \\
\text { (InvDiffnorm) }\end{array}$ & Measures tumor smoothness \\
\hline & $\begin{array}{l}\text { Normalized Inverse Difference } \\
\text { Moment } \\
\text { (InvDiffmomnor) }\end{array}$ & Measures tumor homogeneity \\
\hline & Inverse Variance (InverseVar) & $\begin{array}{l}\text { Inverse of the variability of the tumor } \\
\text { voxel SUVs }\end{array}$ \\
\hline $\begin{array}{l}\text { Gray Level Run Length } \\
\text { Matrix }\end{array}$ & Long Run Emphasis (LRE) & Measures coarse tumor textures \\
\hline \multirow[t]{2}{*}{ (GLRLM) } & Run Percentage (RunPct) & $\begin{array}{l}\text { Measures homogeneity of the tumor and } \\
\text { the distribution of runs in a specific } \\
\text { direction }\end{array}$ \\
\hline & $\begin{array}{l}\text { Long Run Low Gray Emphasis } \\
\text { (LRLGE) }\end{array}$ & $\begin{array}{l}\text { Measures the joint distribution of low } \\
\text { SUVs and long runs within the tumor }\end{array}$ \\
\hline $\begin{array}{l}\text { Gray Level Size Zone } \\
\text { Matrix }\end{array}$ & Small Zone Emphasis (SZE) & Measures fine tumor textures \\
\hline \multirow[t]{4}{*}{$(G L S Z M)$} & Large Zone Emphasis (LZE) & Measures coarse tumor textures \\
\hline & Size Zone Variability (SZV) & $\begin{array}{l}\text { Measures the similarity of the zones } \\
\text { throughout the tumor }\end{array}$ \\
\hline & $\begin{array}{l}\text { Low Gray Small Zone Emphasis } \\
\text { (LGSZE) }\end{array}$ & $\begin{array}{l}\text { Measures the joint distribution of low } \\
\text { SUVs } \\
\text { and small zones within the tumor }\end{array}$ \\
\hline & $\begin{array}{l}\text { Low Gray Large Zone Emphasis } \\
\text { (LGLZE) }\end{array}$ & $\begin{array}{l}\text { Measures the joint distribution of low } \\
\text { SUVs and large zones within the tumor }\end{array}$ \\
\hline $\begin{array}{l}\text { Neighborhood Gray } \\
\text { Tone Difference Matrix }\end{array}$ & Busyness & $\begin{array}{l}\text { Measures the change in SUV between } \\
\text { multiple tumor voxels and their } \\
\text { surroundings }\end{array}$ \\
\hline (NGTDM) & Complexity & $\begin{array}{l}\text { Measures the high level contents (e.g. } \\
\text { Edges and lines) }\end{array}$ \\
\hline
\end{tabular}


The total set of PET features extracted from the tumor volume was reduced to a small subset of descriptive features using an unsupervised selection method that reduced redundancy among the features. This method resulted in 21 features (19 radiomic and 2 conventional) that were analyzed for their associations with and predictive power for mutation status.

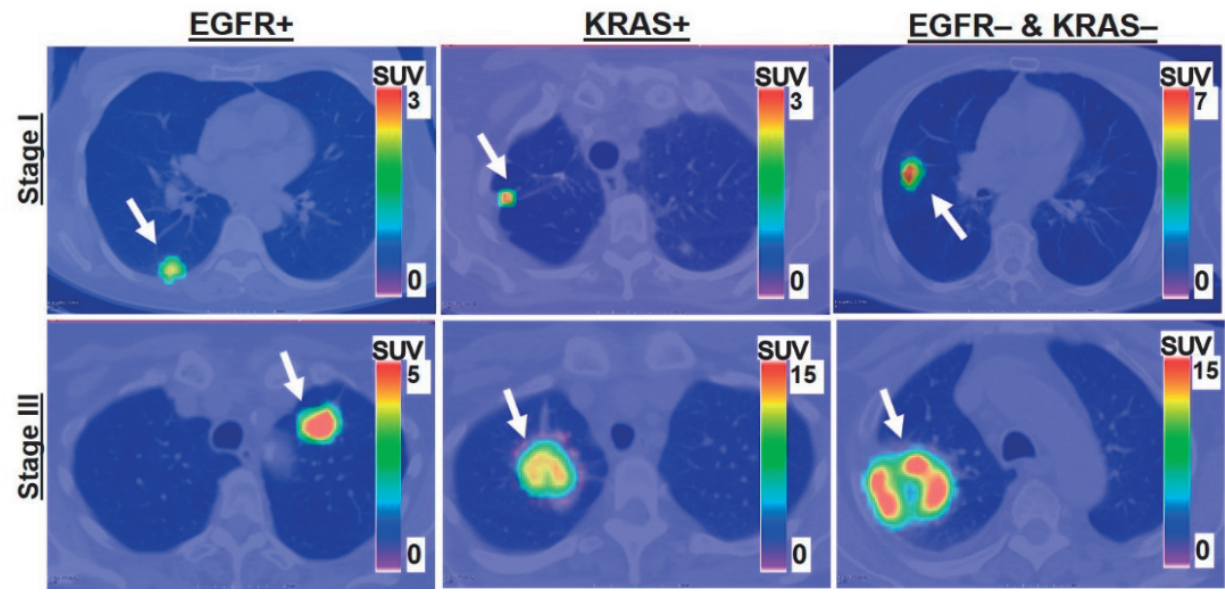

Figure 1. From left to right are patients with EGFR mutation, KRAS mutation, and EGFR- \& KRAS- tumors, respectively. Stage I and III tumors are shown in the top and bottom rows, respectively. Arrows indicate the locations of the lung tumors.

\section{Comparison of PET features between mutations}

To assess the associations between PET features and mutation status, we compared the distribution of values of each feature between the following groups: EGFR+ vs. EGFR-, EGFR+ vs. KRAS+, and KRAS+ vs. KRAS- (Fig. 2). A significant difference between the medians of the feature values for each mutation status indicated an association between the feature and mutation. Significant associations with 8 radiomic features (Compactness, SUVmin, MaxProb, InvDiffmomnor, Long-Run-Low-Gray-Emphasis, SizeZone-Variability, Low-Gray-Size-Zone-Emphasis, and Busyness), as well as both conventional features (MTV, SUVmax), and EGFR mutation status was observed (EGFR+ vs. EGFR-, FDRWilcoxon=0.01-0.10). A significant association was found in the radiomic feature normalized inverse difference moment (InvDiffmomnor) between EGFR+ and KRAS+ (FDRWilcoxon=0.08). None of the features were found to be significantly different between KRAS+ and KRAS- (FDRWilcoxon $\geq 0.92$ ). Compactness was significantly associated with the EGFR mutation (EGFR+ vs. wildtype (WT), FDRWilcoxon=0.08) (Supplemental Fig. 3). KRAS mutants demonstrated no distinctive imaging features. In particular, none of the PET features were significantly associated with the KRAS mutation (FDRWilcoxon $\geq 0.67$ ) (Supplemental Fig. 3). 


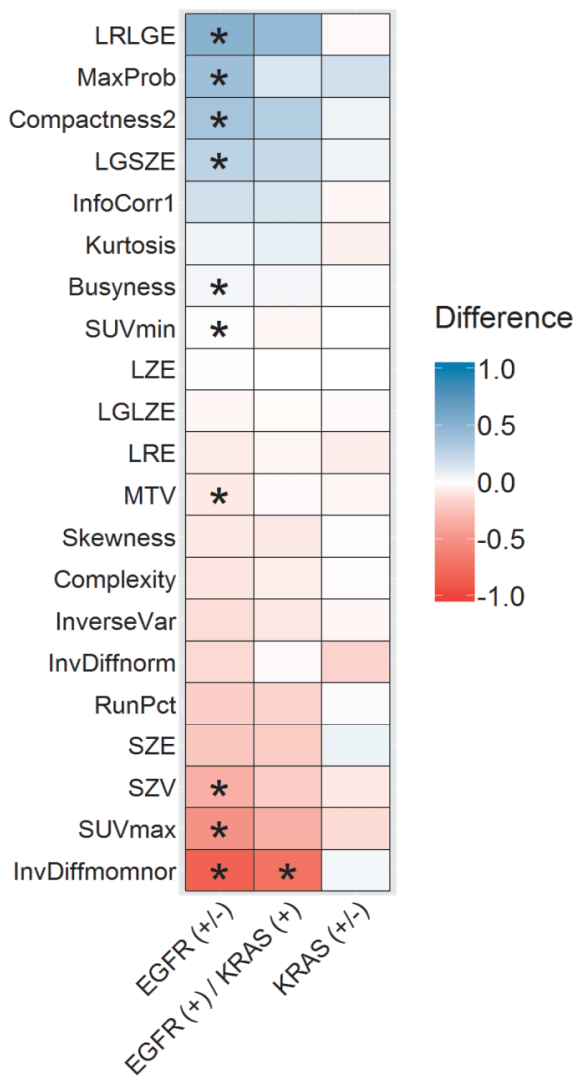

Figure 2. Comparison of PET features between mutation statuses. The Wilcoxon rank-sum test was used to determine if there was a significant difference in the PET feature between the mutation statuses. FDR wilcox- $_{\text {- }}$ ${ }_{\text {on }} \leq 0.10$ is indicated by *. The values of all PET features were normalized using a z-transformation. Entries in the columns of EGFR+ vs. EGFR-, EGFR+ vs. KRAS+, and KRAS+ vs. KRAS- represent the differences of the medians of the transformed measures. For example, in the EGFR+ vs EGFR-column, entry values $<0$ indicate that the median value of the PET feature for EGFR+ is lower than EGFR-.

\section{Prediction of mutation status}

The 21 PET features were evaluated for their ability to predict mutation status and were assessed by the AUC. 8 radiomic features (Compactness, SUV ${ }_{\min }$, MaxProb, InvDiffmomnor, Long-Run-Low-Gray-Emphasis, Size-Zone-Variability, Low-Gray-Size-Zone-Emphasis, and Busyness) and both conventional features (MTV, SUV max $_{\text {) }}$ ) were predictive of EGFR mutation status with AUCs ranging from 0.59-0.67 ( $\left.F D R_{\text {Noether }}=0.0032-0.09\right)$ (Fig. 3). The radiomic feature InvDiffmomnor $\left(A \cup C=0.67, F D R_{\text {Noether }}=0.0032\right)$ had the highest $A U C$ for discriminating EGFR+ from EGFR-, and Long-Run-Low-Gray-emphasis (FDR Noether $=0.06$ ) and Busyness $\left(F D R_{\text {Noether }}=0.09\right)$ had $A U C s$ of 0.59 . Five radiomic features, including 
Compactness, MaxProb, InvDiffmomnor, Size-Zone-Variability, and Low-Gray-Size-ZoneEmphasis, were able to significantly discriminate EGFR+ from the EGFR-KRAS wildtype (WT) (AUC=0.61-0.67, FDR Noether $=0.003-0.04$ ) (Supplemental Fig. 4). In addition, conventional features MTV and SUV $V_{\max }$ were significantly predictive of EGFR+ and WT differentiation ( $A \cup C=0.62-0.64, F D R_{\text {Noether }}=0.01-0.02$ ). InvDiffmomnor outperformed all other measures in EGFR mutation prediction.

Only one radiomic feature, InvDiffmomnor ( $A \cup C=0.65, F D R_{\text {Noether }}=0.05$ ) was able to significantly differentiate EGFR+ from KRAS+ (Fig. 3). In particular, none of the conventional PET features were able to differentiate EGFR+ from KRAS+ (AUC $=0.56-0.60$, $\left.F D R_{\text {Noether }} \geq 0.29\right)$. All other features had AUCs ranging from 0.50-0.61 (FDR Noether $\left.\geq 0.29\right)$ for EGFR+ and KRAS+ discrimination. None of the features were significantly predictive of KRAS mutation status (KRAS+ vs. KRAS-;AUC $<0.55$, FDR Noether $_{20.92)}$ (Fig. 3) or discriminating KRAS+ from WT (AUC=0.50-0.57, FDR Noether $_{20.65)}$ (Supplemental Fig. 4).

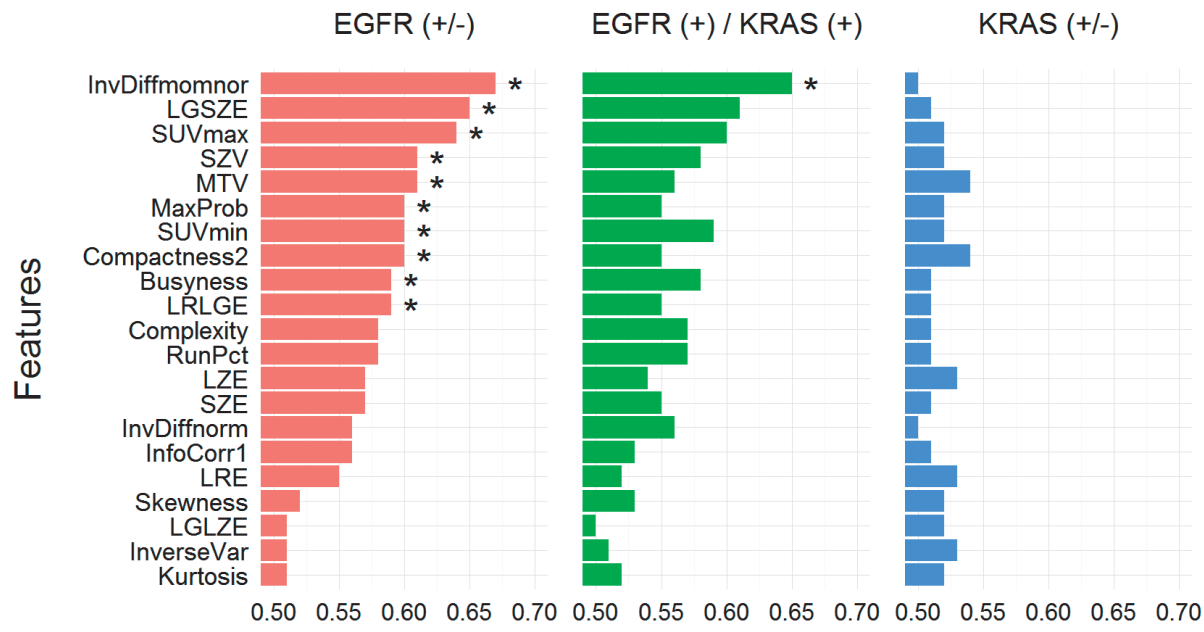

AUC

Figure 3. Area under the ROC curve $(A \cup C) .{ }^{*}$ indicates that the AUC is significantly $>0.50$ (random guessing) assessed with Noether's test $\left(F R_{\text {Noether }} \leq 0.10\right)$. Note that there are a large number of features bale to significantly predict EGFR positive tumors; however, they are not able to predict KRAS positive tumors.

The predictive performances of the significant radiomic features and conventional features for mutation status were compared directly to assess the value of radiomics over conventional features. The radiomic feature InvDiffmomnor significantly outperformed the conventional feature MTV in differentiating EGFR+ from EGFR- $(p=0.02)$, but did not significantly outperform the conventional feature $S U V_{\max }(p=0.14)$.

In patients with only adenocarcinoma histology, InvDiffmomnor (AUC=0.66, FDR $\mathrm{R}_{\mathrm{Ne}}$ other $=0.01$ ) was observed to outperform all other features (AUC $\leq 0.64, F D R_{\text {Noether }} \geq 0.03$ ) in 
differentiating EGFR+ from EGFR- (Supplemental Fig. 5), as observed with the full cohort. Adenocarcinoma EGFR mutants had significantly higher SUV $V_{\max }$ than its wildtypes $\left(F D R_{\text {Wilcoxon }}=0.06\right)$.

\section{DISCUSSION}

Identifying mutation status in NSCLC patients is an important component of selecting an optimal treatment plan for the patient. Clinically, a patient's mutation status is identified using molecular testing based on pathological examination of tumor tissues; however, this data may not always be readily available for all patients. We hypothesized that the genotype of tumors may be reflected in the phenotype that is captured in medical images. PET imaging is commonly used in the clinic for cancer management and can provide a non-invasive depiction of the tumor metabolic phenotype. We investigated the association between somatic mutations and 19 novel PET radiomic features and two conventional features (MTV and SUV $_{\max }$ ) in 348 NSCLC patients. We observed that radiomic features are strongly associated with EGFR mutation status and could significantly discriminate EGFR+ from EGFR-, and EGFR+ from KRAS+.

We found that EGFR mutants demonstrated distinctive imaging features compared to tumors without an EGFR mutation. For example, the metabolic region of EGFR+ tumors were smaller and had a lower SUV $\max$ than EGFR- tumors. Due to their low SUV uptake and smaller MTV, our results are consistent with the notion that EGFR mutants tend to be more indolent than other lung cancer types $(39,40)$. In addition, eight radiomic features were significantly associated with and predictive of EGFR mutation status, where InvDiffmomnor was the most predictive for EGFR mutation status and significantly outperformed the conventional measure MTV ( $p \leq 0.02)$. Furthermore, InvDiffmomnor was also found to outperform all other PET-based features in predicting EGFR mutations in patients with adenocarcinoma histology and was also predictive for differentiating EGFR+ from KRAS+ (supplemental Fig. 5). However, only five features were shown to significantly discriminate EGFR+ from WT (supplemental Figs. 3 and 4). This may be due to the fact that only 31 patients were tested only for EGFR mutations, but not KRAS mutations due to a lack of medical necessity. 74\% (23/31) of these patients were EGFR, while their KRAS mutation status was unknown. As it was unclear if those 23 patients were also KRAS-, they were excluded from the EGFR+ and WT differentiation analysis, which may explain why SUV $V_{\min }$, Busyness, and Long-Run-Low-Gray-Emphasis could significantly differentiate EGFR+ from EGFR-, but not EGFR+ from WT.

Our results showed that a lower SUV $\max$ was associated with EGFR+ tumors. Previous studies have also shown a substantial association between SUV $V_{\max }$ and EGFR mutation

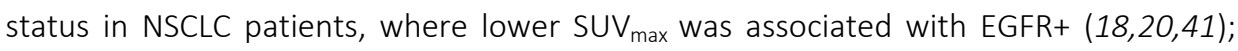
however, there have been conflicting conclusions with other studies. Conversely, stud- 
ies by Huang et al (2010) (21) and Lee et al (2013) (42) identified that higher SUV measures, such as SUV $V_{\max }$, was a strong predictor of EGFR+ tumors. In other studies, Lee et al. (2015) (43) and Chung et al. (2013) (44) found that none of their PET features (e.g. SUV $_{\max }$, MTV, and total glycolysis lesion) were significantly associated with or predicted EGFR mutation status.

The differences observed in these aforementioned studies may be attributed to the patient demographics for each study. Our study contained the largest patient cohort (348 patients) compared to the other studies (206 patients in Lee et al (2015) (43), 106 patients in Chung et al. (2013) (44), 77 patients in Huang et al. (2010) (21), and 214 patients in Lee et al. (2013) (42). Furthermore, the previous studies were from Asian institutions whereas our study was from a North American cohort, which resulted in over $90 \%$ of our patients being Caucasian (Table 1). Another study that had a predominantly Caucasian cohort was reported by Mak et al (2011) (18), who reported that a

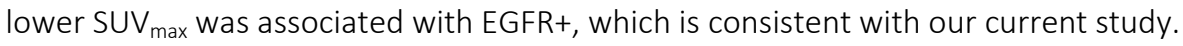

The frequency of EGFR mutations has been found to be substantially higher in Asian countries than Western countries (45). Therefore, the effect of ethnicity is reflected in the frequency of EGFR+ mutations in each cohort, where the previous studies had a higher percentage of EGFR+ mutants than in our study (23\% in the cohort of Lee et al. (2015) (43), 40\% in Chung et al. (2013) (44), 64\% in Huang et al. (2010) (21), and 24\% in Lee et al. (2013) (42) vs. only $13 \%$ in our study). However, despite the differences between each study, it is clear that the conclusions on using conventional PET-based features for prediction of mutation status remain debatable. The limitation of conventional PET features may be that they do not adequately quantify the tumor phenotype that reflects mutation status. Using a radiomics approach with greater descriptive power to quantify the tumor phenotype, we found that not only were many radiomic features predictive of EGFR mutation status, they could also be better predictors than conventional PET features. While this was an initial exploratory study to demonstrate that radiomics could be applied for mutation status prediction using PET images, these findings need to be further investigated in multiple large datasets acquired from different countries.

None of the conventional or radiomic features were associated with or predictive of KRAS mutation status (KRAS+ vs. KRAS-). We found that KRAS+ tumors tended to be bigger and had greater values in SUV measures than EGFR+ tumors, suggesting that KRAS+ tumors may be more aggressive (Figs. 1 and 2). Only one radiomic feature (InvDiffmomnor) could significantly discriminate KRAS+ from EGFR+, while other features, including conventional features, were unable to.

Other groups have also investigated the ability of conventional PET features to identify KRAS mutation status. Caicedo et al (2014) (19) investigated if SUV measures, including maximum, peak, and average SUV, could predict EGFR and KRAS mutation status in 102 
patients with NSCLC. Unlike our results, they found that KRAS+ tumors had significantly higher values in SUV measures than both EGFR mutants and KRAS-EGFR wildtypes. One reason for this difference between our study and their study may be because a proportion of the KRAS - tumors in our study were also EGFR+ (36 out of 221 (16\%) KRAStumors were EGFR mutants). In our analysis of KRAS+ and WT, we found that all the features performed poorly in prediction for the KRAS mutation. Moreover, we also found that the feature distributions between KRAS + and KRAS- were not significantly different for any of the PET features. However, these differences may also be attributed to the different cohort size since we investigated the association between PET imaging features and mutation status in 348 tumors, whereas the study by Caicedo et al (2014) (19) investigated 102 tumors.

There are several limitations of our study. First, due to the retrospective nature of the study, the imaging protocols were not standardized for all the patients, resulting in different acquisition and reconstruction parameters (Table 1). Although these differences may lead to variability in quantification of SUV (46-48), the distributions of scanners used for PET/CT image acquisition were similar for all patient groups (Table 1). Secondly, partial volume effects resulting from limited PET spatial resolution may lead to an underestimation of the metabolic tumor region and SUV measures (49). Furthermore, because of insufficient data acquisition and limited reconstruction techniques, respiratory motion can induce image blurring in the static (3D-PET) image acquisition, subsequently affecting the quantification of the PET features (50). Despite these limitations, many radiomic features were still found to be significantly associated with and predictive of EGFR mutation status. Whether PET standardization, correction of partial volume effects, and the use of respiratory-gated PET/CT imaging can improve the value of PET features in mutation identification needs to be further investigated. While this study focused on the use of radiomic features extracted from the untreated primary tumors for mutation prediction, in the future, it would be interesting to also investigate the predictive value of features extracted from metastatic lesions.

\section{CONCLUSIONS}

Tumor metabolic phenotypes that are driven by EGFR mutations could be quantified by radiomic features. Several radiomic features were strongly associated with the EGFR mutation status; in particular, InvDiffmomnor outperformed all other PET features (radiomic and conventional) in EGFR mutation prediction (EGFR+ vs. EGFR- and EGFR+ vs. KRAS+). All PET features were poorly associated with KRAS mutations, potentially indicating that tumors of KRAS mutants demonstrated weak distinctive imaging features.

Radiomic features extracted from diagnostic PET images have potential for identifying EGFR mutations in NSCLC. Our study may help develop an imaging biomarker to non- 
invasively identify EGFR mutation status using PET imaging to complement, but not to replace, molecular testing. Prospective studies with blinded mutation status and independent datasets will be needed to further validate the predictive power of the radiomic features. Furthermore, future studies will need to investigate how the EGFR mutation gives rise to certain phenotypic traits that are quantified by radiomic features.

\section{ACKNOWLEDGEMENTS}

The authors would like to acknowledge support from the National Institute of Health (Award Number U01CA190234 and U24CA194354) and research seed funding grant from the American Association of Physicists in Medicine. The authors would also like to thank the PROFILE team for their help with somatic mutation testing. 


\section{REFERENCES}

1. Siegel RL, Miller KD, Jemal A. Cancer statistics, 2015. CA Cancer J Clin. 2015;65:5-29.

2. Pao W, Girard N. New driver mutations in non-small-cell lung cancer. Lancet Oncol. 2011;12:175-180.

3. Pao W, Hutchinson KE. Chipping away at the lung cancer genome. Nat Med. 2012;18:349-351.

4. Paez JG, Jänne PA, Lee JC, et al. EGFR mutations in lung cancer: correlation with clinical response to gefitinib therapy. Science. 2004;304:1497-1500.

5. Maemondo M, Inoue A, Kobayashi K, et al. Gefitinib or chemotherapy for non-small-cell lung cancer with mutated EGFR. N Engl J Med. 2010;362:2380-2388.

6. Zhou C, Wu Y-L, Chen G, et al. Erlotinib versus chemotherapy as first-line treatment for patients with advanced EGFR mutation-positive non-small-cell lung cancer (OPTIMAL, CTONG-0802): a multicentre, open-label, randomised, phase 3 study. Lancet Oncol 2011;12:735-742.

7. Loriot Y, Mordant P, Deutsch E, Olaussen KA, Soria J-C. Are RAS mutations predictive markers of resistance to standard chemotherapy? Nat Rev Clin Oncol. 2009;6:528-534.

8. Pao W, Wang TY, Riely GJ, et al. KRAS mutations and primary resistance of lung adenocarcinomas to gefitinib or erlotinib. PLoS Med. 2005;2:e17.

9. Lindeman NI, Cagle PT, Beasley MB, et al. Molecular testing guideline for selection of lung cancer patients for EGFR and ALK tyrosine kinase inhibitors: guideline from the college of american pathologists, international association for the study of lung cancer, and association for molecular pathology. J Mol Diagn. 2013;15:415-453.

10. Pao W, Ladanyi M. Epidermal growth factor receptor mutation testing in lung cancer: searching for the ideal method. Clin Cancer Res. 2007;13:4954-4955.

11. Gerlinger M, Rowan AJ, Horswell S, et al. Intratumor heterogeneity and branched evolution revealed by multiregion sequencing. N Engl J Med. 2012;366:883-892.

12. Cuaron J, Dunphy M, Rimner A. Role of FDG-PET scans in staging, response assessment, and follow-up care for non-small cell lung cancer. Front Oncol. 2013;2:208.

13. Weber WA, Petersen V, Schmidt B, et al. Positron emission tomography in non-small-cell lung cancer: prediction of response to chemotherapy by quantitative assessment of glucose use. J Clin Oncol 2003;21:2651-2657.

14. Gan Y, Shi C, Inge L, Hibner M, Balducci J, Huang Y. Differential roles of ERK and Akt pathways in regulation of EGFR-mediated signaling and motility in prostate cancer cells. Oncogene. 2010;29:4947-4958.

15. Elstrom RL, Bauer DE, Buzzai M, et al. Akt stimulates aerobic glycolysis in cancer cells. Cancer Res. 2004;64:3892-3899.

16. Yun J, Rago C, Cheong I, et al. Glucose deprivation contributes to the development of KRAS pathway mutations in tumor cells. Science. 2009;325:1555-1559.

17. Ying $\mathrm{H}$, Kimmelman Alec $\mathrm{C}$, Lyssiotis Costas A, et al. Oncogenic Kras maintains pancreatic tumors through regulation of anabolic glucose metabolism. Cell. 2012;149:656-670.

18. Mak RH, Digumarthy SR, Muzikansky A, et al. Role of 18F-dluorodeoxyglucose positron emission tomography in predicting epidermal growth factor receptor mutations in non-small cell lung cancer. Oncologist. 2011;16:319-326.

19. Caicedo C, Garcia-Velloso M, Lozano M, et al. Role of [18F]FDG PET in prediction of KRAS and EGFR mutation status in patients with advanced non-small-cell lung cancer. Eur J Nucl Med. 2014;41:20582065.

20. Na II, Byun BH, Kim KM, et al. 18F-FDG uptake and EGFR mutations in patients with non-small cell lung cancer: A single-institution retrospective analysis. Lung Cancer. 2010;67:76-80.

21. Huang C-T, Yen R-F, Cheng M-F, et al. Correlation of F-18 fluorodeoxyglucose-positron emission tomography maximal standardized uptake value and EGFR mutations in advanced lung adenocarcinoma. Med Oncol. 2010;27:9-15.

22. Kawada K, Nakamoto $\mathrm{Y}$, Kawada M, et al. Relationship between 18F-dluorodeoxyglucose accumulation and KRAS/BRAF mutations in colorectal cancer. Clin Cancer Res. 2012;18:1696-1703. 
23. Cook GR, Siddique M, Taylor B, Yip C, Chicklore S, Goh V. Radiomics in PET: principles and applications. Clin Trans/ Imaging. 2014;2:269-276.

24. Rahim M, Kim S, So H, et al. Recent trends in PET image interpretations using volumetric and texturebased quantification methods in nuclear oncology. Nucl Med Mol Imaging. 2014;48:1-15.

25. Burrell RA, McGranahan N, Bartek J, Swanton C. The causes and consequences of genetic heterogeneity in cancer evolution. Nature. 2013;501:338-345.

26. Marusyk A, Almendro V, Polyak K. Intra-tumour heterogeneity: a looking glass for cancer? Nat Rev Cancer. 2012;12:323-334.

27. Henriksson E, Kjellen E, Wahlberg P, Ohlsson T, Wennerberg J, Brun E. 2-deoxy-2-[18F]fluoro-d-glucose uptake and correlation to intratumoral heterogeneity. Anticancer Res. 2007;27:2155-2159.

28. Yip SFS, Aerts JWLH. Applications and limitations of radiomics. Phys Med Biol. 2016;61:R150.

29. Aerts HJWL, Velazquez ER, Leijenaar RTH, et al. Decoding tumour phenotype by noninvasive imaging using a quantitative radiomics approach. Nat Commun. 2014;5:4006.

30. MacConaill LE, Garcia E, Shivdasani P, et al. Prospective enterprise-level molecular genotyping of a cohort of cancer patients. J Mol Diagn 2014;16:660-672.

31. Leijenaar RTH, Nalbantov G, Carvalho S, et al. The effect of SUV discretization in quantitative FDG-PET Radiomics: the need for standardized methodology in tumor texture analysis. Sci Rep. 2015;5:11075.

32. Haralick RM, Shanmugam K, Dinstein IH. Textural features for image classification. IEEE Trans Syst, Man, Cybern, Syst. 1973;SMC-3:610-621.

33. Galloway MM. Texture analysis using gray level run lengths. Comput Vision Graph. 1975;4:172-179.

34. Thibault G, Fertil B, Navarro C, et al. Shape and texture indexes application to cell nuclei classification Int J Pattern Recogn. 2013;27:1357002.

35. Amadasun M, King R. Textural features corresponding to textural properties. IEEE Trans Syst, Man, Cybern, Syst 1989;19:1264-1274.

36. Benjamini $Y$, Hochberg $Y$. Controlling the false discovery rate: a practical and powerful approach to multiple testing. J R Stat Soc Series B Stat Methodol. 1995;57:289-300.

37. Gentleman RC, Carey VJ, Bates DM, et al. Bioconductor: open software development for computational biology and bioinformatics. Genome Biol. 2004;5:R80-R80.

38. Hanley JA, McNeil BJ. A method of comparing the areas under receiver operating characteristic curves derived from the same cases. Radiology. 1983;148:839-843.

39. Chmielecki J, Foo J, Oxnard GR, et al. Optimization of dosing for EGFR-mutant non-small cell lung cancer with evolutionary cancer modeling. Sci Trans/ Med. 2011;3:90ra59-90ra59.

40. Rekhtman N, Ang DC, Riely GJ, Ladanyi M, Moreira AL. KRAS mutations are associated with solid growth pattern and tumor-infiltrating leukocytes in lung adenocarcinoma. Mod Pathol. 2013;26:1307-1319.

41. Choi Y-J, Cho B, Jeong Y, et al. Correlation between 18F-fluorodeoxyglucose uptake and epidermal growth factor receptor mutations in advanced lung cancer. Nucl Med Mol Imaging. 2012;46:169-175.

42. Lee $\mathrm{Y}$, Lee $\mathrm{H}-\mathrm{J}$, Kim YT, et al. Imaging characteristics of stage I non-small cell lung cancer on CT and FDGPET: relationship with epidermal growth factor receptor protein expression status and survival. Korean J Radiol. 2013;14:375-383.

43. Lee SM, Bae SK, Jung SJ, Kim CK. FDG uptake in non-small cell lung cancer is not an independent predictor of EGFR or KRAS mutation status: a retrospective analysis of 206 patients. Clin Nucl Med 2015;40:950-958.

44. Chung HW, Lee KY, Kim HJ, Kim WS, So Y. FDG PET/CT metabolic tumor volume and total lesion glycolysis predict prognosis in patients with advanced lung adenocarcinoma. J Cancer Res Clin Onco. 2013;140:8998.

45. Dearden S, Stevens J, Wu Y-L, Blowers D. Mutation incidence and coincidence in non small-cell lung cancer: meta-analyses by ethnicity and histology (mutMap). Ann Oncol 2013;24:2371-2376.

46. Boellaard R. Standards for PET image acquisition and quantitative data analysis. J Nucl Med. 2009;50:11S-20S.

47. Galavis PE, Hollensen C, Jallow N, Paliwal B, Jeraj R. Variability of textural features in FDG PET images due to different acquisition modes and reconstruction parameters. Acta Oncol. 2010;49:1012-1016. 


\section{Chapter 9}

48. Leijenaar RTH, Carvalho S, Velazquez ER, et al. Stability of FDG-PET radiomics features: an integrated analysis of test-retest and inter-observer variability. Acta Oncol. 2013;52:1391-1397.

49. Soret M, Bacharach SL, Buvat I. Partial-volume effect in PET tumor imaging. J Nucl Med 2007;48:932-945.

50. Yip S, McCall K, Aristophanous M, Chen AB, Aerts HJWL, Berbeco R. Comparison of texture features derived from static and respiratory-gated PET images in non-small cell lung cancer. PLOS ONE. 2014;9:e115510. 
General Discussion and Future Perspectives 



\section{Chapter}

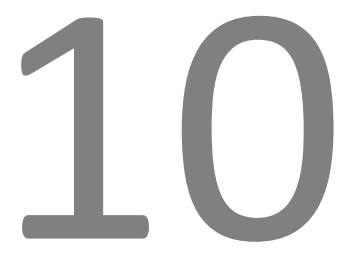

Conclusion and Perspective 



\section{GENERAL DISCUSSION}

Precision medicine is soon to be a standard in cancer care, evident through several initiatives sponsored by the US government such as Cancer Moonshot ${ }^{1}$ or Precision Medicine Initiative ${ }^{2}$. This highlights the need for deeper understanding of cancer mechanism and biology to potentially improve patient care and prognosis. The increasing volume of "omics" publications focusing on cancer (such as genomics ${ }^{3-5}$, proteomics ${ }^{6,7}$, metabolomics ${ }^{8,9}$, radiomics ${ }^{10-12}$ ) shows that public health decisions are supported by researchers findings and interest. Omics refers to the collective technologies used to explore the roles, relationships, and actions of various types of molecules that make up the cells of an organism. Besides adding more knowledge of tumor mechanism, these studies also highlight the tremendous complexity of cancer and the need for precision medicine. Creating a tool that gives clinicians the ability to do things such as tailoring patients' treatment, maximizing tumor response, and improving quality of life (by reducing dose or avoid surgery) would have a tremendous impact in standard state of cancer care.

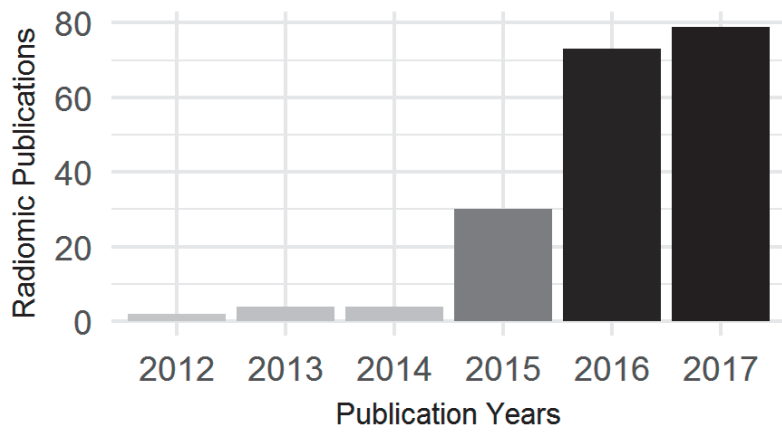

Figure 1. The barplot indicates the number of radiomic publication per years. Information was extract from PubMed for any entries including "radiomic" as in May 2017. After five months, 2017 already have more counts than 2016.

Precision medicine can be enabled using radiomic features, whose hypothesis is aims to non-invasively characterize the tumor phenotype using a high-throughput extraction of descriptive features from medical images. These features can be ultimately used to stratify patients regarding a specific risk (e.g. distant metastasis) or treatment response. Radiomics is a field rising in prominence as observed through PubMed (Figure 1). It has been attracting more and more researchers who published more papers, and thus quickly gaining momentum. Various studies have shown association between radiomic features and clinical outcomes ${ }^{13-16}$, somatic mutation ${ }^{17,18}$, and tumor response ${ }^{19-22}$. Additionally, stability and robustness of these features have been investigated ${ }^{23-26}$. However, like everything new, beginnings can be clumsy and disorganized regardless of best intentions. Therefore, this increasing interest comes with a price: a lack of stand- 
ardization and the presence of substandard practices. To establish radiomics future, several aspects need to be followed and disseminated (Figure 2).

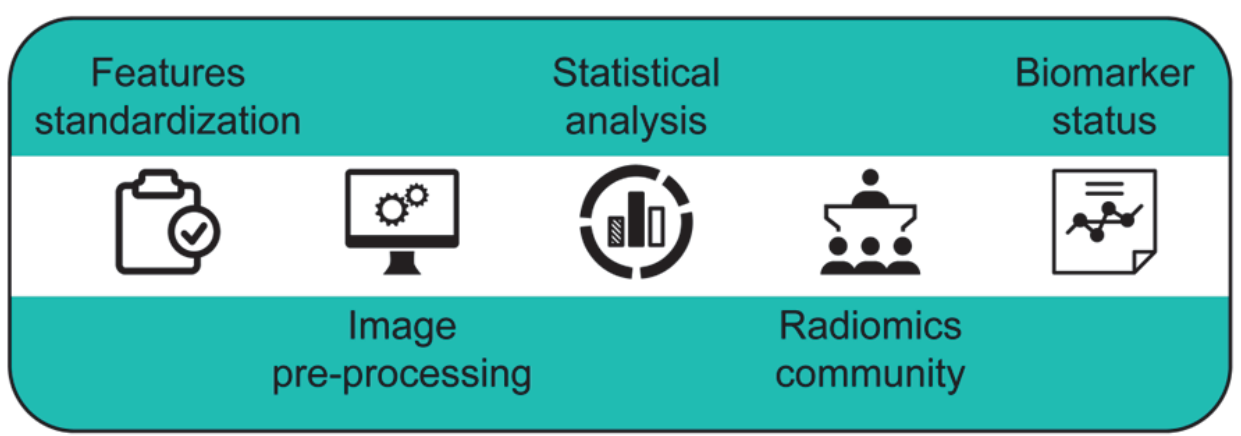

Figure 2. Illustration of important aspects in radiomic research.

Detailed section of each aspects can be found below:

1) Features standardization. It refers to features nomenclature and corresponding equations. Ideally, one feature has a unique name and equation. However, equation can end up having a different name, while one name can have two different equations depending on the research groups. Consequently, this makes communication about their work and sharing information difficult among researchers. This phenomenon is amplified by two facts: a lack of common and broadly used radiomics platform and institution's liberty to create their own features pipeline, thus defining and naming features at will. Features standardization is imperative to homogenize radiomics research and increase coherence between studies. It should also allow reproducibility by replicating values with consistent definition. On a brighter perspective, researchers are making great efforts, such as the Image Biomarker Initiative ${ }^{27}$, that try to standardize the field by developing a consensus that shares a large repository of feature definitions (including names and equations), so future groups can use as reference during code development. Further efforts are being made by sharing open source code that other researchers can use and even inspect, ensuring transparency in the results. Our laboratory currently contributes to these efforts through; for example we recently released a python open source radiomics features extraction script called PyRadiomics $^{28,29}$. Additionally, a group from MD Anderson released a radiomic platform $I B E X^{30}$ to improve collaboration between researchers.

2) Image pre-processing. This step is crucial for features extraction. Theoretically, each process is supposed to simplify the extraction by formatting images by various aspects such voxel spacing or intensity. Pre-processing steps commonly include: a) Voxel spacing resampling (for example $1 \times 1 \times 1 \mathrm{~mm}^{3}, 3 \times 3 \times 3 \mathrm{~mm}^{3}$ ), b) Interpolation method (linear, spline, quadratic), c) Image normalization (using Z- 
scores or removing outliers) or d) Voxel intensity binning. Pre-processing parameters have been shown to impact radiomic values, which in turn affect prediction from the features ${ }^{31}$. Various organizations such as the Quantitative Imaging Network (QIN) ${ }^{32}$ of the NIH is investigating future directions, by looking at phantom studies and standardized protocols for image acquisition. Thus, each parameters used in a study must be clearly described in the methods (or supporting documents) to provide readers with exhaustive information and allow them to replicate findings.

3) Statistical analysis. A majority of laboratories with interest in radiomics originated from medical physics. Therefore, a lack of knowledge in data science and biostatistics is often an issue that must be overcome. Adequate method and design must be selected. Multivariate analysis should include a validation set. If not available, a cross-validation to show performance of the model should be presented. Finally, multiple testing hypothesis correction (when testing a high number of hypothesis, by random some will appear significant) is an important yet most likely to be forgotten step in published studies. These practices should be disseminated to the community as standard. As a final safeguard, during peer-review process, it is the reviewer's responsibility to determine that submitted studies are satisfactorily designed and that claims are supported by statistical data.

4) Radiomics community. Annual meetings and conferences are a great way to connect, discuss, and share ideas. They could lead to fructuous collaborations, often only by sharing data to others and produce complex analysis design requiring various skills. Such collaborations ensure great visibility (high impact journals) and could quickly become landmark study. Multicentre studies are usually a requirement for acceptance to publish an "omics" study in a high impact journals. A model that is validated using multiple cohorts shows stronger evidences than from a unique centre (more variability in the data collection, thus harder to validate). It will also show greater transferability that is the most important when the goal of such model is to be used for clinical practice on a large scale.

5) Biomarker status. Unlike older and more investigated biomarkers such as those in genomics, biomarkers in radiomics have not yet accumulated the level of evidence needed for clinical applications ${ }^{33}$. In my opinion, it will only be reached by bringing a higher global standard and by enabling global collaborations. A major missing step for radiomics is prospective validation. Clinical trials investigating patients clustering based on their radiomics score will propel the field into the next stage, and hopefully radiomics will be clinically applicable and widely utilized. 


\section{Part 1: Prediction of lung tumor response to chemoradiation}

A common treatment for lung cancer is the combination of chemotherapy and radiation (called chemoradiation), followed by surgery a few weeks after completion. By controlling the tumor before resection, doctors expect a better survival chance. Although this is routine practice, no results have shown that patients have a better chance of survival with this approach ${ }^{34,35}$. This lack of benefit could be explained by two hypotheses: 1) having unnecessary surgery on a tumor that was cured with chemoradiation alone or 2) having weeks of chemoradiation against a resistant tumor that did not respond. The ability to predict either case at an early time point could be very valuable for doctors and allow precision medicine to improve patient care. For example, if a patient is very likely to respond to chemoradiation, surgery could be delayed to ensure it is needed. On the other hand, if a patient is unlikely to respond to chemoradiation, it should be shortened to reduce side effects and surgery could be performed earlier. Currently, only basic measurements such as tumor diameter or volume are extracted from images to assess its response.

In Chapter 2, we investigated a way to predict tumor response at time of diagnosis (prior to any treatment) using advanced imaging features (mathematical equations) that capture tumor traits $^{19}$. We showed significant links between tumor characteristics and its response to therapy such as tumor shape or its density patterns. We found that bulky tumors with a complex density pattern were more likely to resist chemoradiation treatment. In Chapter 3 we also showed that valuable information regarding tumor response could be extracted from the surrounding involved lymph nodes ${ }^{20}$. Interestingly, standard measures such as diameter or volume currently used in the clinic were not associated with tumor response to treatment. This information could be used to improve standard of care and help patients improve their outcome.

\section{Part 2: Lung tumor evolution after radiotherapy treatment}

Most lung cancer cases are treated with radiation ${ }^{36}$. As any treatment is disruptive to the tumor and could lead to a spread of the infected tissues across the patient body, chemotherapy is often used in conjunction to prevent it. Despite its use, a large number of patients (30-40\%) are likely to develop distant metastasis (cancer spreading outside the lung, generally the brain) and experience very low survival rates. If patients at risk (having a chance of developing distant metastasis or even local recurrence near the tumor) could be identified, clinicians could increase the dose of chemo and radiation and/or have a closer follow up to ensure good outcomes.

Through analysing medical images, (in this case computed tomography scans), we investigated the association between tumor characteristics and its evolution over time after treatment completion in Chapter 4 and Chapter 5 . We found that the tumor density is 
linked to its risk of spreading and local recurrence ${ }^{15}$. Moreover, we showed that combining clinical information that doctors already know with those image-based data improved the prediction of such patients at risk on a validation cohort ${ }^{13}$. Finally in Chapter 6 we shown that using 4D-CT images could add more information that free breathing images ${ }^{16}$ for any type of recurrences.

\section{Part 3: Tumor intrinsic nature ASSESSMENT}

Recent research has shown that non-small cell lung cancer (NSCLC), the most common type of lung cancer, is often caused by mutations in specific genes, including the commonly known ones that are called EGFR and KRAS. Approximately $15 \%$ of NSCLC patients have EGFR mutations and often benefit from a medication called tyrosine kinase inhibitor. Therefore, identification of these mutations is crucial for selecting the most effective treatment for these patients. The current method of identification relies on extracting tissue from the patient's tumor through biopsy (sample of tissue taken from the body through various ways, including surgical removal). However, this method is can be invasive to the patients, take a long time to return results, is subjected to errors, and sometimes tumor tissues are hard to extract from the body.

In chapter 7, we investigated meningioma tumour. Meningioma is the most frequent benign brain tumor in adults. Its management is driven by its grade, however there are currently no reliable way to predict it unless using biopsy. We use MRI image on 175 patients that underwent surgery. We found that radiographic (radiomic and semantic) features were individually associated with tumor grade using pre-surgery planning MR images. Additionally, combining radiomic and semantic features improved the classification of meningioma grade in the validation cohort.

Lastly, on chapter 8 we used CT images from over 750 patients in this multicentre study and mutation proven by biopsy. We found that radiomics features extracted from CT images were also abled to predict EGFR mutation but not KRAS. Knowing this information, doctors might be able to make treatment decision faster and without subjecting patients to invasive procedures. KRAS mutation predictors still need to be further investigated, as we did not find any in this study. In chapter 9, using a large number of PET scans, in 348 patients with NSCLC ${ }^{18}$, confirming findings in PET that EGFR was associated with distinct phenotype but no association were found with KRAS status.

\section{FUTURE PERSPECTIVES}

Future of radiomics lies in new computation tools and techniques. Big data ${ }^{37}$ is defined as using dataset so big that it cannot be stored or analysed on a single computer. Nowadays, from a single research institute perspective, we are far from this stage of imple- 
menting big data in radiomics due to data protection. Patients' protection laws make it difficult, for obvious reasons, to share or obtain information about their demographics, clinical characteristics, or treatment information. The cohort size will likely vary from a couple to thousands of patients maximum for most published studies. Relevant information for a thousand patients can easily be stored in a spreadsheet of maybe a few megabytes. Unlike medical data, web-based information such Amazon or Google) is exponentially increase over time. The storage and access must be design for this growth and thus need scalable tools (method created to handle very large amount of data) to analyse such dataset. While the needs of such methods is not there yet, it is important to think about the future and start a transition.

The necessity of big data framework might emerge from new and complex machine learning algorithms. The complexity comes with a cost of data for training. Deep learning $^{38}$ is a machine learning method that decomposes images in many layers with higher level of abstraction. The most known one is convolution neural network (CNN). Deep learning uses algorithms such as backpropagation to learn from errors and improve its mechanism by reinforcement learning. Deep learning has a major benefit over common radiomics approach: it doesn't require segmentations. Theoretically, deep learning can learn without contouring the relevant information from images including cancer tumors. This drastically simplifies the data collection process and removes the human bias of contouring. However, it requires a large number of images before reaching such performance level, far larger than datasets currently used in research. To overcome this issue, some groups ${ }^{39}$ have used ImageNet repository ${ }^{40}$, which has a large number of non-medical images (e.g. landscape, animals, paintings, etc.), to train their networks. This study highlights a potential transferability of CNN and indicates a potential efficient way to build strong CNN, without requiring funds to acquire costly medical images. Both these new tools will likely widely change the radiomics field in the future.

\section{CONCLUSION}

In this thesis, we introduced the concept of radiomics, a rising field from quantitative imaging that aims to create a phenotypic atlas of cancer solid tumor. We have demonstrated its potential for important and critical steps of cancer management- diagnostic, treatment response, and evolution. As discussed above, several crucial steps must be carefully followed to ensure good practices and a bright future for radiomics. 


\section{REFERENCES}

1. The Moonshot Cancer Initiative. National Cancer Institute Available at: https://www.cancer.gov/research/ key-initiatives/moonshot-cancer-initiative.

2. The Precision Medicine Initiative. The White House Available at: https://obamawhitehouse.archives. gov/precision-medicine.

3. Frequently Asked Questions About Genetic and Genomic Science. National Cancer Institute Available at: https://www.genome.gov/19016904/.

4. Ow, T. J., Sandulache, V. C., Skinner, H. D. \& Myers, J. N. Integration of cancer genomics with treatment selection: From the genome to predictive biomarkers. Cancer 119, 3914-3928 (2013).

5. Bi, W. L., Zhang, M., Wu, W. W., Mei, Y. \& Dunn, I. F. Meningioma Genomics: Diagnostic, Prognostic, and Therapeutic Applications. Front. Surg. 3, 40 (2016).

6. Ebhardt, H. A., Root, A., Sander, C. \& Aebersold, R. Applications of targeted proteomics in systems biology and translational medicine. PROTEOMICS 15, 3193-3208 (2015).

7. Vaudel, M. et al. Exploring the potential of public proteomics data. PROTEOMICS 16, 214-225 (2016).

8. Tomescu, O. A., Mattanovich, D. \& Thallinger, G. G. Integrative omics analysis. A study based on Plasmodium falciparum mRNA and protein data. BMC Syst. Biol. 8, S4 (2014).

9. Tomescu, O. A., Mattanovich, D. \& Thallinger, G. G. Integrative Analysis of -Omics Data: A Method Comparison. Biomed. Eng. Biomed. Tech. (2013). doi:10.1515/bmt-2013-4311

10. Lambin, P. et al. Radiomics: Extracting more information from medical images using advanced feature analysis. Eur. J. Cancer 48, 441-446 (2012).

11. Aerts, H. J. W. L. The Potential of Radiomic-Based Phenotyping in Precision Medicine: A Review. JAMA Oncol. (2016). doi:10.1001/jamaoncol.2016.2631

12. Gillies, R. J., Kinahan, P. E. \& Hricak, H. Radiomics: Images Are More than Pictures, They Are Data. Radio/ogy 151169 (2015). doi:10.1148/radiol.2015151169

13. Coroller, T. P. et al. CT-based radiomic signature predicts distant metastasis in lung adenocarcinoma. Radiother. Oncol. 114, 345-350 (2015).

14. Fried, D. V. et al. Prognostic Value and Reproducibility of Pretreatment CT Texture Features in Stage III Non-Small Cell Lung Cancer. Int. J. Radiat. Oncol. (2014). doi:10.1016/j.ijrobp.2014.07.020

15. Huynh, E. et al. CT-based radiomic analysis of stereotactic body radiation therapy patients with lung cancer. Radiother. Oncol. 120, 258-266 (2016).

16. Huynh, E. et al. Associations of Radiomic Data Extracted from Static and Respiratory-Gated CT Scans with Disease Recurrence in Lung Cancer Patients Treated with SBRT. PloS One 12, e0169172 (2017).

17. Liu, Y. et al. Radiomic Features Are Associated With EGFR Mutation Status in Lung Adenocarcinomas. Clin. Lung Cancer 17, 441-448 (2016).

18. Yip, S. S. et al. Associations between somatic mutations and metabolic imaging phenotypes in non-small cell lung cancer. J. Nucl. Med. (2016). doi:10.2967/jnumed.116.181826

19. Coroller, T. P. et al. Radiomic phenotype features predict pathological response in non-small cell lung cancer. Radiother. Oncol. (2016). doi:10.1016/j.radonc.2016.04.004

20. Coroller, T. P. et al. Radiomic-Based Pathological Response Prediction from Primary Tumors and Lymph Nodes in NSCLC. J. Thorac. Oncol. 12, 467-476 (2017).

21. Aerts, H. J. W. L. et al. Defining a Radiomic Response Phenotype: A Pilot Study using targeted therapy in NSCLC. Sci. Rep. 6, 33860 (2016).

22. Kickingereder, P. et al. Large-scale Radiomic Profiling of Recurrent Glioblastoma Identifies an Imaging Predictor for Stratifying Anti-Angiogenic Treatment Response. Clin. Cancer Res. 22, 5765-5771 (2016).

23. Lu, L. et al. Robustness of Radiomic Features in [(11)C]Choline and [(18)F]FDG PET/CT Imaging of Nasopharyngeal Carcinoma: Impact of Segmentation and Discretization. Mol. Imaging Biol. MIB Off. Publ. Acad. Mol. Imaging 18, 935-945 (2016).

24. Parmar, C. et al. Robust Radiomics feature quantification using semiautomatic volumetric segmentation. Plos One 9, e102107 (2014). 
25. Kim, H. et al. Impact of Reconstruction Algorithms on CT Radiomic Features of Pulmonary Tumors: Analysis of Intra- and Inter-Reader Variability and Inter-Reconstruction Algorithm Variability. PloS One 11, e0164924 (2016).

26. Bogowicz, M. et al. Stability of radiomic features in CT perfusion maps. Phys. Med. Biol. 61, 8736-8749 (2016).

27. Zwanenburg, A., Leger, S., Vallières, M., Löck, S. \& others. Image biomarker standardisation initiativefeature definitions. ArXiv Prepr. ArXiv161207003 (2016).

28. Van Griethuysen, J. Computational Radiomics System to Decode the Radiographic Phenotype. Submitted

29. PyRadiomics. Available at: http://www.radiomics.io/.

30. Zhang, L. et al. ibex: An open infrastructure software platform to facilitate collaborative work in radiomics. Med. Phys. 42, 1341-1353 (2015).

31. Shafiq-UI-Hassan, M. et al. Intrinsic dependencies of CT radiomic features on voxel size and number of gray levels. Med. Phys. (2017). doi:10.1002/mp.12123

32. Quantitative Imaging Network | Informatics | Cancer Imaging Program (CIP). Available at: https://imaging.cancer.gov/informatics/qin.htm. (Accessed: 15th March 2017)

33. Limkin, E. J. et al. Promises and challenges for the implementation of computational medical imaging (radiomics) in oncology. Ann. Oncol. (2017). doi:10.1093/annonc/mdx034

34. Albain, K. S. et al. Radiotherapy plus chemotherapy with or without surgical resection for stage III nonsmall-cell lung cancer: a phase III randomised controlled trial. The Lancet 374, 379-386 (2009).

35. van Meerbeeck, J. P. et al. Randomized Controlled Trial of Resection Versus Radiotherapy After Induction Chemotherapy in Stage IIIA-N2 Non-Small-Cell Lung Cancer. JNCI J. Natl. Cancer Inst. 99, 442-450 (2007).

36. Cancer Treatments Description. National Cancer Institute Available at: https://www.cancer.gov/aboutcancer/treatment.

37. Marx, V. Biology: The big challenges of big data. Nature 498, 255-260 (2013).

38. LeCun, Y., Bengio, Y. \& Hinton, G. Deep learning. Nature 521, 436-444 (2015).

39. Antropova, N., Huynh, B. \& Giger, M. SU-D-207B-06: Predicting Breast Cancer Malignancy On DCE-MRI Data Using Pre-Trained Convolutional Neural Networks. Med. Phys. 43, 3349-3350 (2016).

40. ImageNet. Available at: http://www.image-net.org/. 
Summary 

Radiomics, an emerging field where many others intersect- medical physics, biostatistics, and computational biology, finds its significant application in recent cancer research. Radiomics can extract a large amount of data from medical images, uncovering advanced features that characterize tumors non-invasively through data analysis. These features can robustly create a unique phenotypic atlas for each tumor. Associating clinical information to this atlas has enabled the identification of new, reproducible, imagebased biomarkers, which have been used to predict tumor response to a specific treatment and understand tumor evolution or its intrinsic biology. Such tools will enable precision medicine in cancer treatment at an earlier time, providing crucial information to guide clinician decisions.

\section{PART 1: PREDICTION OF LUNG TUMOR RESPONSE TO CHEMORADIATION}

Currently, simple metrics such as tumor volume or diameter are used as surrogates for tumor response to treatment. These basics metrics were inadequate in predicting tumor response after chemoradiation. By using advances imaging features from pretreatment images, we could identify tumors that are likely responsive to chemoradiation. Those results can develop into a crucial tool for clinicians and potentially be used routinely to improve standard of care and outcomes. Better treatment decisions will help reduce physical, emotional, and financial burden on patients; and on a larger scale, it will reduce cost on the healthcare system through avoiding unnecessary surgeries, complications, and medications.

\section{PART 2: LUNG TUMOUR EVOLUTION AFTER RADIOTHERAPY TREATMENT}

The ability to characterize tumors that will likely develop distant metastasis or local recurrence based on their imaging traits at an early time point, especially prior to any treatment, is an advancement in cancer care. Once patients develop metastasis or experience recurrence, the disease become significantly harder to treat and the chance of survival quickly diminishes. This research findings will help clinicians make better decisions in patient management- particularly closer follow up and/or increase in chemotherapy for patients at risk to improve outcomes. This work in lung cancer can also be leveraged in understanding other types of tumors that frequently metastasize. 


\section{PART 3: TUMOUR INTRINSIC NATURE ASSESSMENT}

A cutting-edge method of extracting big data from positron-emission tomography (PET) and computed tomography (CT) images can provide additional information to quantify lung tumors caused by a genetic mutation. This information will help guide the most effective treatment by selecting the patient type that would most likely respond and experience good outcomes. This ability to differentiate will also help accelerate treatment for other patients that will not benefit from these mutation-specific medications. We also shown the potential of magnetic resonance images (MRI) to distinguish grade within meningioma patients. On a larger scale, this can contribute to reducing healthcare cost by improving diagnosis speed and accuracy, and as a result not paying for therapies that will not work for specific patients. This project also demonstrates the versatile application of radiomics in many different types of medical images, such as CT, PET, MRI, to better understand cancer. 


\section{Contribution Details}

Part 1: Prediction of lung tumor response to chemoradiation

Chapter 2: Wrote the manuscript, prepared all figures and did the statistical analysis.

Chapter 3: Wrote the manuscript, prepared figures 2-3 and did the statistical analysis.

Part 2: Lung tumor evolution after radiotherapy treatment

Chapter 4: Collected data, wrote the manuscript, prepared figure 1.

Chapter 5: Wrote the manuscript, prepared figures 2-3 and did the statistical analysis.

Chapter 6: Wrote the manuscript, prepared figure 2-4, did the statistical analysis.

Part 3: Tumor intrinsic nature ASSESSMENT

Chapter 7: Collected data, wrote the manuscript, prepared all figures and did all statistical analysis.

Chapter 8: Collected data, prepared and did the statistical analysis for figure 2, revised the manuscript.

Chapter 9: Did the analysis, prepared figures 2-3 and revised the manuscript 



\section{Acknowledgement Thank you}

Maman, papa merci pour tout, pour croire en moi, me pousser toujours plus loin (malgré mes notes catastrophiques au lycée) ainsi que de me donner le gout et des voyages et la curiosité pour d'autres cultures. Merci a ma famille pour tout le support et vivre ces aventures avec moi. Merci aux margoulins pour être toujours disponible, toujours présent dans ma vie malgré la distance et le temps qui passe. Vous êtes comme une deuxième famille (dysfonctionnelle) dont je suis fier de faire parti.

Cảm ơn vì tất cả mọi thứ Em, bây giờ chúng tôi chính thức là hai "doctors"! Tôi mong muốn cuộc phiêu lưu tiếp theo của chúng tôi.

I would like to thank my PhD advisors Dr. Hugo Aerts and Prof. Philippe Lambin for taking me on this adventure. I am glad I was part of the radiomics project while it was still growing (although it was already quite big when I joined, it has dramatically expanded after). To the assessment committee: Thank you Prof. Dirk de Ruysscher, Prof. Johan Bussink, Prof. Andrea Dekker, Prof. Peeters Ralf, Prof. W. Niessen for taking part in my assessment committee and for your time and effort reviewing this thesis.

Finally, I am thankful for all the amazing roommates, friends, colleagues, and researchers that I met during all these years in Boston. They gave me new insights, new cultures to learn from, as well as showed me what living and working in such an international environment means (every work lunch is like a UN meeting!).

What a journey. 



\section{Curriculum Vitae}

Thibaud Coroller was born in 1989 in Brest, France. He studied the scientific section at Kerneuzec High School in Quimperle from 2003 to 2007, where his passion for physics grew strong. After obtaining his baccalaureate with honours in 2007, he got his Licence in Physics from the University of South Brittany in 2010 and Master 1 from the University of Nantes in 2012. He completed his Master degree from the University Joseph Fourier in Grenoble in Medical Physics in 2013.

During his master program in 2013, Mr. Coroller did his internship at the Brigham and Women's Hospital and Dana Farber Cancer Institute, Boston, USA in the radiation oncology department. Under the supervision of Dr. Killoran, Mr. Coroller focused on lung cancer toxicities (ribs fracture and chest wall pain) post stereotactic radiation therapy. In the same department, he met his future supervisor Dr. Aerts. He joined Dr. Aert's team later in 2013 to start his joint PhD program between Dana Farber Cancer Institute and Maastricht University on radiomics.

He currently works as a postdoctoral researcher at the Computational Imaging and Bioinformatics Laboratory at the Dana Farber Cancer Institute and Harvard Medical School, Boston, USA under the supervision of Dr. Aerts.

\section{GRANTS AND AWARDS}

- Science Council Session Selection, American Association of Medicine in Physic, 2014 



\section{List of Publications}

1. Coroller TP*, Bi WL*, Huynh E et al. Radiographic Prediction of Meningioma Grade by Semantic and Radiomic features. PLoS ONE, 2017

2. Rios Velazquez E*, Parmar C*, Liu Y*, Coroller TP, Cruz Gisele, Stringfield O,Ye Z, Makrigiorgos M, Fennessy F, Mak R, Aerts HJWL. Somatic mutations drive distinct imaging phenotypes in lung cancer. Cancer Research, June 2017

3. Agrawal V, Coroller TP, Hou Y, Lee SW, Romano JL, Baldini EH, Chen AB, Kozono D, Swanson SJ, Aerts HJWL, Mak RH. Lymph node volume predicts survival but not nodal clearance in Stage IIIA-IIIB NSCLC. PLoS ONE , April 2017

4. Agrawal V, Coroller TP, Hou Y, et al. Radiologic-pathologic correlation of response to chemoradiation in resectable locally advanced NSCLC. Lung Cancer. 2016;102:1-8. doi:10.1016/j.lungcan.2016.10.002. Role: analysed the data, provided expert guidance and reviewed the manuscript

5. Huynh E, Coroller TP, Narayan V, et al. Associations of Radiomic Data Extracted from Static and Respiratory-Gated CT Scans with Disease Recurrence in Lung Cancer Patients Treated with SBRT. PLOS ONE. 2017;12(1):e0169172. doi:10.1371/journal.pone.0169172. Role: wrote the paper, provided expert guidance.

6. Coroller TP, Agrawal V, Huynh E, et al. Radiomic-based Pathological Response Prediction from Primary Tumors and Lymph Nodes in NSCLC. Journal of Thoracic Oncology. November 2016. doi:10.1016/j.jtho.2016.11.2226. Role: conceived of the project, analysed the data, prepared figures, wrote the paper.

7. Yip SS*, Kim J*, Coroller T, et al. Associations between somatic mutations and metabolic imaging phenotypes in non-small cell lung cancer. Journal of Nuclear Medicine. September 2016. doi:10.2967/jnumed.116.181826. Role: analysed the data, wrote the paper, provided expert guidance and reviewed the manuscript

8. Coroller TP, Agrawal V, Narayan V, et al. Radiomic phenotype features predict pathological response in non-small cell lung cancer. Radiotherapy and Oncology. April 2016. doi:10.1016/j.radonc.2016.04.004. Role: conceived of the project, analysed the data, prepared figures, wrote the paper. 
9. Huynh $E^{*}$, Coroller TP*, Narayan V, et al. CT-based radiomic analysis of stereotactic body radiation therapy patients with lung cancer. Radiotherapy and Oncology. June 2016. doi:10.1016/j.radonc.2016.05.024. Role: conceived of the project, analysed the data, prepared figures, wrote the paper, provided expert guidance.

10. Yip SSF, Coroller TP, Sanford NN, Mamon H, Aerts HJWL, Berbeco RI. Relationship between the Temporal Changes in Positron-Emission-Tomography-Imaging-Based Textural Features and Pathologic Response and Survival in Esophageal Cancer Patients. Frontiers in Oncology. 2016;6. doi:10.3389/fonc.2016.00072. Role: analysed the data, prepared figures, provided expert guidance and reviewed the manuscript

11. Yip SSF, Coroller TP, Sanford NN, et al. Use of registration-based contour propagation in texture analysis for esophageal cancer pathologic response prediction. Physics in Medicine and Biology. 2016;61(2):906-922. doi:10.1088/0031-9155/61/2/906. Role: analysed the data, prepared figures, provided expert guidance and reviewed the manuscript

12. Coroller TP*, Grossmann P*, Hou Y, et al. CT-based radiomic signature predicts distant metastasis in lung adenocarcinoma. Radiotherapy and Oncology. 2015;114(3):345350. doi:10.1016/j.radonc.2015.02.015. Role: conceived of the project, provided the data, wrote the paper, prepared figures, provided expert guidance and reviewed the manuscript

13. Coroller TP*, Mak RH*, Lewis JH, et al. Low Incidence of Chest Wall Pain with a RiskAdapted Lung Stereotactic Body Radiation Therapy Approach Using Three or Five Fractions Based on Chest Wall Dosimetry. PLOS ONE. 2014;9(4):e94859. doi:10.1371/journal.pone. 0094859. Role: conceived of the project, analysed the data, wrote the paper, prepared figures, provided expert guidance and reviewed the manuscript 


\section{Societal Impact and Valorizations}

In this thesis, we described the use of state-of-the-art advanced imaging features in combination with data science. The goal of this method is to investigate the association between image characteristics and tumor crucial information (relating to its nature, response, or evolution). This new method could be a potential tool for precision medicine. Any progress in precision medicine will benefit patients - such as avoiding surgery and tailoring treatment more effectively. In this context, several institutions have already started sharing tools to help. Published models can freely be found online. For example, MAASTRO clinic (http://www.predictcancer.org/) or the Memorial Sloan Kettering Cancer Center (https://www.mskcc.org/nomograms) have different models, from head and neck to rectum cancers, which are investigating overall survival or recurrence. However, these models have fairly simple metrics (such as patient age or tumor size). More advanced methods have emerged in the precision medicine toolbox to investigate relatively new field of research like genomics, proteomics, and radiomics. The major difference between these new methods and the more "conventional" one is the large number of parameters (from 1000 in radiomics to 20,000 in genomics). This distinction adds complexity to new tools and is prone to increased errors.

Several solutions can then be implemented to control those new techniques glitches. Open source initiative (https://opensource.org/) allows users, developers, and scientists to use and share technology at no cost while improving it. This ensure that researchers can widely share ideas and readers can replicate their results, at no cost nor legal issues. Our laboratory (http://www.cibl-harvard.org/) has been developing an open source code called PyRadiomics (http://www.radiomics.io/). The code allows extraction of radiomics features from medical images, which will help new teams investigate imaging analyses without the burden of starting from scratch, as well as allowing full flexibility. This project is done with the partnership of the Slicer development team (https://www.slicer.org/) which have extensive experience in developing open source software. Their product is a state-of-the-art software to visualize, segment, or quantify on any medical images (CT, PET, MRI) along with highly efficient open source libraries. While Slicer was mainly used for imaging management for this thesis, we also used open software such as $R$ (https://www.r-project.org/) or python (https://www.python.org/) that allow easy sharing across institutions and scientists. 
Open source is also valuable for information itself. Along with the performance and promise of new methods and software comes the curse of dimensionality. More complex methods will require larger sample size to train model with high accuracy and generality. Thus, several foundations have already helped increase data access such as the Cancer Imaging Archive (http://www.cancerimagingarchive.net/) with medical imaging, the Cancer Genome Atlas (https://cancergenome.nih.gov/) with genomics information. These platforms equipped scientists with direct access to highly curated medical information that can be immediately used to enhance science. 


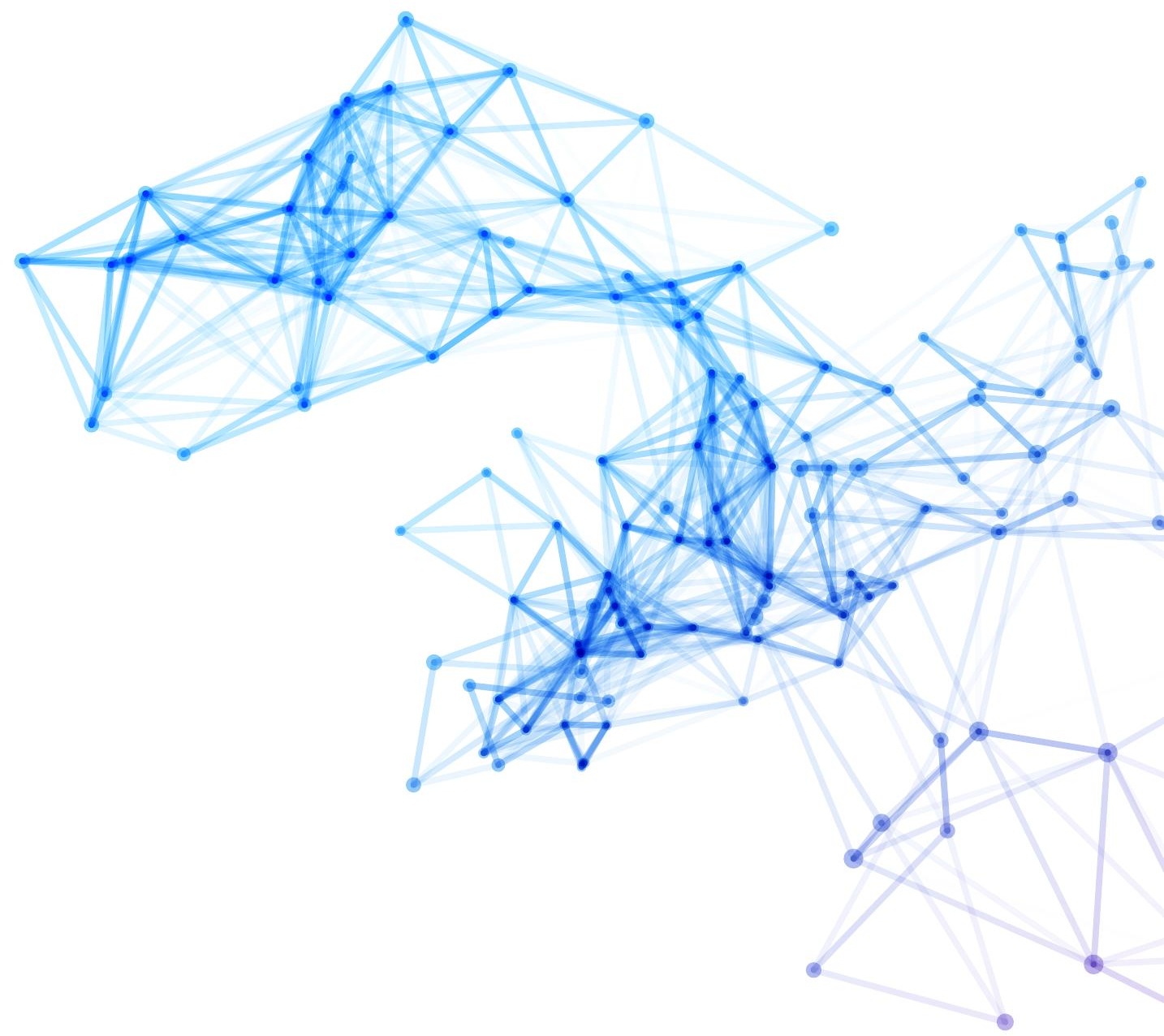

